\title{
RETRAN-02 INSTALLATION AND VERIFICATION FOR THE
}

CRAY COMPUTER

\section{PREPARED FOR \\ LOS ALAMOS NATIONAL LABORATORY}

PREPARED BY

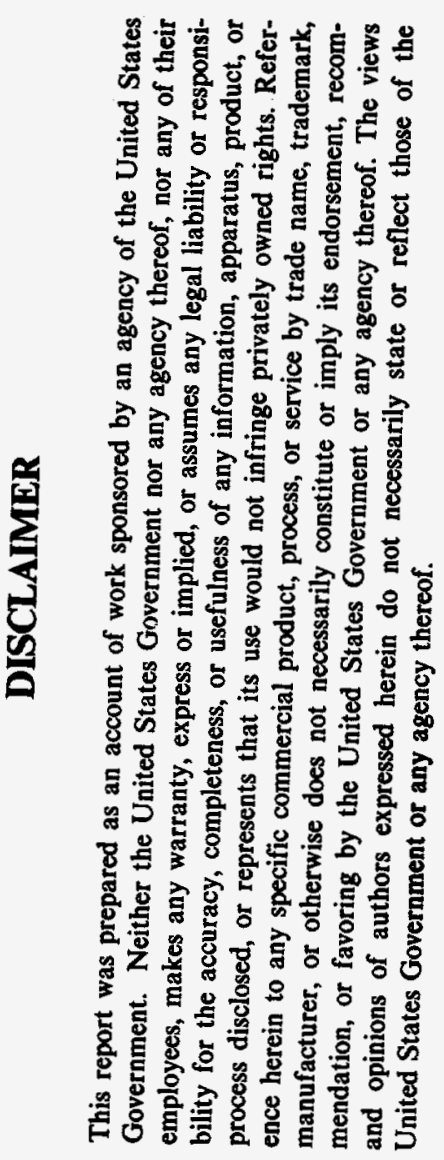

COMPUTER SIMULATION \& ANALYSIS, INC.

P. 0. BOX 51596

IDAHO FALLS, ID 83405

MARCH, 1990

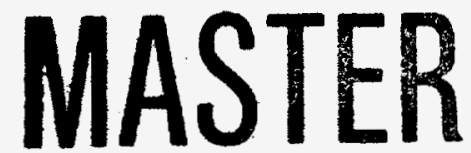

DISTRIBUTION OF THIS DOCUMENT IS UNLIMITED

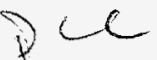




\section{DISCLAIMER}

Portions of this document may be illegible in electronic image products. Images are produced from the best available original document. 


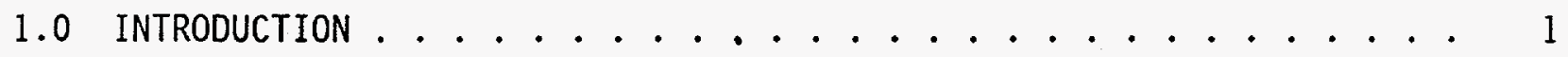

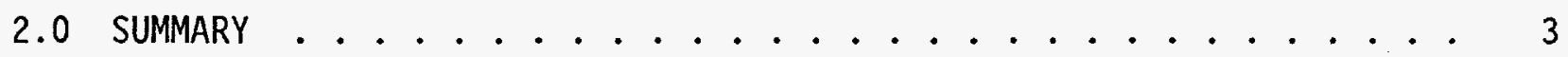

3.0 RETRAN-02 ENVIRONMENTAL LIBRARY CONVERSION .......... 5

4.0 RETRAN-02 CODE CONVERSION .................... 10

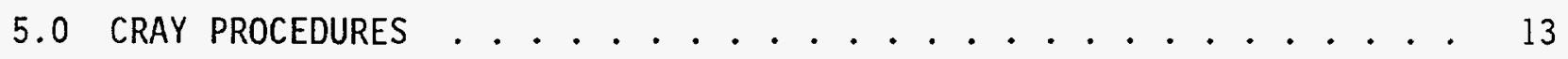

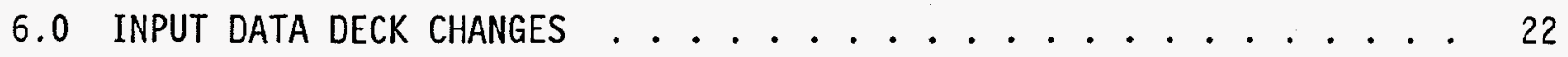

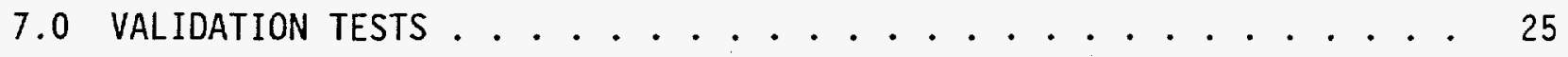

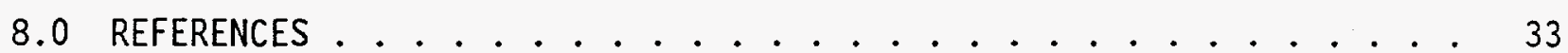

APPENDIX A - SAMPLE PROBLEM DATA OVERLAY PLOTS

APPENDIX B - ENVIRONMENTAL LIBRARY UPDATE DECK

APPENDIX $C$ - RETRAN-02 UPDATE DECK 


\subsection{INTRODUCTION}

The RETRAN-02 transient thermal-hydraulic analysis program[1] developed by the Electric Power Research Institute (EPRI) has been selected as a tool for use in assessing the operation and safety of the SP-100 space reactor system being developed at Los Alamos National Laboratory (LANL). The released versions of RETRAN-02 are not operational on CRAY computer systems which are the primary mainframes in use at LANL requiring that the code be converted to the CRAY system. This document describes the code conversion, installation, and validation of the RETRAN-02/MOD004 code on the LANL CRAY computer system.

The work was performed in accordance with CSA's Quality Assurance Program as implemented in the Project Requirements Document (PRD) LANL-PRD-001.[2] The PRD required that the work be based on a QA controlled version of the RETRAN02 code. CSA started the code conversion task using the CDC version of RETRAN-02/MOD004 provided by LANL for this work. LANL obtained the code from the Electric Power Software Center (EPSC) which maintained it under a quality assurance program. The code was received by LANL on a transmittal tape and unloaded to disk files by LANL. The CDC binary program libraries from the transmittal tape were converted by LANL to ASCII source files which were used to generate UPDATE[3] program libraries for the CRAY version. All revisions to the program source are maintained as correction identifiers in the new program library files stored in the LANL CFS file system. [4]

A11 work on code conversion and installation was done on the LANL CRAY-XMP computers using the CTSS operating system. [4] Installing RETRAN-02 on the LANL CRAY computing system required effort in three task areas, i.e.,

I. The RETRAN-02/MODO04 code includes a library of routines to extend FORTRAN capability and enhance portability among computer systems. This library required conversion to CRAY FORTRAN- 77 and involved converting the assembly language routines to FORTRAN-77 equivalent routines, modifying differences in syntax between CDC and CRAY FORTRAN-77 in the FORTRAN routines, and making the modifications 
required because of differences in the machine architecture and operating systems.

II. Converting the RETRAN-02/MOD004 program source to CRAY FORTRAN-77. This involved the elimination of coding associated with CDC specific user features that are not supported by CRAY FORTRAN-77 and making modifications that are required because of differences in the machine architecture and operating systems.

III. Installing and testing RETRAN-02/MODOO4 on the LANL CRAY-XMP computer. This task combined the results from Tasks I and II to produce an executable code. Program execution procedures were developed for installing the environmental library and the RETRAN02 code. Procedures were also developed for executing RETRAN-02 in each mode (initial run, restart, reedit, and plotting). The standard set of sample problems, restart cases, reedit cases, and plot cases were run and evaluated to verify that the code had been satisfactorily installed on the CRAY system.

A summary of the code installation and verification tasks is given in Section 2.0, the detailed changes to the environmental Tibrary and RETRAN-02 source are described in Sections 3.0 and 4.0 respectively, the CRAY procedures are described and listed in Section 5.0, changes to the RETRAN-02 input data are 1 isted in Section 6.0, and the validation cases with results are described in Section 7.0. Comparison plots of the CDC data from the transmittal tape and results from the CRAY code version are shown in Appendix $A$. The updates to the environmental library source are 1 isted in Appendix $B$ and the updates to the RETRAN-02 source are Tisted in Appendix $C$. 


\subsection{SUMMARY}

Several operations in the CDC version of RETRAN-02/MOD004 are carried out with the aid of assembly language coding either for efficiency or to provide user interface features not generally available through FORTRAN. Those functions which are supported by the CRAY FORTRAN-77 1 anguage were retained and those not supported were deleted. The assembly language routines were replaced with CRAY FORTRAN-77 versions. The user interface functions eliminated are listed below:

(1) dynamic field length adjustment at the time of job execution,

(2) monitoring the remaining CPU job time and terminating the job before the alloted CPU time is reached,

(3) requesting the restart tape dataset name and volume serial number through data supplied as part of a RETRAN-02 input dataset,

(4) displaying the restart tape dataset name and volume serial number in the program major edit output print file, and

(5) making each volume of a multiple volume restart dataset an independent dataset for restarting (this feature was not supported for IBM installations).

No models or numerical calculations in the RETRAN-02 program were modified or removed by the loss of the above functions and the CRAY installation is functionally equivalent to both the CDC and IBM versions with respect to its analysis capability. Input data for the code is unchanged from the description given in Volume 3 of the documentation[1] except for the deletion of the tape request cards. One additional difference in the CRAY code version which affects the input data is that the CRAY is sensitive to upper and lower case characters. All character data such as minor edit request variables should be in lower case form. 
Procedures were developed to execute the RETRAN-02 code under the CTSS system at LANL using the CFS file system. Input and restart datasets are assumed to reside in the CFS system and must be retrieved into the users local file space prior to program execution. Responsibility for the disposition of newly created files belongs to the user. Procedures were also developed for maintenance of both the environmental library and the RETRAN-02 code which include updating, compiling, building a library as necessary, and loading the revised program.

Installation of RETRAN-02 on the LANL CRAY system was verified by running the sample problems (ten initial cases, two restart cases, two reedit cases, and two plot cases) from Volume 3 of the RETRAN-02 documentation. [1] The results from the ten initial cases from the CRAY code version were compared to available results from corresponding cases obtained from the EPSC transmittal tape. Graphical comparisons of minor edit variables were made where sufficient data was available, otherwise visual comparisons of major edit values were made. Comparisons were also made of the number of time steps required for each version. In all cases any differences between the CRAY version and $C D C$ version results were within acceptable bounds except for the TTWOB point kinetics case. The maximum time-step size specified in the data deck from the EPSC transmittal tape exceeded the range where the solution is converged. Rerunning the case with smaller time steps resulted in a converged solution where the CRAY version results were within the acceptable tolerance range of the $C D C$ results. 


\subsection{RETRAN-02 ENVIRONMENTAL LIBRARY CONVERSION}

The RETRAN-02 environmental 1ibrary contains routines or sub-code packages for handling dynamic memory allocation, input data processing, plotting, bit manipulation, $1 / 0$ processing, and other miscellaneous functions. Many of these library routines are written in FORTRAN and only discrepancies with CRAY FORTRAN-77 needed correcting. A number of routines (approximately 25) are written in CDC assembler code and all of these routines required replacement with functionally equivalent FORTRAN-77 routines where possible. In some instances it was not possible to duplicate the functionality of the CDC routines with CRAY FORTRAN- 77 and those routines were dummied or eliminated. Specific modifications to the various environmental routine categories are summarized in the following sections. The source and deck names for the environmental library are in lower case character form.

\subsection{FTB Package}

The FTB storage allocation package is used in RETRAN-02 to dynamically assign main memory for FORTRAN arrays at execution time. There is also a feature that allows the field length (main memory size) to be adjusted to free unused memory. This feature cannot be readily implemented on the CRAY using only FORTRAN-77 and was not converted. On the CRAY, FTB will allocate storage from a large fixed length array of 130000 words. The subroutine FTBSET was modified to pass this length to the FTB initialization routines eliminating the need for subroutine GETCOR. The COMDECK OPSY\$CA and the assembly language subroutines GETCOR, FRECOR, and REDUCE which performed the dynamic memory allocation operation were deleted. The file descriptions for the FTB package are kept in four (4) word units in the FTB storage array. Information in these descriptions is packed into 32-bit half words. An assembly language routine named IA performs the packing and unpacking operation in the CDC library. This routine was deleted in the CRAY version and boolean (either RETRAN-02 library or CRAY intrinsic) functions are used to pack and unpack the file description words. 
The CDC version of FTB also supports special purpose disk files referred to as process files. Process files are not needed and rely on a rather complicated I/0 procedure that is system dependent and must be written in assembly language. The use of process disk files in the CRAY RETRAN-02 version was replaced with standard FORTRAN-77 files. Thus, subroutines FTBIO, PROCES, PROC4, OPEN, CLOSE, TRNCAT, CPYFIL, GET, ISFOPEN, ISFPRC, MXSETS, NFSET, and NFUNIT were eliminated from the CRAY version of the environmental library.

\subsection{INP Free Form Input Processing Package}

The INP free form input processing package is implemented for the CDC version by using a combination of FORTRAN and assembly language subroutines. For the CRAY implementation, the assembly language routines were replaced with FORTRAN routines that are functionally equivalent. The assembly language routines that were replaced with FORTRAN-77 versions were CVI, LINK, and PCKUPK. The INP processing package stores the input data in a table in such a manner that data from a particular card can be located readily. The key subroutines for building, finding data locations, and maintaining the data table are INP, LINK, INP8, and INP10. All of the INP subroutines were deleted from the CDC library source and replaced with the new versions compatible with the new INP, CVI, LINK subroutines. The subroutines in this group include INP, CVI, LINK, PCKUPK, INP2, INP4, INP5, INP6, INP7, INP8, INP10, and MODER. A new COMDECK INPC was added to the environmental library source.

\subsection{PLOTMC Plotting Package}

The PLOTMC plotting package uses CalComp compatible subroutine calls to generate $X-Y$ plots. The CDC version of PLOTMC provides an assembly language version of the subroutine SYMBOL which does character string plotting. Since SYMBOL is a standard Calcomp plotting subroutine, it can be obtained from the Calcomp plot library that is required to satisfy references to the other Calcomp (or compatible) subroutines PLOT and PLOTS. The assembly language version of SYMBOL was deleted from the CRAY environmental library. 


\subsection{Bit Manipulation Routines}

Several of the CDC assembly language routines perform bit manipulation functions, are short single purpose functions, or access intrinsic functions whose names differ between system vendors. The bit manipulation and intrinsic function routines are present in the environmental library to maintain maximum transportability of the RETRAN-02 program among various vendor systems. These assembly language routines were replaced with FORTRAN equivalents using CRAY intrinsic FORTRAN-77 functions. Routines in this group include MASKF, DSL, DSR, DOR, DAND, IAND, IOR, ISL, ISR, COMPAR, PACK and UNPACK. Both the integer and real shift functions and the boolean functions use the full 64-bit word length of the CRAY. Bit packing also uses the 64-bit word length and 8-bit character length for the CRAY system.

\subsection{Buffer Input/Output}

The buffered input/output routines for the CDC version are written in assembly language and provide features that allow the user to request data sets via input data (rather than conventional job control language) and end-of-volume (EOV) processing that allows each volume of a multi-volume set to be used independently for restart problems. Entry point BUFREQ is used to request tape data sets dynamically at execution time. This entry point was eliminated from subroutine BUFOUT. Subroutine $\mathrm{C} I O=$ is a $\mathrm{CDC}$ system routine that is used by the assembly language version of BUFOUT. CIO= was eliminated from the CRAY environmental library. The other routines (BUFOUT, BUFIN, BUFWEF, and BUFSKP) were replaced with FORTRAN-77 versions which were functionaliy equivalent to their assembly language counterparts. Record structures and formats are consistent with the FORTRAN-77 binary input/output requirements.

Tape datasets are not available on the CRAY system but can be used for file archiving. Datasets such as RETRAN-02 restart files are initially written to files in the CRAY user's local area and may then be saved or deleted. References to tape datasets were removed from the RETRAN-02 code and the corresponding data cards removed from the data decks. 


\subsection{Extended I/0 Routines}

Several extended I/ 0 subroutines are used by RETRAN-02 to write information to the job $\log$ and to perform incore reads and writes. The incore read/write routines are written using CDC ENCODE and DECODE extensions to FORTRAN. These routines will be used for the CRAY versions using the CRAY ENCODE and DECODE extensions. CRAY FORTRAN supports the REMARK routine (CDC FORTRAN 1ibrary) that allows messages to be written to the job log. No changes were necessary to use these routines.

\subsection{System Interrogation Routines}

The system interrogation routines provide facilities to interrogate the operating system for elapsed CPU and wall clock times, remaining CPU time in excess of the job time limit, date and interval timing. Several of these functions are not available with CRAY FORTRAN. In particular, the NOTIM and ICPSEC functions that are used to terminate RETRAN-02 prior to exceeding the job time limit are not available. The NOTIM function was replaced with a dummy version to satisfy external references in the code and the function ICPSEC was deleted from the library. References to IPCSEC in the RETRAN-02 source were deleted. The other timing and date functions are available in CRAY FORTRAN-77.

The subroutine timing function TIMSET with entry points TIMEL and TIMER was replaced with a FORTRAN-77 version.

\subsection{Miscellaneous Routines}

The RETRAN-02 environmental library contains a few miscellaneous routines written in assembly language which were rewritten using CRAY FORTRAN-77 or deleted. These routines include LOCF, COMPAR, ERRMON, FINDEP, FLOATR, LCMBUF, LCMDUM, MOVE, MOVEI, and ZEROUT. CRAY FORTRAN supports a LOCF function in which the equivalent operation to the CDC LOCF has the entry point LOCX. References to LOCF were replaced with references to LOCX in the RETRAN-02 
code. Subroutine FINDEP is used in the CDC version to locate the address of a primary entry point which is used to define an FTB file that coincides with the routine location in memory. This feature is not used in the CRAY version and the subroutine FINDEP was eliminated. The CRAY system does not have extended core and the routines LCMBUF and LCMDUM for accessing LCM were el iminated.

The subroutines COMPAR, FLOATR, MOVE, MOVEI, and ZEROUT written in CDC assembly language and were replaced with CRAY FORTRAN-77 equivalent routines.

Subroutine FORTER, which defines the CDC error recovery process, was made into a dummy routine. The ERRMON subroutine, called during error processing in the CDC code version, is no longer needed and was deleted from the library. The FABEND routine, called from RETRAN-02 when errors are detected, was revised to call the CRAY traceback routine. References to the CDC function, STRACE, which processes the traceback were satisfied with a library routine which calls the CRAY traceback routine. 


\subsection{RETRAN-02 CODE CONVERSION}

The RETRAN-02 MOD004 source code was modified for the CDC FORTRAN-77 compiler. Programming changes were required to convert the source to accommodate the different word length of the CRAY system, revise the logic associated with disabled, deleted, or revised program features in the CRAY version, and to eliminate any syntax differences between the CDC and CRAY compilers. The source and deck names for the RETRAN-02 program are in lower case character form.

Main memory storage for the CDC version resides between the beginning of the FTB common block and the end of the assigned region or field length. The amount of available storage can then be adjusted by changing the field length at execution time. Once the storage requirements are determined, unused space is freed by reducing the region size. This feature was deleted for the CRAY version. A large fixed dimension array of 130000 words is used for the storage pool from which FTB files are obtained. The array dimension is defined once in the subroutine INPUT and can be revised if more space is required.

The CDC version of RETRAN-02 stores data over unused coding blocks to minimize unused'memory. This is necessary since RETRAN-02 does not use extended memory and the size of main memory is relatively small. Subroutine RESOPT is used to map FTB files onto optional program blocks. FTB files corresponding to unused options are deleted, thus, freeing the space for later use by new FTB files. The segmented loader is used to overlay the input segments and to force the transient segments to be loaded in a particular order that corresponds to the order that FTB files are reserved in RESOPT. This feature was deleted in the CRAY version and the subroutine RESOPT revised to remove calls to the function FINDEP and to save FTB files.

The FTB package also supports special purpose disk files referred to as process files. Process files are used with minor edits and printer plots. 
The process file routines were not implemented for the CRAY version and the routines that used process files modified to use standard FORTRAN files.

Several routines in RETRAN-02 make use of information packed into half words or characters packed into real words. Word and character lengths used for packing and unpacking are controlled by a logical variable, IBMRUN. The code as received from EPSC is set to use 60-bit word lengths and six-bit character lengths for the non-IBM applications. The non-IBM option was retained and the respective bit lengths increased for the CRAY version. Half-word lengths were set to 32 bits and character lengths to eight bits.

Internal tape volume requests processed through the BUFREQ entry in the BUFOUT routine were eliminated since the BUFREQ entry was deleted. Coding associated with character field processing and checking for tape data set requests were deleted from the RETRAN-02 program. Tape EOV processing was also disabled making a multi-volume restart file a contiguous data set. Coding associated with handling multiple volume data sets with EOV processing was deleted since it is an unused feature.

The remaining source modifications for RETRAN-02 included removing the external file definitions from the PROGRAM card and opening each file with an explicit OPEN statement, deleting references to unit 81 which was the DATATRAN history file, deleting or replacing references to the routine PDUMP with FORTRAN write statements since the CRAY does not support PDUMP, and adding dummy routines to satisfy external references in the plotting routines. The page title was changed from specifying an EPSC code version to a LANL version.

External units used by the CRAY version of RETRAN-02 reference local files in either a read or write mode but not in a read/write mode. Existing local files which will become output files must be deleted prior to executing RETRAN-02. The procedures discussed in Section 5.0 contain the appropriate file deletions. External units referenced by RETRAN-02 (excluding scratch units) are 7 isted in Table 1 . 
TABLE 1

RETRAN-02 EXTERNAL FORTRAN FILE DESCRIPTION

\begin{tabular}{|c|c|c|}
\hline $\begin{array}{l}\text { Program } \\
\text { Module }\end{array}$ & $\begin{array}{l}\text { File } \\
\text { Name }\end{array}$ & Description \\
\hline RETRAN & $\begin{array}{l}\text { tape5 } \\
\text { tape6 } \\
\text { tape12 } \\
\text { tape14 } \\
\text { tape40 }\end{array}$ & $\begin{array}{l}\text { input data file (READ only) } \\
\text { output print file (WRITE only) } \\
\text { boundary condition data file if running a boundary } \\
\text { condition case (READ only) } \\
\text { new restart dataset if requested by input data } \\
\text { (WRITE only) } \\
\text { one-dimensional cross-section dataset if running a } \\
\text { one-dimensional kinetics case (READ only) }\end{array}$ \\
\hline RESTRT & $\begin{array}{l}\text { tape5 } \\
\text { tape6 } \\
\text { tape12 } \\
\text { tape13 } \\
\text { tape14 } \\
\text { tape40 }\end{array}$ & $\begin{array}{l}\text { input data file (READ only) } \\
\text { output print file (WRITE only) } \\
\text { boundary condition data file if running a boundary } \\
\text { condition case (READ only) } \\
\text { old restart dataset (READ only) } \\
\text { new restart dataset (WRITE only) } \\
\text { one-dimensional cross-section dataset if running a } \\
\text { one-dimensional kinetics case (READ only) }\end{array}$ \\
\hline REEDIT & $\begin{array}{l}\text { tape5 } \\
\text { tape6 } \\
\text { tape13 } \\
\text { tape60 }\end{array}$ & $\begin{array}{l}\text { input data file (READ only) } \\
\text { output print file (WRITE only) } \\
\text { old restart dataset (READ only) } \\
\text { special edit dataset (WRITE only) }\end{array}$ \\
\hline PLOTER & $\begin{array}{l}\text { tape5 } \\
\text { tape6 } \\
\text { tape11 } \\
\text { tape12 } \\
\text { tape13 }\end{array}$ & $\begin{array}{l}\text { input data file (READ only) } \\
\text { output print file (WRITE only) } \\
\text { plot dataset number } 1 \text { (READ only) } \\
\text { plot dataset number } 2 \text { if requested by input data } \\
\text { (READ only) } \\
\text { plot dataset number } 3 \text { if requested by input data } \\
\text { (READ only) } \\
\text { plot dataset number } 4 \text { if requested by input data } \\
\text { (READ only) }\end{array}$ \\
\hline
\end{tabular}




\subsection{CRAY PROCEDURES}

The modified RETRAN-02 source code and environmental library were installed on the CRAY computer using the CRAY UPDATE program[3] for source maintenance, the CFT FORTRAN-77 compiler, [5] and the LDR loader. [4] A normal program load (not segmented) was used to install the code. Procedures for the XEQ program[4] were developed to maintain the RETRAN-02 program and library and to execute RETRAN-02 in its various modes. These procedures are listed and described in this section. Existing files are assumed to reside in the CFS file system and any local files with the same name are destroyed. Requesting non-existent files from CFS results in a message to the user but does not cause an error. Newly created files are normally left in the local file area without saving them. It is the users responsibility to properly save or dispose of any new files.

\subsection{Environmental Library Maintenance Procedure}

A procedure for the XEQ program is listed in Table 2 for updating, compiling, and building an environmental program library. The library will consist only of those routines compiled in the current procedure execution since a previously existing compiler 'lgo' file is not merged. The old UPDATE program Tibrary is assumed to be named 'nenvpl' and a new program library named 'envp10la' will be created. The UPDATE directives are in a local file named 'upd01.ev' and the UPDATE output listing is named 'envlist'. The compiler output is limited to error messages only. The final library is a file named ' $r 2 m 41 i b '$. In this example, the newpl and library are saved in the CFS system. The leading character ' $r$ ' in each instruction is a sentinel for the XEQ program. This procedure is executed by the statement 'xeq enviproc' where 'enviproc' is the file name of the procedure. 
TABLE 2

PROCEDURE ENVIPROC

PROCEDURE TO UPDATE, COMPILE, AND BUILD A LIBRARY

rdestroy envp101a

rdestroy nenvpl

rdestroy r2m4lib

rcfs get nenvp 1

rupdate $p=$ nenvp $1, c=$ compile, $n=$ envp $101 a, 1=e n v 1$ ist $, i=u p d 01 . e v, c d$, in,$i d$ rcft $b=$ envigo, $i=$ compile, $1=0$

rbuild $b=$ envigo, $n 7=r 2 m 47$ ib, $a, 7=1$ iblist, $70=m$

rcfs store envpiola

rcfs store $\mathrm{r} 2 \mathrm{~m} 4 \mathrm{ib}$

rend 


\subsection{RETRAN-02 Program Maintenance Procedure}

A procedure for the XEQ program is listed in Table 3 for updating, compiling, and loading the RETRAN-02 program. The old UPDATE program library is assumed to be named 'newp101a' and a new program library named 'newpl01b' will be created. The UPDATE directives are in a local file named 'r2upd05' and the UPDATE output listing is named 'r2updlst'. The compiler output is 1 imited to error messages only. An old 1go file (compiled source file) named 'retlgo.b' will be merged with the newly created lgo file from the compiled source and the result saved in a file named 'retlgo.c'. Libraries made available to the loader include the RETRAN-02 environmental 1ibrary named ' $\mathrm{rm} 41 \mathrm{ib}$ ' and the cft system Tibraries. The leading characters ' $r$ ' and ' $c$ ' in the instructions are sentinels for the XEQ program. This procedure is executed by the statement 'xeq ret2ucl' where 'ret2ucl' is the file name of the procedure. Newly created files are saved in the CFS system in this example.

\subsection{RETRAN-02 Program Execution Procedures}

The procedure 'exretran' for the XEQ program listed in Table 4 will execute the RETRAN-02 program. The leading character ' $r$ ' in the instructions is a sentinel for the XEQ program. This procedure uses a substitutable name which allows the user to run different cases without retyping the procedure each time. The substitutable name is designated by the characters '\%m' and the XEQ name is substituted when XEQ is called. The local copy of the restart file is switched to a 'read-write' mode by the command 'switch \&merst rw.' and the local file overwritten. File naming conventions in these procedures use a deck name of one to five characters indicated by '*****' with a postscript identifier as follows:

$\star * * * *$ dek $=$ input dataset for initial case (complete deck)

$* * * * * x S C=$ one-dimensional cross-section file

$* * * * * r d k=$ input dataset for restart case

$* * * * *$ red $=$ input dataset for reedit case 
TABLE 3

PROCEDURE RET2UCL

PROCEDURE TO UPDATE, COMPILE, AND LOAD RETRAN-02

rdestroy newp101b

rdestroy retlgo.b

rdestroy retlgo.c

rdestroy newp101a

rcfs get newpl01a

rcfs get retigo.b

rupdate $p=$ newp $101 a, c=$ compi 1 e, $1=r 2$ upd $7 s t, i=r 2$ upd05,

$c \& n=$ newp $101 b, c d$, in $, i d, e d$

rcft $b=$ newlgo, $i=$ compile, $1=0$

rdestroy oldlgo

rcopy retlgo.b oldlgo

rbuild $b=$ newlgo, $01=01 \mathrm{~d} l g 0, r, 1=1$ iblist, $10=m$

$r l d r \quad i=01 d l g o, 1 i b=(r 2 m 41 i b, c f t m a t h, c f t l i b), c s=170140 b, x=$ retran, $m l=m a p$,

$\mathrm{cmo}=\mathrm{su}$

rcfs store retran

rcopy oldigo retigo.c

rcfs store retigo.c

rcfs store newp101b

rend 
TABLE 4

\title{
PROCEDURE EXRETRAN
}

RETRAN PROCEDURE TO EXECUTE AN INITIAL CASE

\author{
rdestroy tape5 \\ rdestroy tape14 \\ rdestroy tape 40 \\ rdestroy \%medek \\ rdestroy \%mexsc \\ rcfs get \%mexsc \\ rcopy \%mexsc tape 40 \\ rcfs get \%medek \\ rcopy \%medek tape5 \\ rretran \\ rswitch \%merst rw. \\ rcopy tape14 \%merst \\ rend
}




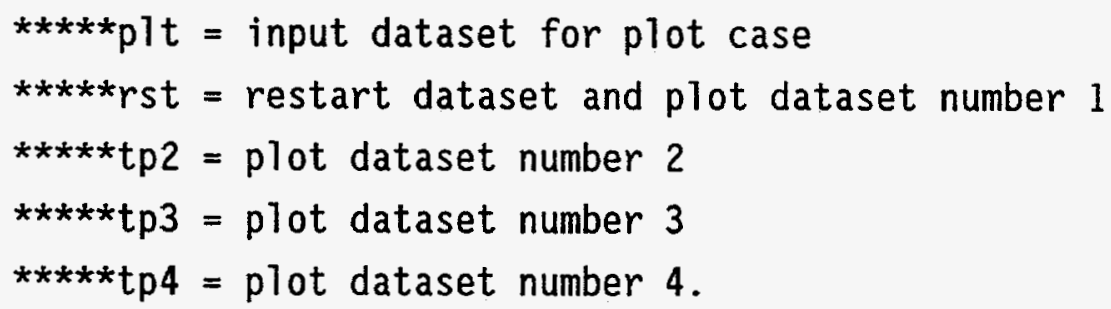

The procedure is executed by copying the procedure to a local file having the problem name and then calling the XEQ program with that name as illustrated in the following commands:

copy exretran ttwob xeq ttwob

assuming the procedure is named 'exretran' and one is running the 'ttwob' sample problem. Newly created files are not saved in the CFS system in this example.

A procedure to execute a restart case is 1 isted in Table 5 . This procedure differs from the procedure in Table 4 by copying a restart file to the local file 'tape13' and using a different name '\%merdk' for the input data file to avoid conflicts with the initial case data deck file name.

A procedure to execute a reedit case is Tisted in Table 6 . The input data file is named '\%mered'.

A procedure to execute a plot case is 1isted in Table 7 . This procedure allows up to four restart/plot data files to be defined for use in overlay and/or combination plots. The primary data file is assumed to be named '\%merst' and copied to the local file 'tapell'. The input data file is named '\%mep1t'. Output from the plot module is limited to the print file 'tape6' as no plotter specific routines are currently included in the RETRAN-02 program. 


\title{
TABLE 5
}

PROCEDURE EXRETRST

\section{RETRAN PROCEDURE TO EXECUTE A RESTART CASE}

\author{
rdestroy tape5 \\ rdestroy tape14 \\ rdestroy tape 40 \\ rdestroy tape13 \\ rdestroy \%merdk \\ rcfs get \%merst \\ rcopy \%merst tape13 \\ rdestroy \%mexsc \\ rcfs get \%mexsc \\ rcopy \%mexsc tape 40 \\ rcfs get \%merdk \\ rcopy \%merdk tape5 \\ rretran \\ rswitch \%merst rw. \\ rcopy tape14 \%merst \\ rend
}


TABLE 6

PROCEDURE EXREEDIT

RETRAN PROCEDURE TO EXECUTE A REEDIT CASE

rdestroy tape5

rdestroy tape13

rdestroy tape60

rdestroy \%mered

rdestroy \%merst

rcfs get \%merst

rcopy \%merst tape 13

rcfs get \%mered

rcopy \%mered tape 5

rretran

rend 
TABLE 7

PROCEDURE EXRETPLT

RETRAN PROCEDURE TO EXECUTE A PLOT CASE

\author{
rdestroy tape5 \\ rdestroy tapel1 \\ rdestroy tape 12 \\ rdestroy tape13 \\ rdestroy tape14 \\ rdestroy \%merst \\ rcfs get \%merst \\ rcfs get \%metp2 \\ rcfs get \%mept3 \\ rcfs get \%mept4 \\ rcopy \%merst tapel1 \\ rcopy \%metp2 tape12 \\ rcopy \%metp3 tape13 \\ rcopy \%metp4 tape14 \\ rdestroy \%meplt \\ rcfs get \%meplt \\ rcopy \%meplt tape5 \\ rretran \\ rend
}




\subsection{INPUT DATA DECK CHANGES}

Minor changes in the input data decks as described in Volume 3 of the RETRAN02 documentation[1] were required for the CRAY version of the code. These changes were limited to the deletion of the tape request cards for the restart, reedit, boundary condition, and plot files. In addition, since the CRAY computer software is character case sensitive, character data such as minor edit variables and control block types must be in lower case characters. Input data changes from Volume 3 are as follows.

Section IV.4.0 Boundary Condition Tape Request Data Cards $01 \times \times 20$ and $01 \times \times 2 Y$. Delete these cards from data decks where $X X$ ranges from 1 to 99 and $Y$ ranges from 1 to 9 .

Section IV.5.0 Data Tape Label Data Cards $01 \times X 40$ and $01 \times X 4 Y$.

Delete these restart tape request cards from data decks where $X X$ ranges from 1 to 99 and $Y$ from 1 to 9 .

Section V.3.0 Data Tape Request Cards 010130 and 01013 Y

Delete these restart tape request cards from data decks where $Y$ ranges from 1 to 9 .

Section V.4.0 Data Tape Label Data Cards $01 \times \times 40$ and $01 \times \times 4 Y$ Delete these restart tape request cards from data decks where $X X$ ranges from 1 to 99 and $Y$ from 1 to 9.

Section VI.3.0 Data Tape Request Data Cards $01 \times \times 30$ and $01 X \times 3 Y$ Delete these reedit tape request cards from data decks where $X X$ ranges from 1 to 99 and $Y$ from 1 to 9 .

Section VII.3.0 Data Tape Request Data Cards 01XXYO and 01XXYS Delete these plot tape request cards from data decks where $X X$ ranges from 1 to $99, Y$ ranges from 1 to 9 , and $S$ from 1 to 9 . 
Section IV.6.0 Minor Edit Variable Data Cards 02000Y

The alphabetic characters in the symbol lists in Tables IV.6-1 through IV.6-17 are input to RETRAN-02 as lower case characters.

Section IV.17.4 Decay Heat Power History Interval Data 14700X

The units parameter in word 3 is in lower case characters.

Section IV.25.4 Control Input Definition Data Cards 702XXX

The variable symbol 'CSYM' in word 2 is input with lower case characters.

Section IV.25.5 Control Input Description Data Cards 703XXX

The variable symbol 'ITYPE' in word 2 is input with lower case characters.

Section VI.5.0 Auxiliary Data File Format Description Card 03000Y

The character string 'binary', if used, is in lower case characters.

Section VII.4.0 Independent Axis Specification Data Cards 02XX0Y

The character variables in words $1,3,7,8,9$, and 10 are input as 10 wer case characters.

Section VII.5.0 Dependent Axis Specification Data Cards $03 \times X Y Z$

The character variables in words $1,5,6,7$, and 8 are input as lower case characters.

Section VII.6.0 Plot Curve Request Data Cards 4 XXYZS

The character variable in word 1 is input as lower case characters.

Section VII.7.2 Parametric Plot Curve Request Data Cards 50XXYS

The character variables in words $5,8,11$, etc., are input as lower case characters.

Section VII.7.3 Parametric Plot Curve Request Data Cards 51XXYS

The character variable in word 5 is input as lower case characters. 
Section VII.8.0 Relap3, Relap4/002/003/005/007 Plot Request Symbols This option is not available in the CRAY version.

Section VII.9.0 Retran Stranger Data Tape Format and Structure

This option is not available in the CRAY version. 


\subsection{VALIDATION TESTS}

A variety of test cases were run on the CRAY version of RETRAN-02 to ensure that the program was correctly converted and installed. Test cases were run for the RETRAN program, the restart, reedit, and plotting options. The ten sample problems on the EPSC transmittal tape were run as validation cases and the results were compared against the corresponding CDC cases included with the EPSC transmittal. The restart, reedit, and two plot test cases given in the RETRAN-02 User's Manual[1] were run as validation cases for these options. The validation cases are discussed in more detail below.

\subsection{RETRAN}

The ten sample problems that were included with the RETRAN-02 transmittal package from the EPSC were run to test the CRAY version of RETRAN-02 MOD004. These problems are as follows:
(1) Standard Problem One,
(2) Standard Problem Five,
(3) Eight Volume Sample Problem,
(4) Standard Problem Four,
(5) Uncontrolled Rod Withdrawa1,
(6) Turbine Trip Without Bypass (Point Kinetics),
(7) Two-Dimensional Flow Field,
(8) Secondary-Side Sample Problem,
(9) Turbine Trip Without Bypass (One-Dimensional Kinetics), and
(10) Anticipated Transient Without Scram.

The results from these cases were checked against the respective sample problem output files from the transmittal tape by comparing the number of time steps taken and by making overlay plots for several of the minor edit variables from the CDC and CRAY version. The sample problem output from the transmittal tape did not contain minor edits for the following cases: Standard Problem One, Standard Problem Four, and the Two-Dimensional Flow Field. Point 
values from the major edits in these latter cases were included on the plots without connecting curves except for the Two-Dimensional Flow Field which was not plotted. The latter case converges to a steady-state condition and values in the final edit were compared as a check on the correctness of the solution.

The time-step comparisons between the CDC and CRAY cases are listed in Table 8 for the ten sample problems. In seven cases, the actual number of time steps taken by the CRAY version were the same as for the respective CDC cases. In two cases, i.e., the Secondary-Side Problem and the TTWOB, the number of actual time steps were within two of the CDC cases. The CRAY calculation of the ATWS problem took eight (8) fewer time steps than did the CDC calculation. The CPU times for both the CRAY and CDC versions are also listed in Table 8 for each case.

Some differences were expected in the calculated results since the CRAY and CDC word lengths and floating point hardware are different. The FORTRAN library functions, e.g., SQRT, EXP, ALOG, etc., could also generate slightly different results between the two systems. Three categories of differences between the CRAY and CDC results were identified[6] and a corresponding measure of acceptability for these differences defined. The categories are:

(1) timing, time-of-day, data, or operating system dependent results, e.g., CPU time, memory size, etc.;

(2) differences of similar magnitude numbers that are approximately equal to zero, within the accuracy of the given machine's representation of floating point words with a fixed number of bits; and

(3) the other calculated quantities. 
TABLE 8

TIME-STEP COMPARISON BETWEEN THE CDC AND CRAY SYSTEM

FOR RETRAN-02 SAMPLE PROBLEMS

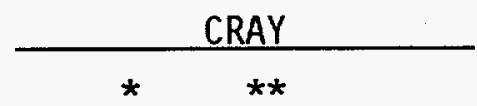

Problem Problem end ATS STS CPU time $\mathrm{sec}$

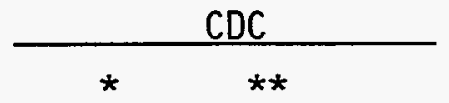

ATS STS CPU time sec

Std Prb1

0.465

223

45

3.8

$223 \quad 45$

20.8

Std Prb5

5.0

485

345

10.1

$485 \quad 345$

56.6

Eight Vol

0.

1001

335

26.6

$1001 \quad 335$

105.7

Std Prb4

1.0

462

325

30.0

$462 \quad 325$

206.2

UCRW

3.05

71

69

9.05

$71 \quad 69$

67.3

TTWOB

$$
2.01
$$

224

200

17.7

$222 \quad 200$

118.8

Two-Dim Flow

1.002

$664 \quad 100$

10.7

$664 \quad 100$

47.3

Sec Side

0.5

743

500

43.4

$745 \quad 500$

343.1

TTQX1

1.005

223

200

23.8

223200

234.0

ATWS

150.0

$609 \quad 306$

53.2

$617 \quad 306$

404.9

* Actual time-step number

** Standard time-step number 
Essentially all category (1) and (2) items were expected to differ and no comparisons made for these quantities. Typical category (2) items are the time-zero acceleration pressure, enthalpy, mass, slip velocity, and control system convergence values.

The category 3 items may or may not differ. Those that do differ should not differ significantly. The acceptability criteria specified[6] was that overlay plots of results from the CDC and CRAY runs should not differ more than several pen widths. The correctness of the code installation is demonstrated by the graphs shown in Appendix A. The CRAY and CDC results have been overlaid in these graphs with the CDC results as the solid curve and the CRAY results as the dashed curve. In most cases, the curves are indistinguishable. In the worst case, i.e., the TTWOB case, the two calculations were similar in behavior but did not coincide precisely. The sample problems from the CRAY version which did not produce an exact match with the CDC version were using the iterative implicit numerical solution scheme. The time-step controller for the iterative implicit method did not always select the same criteria in the two versions because of machine related differences resulting in a slightly different set of time steps for the two versions. These differences can lead to results which are similar but not identical as shown below. Specifically, comparisons of the sample problem calculations resulted in the following:

\subsubsection{Standard Problem One}

Standard Problem One had only two major edits and no minor edits on the transmittal print file. The values in the major edits were the same (differing, at most, in the least significant digit) for both code versions. Both code versions executed the same number of time steps. Plots of minor edit variables from the CRAY version with corresponding major edit values from the CDC version as point data are shown in the Figures 1 through 6 in Appendix A. Standard Problem One executed successfully and met the acceptance criteria. 


\subsubsection{Standard Problem Five}

Values in the major edits were the same for both code versions. Both code versions executed the same number of time steps. Overlay plots from minor edit variables from both the CRAY and CDC code versions are shown in Figures 7 through 12 in Appendix A. Standard Problem Five executed successfully and met the acceptance criteria.

\subsubsection{Eight Volume Sample Problem}

Values in the major edits were the same for both code versions. Both code versions executed the same number of time steps. Overlay plots from minor edit variables from both the CRAY and CDC code versions are shown in Figures 13 and 14 in Appendix $A$. The eight volume sample problem executed successfully and met the acceptance criteria.

\subsubsection{Standard Problem Four}

Values in the major edits were the same for both code versions. Both code versions executed the same number of time steps. Plots of minor edit variables from the CRAY version with corresponding major edit values from the CDC version as point data are shown in the Figures 15 through 19 in Appendix A. Standard Problem Four executed successfully and met the acceptance criteria.

\subsubsection{Uncontrolled Rod Withdrawal}

Values in the major edits were the same for both code versions. Both code versions executed the same number of time steps. Overlay plots from minor edit variables from both the CRAY and CDC code versions are shown in Figures 20 through 26 in Appendix A. The uncontrolled rod withdrawal problem executed successfully and met the acceptance criteria. 


\subsubsection{Turbine Trip Without Bypass (Point Kinetics)}

The transient calculations, as shown in the TTWOB figures, were similar for the CDC and CRAY code versions but did not overlay each other exactly. The CRAY version took two more time steps than did the CDC version, one occurring early in the calculation and the second late in the calculation. Overlay plots from minor edit variables from both the CRAY and CDC code versions are shown in Figures 27 and 28 in Appendix A. The turbine trip without bypass problem with point kinetics executed successfully but did not meet the acceptance criteria.

It was suspected that the CDC case was marginally converged and that smaller time steps would produce converged solutions on both the CRAY and CDC machines. Consequently, the problem was rerun on both the CDC and CRAY versions of RETRAN-02/MOD004. Both code versions executed the same number of time steps for this case. Overlay plots of the results from this case are shown in Figures 29 through 32 in Appendix A. The modified turbine trip without bypass problem with piont kinetics executed successfully and met the acceptance criteria.

\subsubsection{Two-Dimensional Flow Field}

Values in the major edits were the same for both code versions. Both code versions executed the same number of time steps. No plots were made of the results from this test problem since it converges to a steady-state condition. The two-dimensional flow field problem executed successfully and met the acceptance criteria.

\subsubsection{Secondary-Side Sample Problem}

Values in the major edits were the same for both code versions. The CRAY version took two fewer time steps than did the CDC version. The difference in time steps occurred in the first 50 time steps where the time steps were being limited by different criteria in the two versions. Overlay plots from minor 
edit variabies from both the CRAY and CDC code versions are shown in Figures 35 through 44 in Appendix $A$. The secondary-side sample problem executed successfully and met the acceptance criteria.

\subsubsection{Turbine Trip Without Bypass (One-Dimensional Kinetics)}

Values in the major edits were the same for both code versions. Both code versions executed the same number of time steps. Overlay plots from minor edit variables from both the CRAY and CDC code versions are shown in Figures 45 through 52 in Appendix A. The turbine trip without bypass problem with one-dimensional kinetics executed successfully and met the acceptance criteria.

\subsubsection{Anticipated Transient Without Scram}

Calculated results were nearly the same for both code versions. The CRAY version took eight fewer time steps than did the CDC version. The difference in time steps occurred after 81.0 seconds into the transient where the time steps were being limited by different criteria in the two versions. The ATWS case has a set of relief valves which cycle during the transient. One more reset occurs in the CRAY Calculation after 81.0 seconds into the calculation leading to the differences observed in the junction 90 behavior. Overlay plots from minor edit variables from both the CRAY and CDC code versions are shown in Figures 53 through 66 in Appendix A. The anticipated transient without scram problem executed successfully and met the acceptance criteria.

\subsection{RESTART}

The restart option was validated by running the restart sample problems given in Reference 1 for test cases 5 and 6 above and the acceptability of the test case results validated by comparing the restart edits with the original cases. The UCRW restart case utilized the generalized restart option in the code. Results from this case were the same as the values listed in Volume 3 of the code documentation. [1] The TTWOB (Point Kinetics) case was also restarted. 
The restart calculation for the TTWOB case was similar to the base case but did not duplicate it exactly. Both the UCRW restart case and the TTWOB restart case executed successfuity. The UCRW restart case met the acceptance criteria but the TTWOB restart did not meet the acceptance criteria for the same reasons as the base case (the solution was not converged with respect to the time-step size). A restart case was run using the modified TTWOB case which did meet the acceptance criteria.

\subsection{REEDIT}

The reedit test cases given in Reference 1 were run. Successful execution and validation of the test cases was demonstrated by retrieving and printing the previously archived results from the appropriate restart data files. The reedit cases were successful and met the acceptance criteria.

\subsection{PLOT}

Two of the plot sample problems given in Reference 1 were run. Dummy routines to satisfy the Calcomp external references were provided and the plot feature validated by examining tabular output for the curves normally plotted. The RETRAN-02 plot option successfully executed the test cases and results met the acceptance criteria. 


\subsection{REFERENCES}

1. McFadden, J. H., et a1, "RETRAN-02 - A Program for Transient Therma1Hydraulic Analysis of Complex Fluid Flow, NP-1850-CCM-A, R4, Volumes 1-4, Electric Power Research Institute, June 1987.

2. Project Requirements Document for the RETRAN-02 Modifications, LANL-PRD-001, Revision 0, February, 1990.

3. UPDATE Reference Manua7, Cray Research, Inc., SR-0013, Revision J, November 1989.

4. CTSS Mini-Reference, Los Alamos National Laboratory, CIC \#205, August, 1988.

5. FORTRAN (CFT) Reference Manual, Cray Research, Inc., SR-0009, Revision L, July 1986.

6. Software Design Document for the RETRAN-02 Cray Installation, LANL-SDD-001, Revision 0, March, 1990. 


\section{APPENDIX A}

\section{SAMPLE PROBLEM DATA OVERLAY PLOTS}

The results from nine of the sample problem cases were checked against the respective sample problem output files from the transmittal tape by making overlay plots for several of the minor edit variables from the CDC and CRAY versions. The CRAY and CDC results have been overlaid in the figures in this Appendix with the CDC results as the solid curve and the CRAY results as the dashed curve. The sample problem output from the transmittal tape did not contain minor edits for two of these cases, i.e., Standard Problem One and Standard Problem Four. Point values from the major edits in these latter cases were included on the data plots without connecting curves ( $X$ markers). The figures from each of the sample problems are as follows:

Standard Problem One

Standard Problem Five

Eight Volume Problem

Standard Problem Four

Uncontrolled Rod Withdrawal

Turbine Trip Without Bypass (Point Kinetics)

Secondary-Side Sample Problem

Turbine Trip Without Bypass (1-D Kinetics)

Anticipated Transient Without Scram
Figures $1-6$

Figures $7-12$

Figures $13-14$

Figures $15-19$

Figures $20-26$

Figures $27-34$

Figures $35-44$

Figures $45-52$

Figures $53-66$ 
Figure 1

Standard Problem One

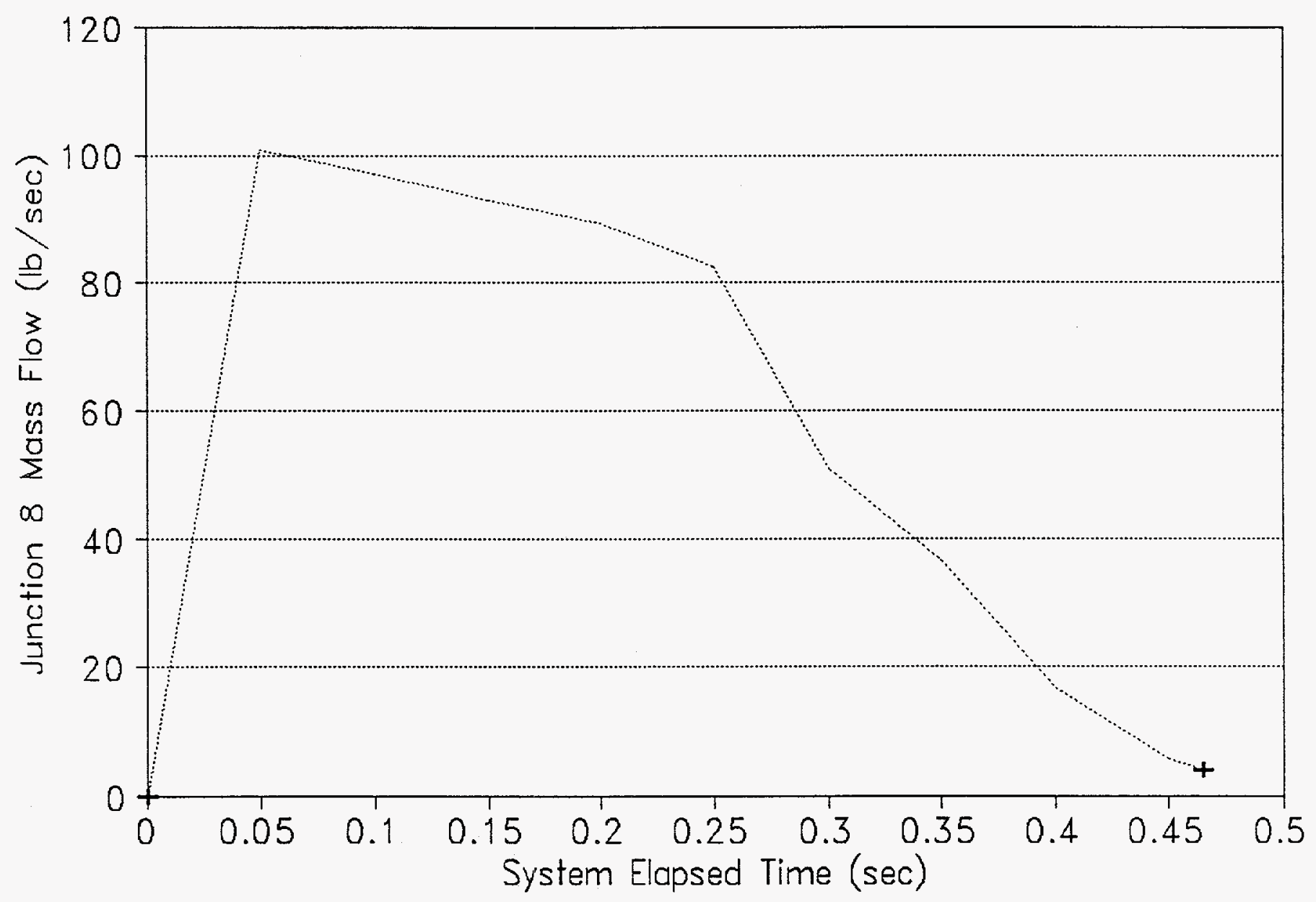


Figure 2

Standard Problem One

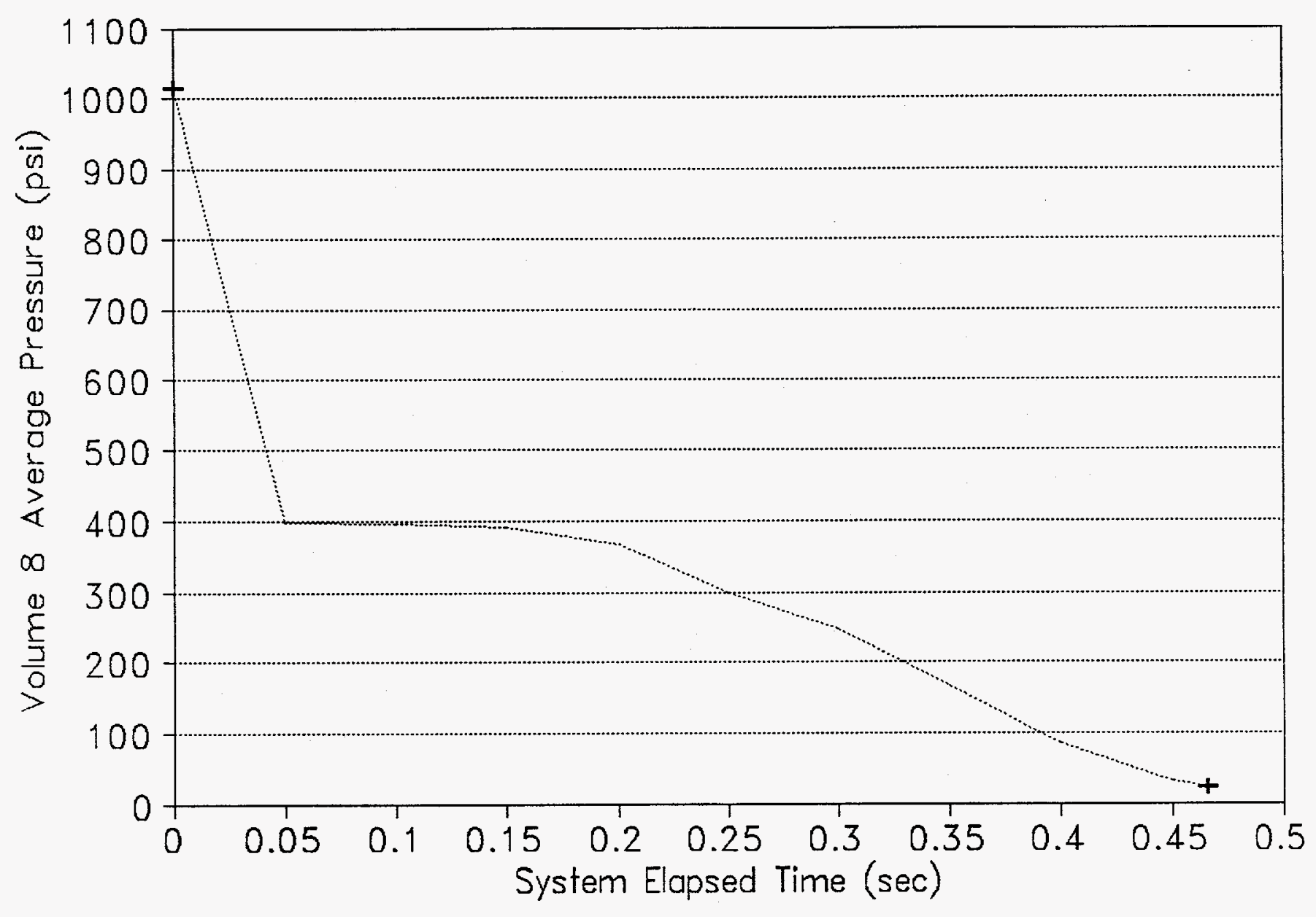


Figure 3

Standard Problem One

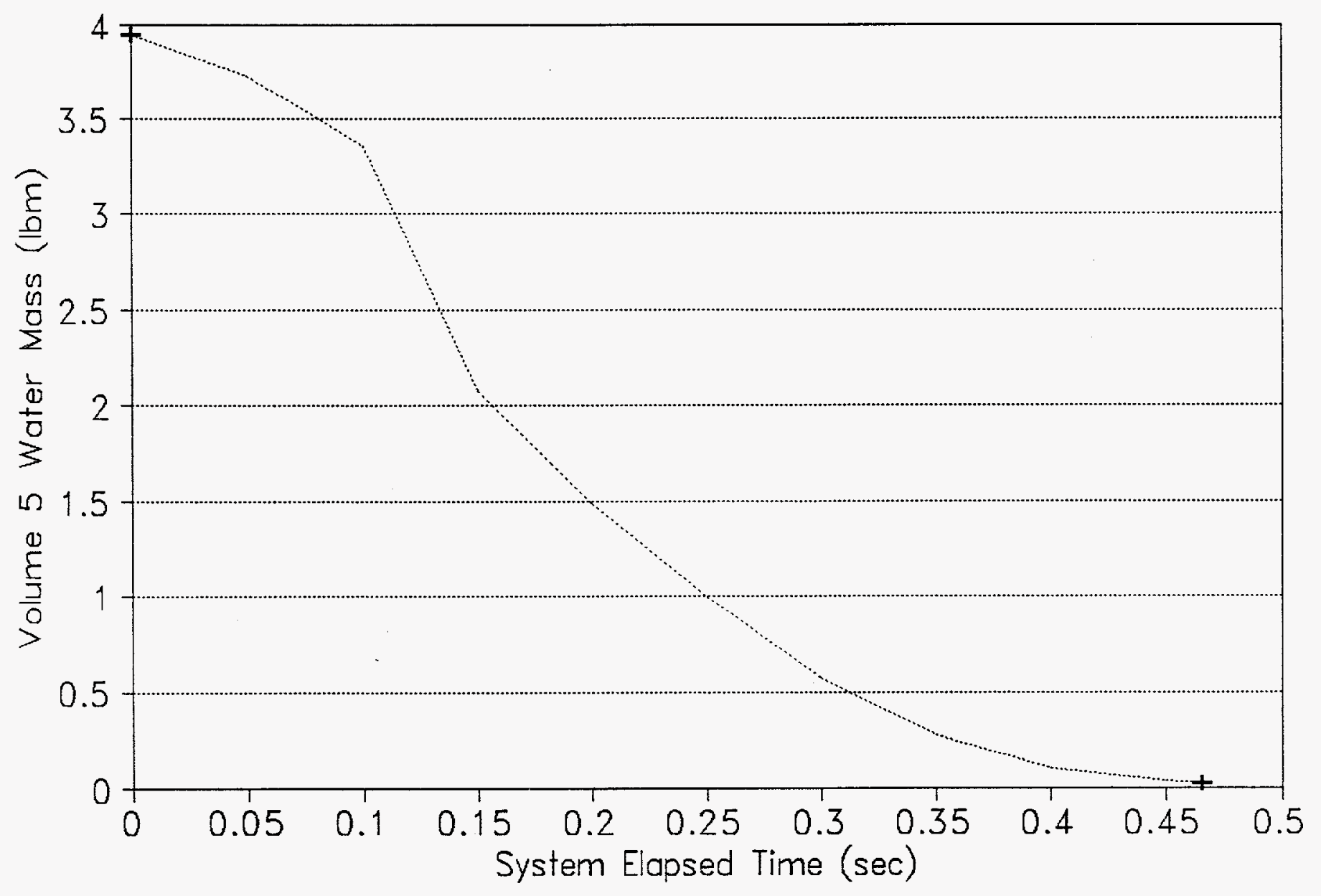

A- 4 
Figure 4

\section{Standard Problem One}

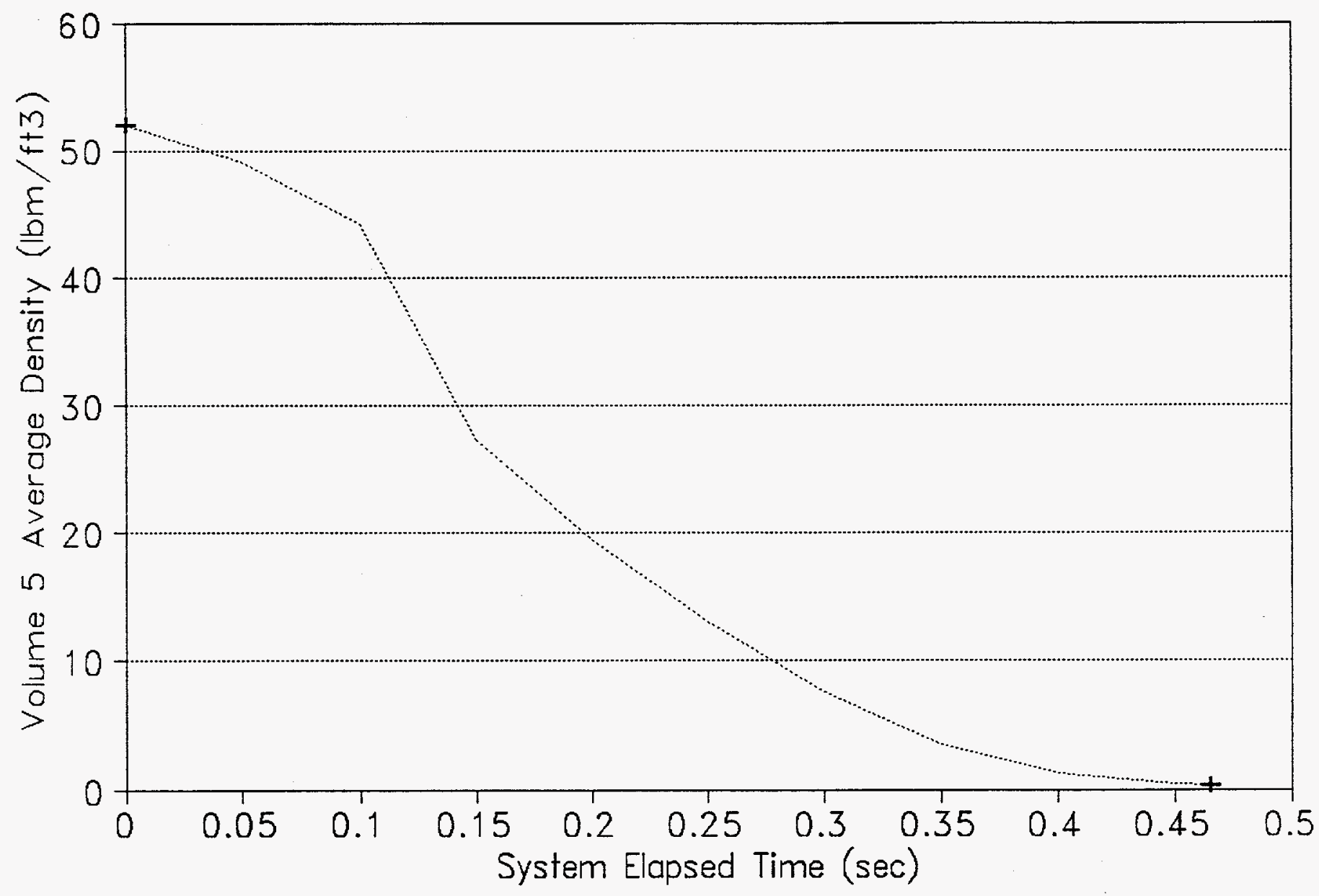


Figure 5

\section{Standard Problem One}

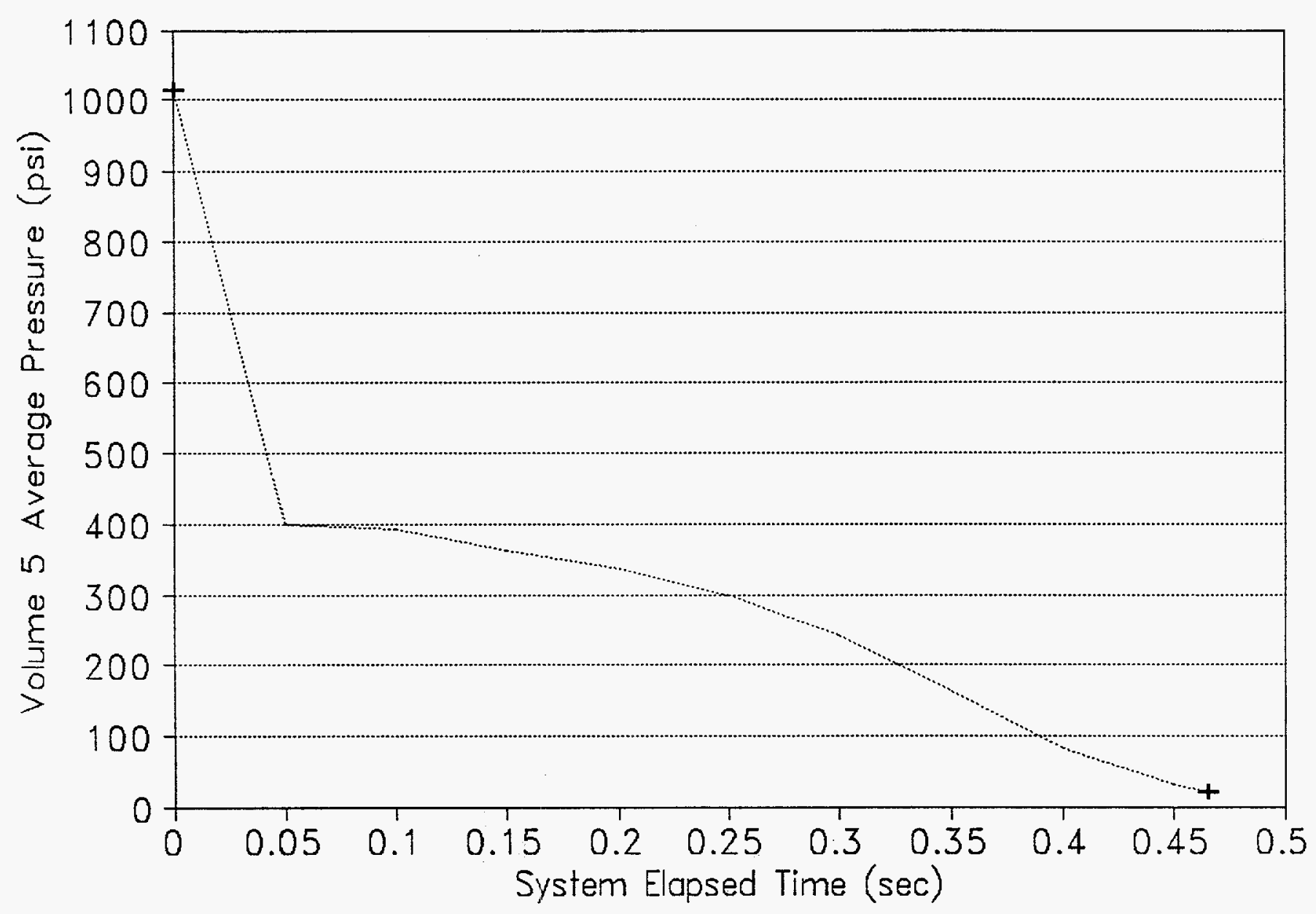


Figure 6

\section{Standard Problem One}

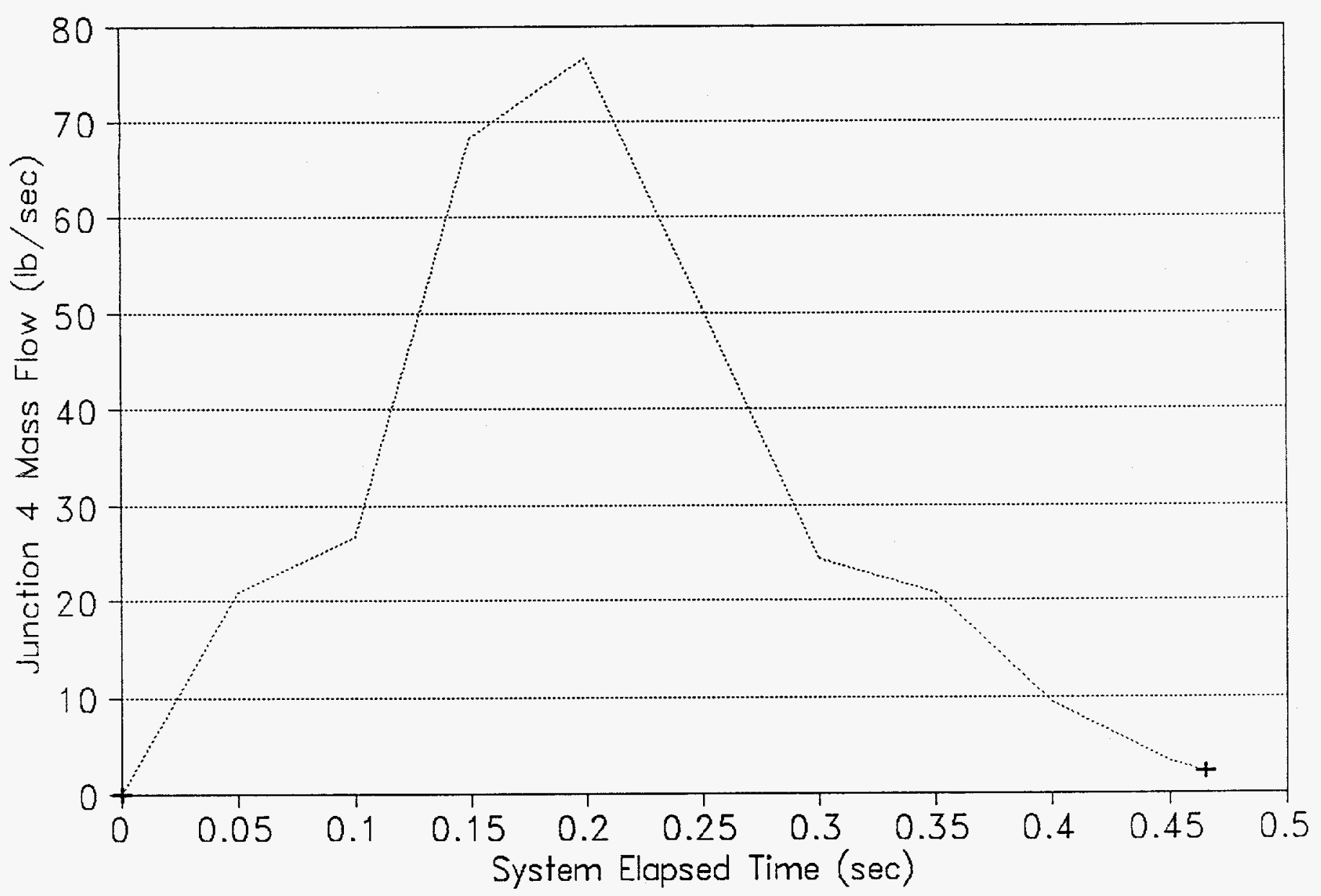


Figure 7

\section{Standard Problem Five}

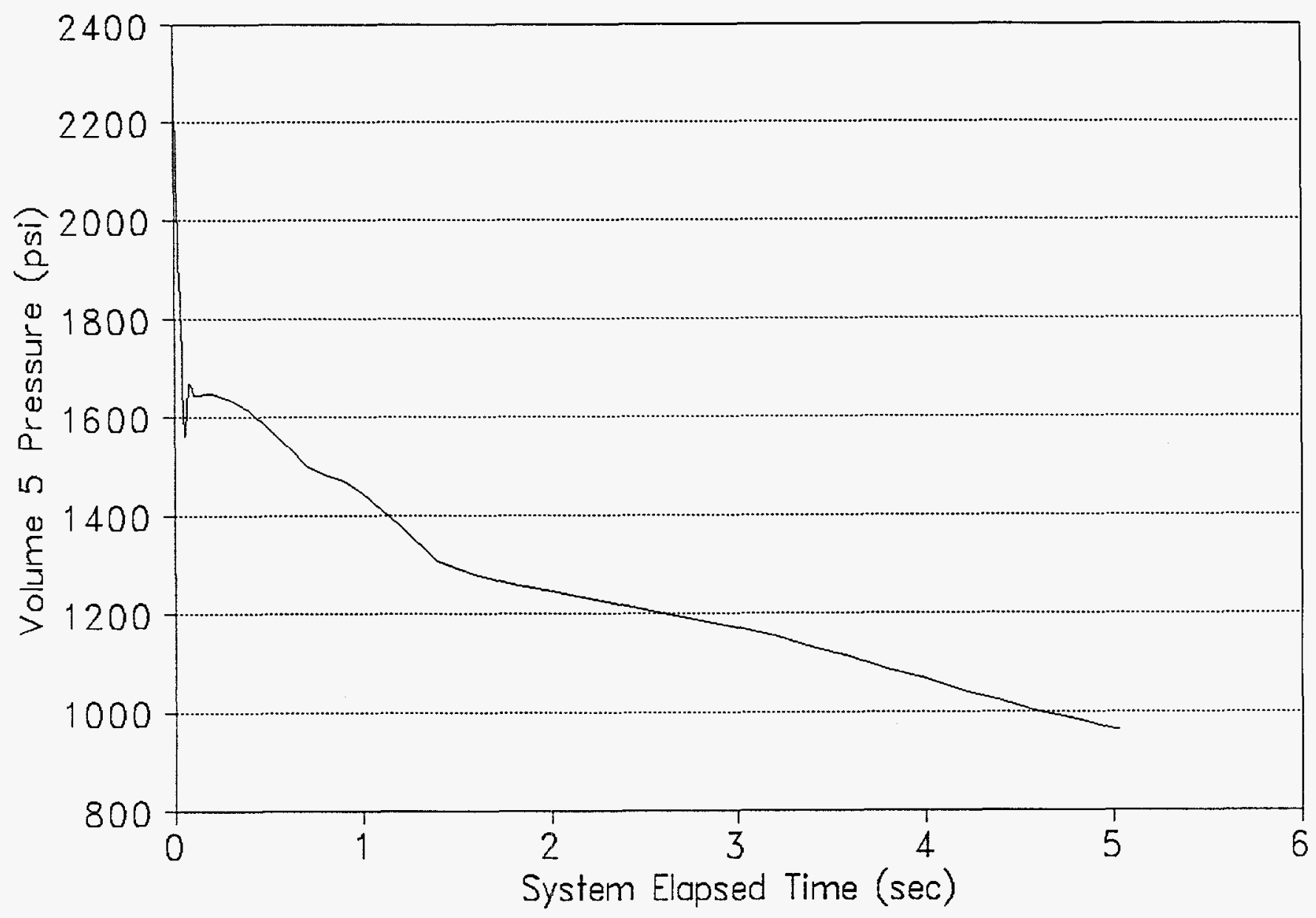


Figure 8

Standard Problem Five

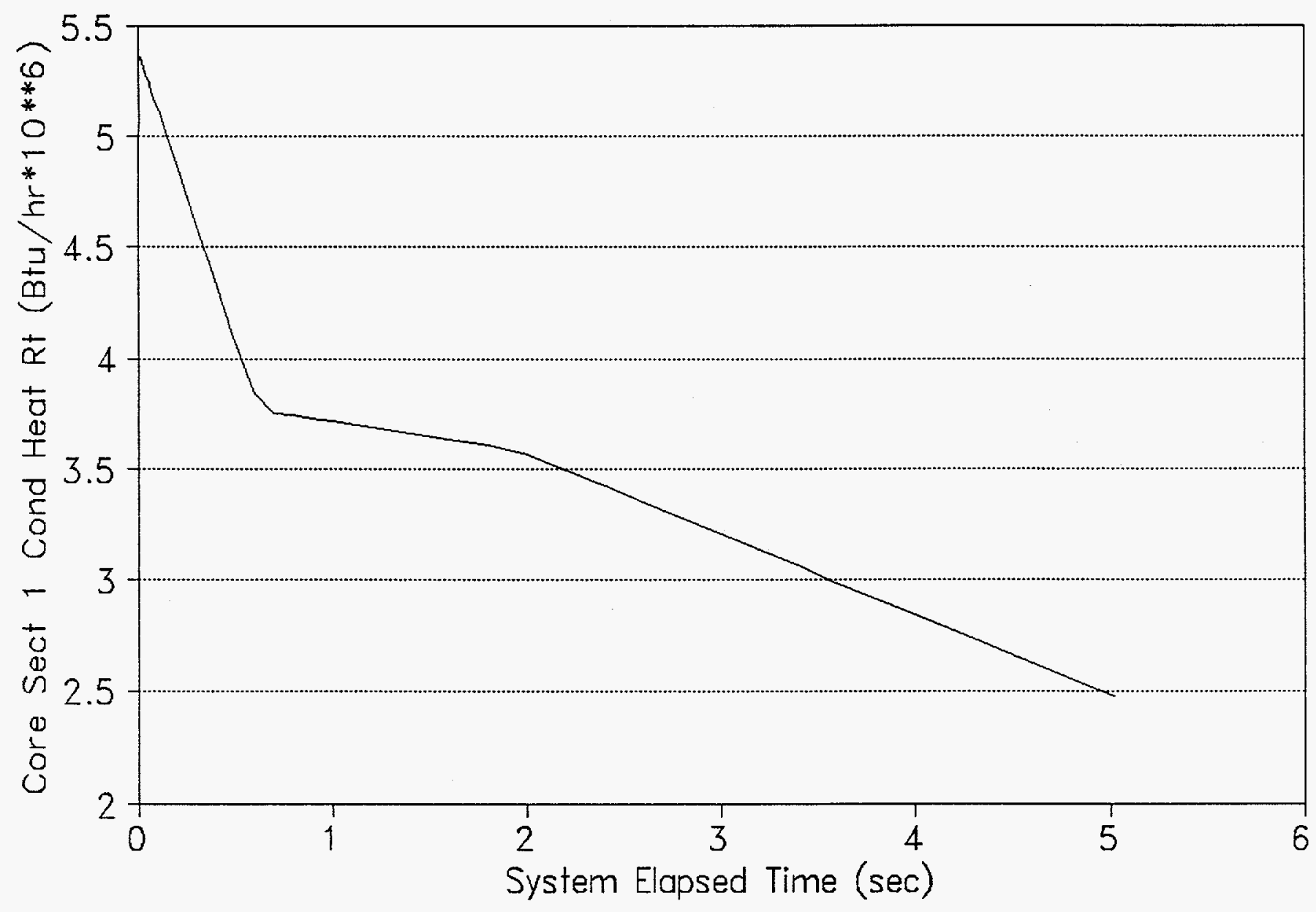


Figure 9

\section{Standard Problem Five}

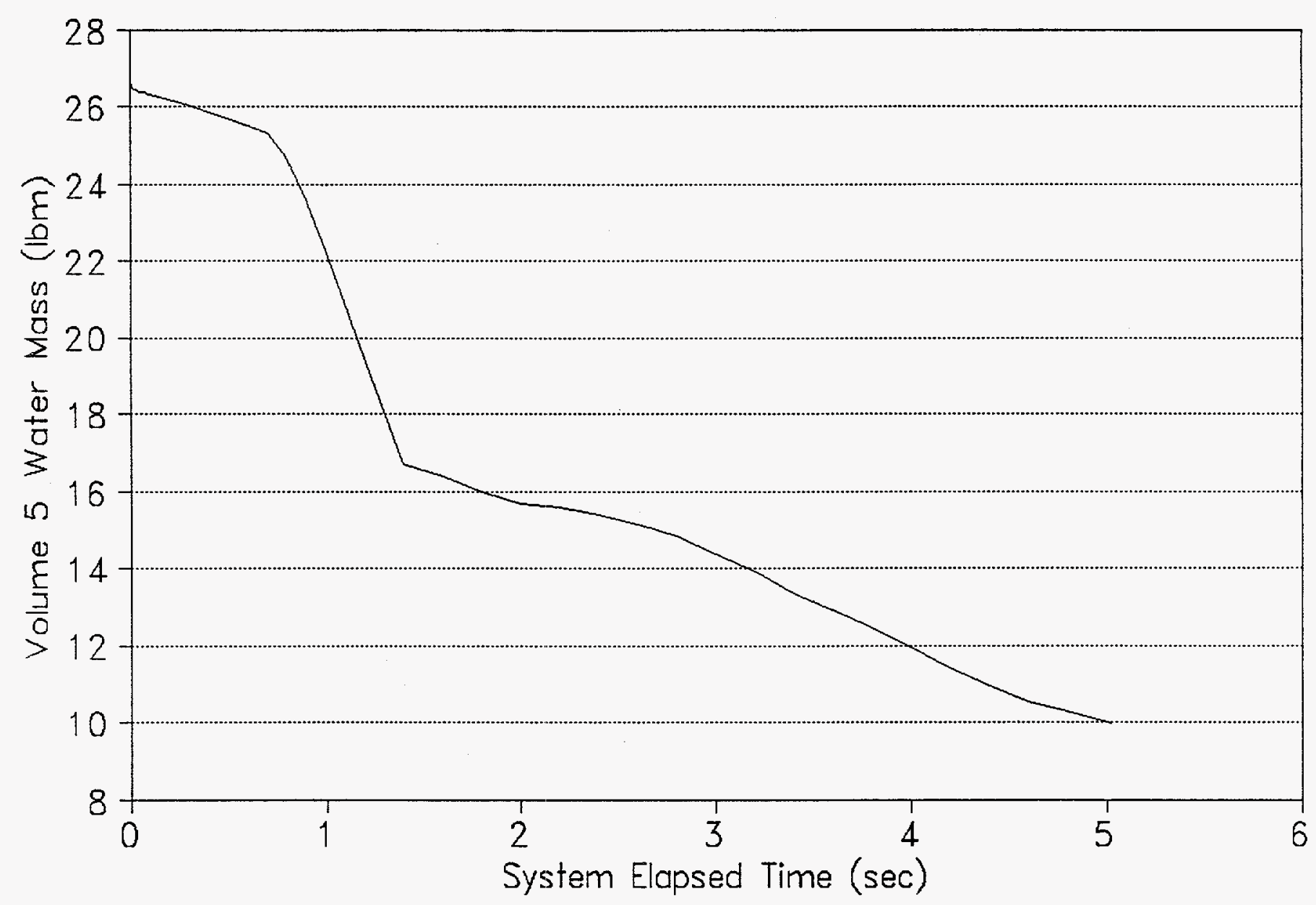


Figure 10

Standard Problem Five

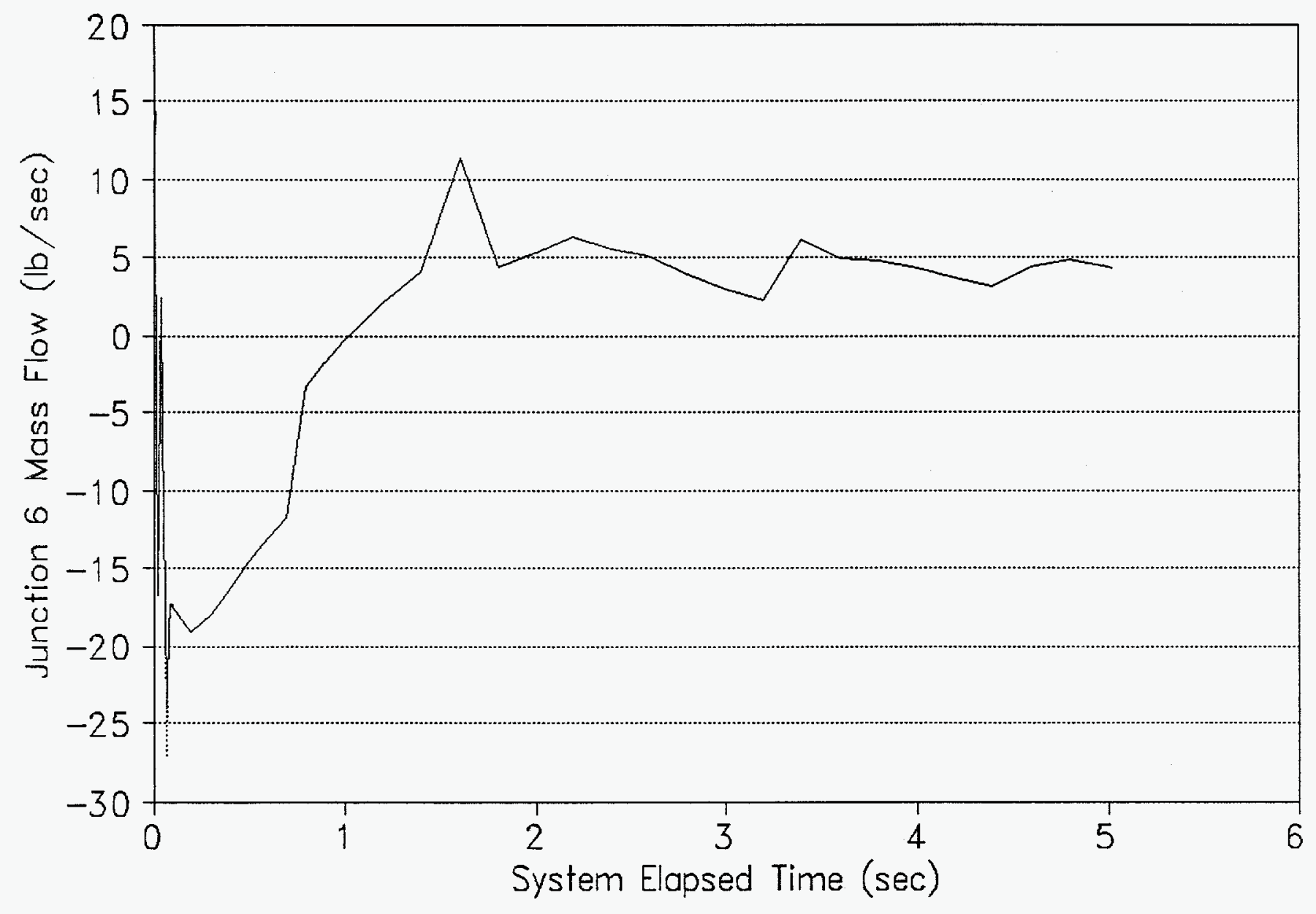


Figure 11

Standard Problem Five

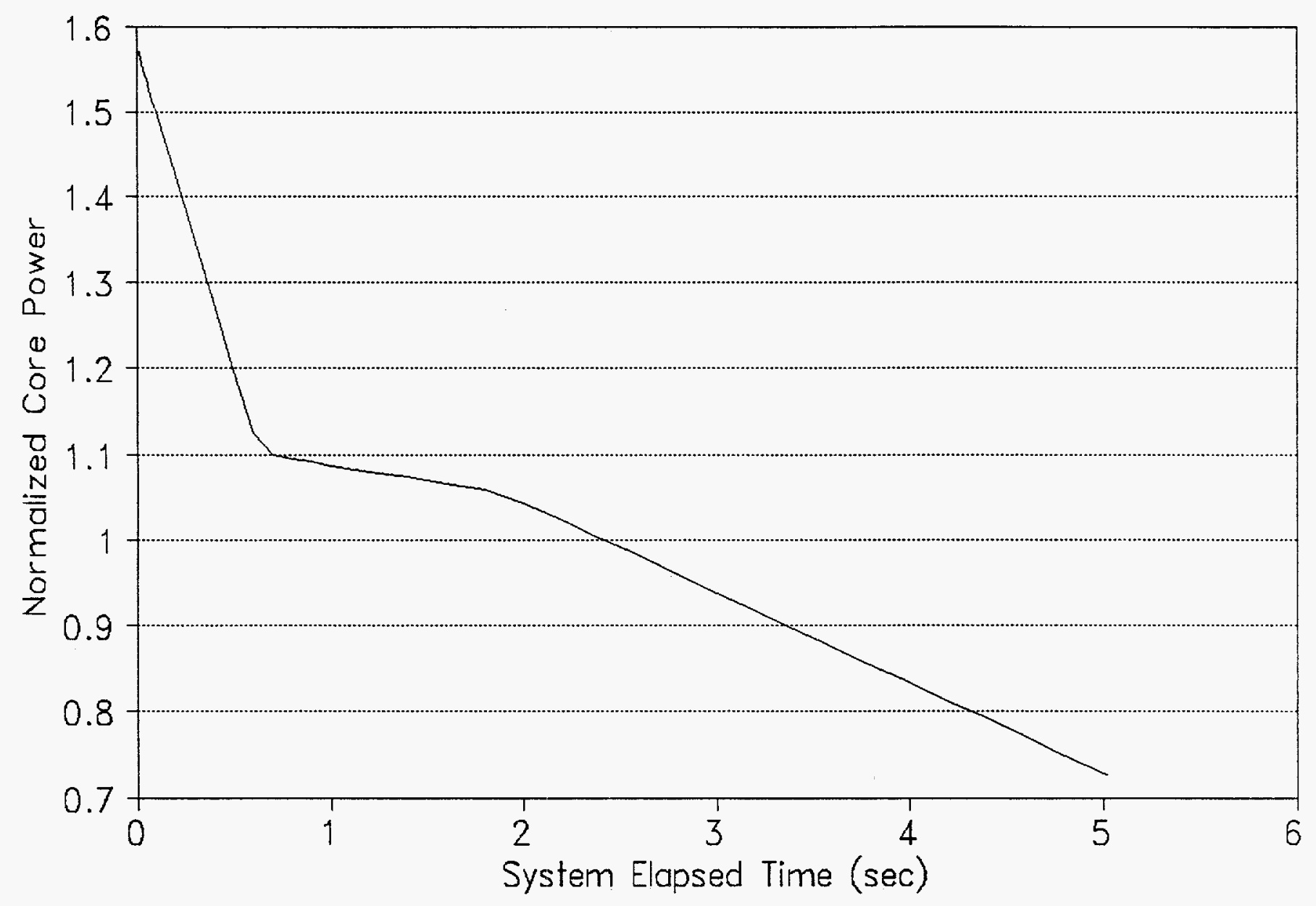


Figure 12

\section{Standard Problem Five}

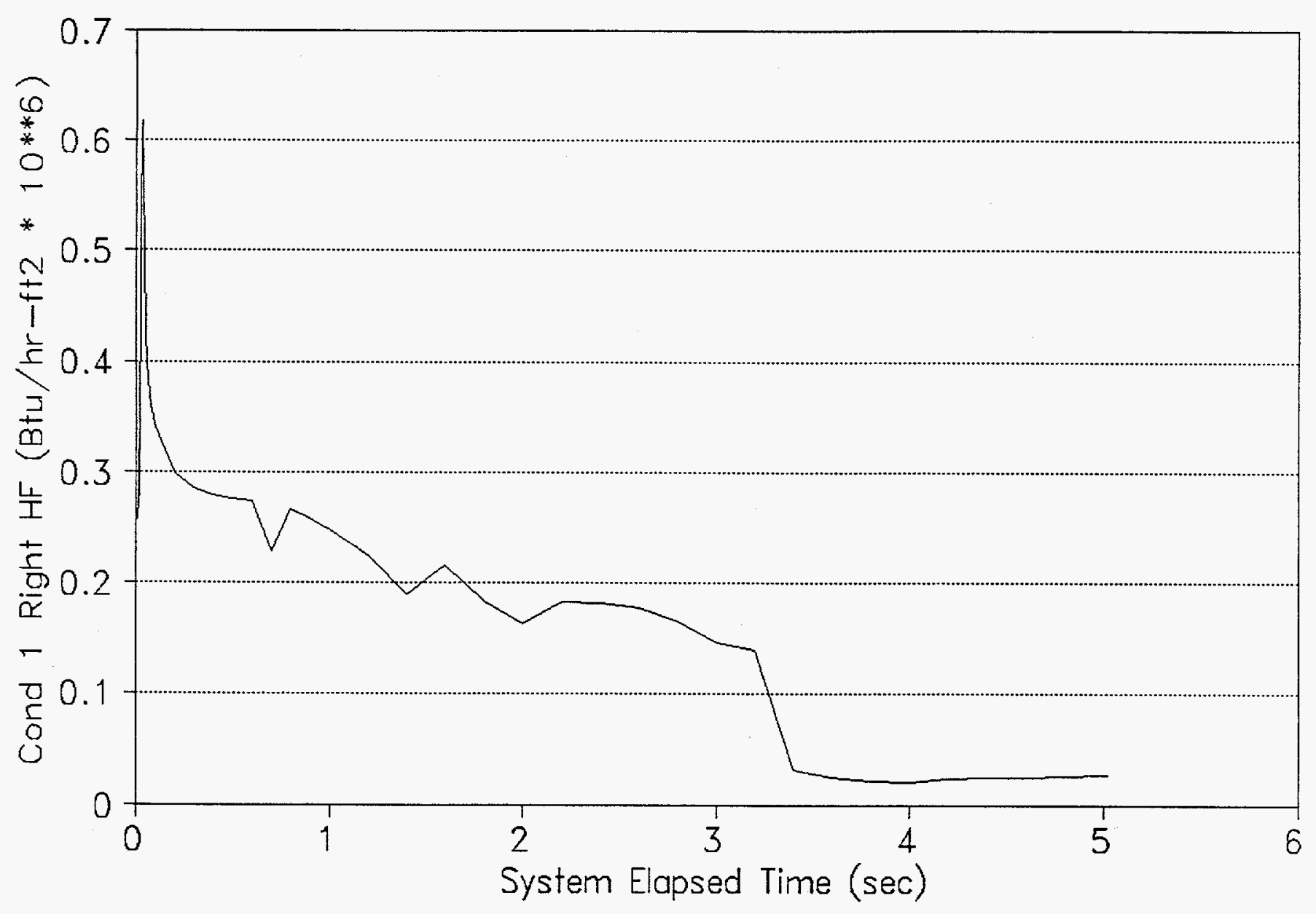


Figure 13

Volume 8

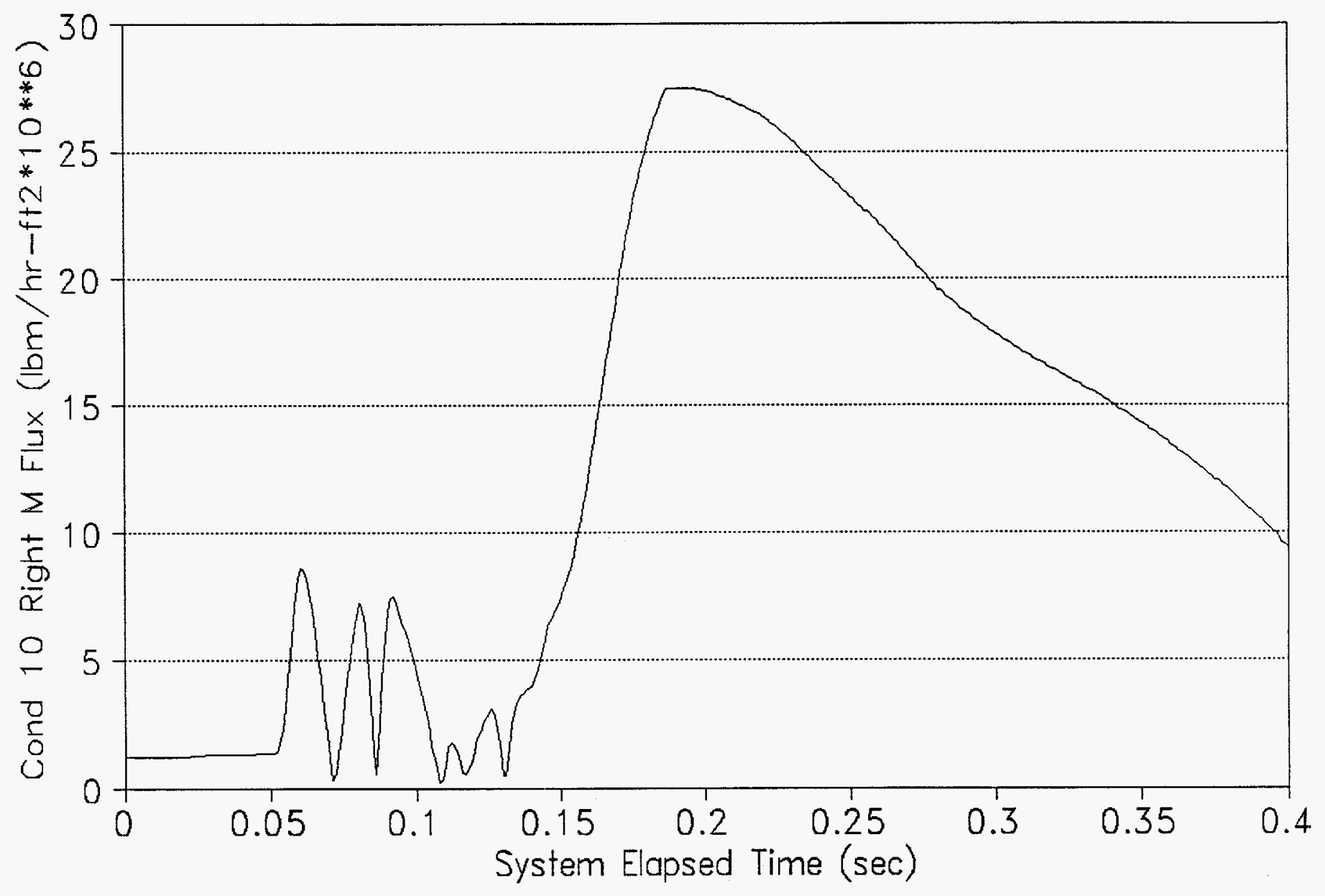

A-14 
Figure 14

Volume 8

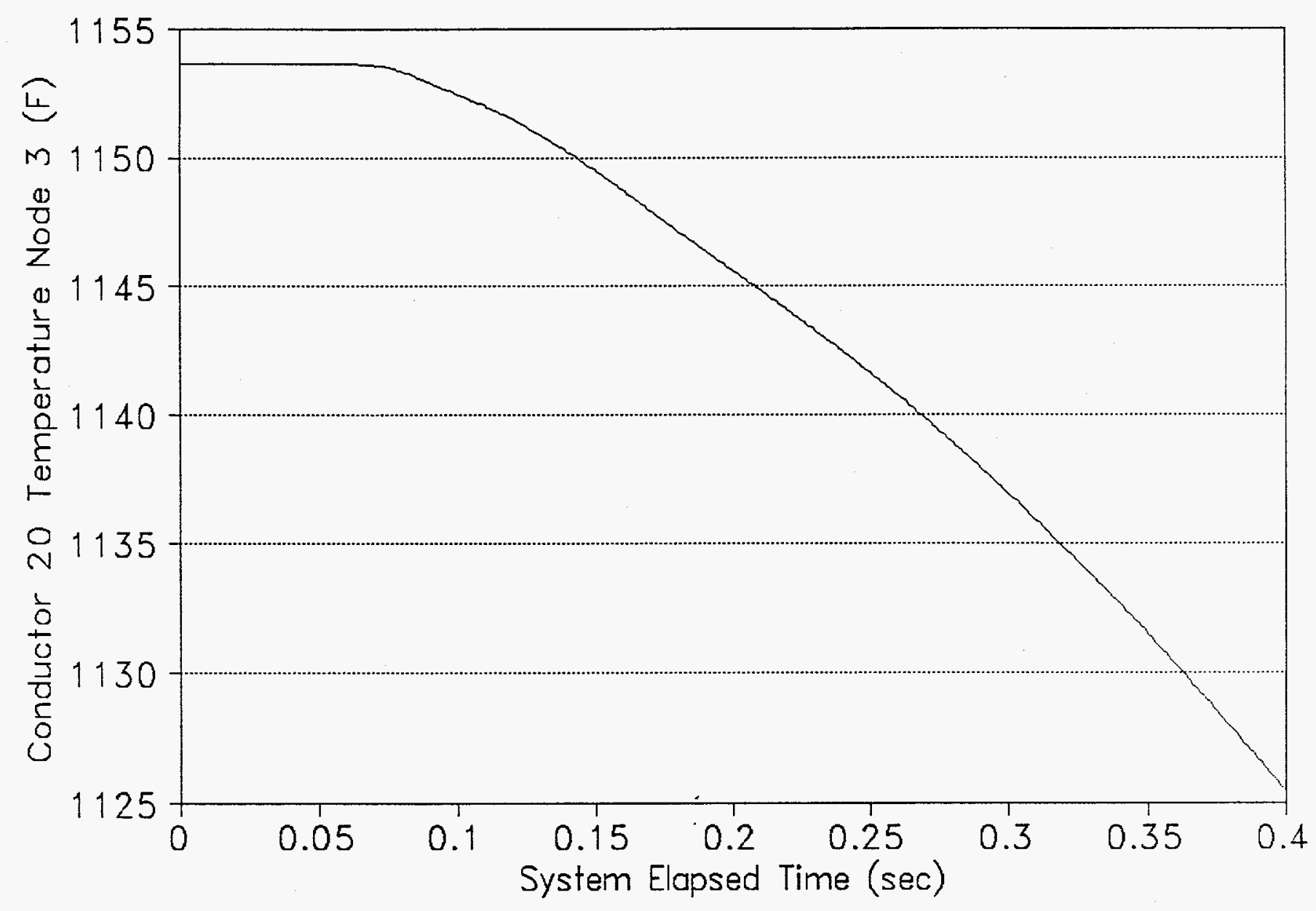


Figure 15

\section{Standard Problem Four}

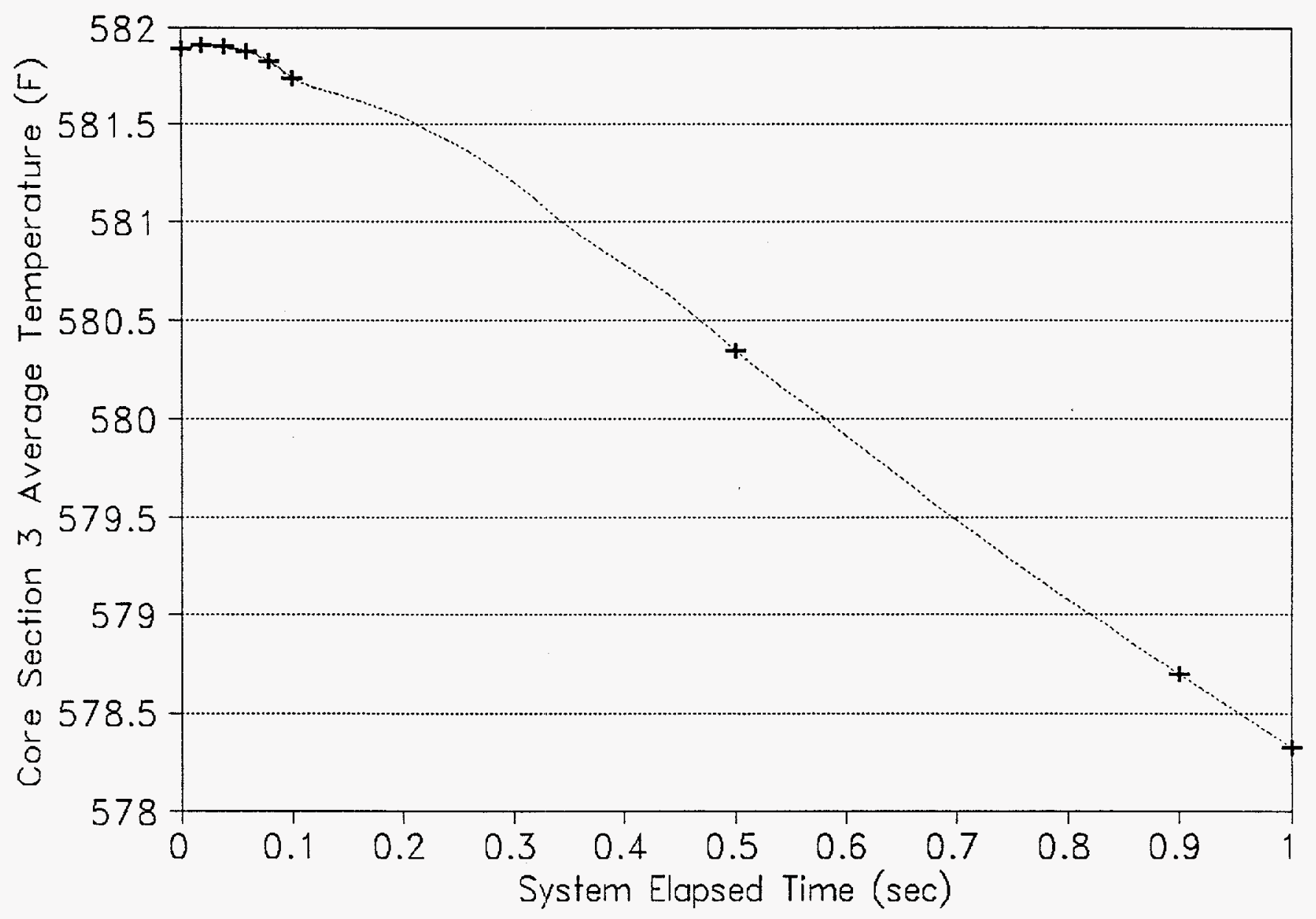


Figure 16

Standard Problem Four

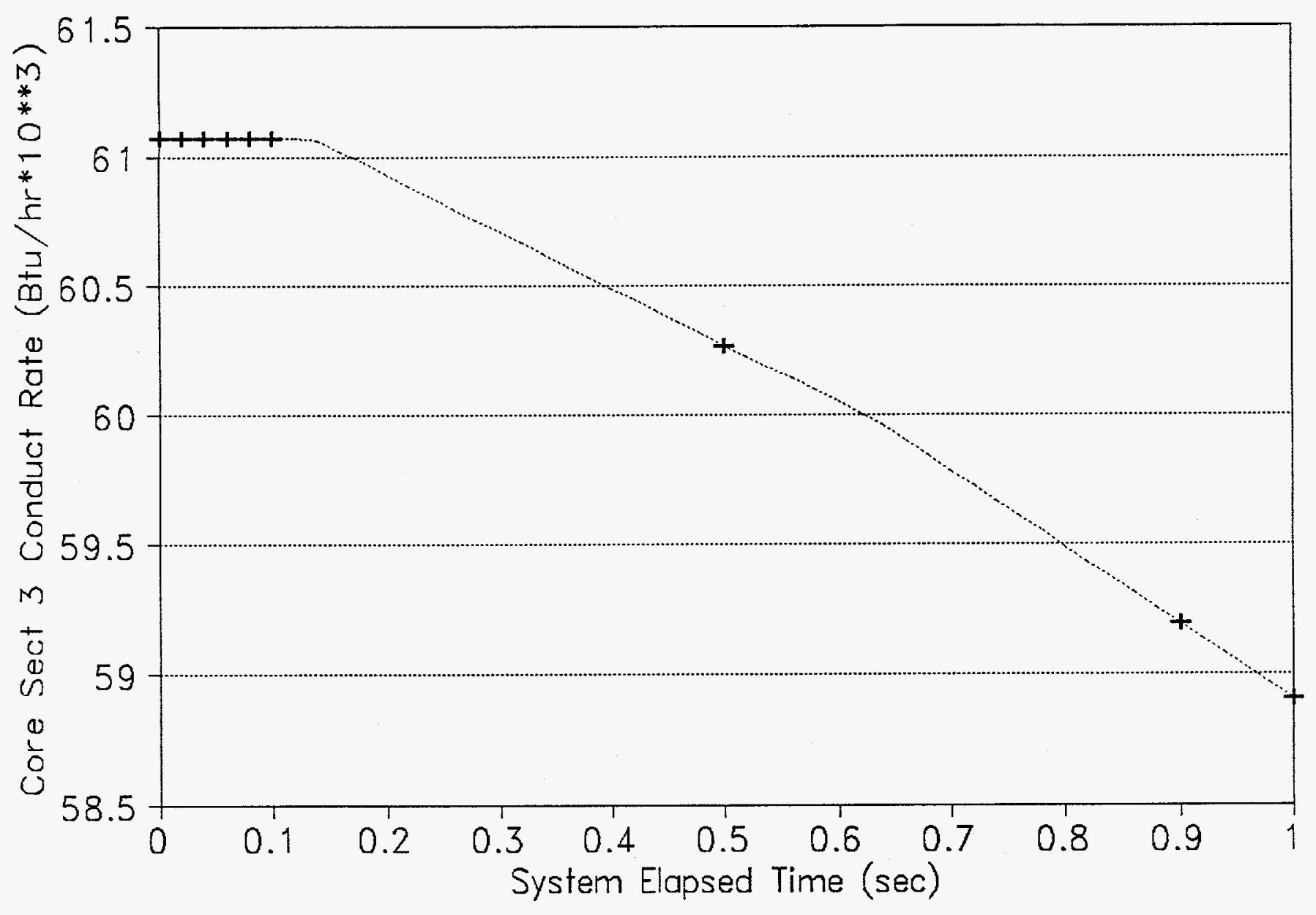


Figure 17

\section{Standard Problem Four}

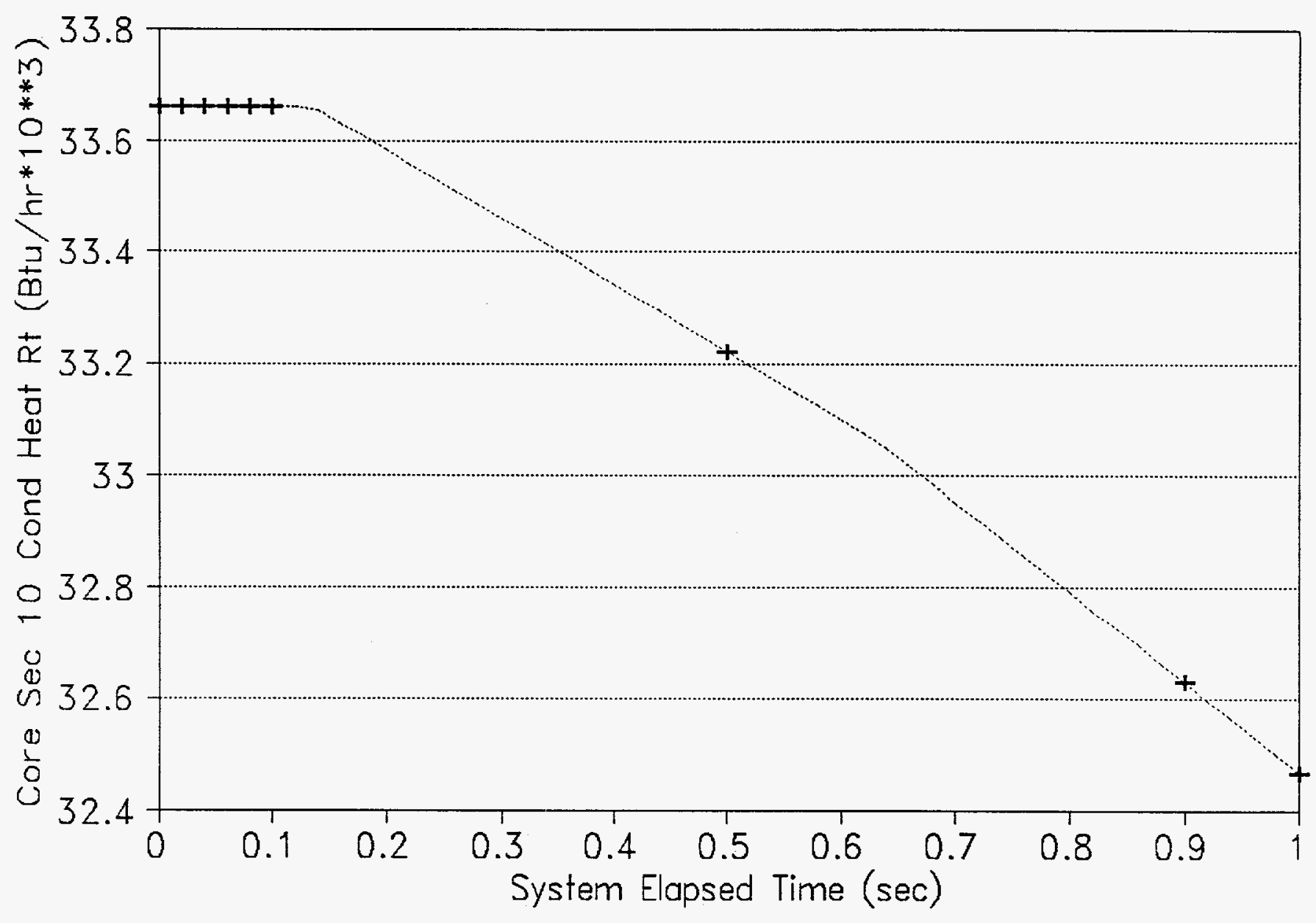


Figure 18

Standard Problem Four

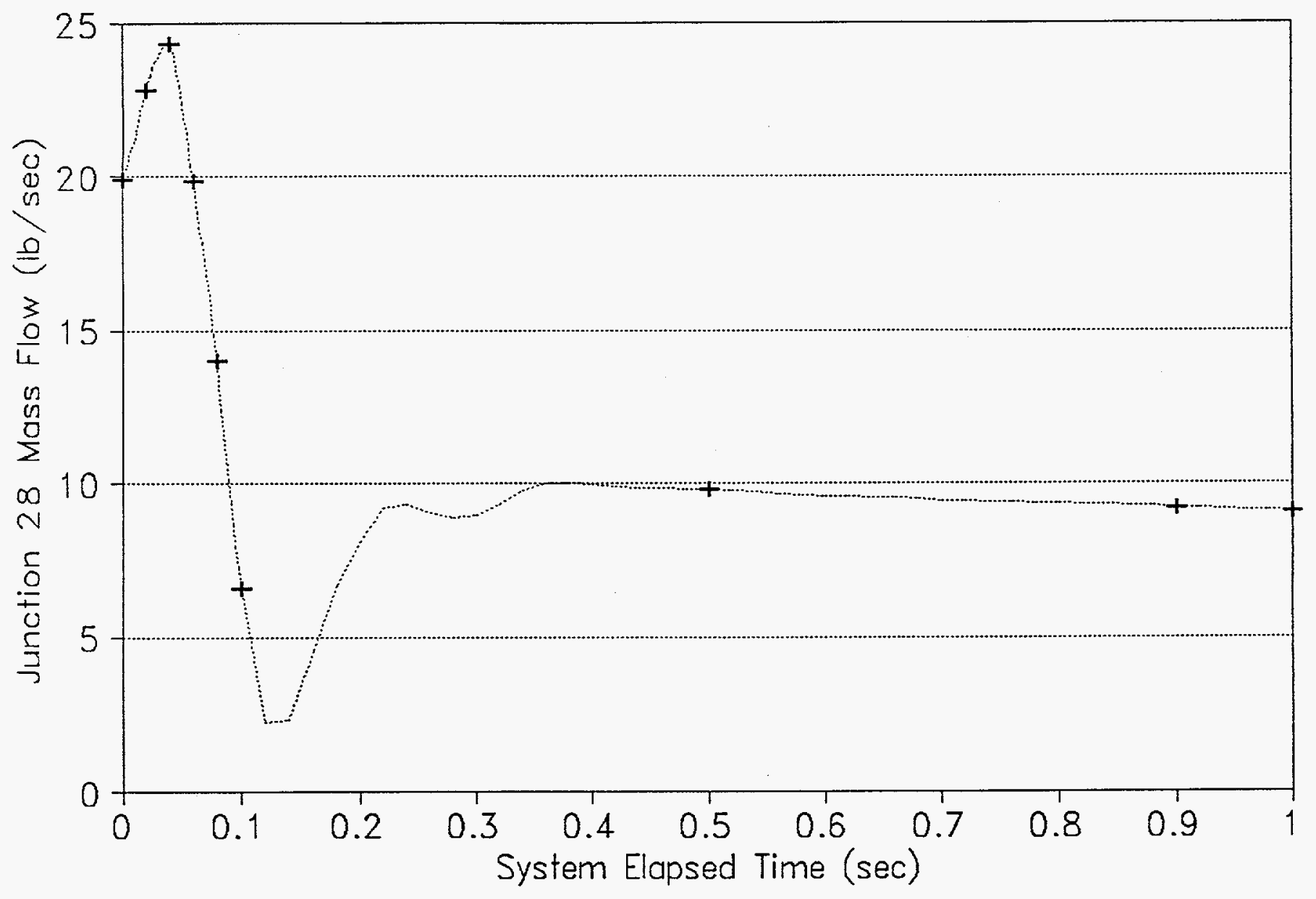


Figure 19

Standard Problem Four

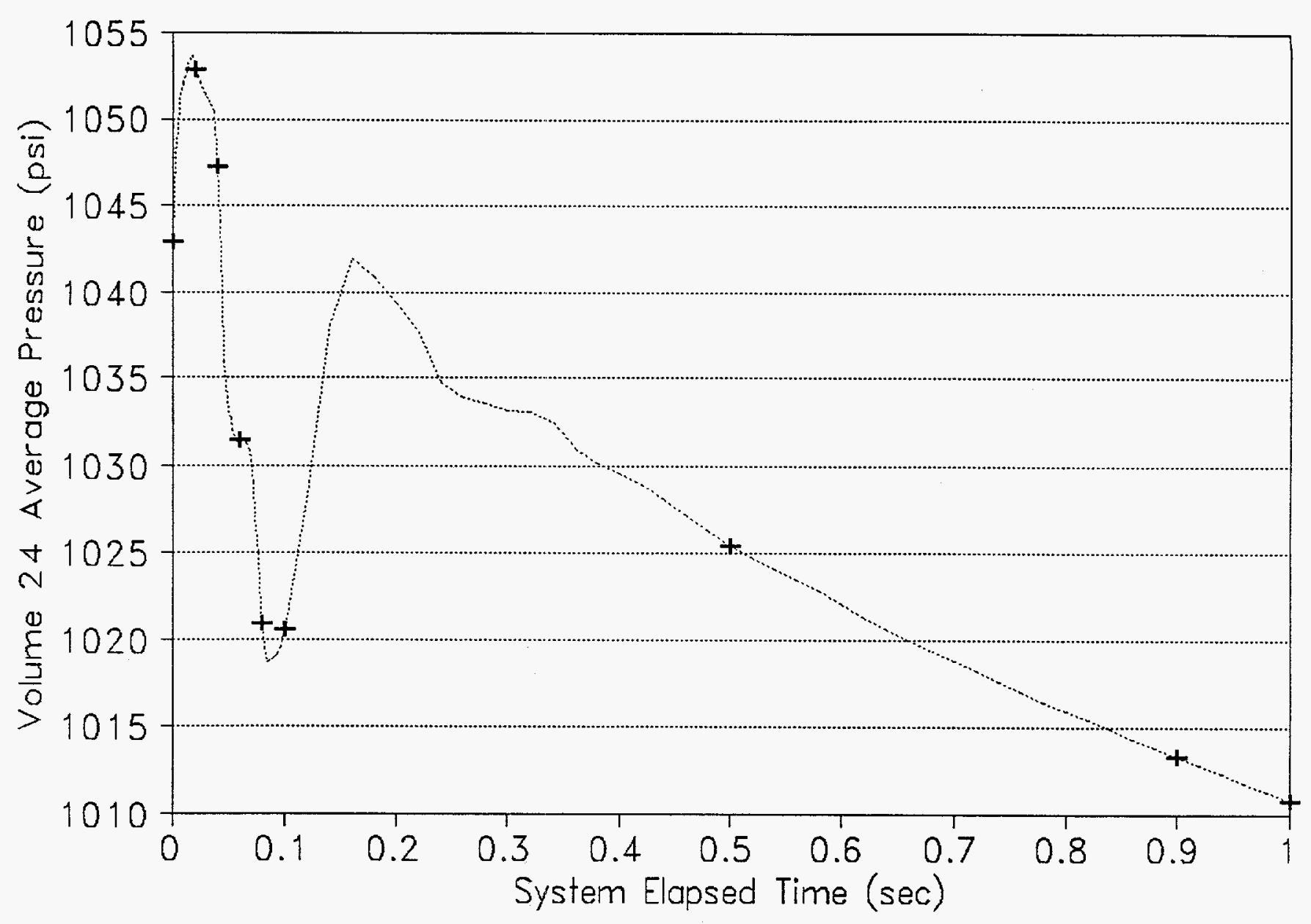


Figure 20

Uncontrolled Rod Withdrawal

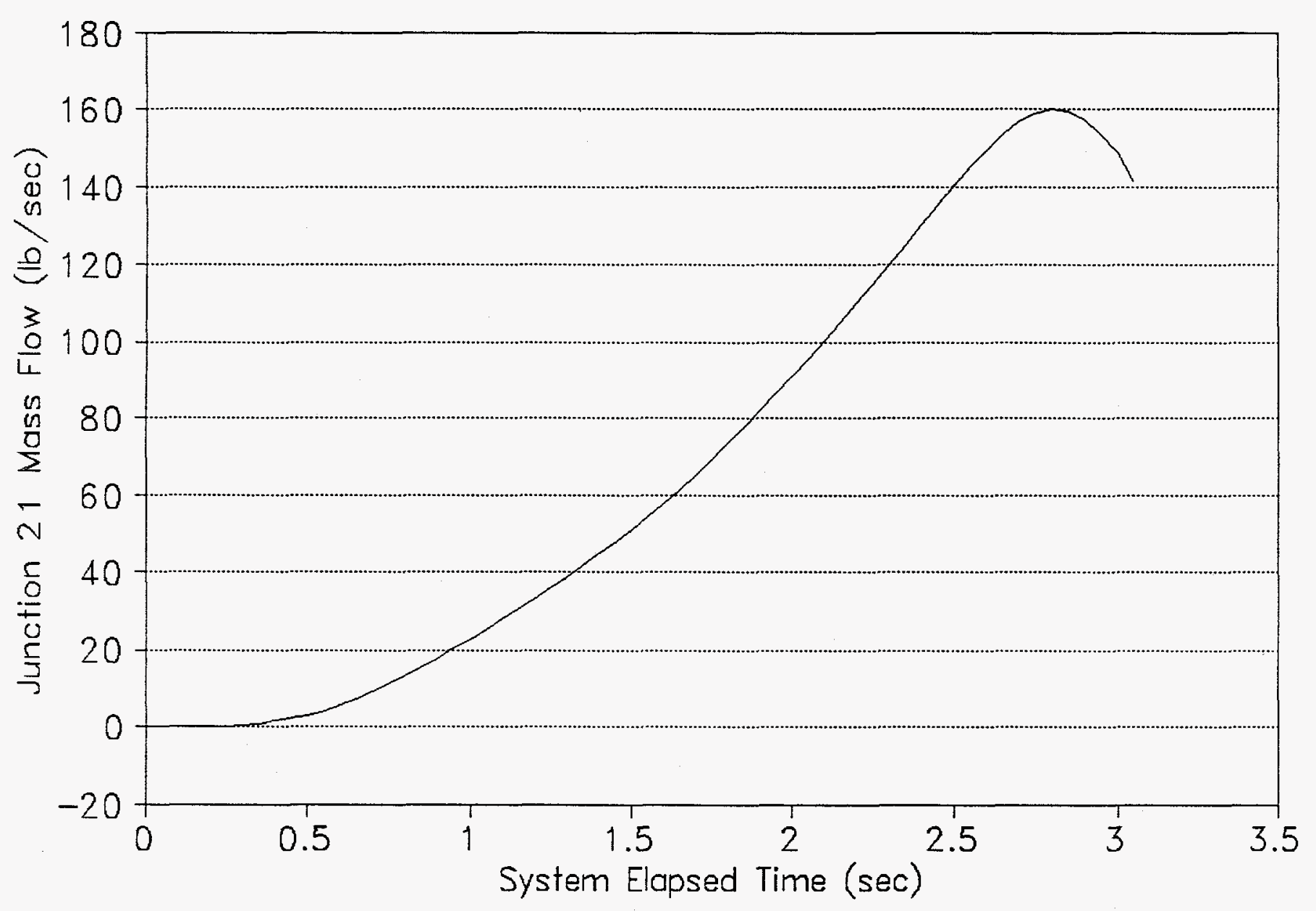


Figure 21

Uncontrolled Rod Withdrawal

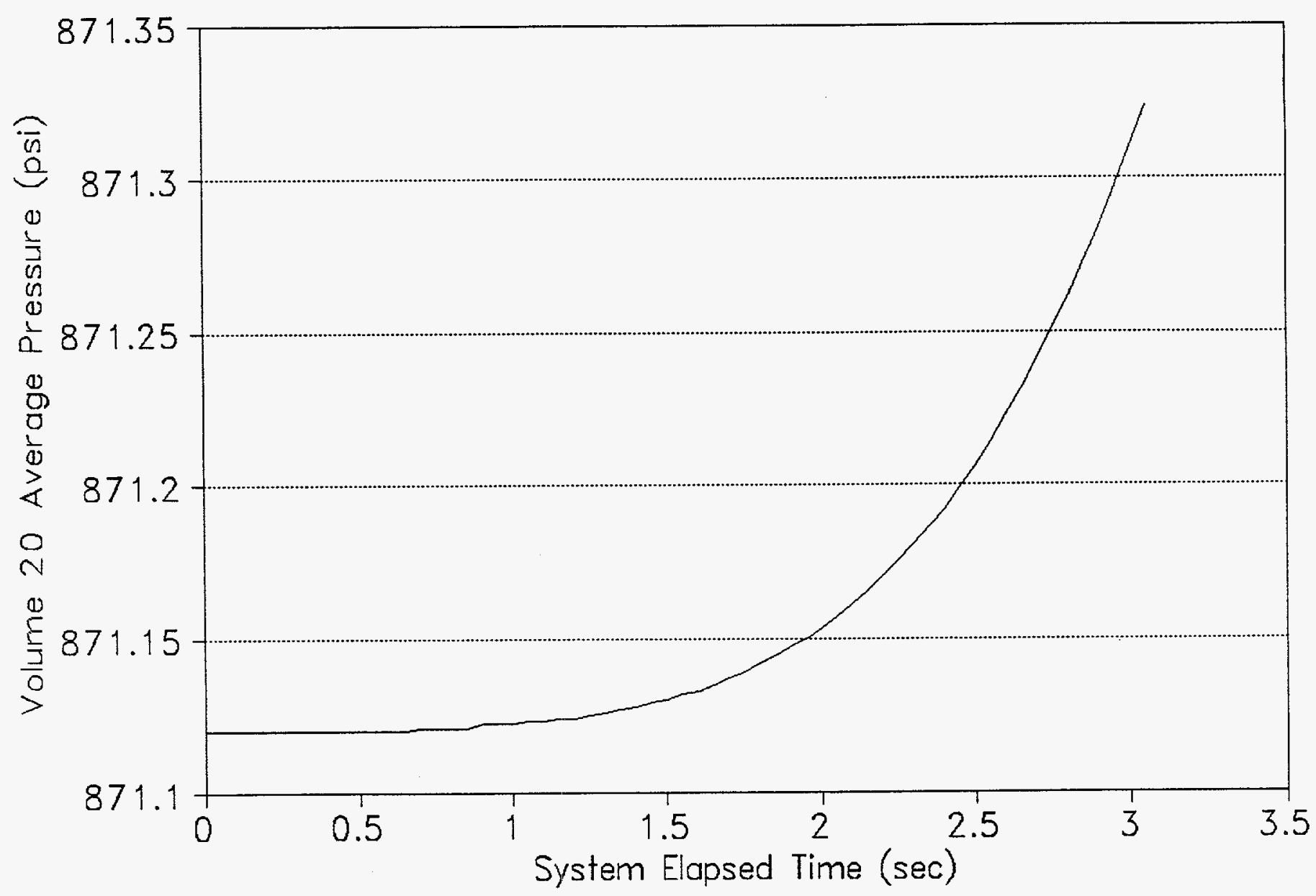


Figure 22

Uncontrolled Rod Withdrawal

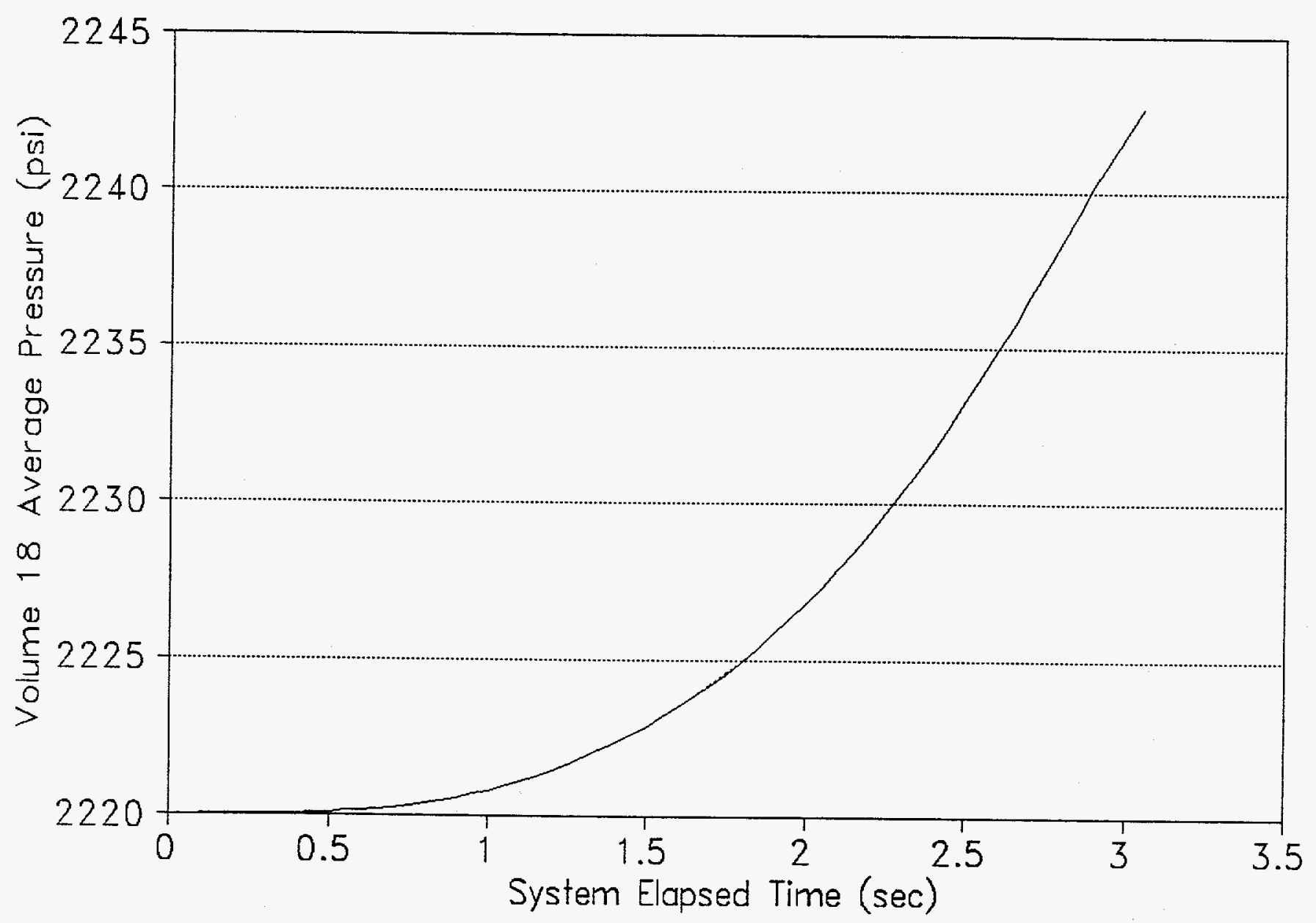


Figure 23

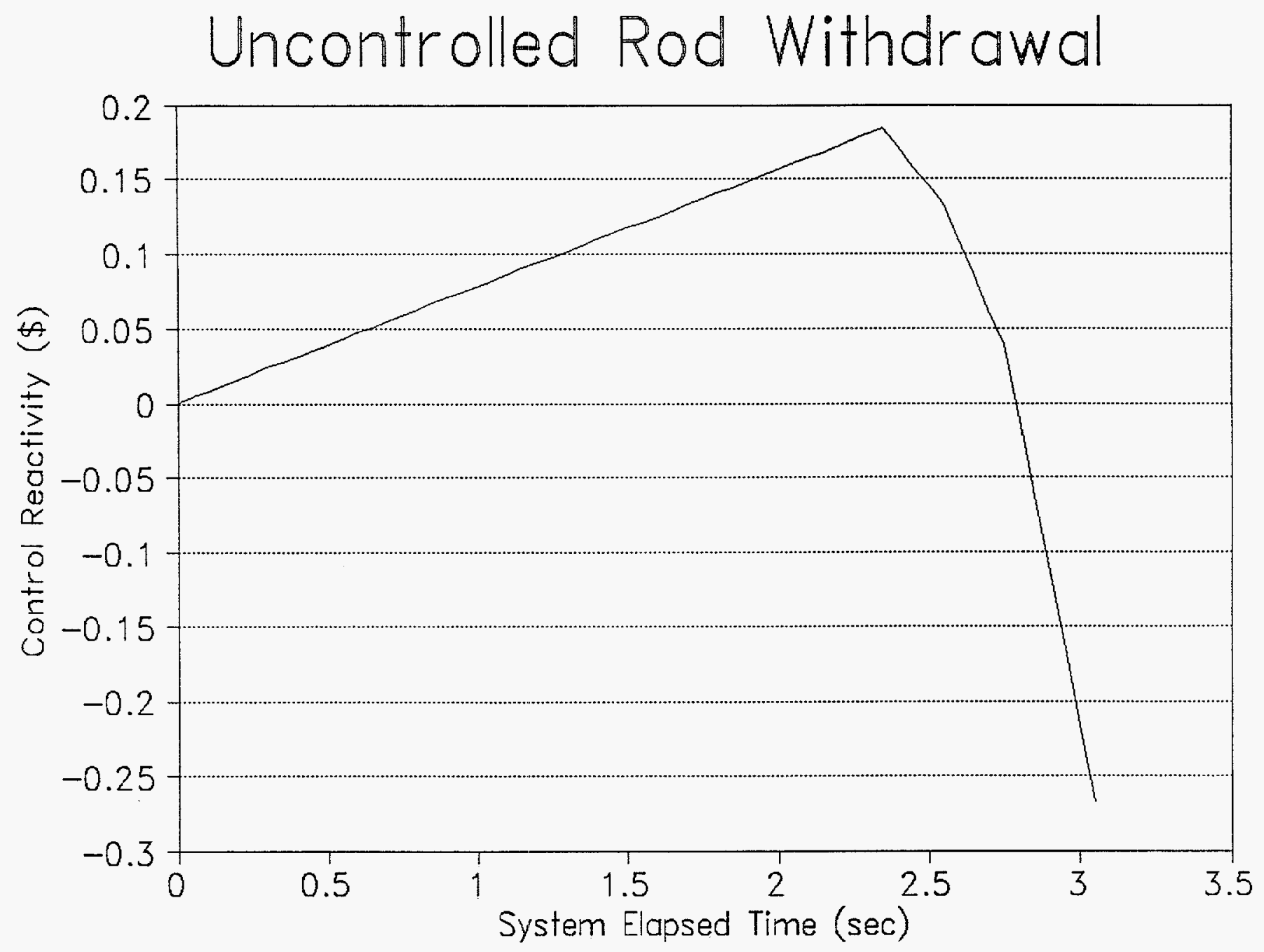


Figure 24

Uncontrolled Rod Withdrawal

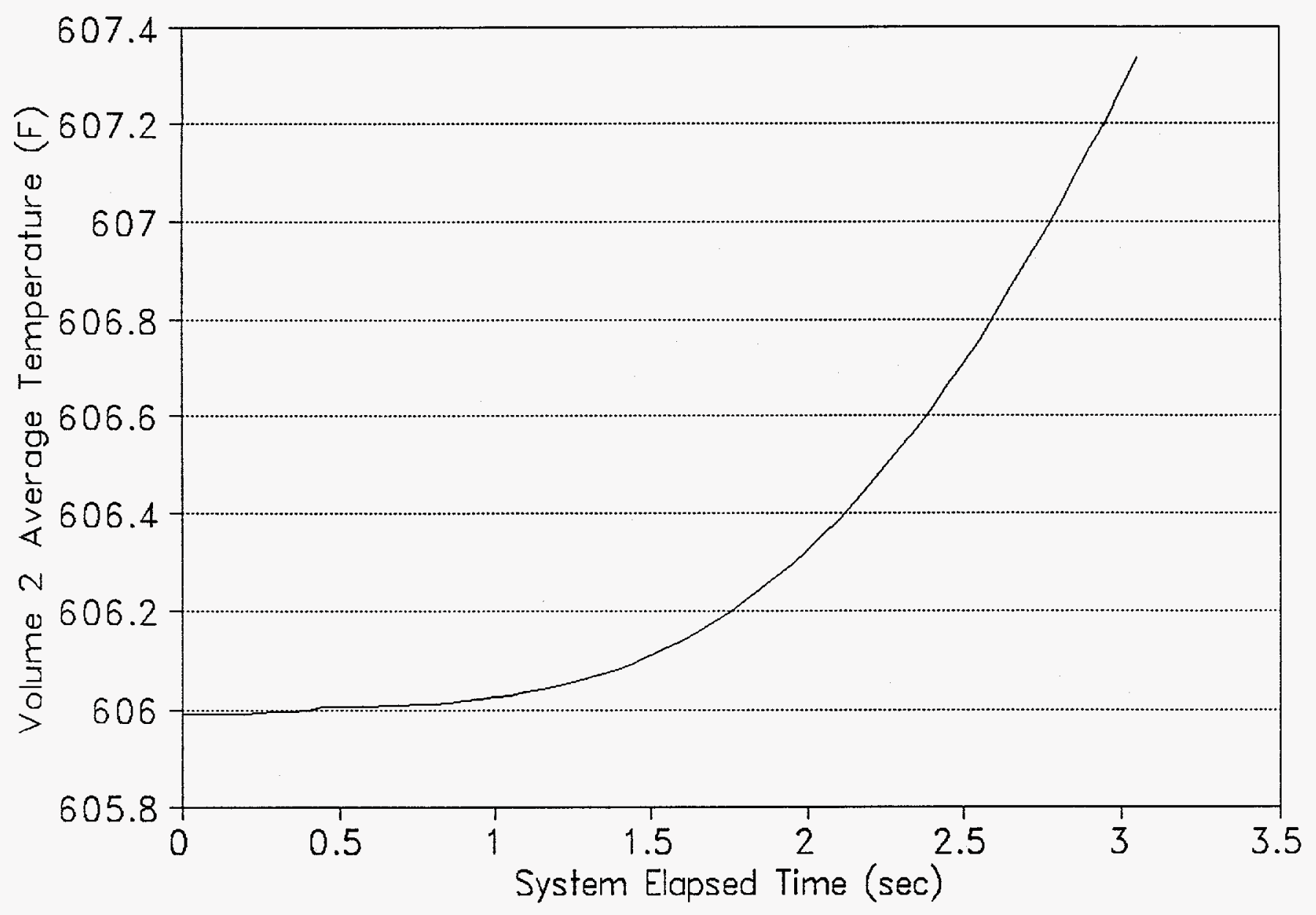


Figure 25

Uncontrolled Rod Withdrawal

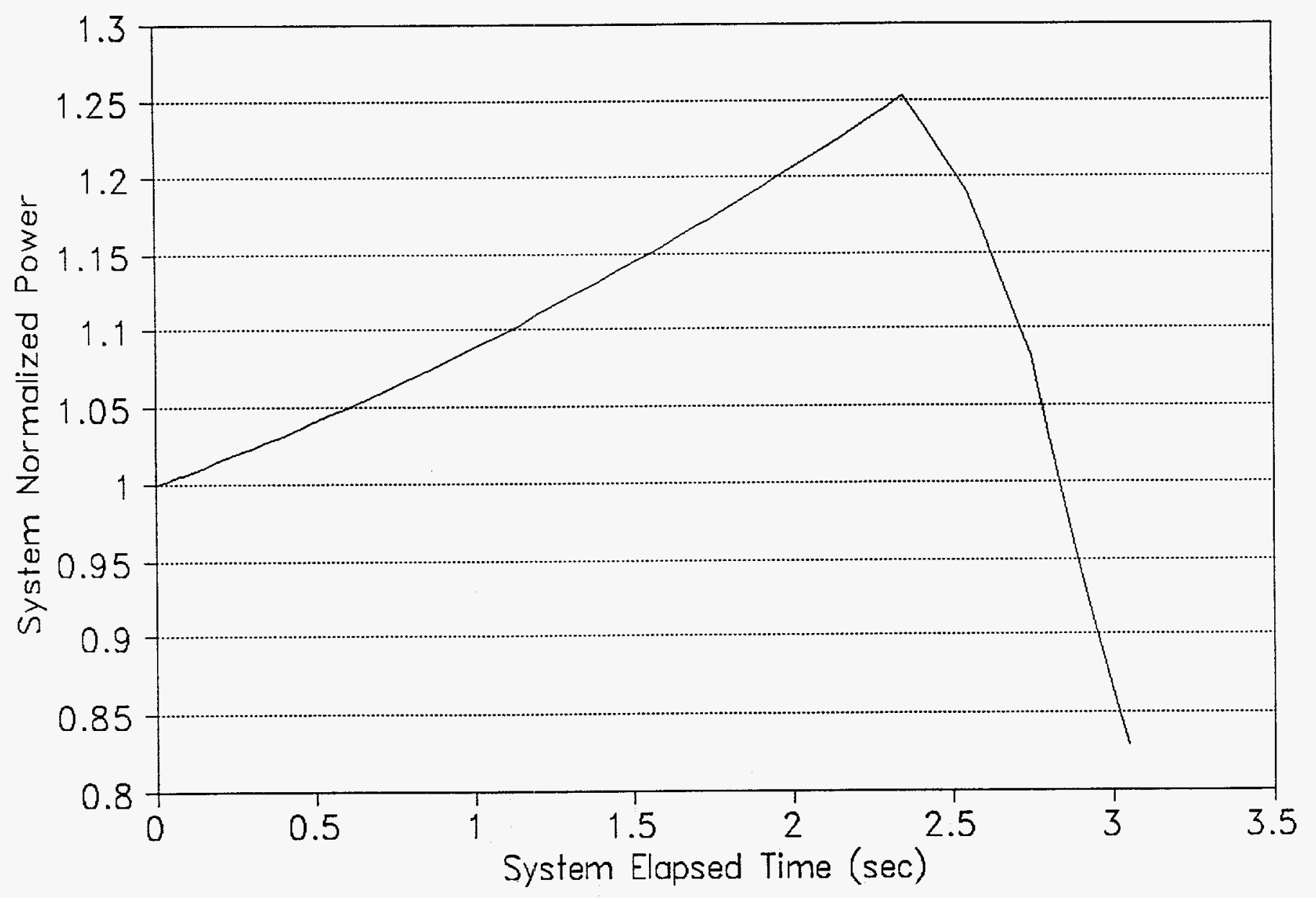


Figure 26

\section{Uncontrolled Rod Withdrawal}

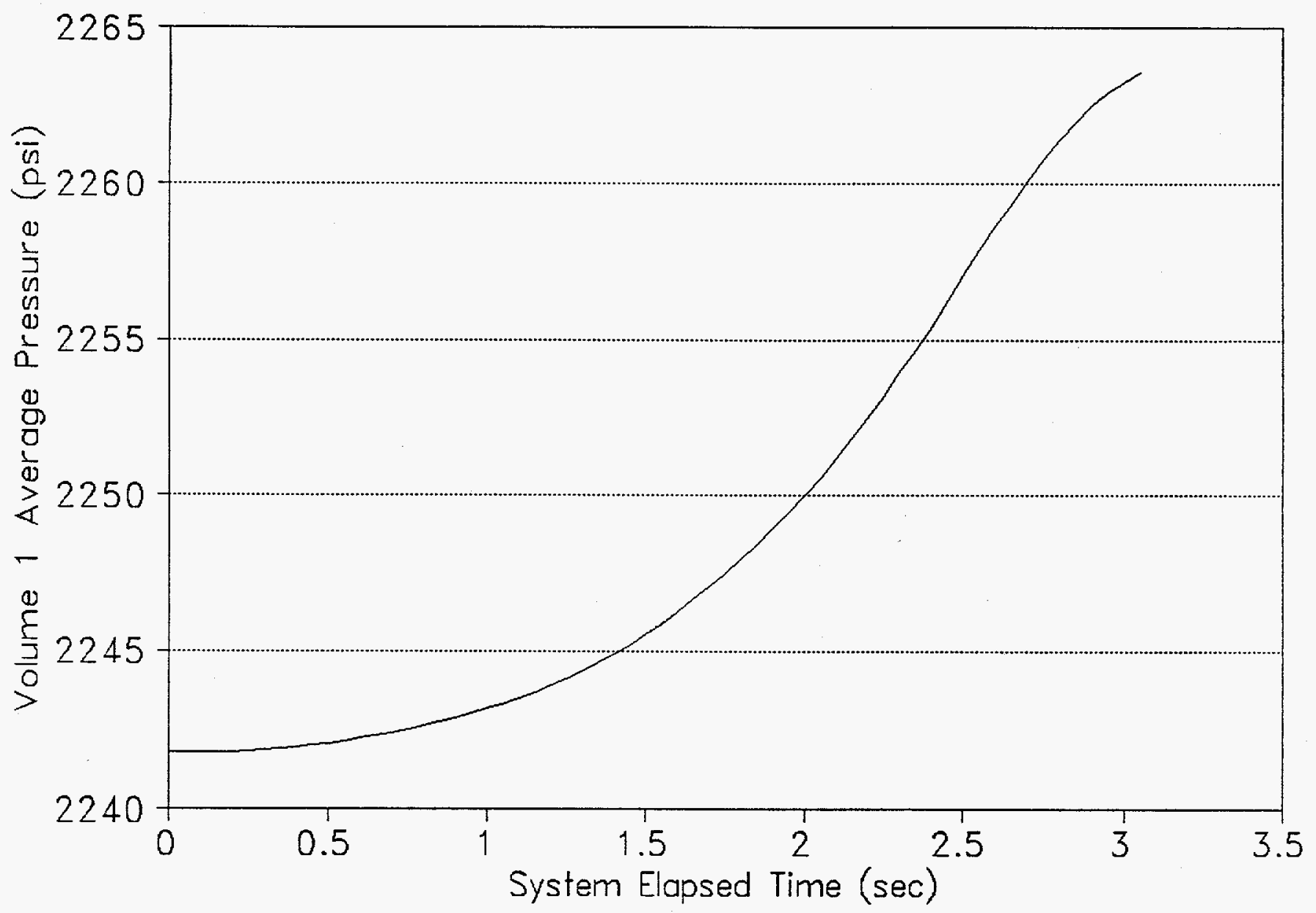


Figure 27

\section{TTWOB With Point Kinetics}

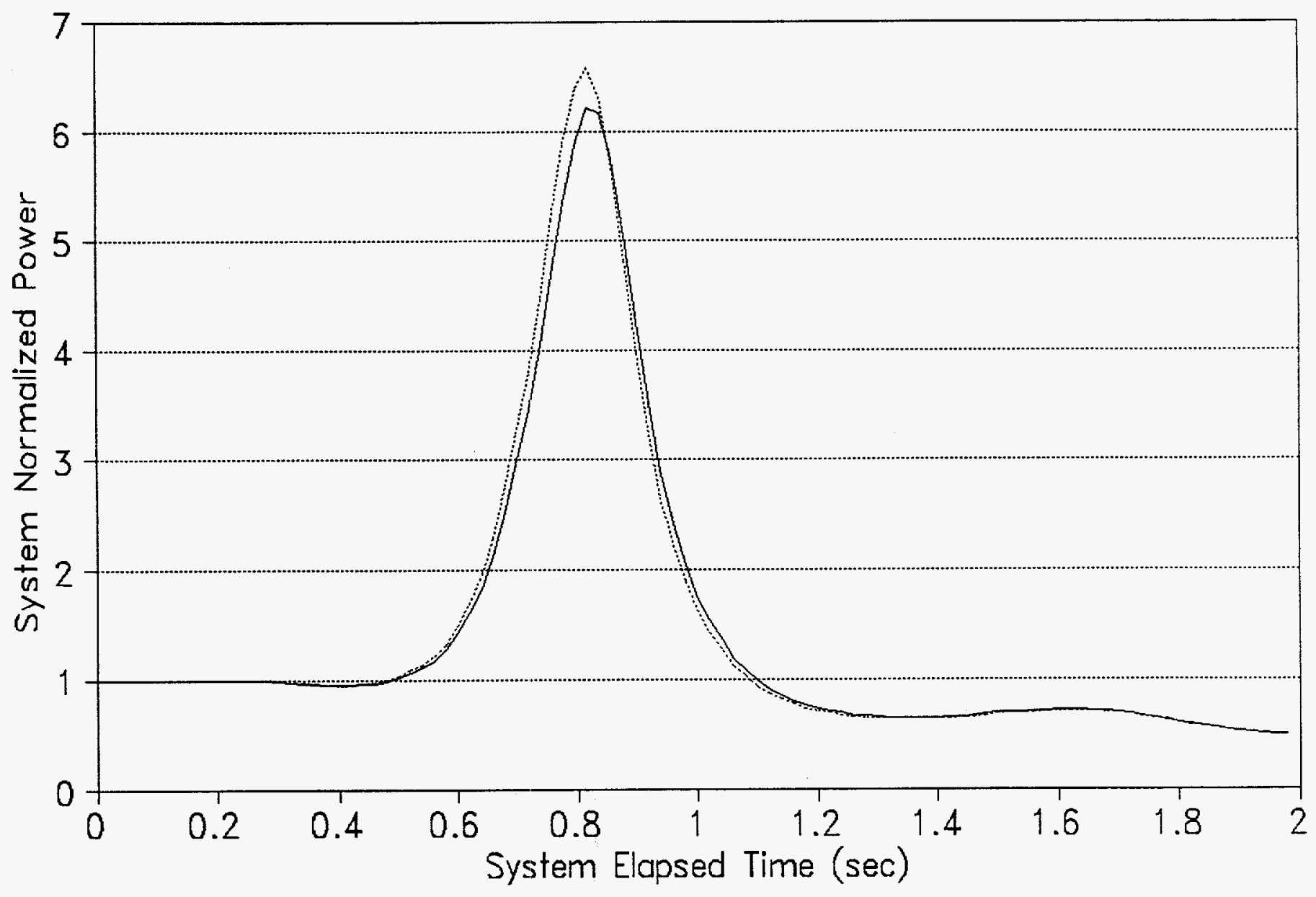


Figure 28

\section{TTWOB With Point Kinetics}

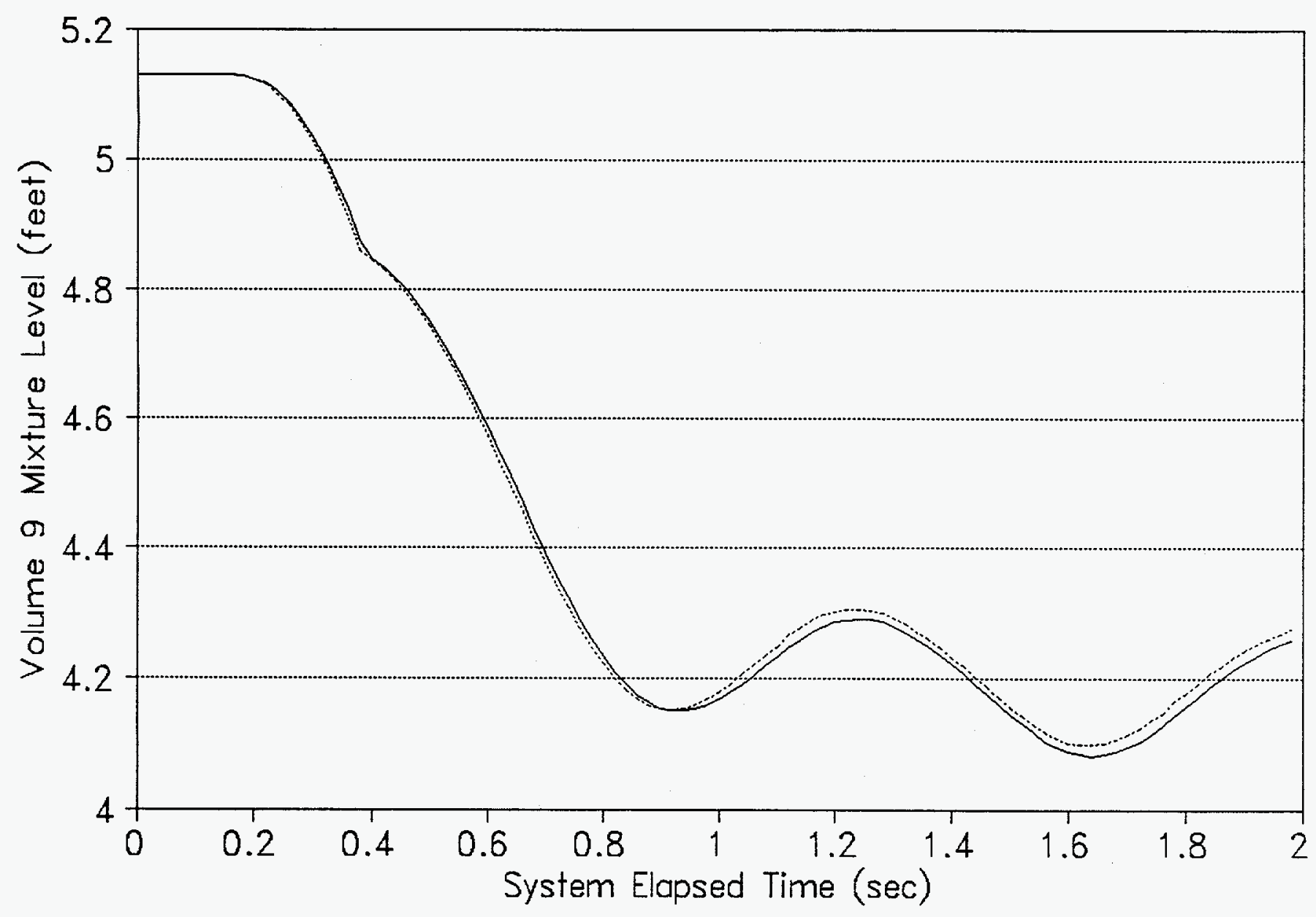


Figure 29

\section{TTWOB With Point Kinetics}

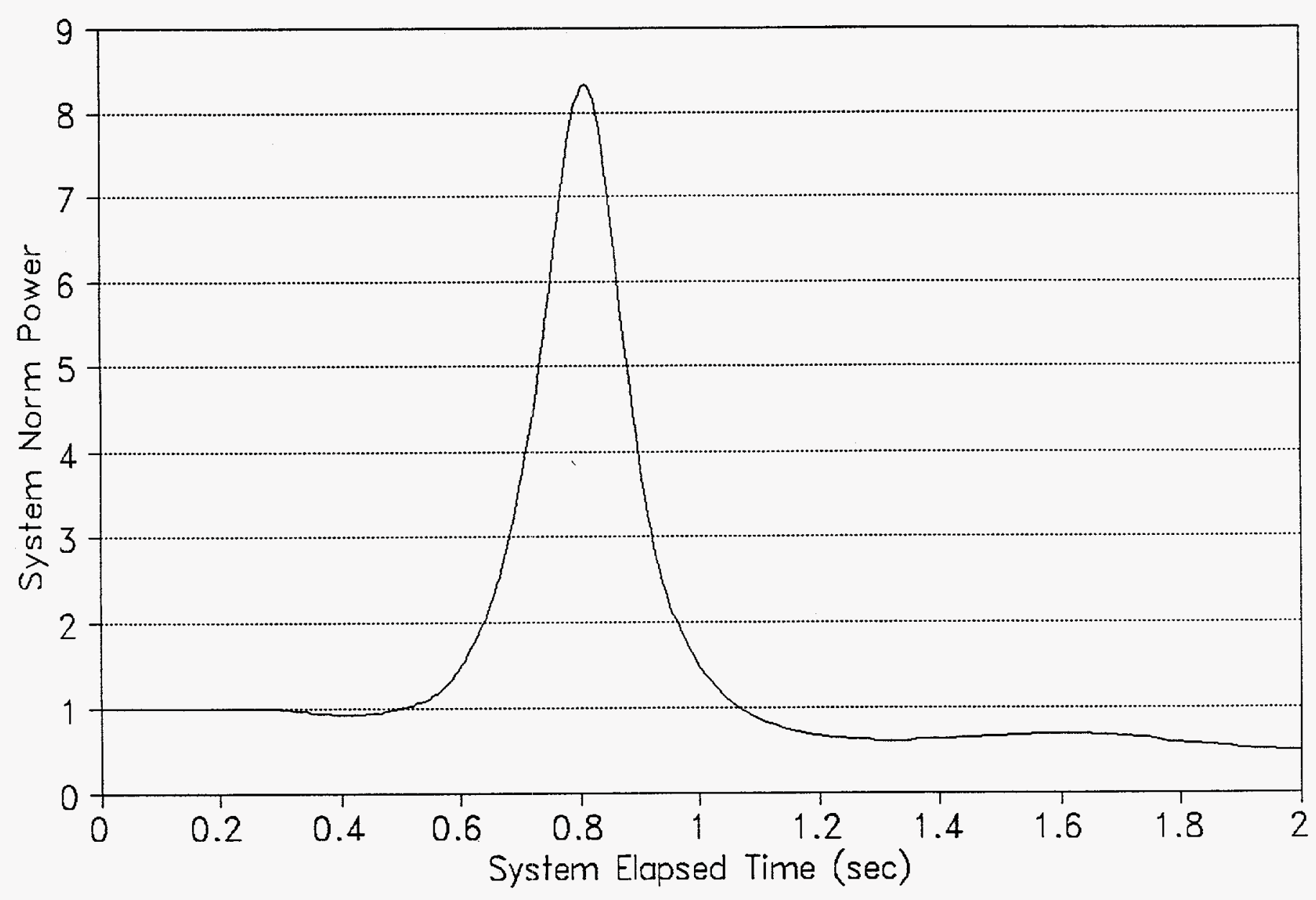


Figure 30

\section{TTWOB With Point Kinetics}

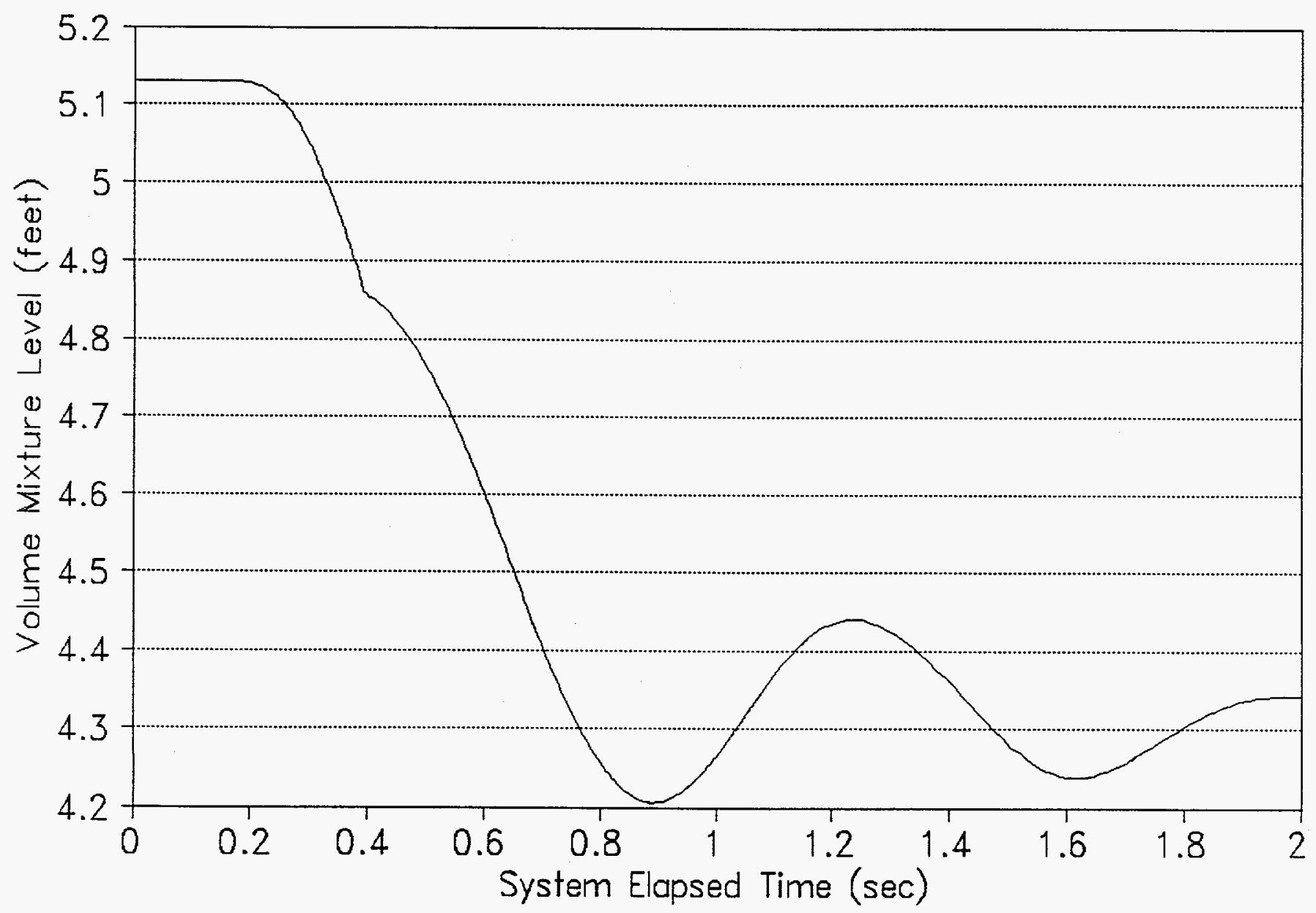


Figure 31

\section{TTWOB With Point Kinetics}

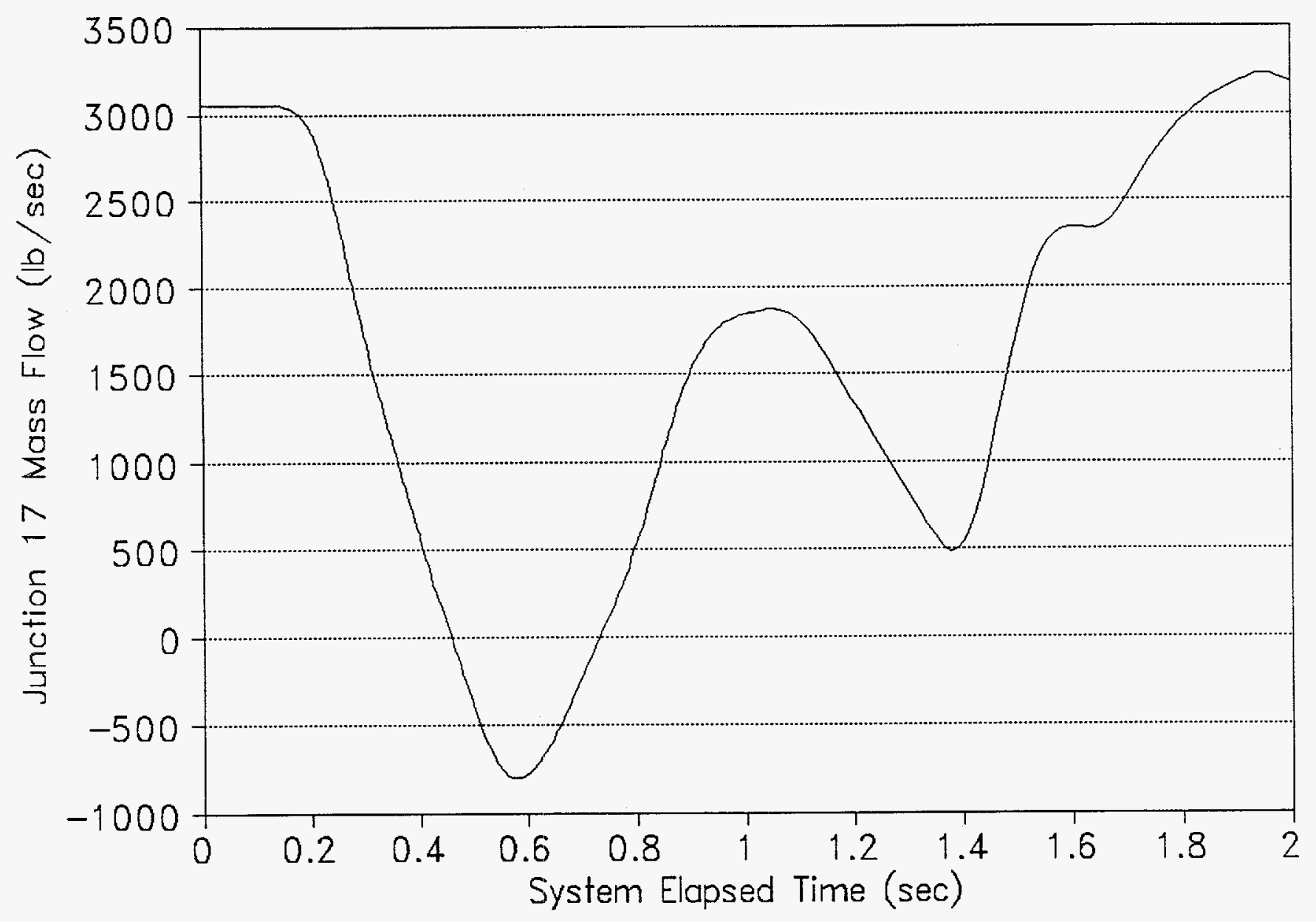


Figure 32

TTWOB With Point Kinetics

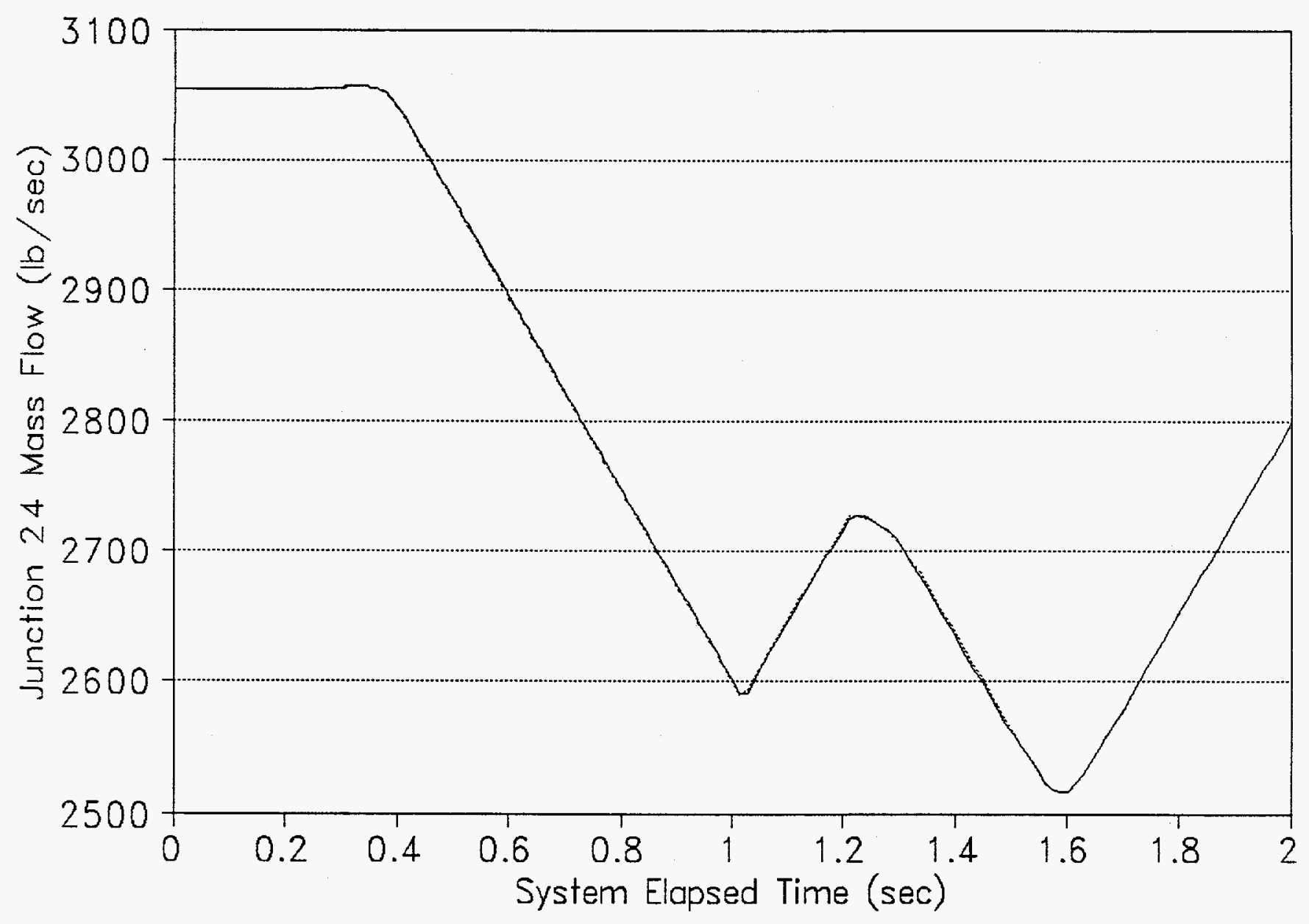


Figure 33

\section{TTWOB With Point Kinetics}

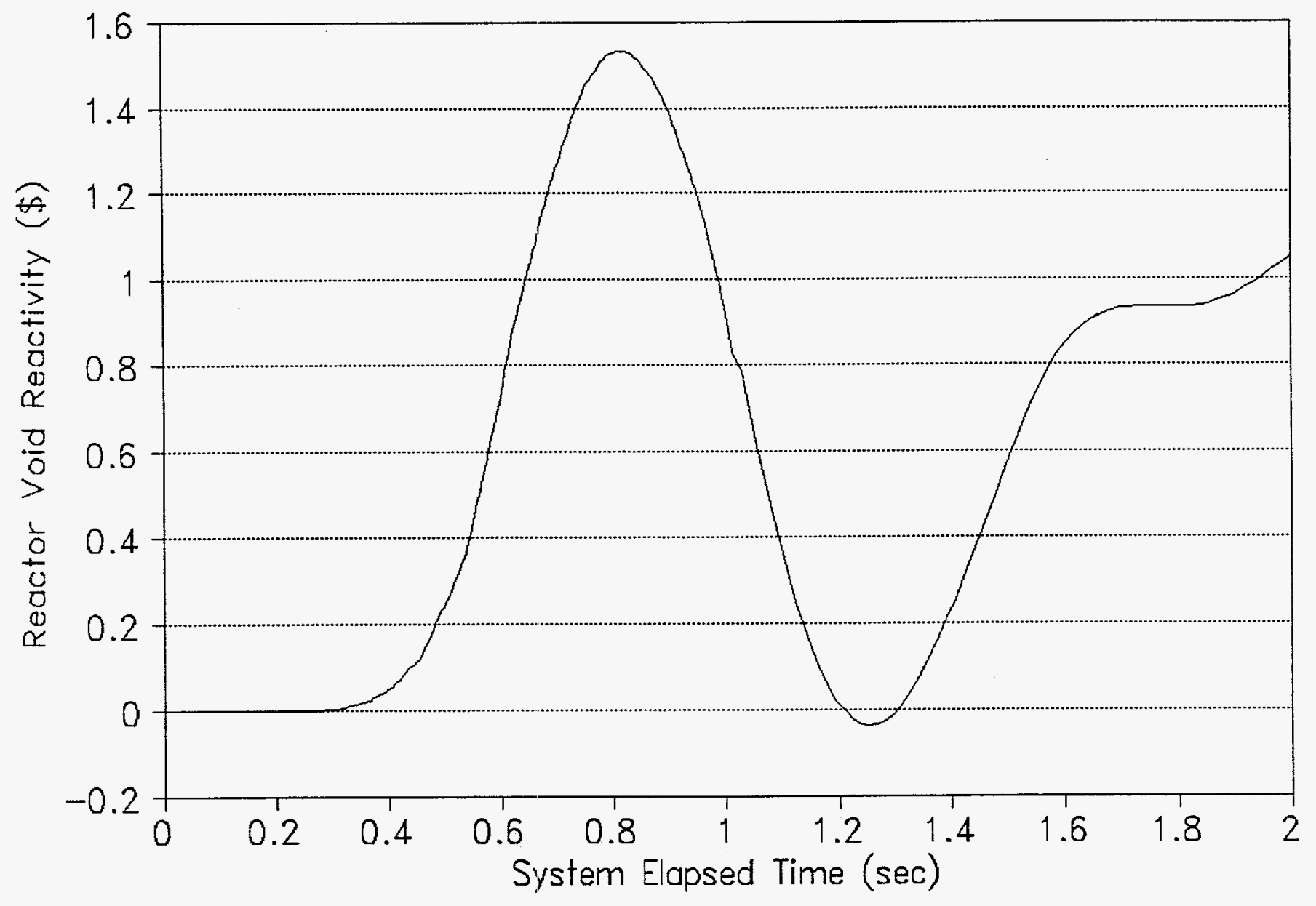


Figure 34

\section{TTWOB With Point Kinetics}

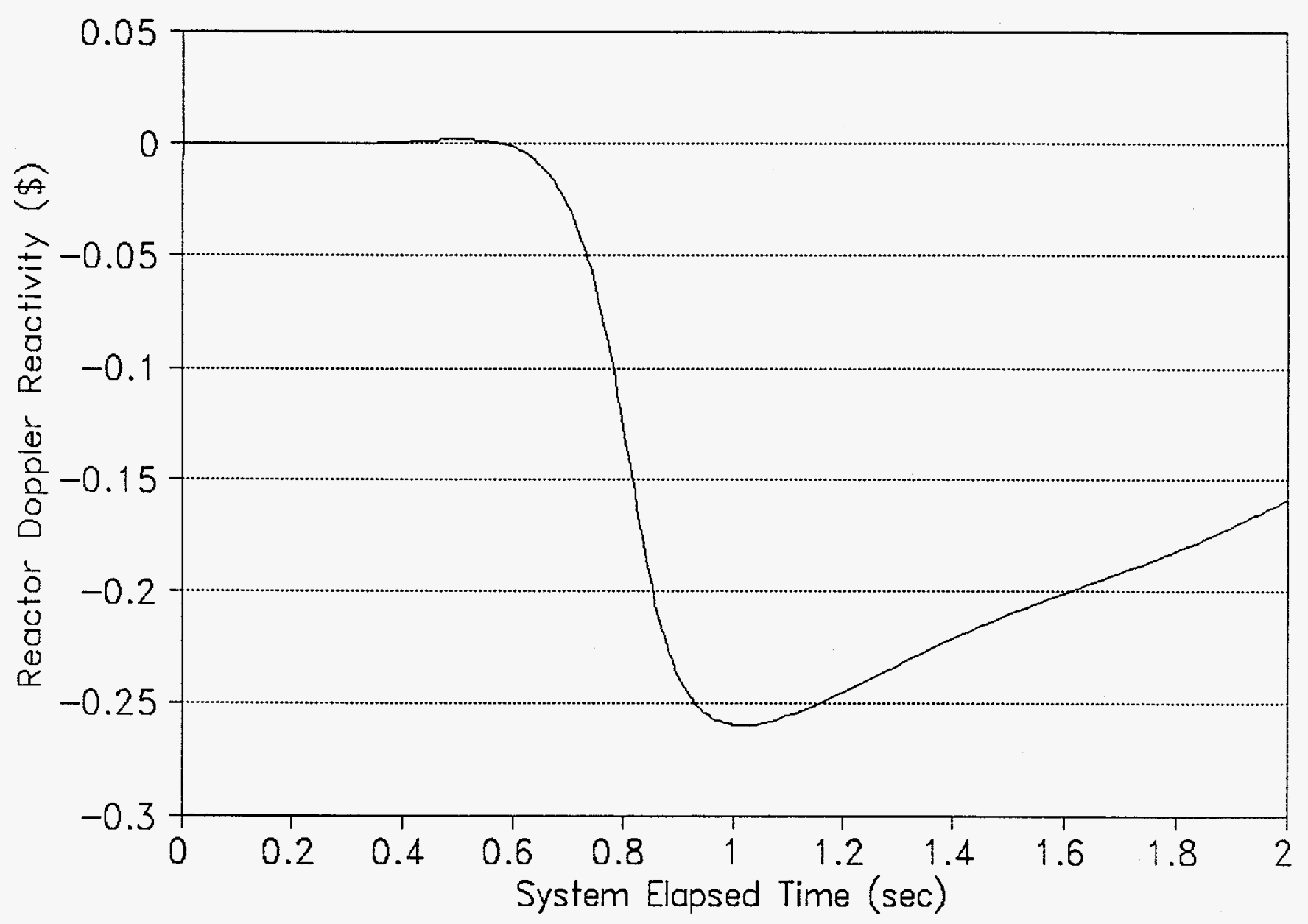


Figure 35

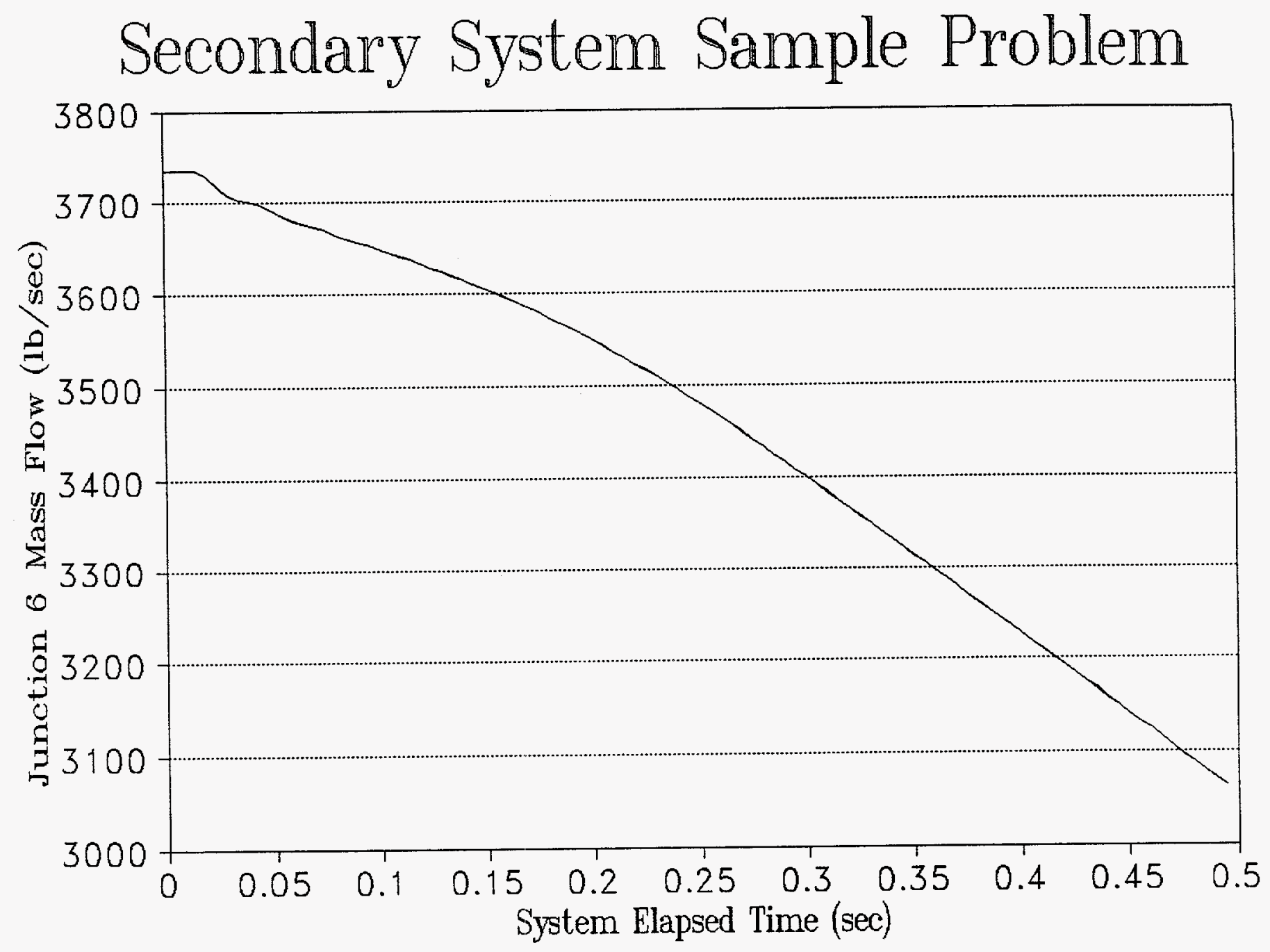


Figure 36

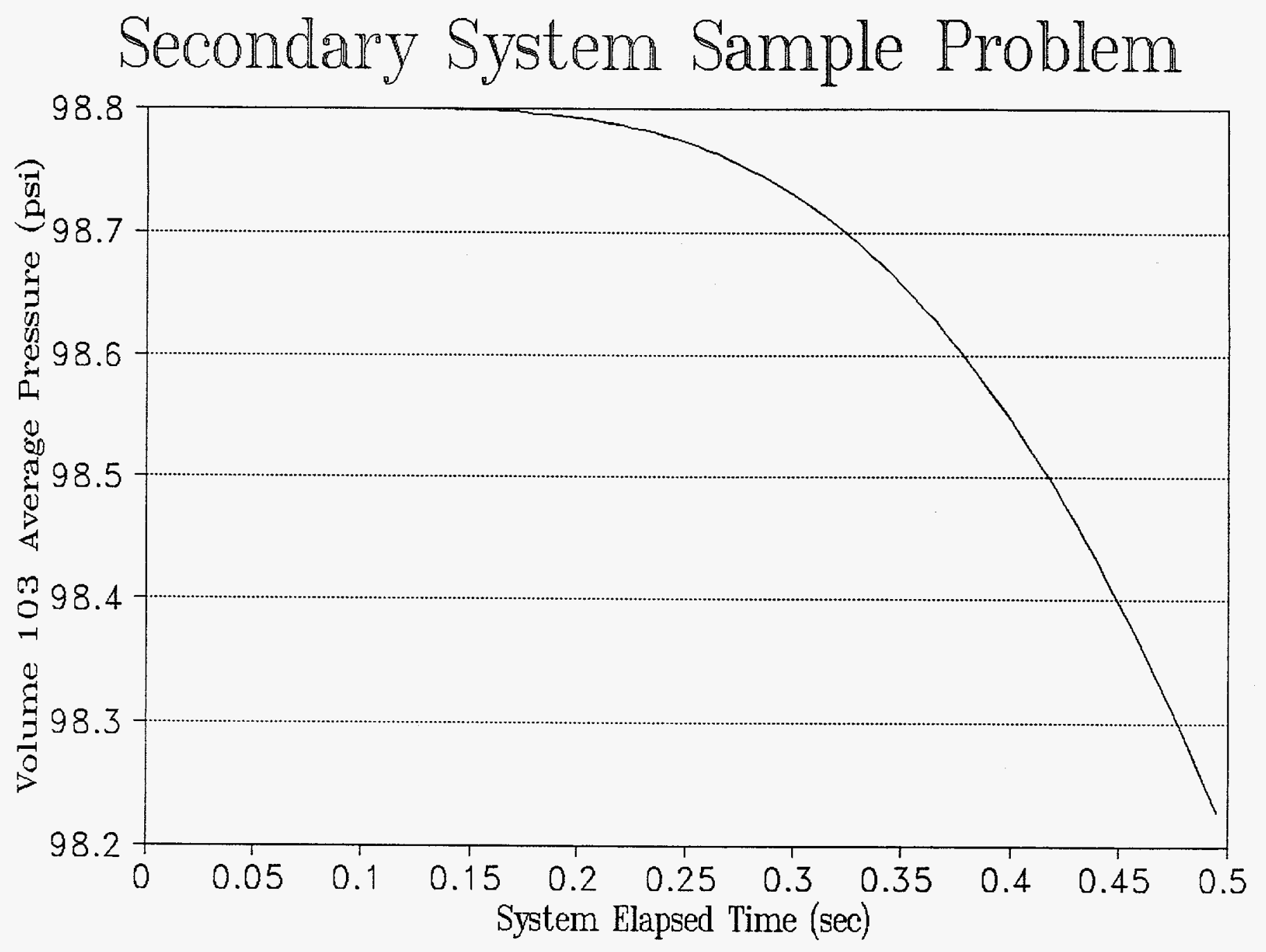


Figure 37

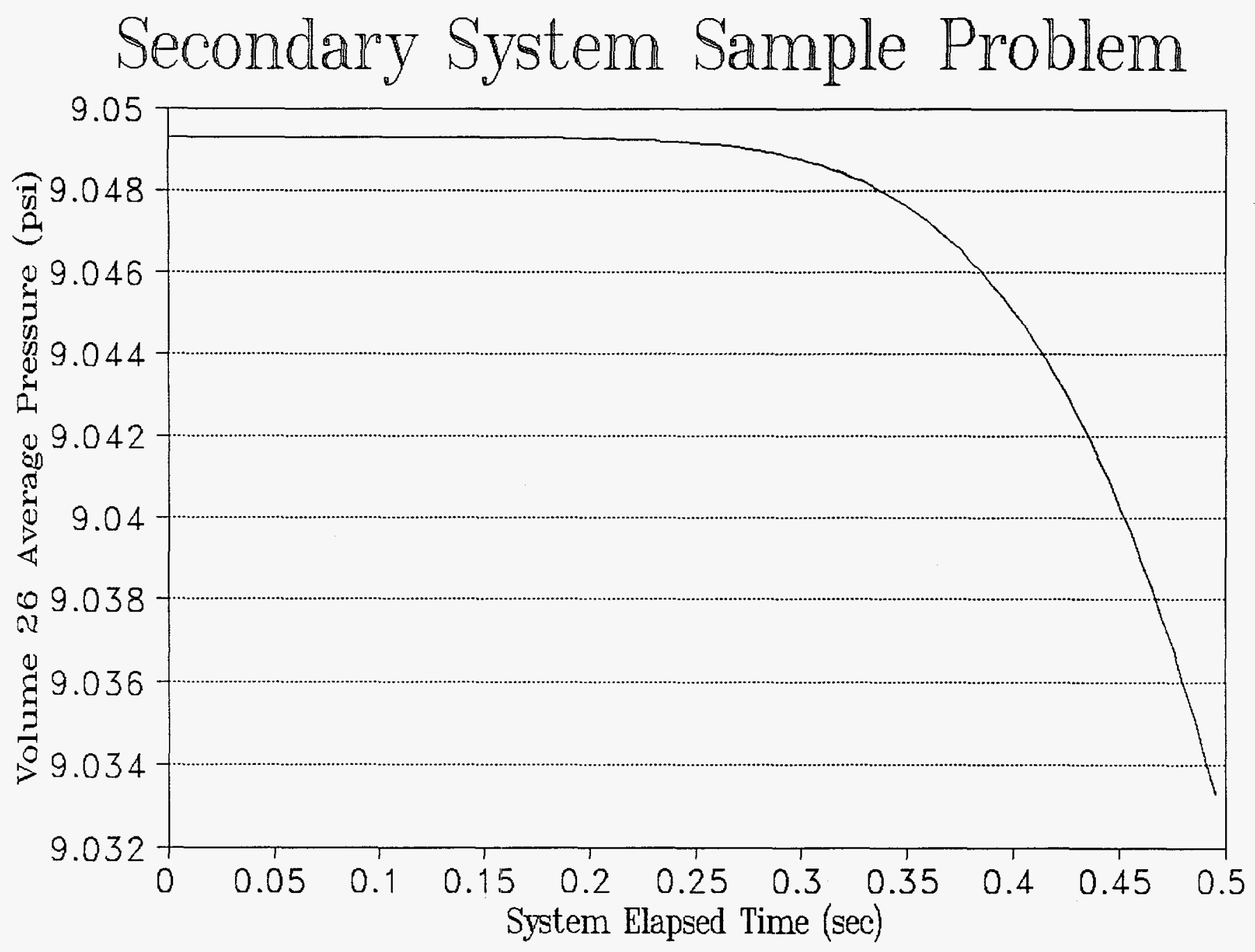


Figure 38

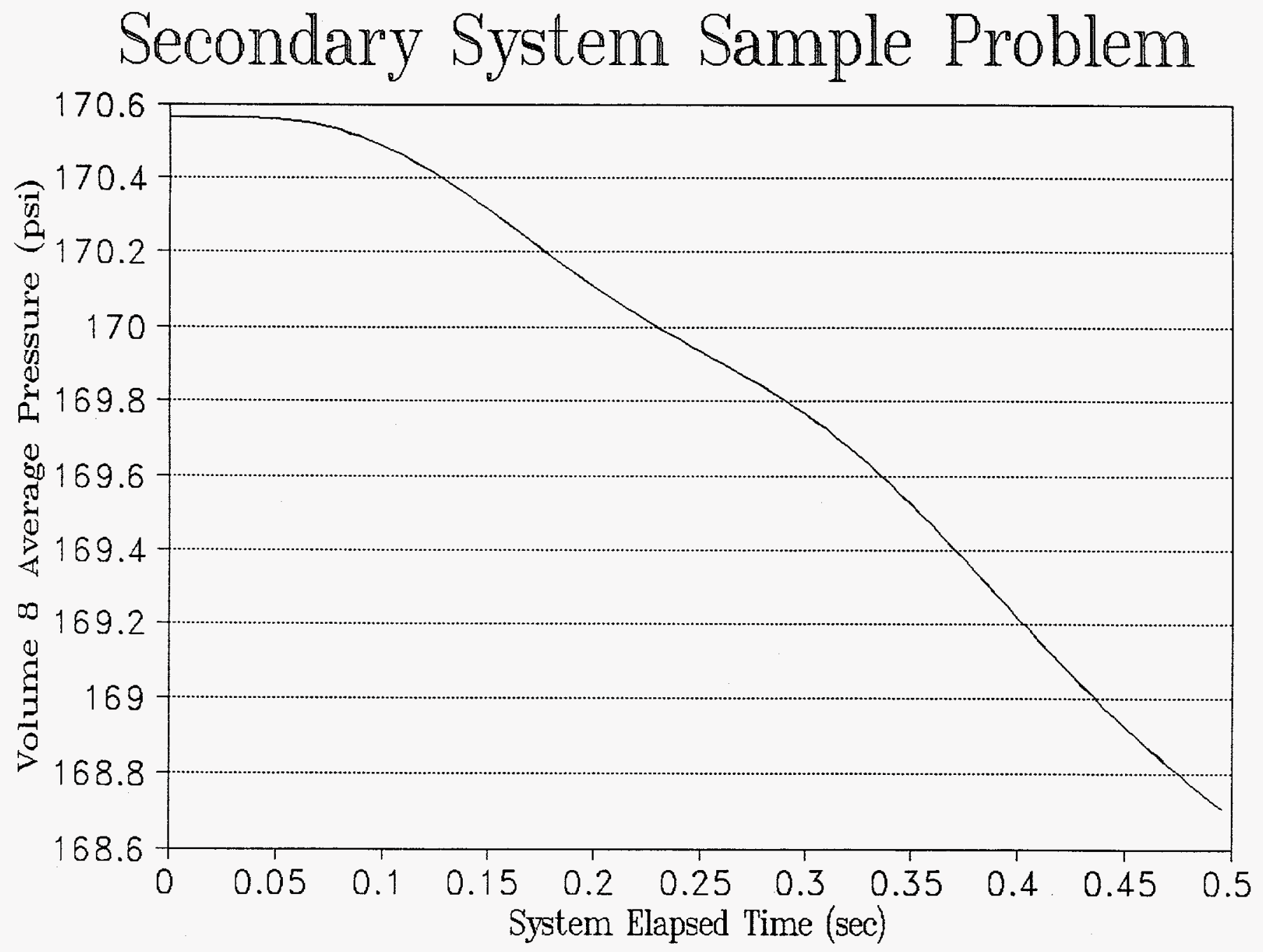


Figure 39

\section{Secondary System Sample Problem}

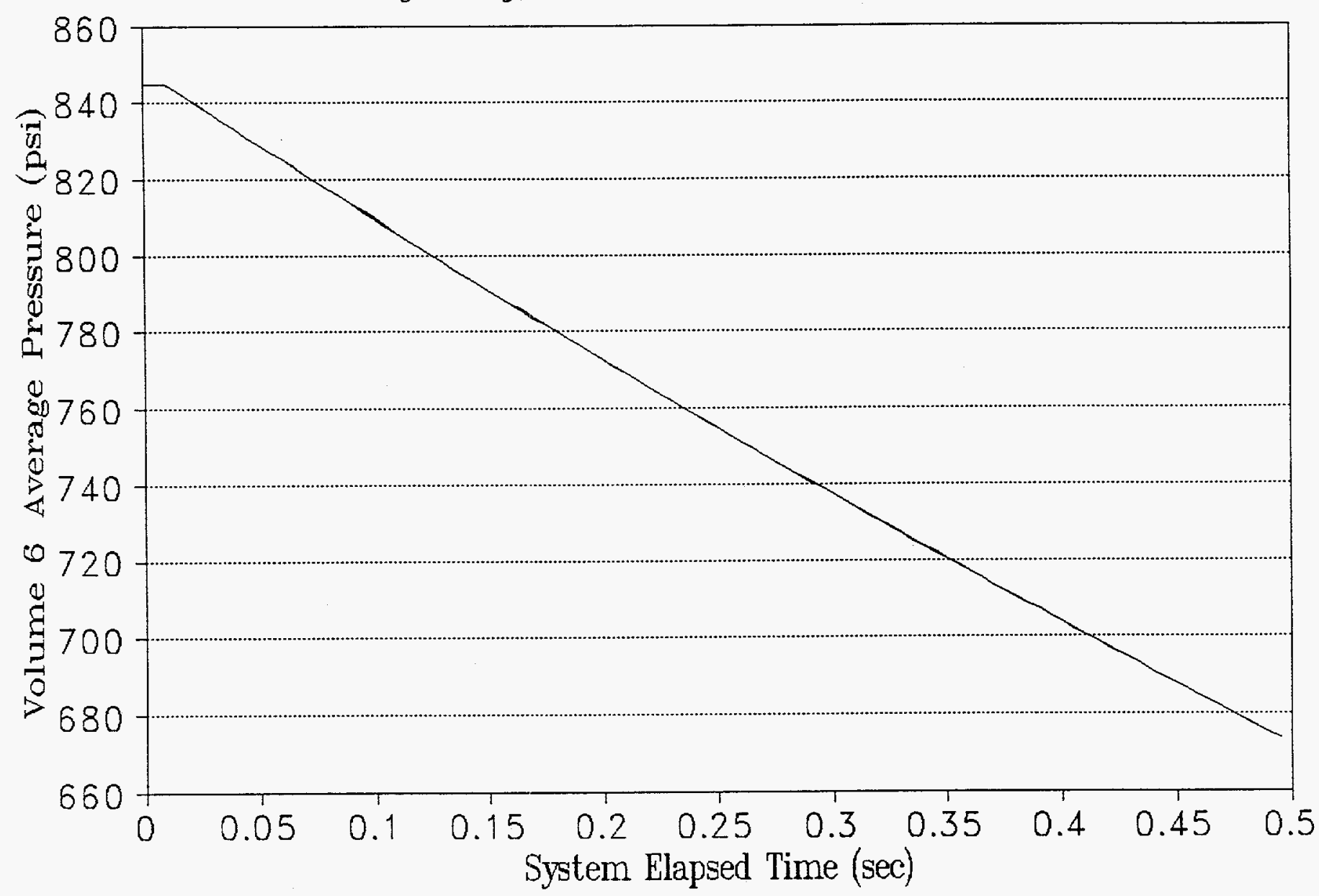


Figure 40

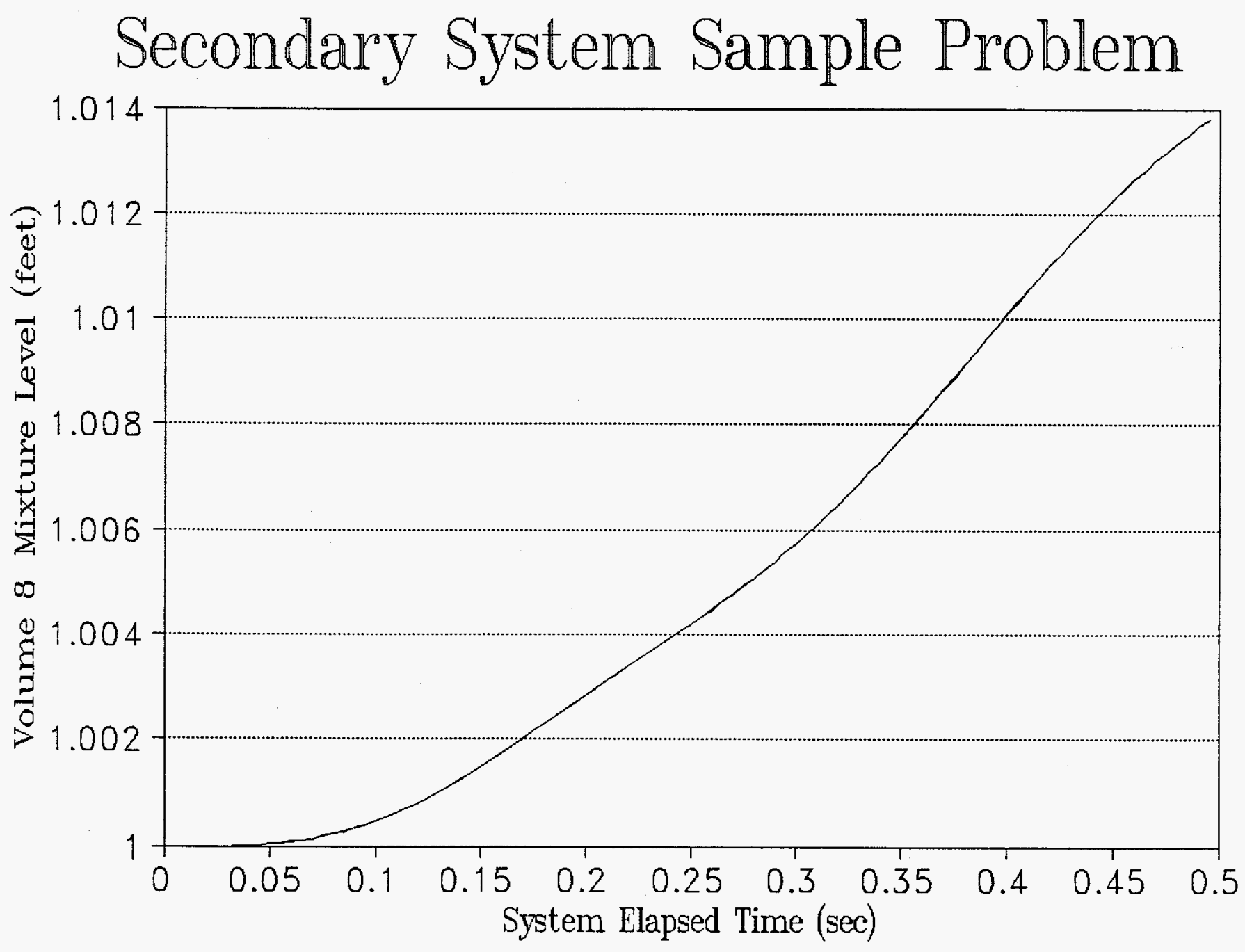


Figure 41

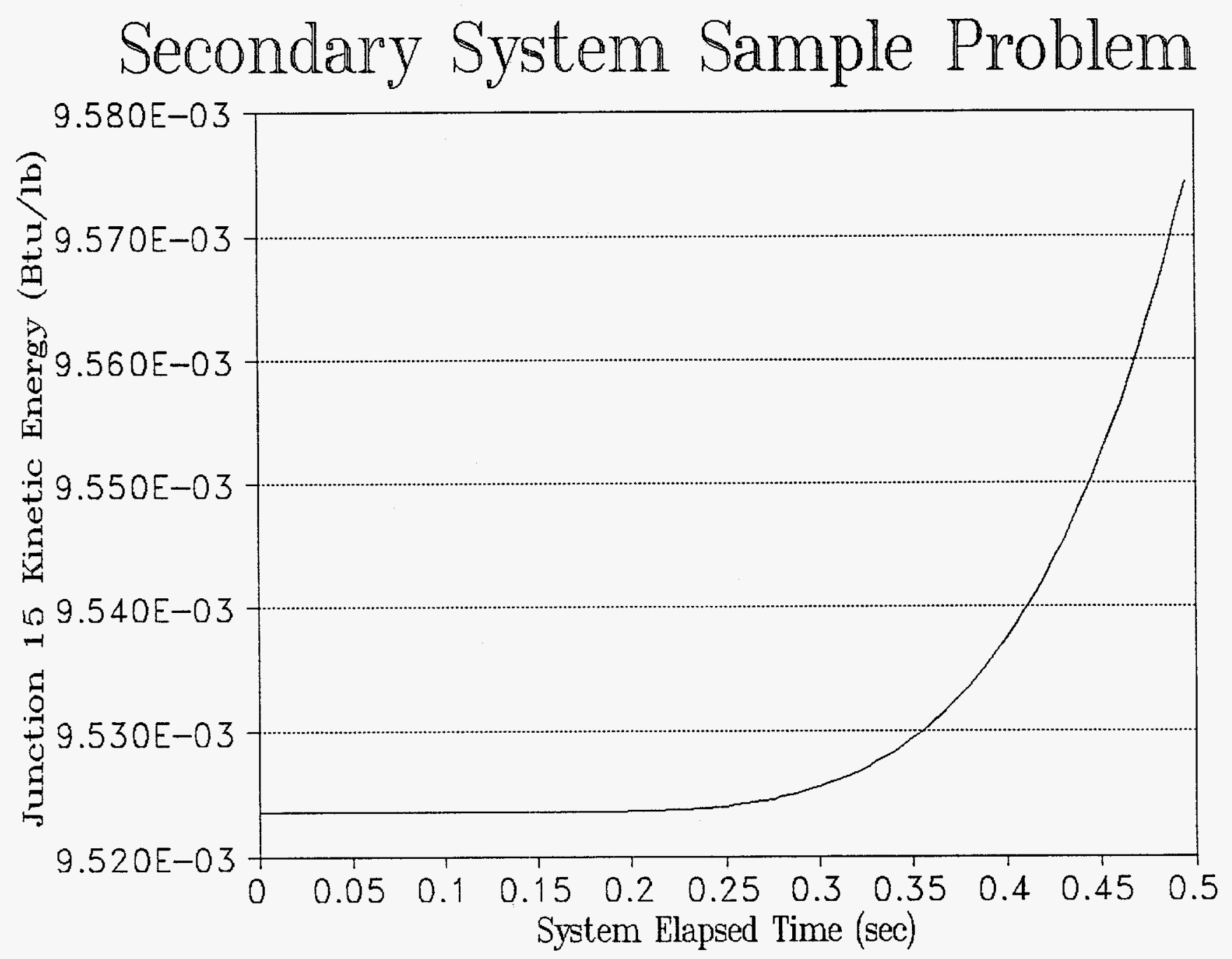


Figure 42

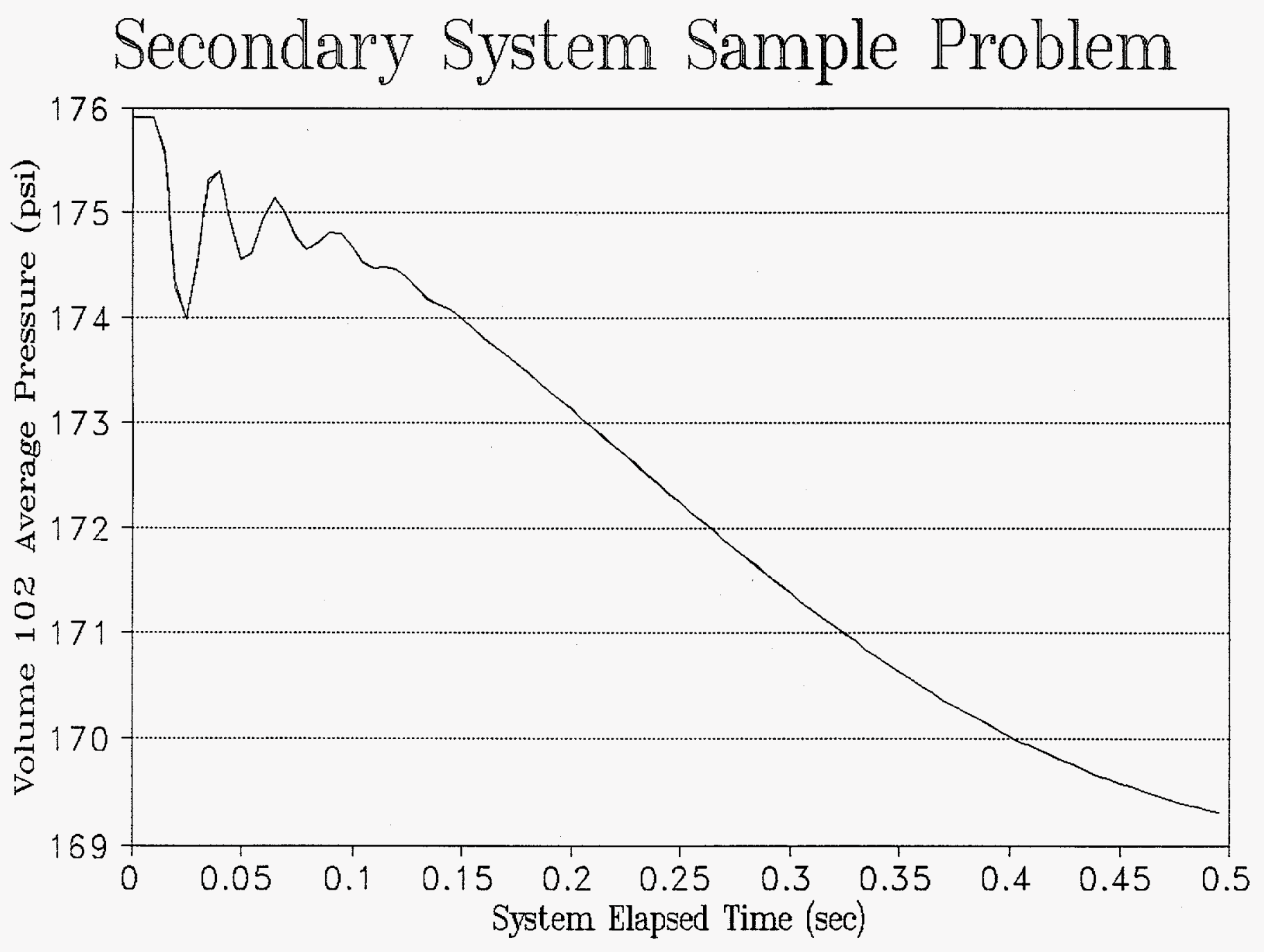


Figure 43

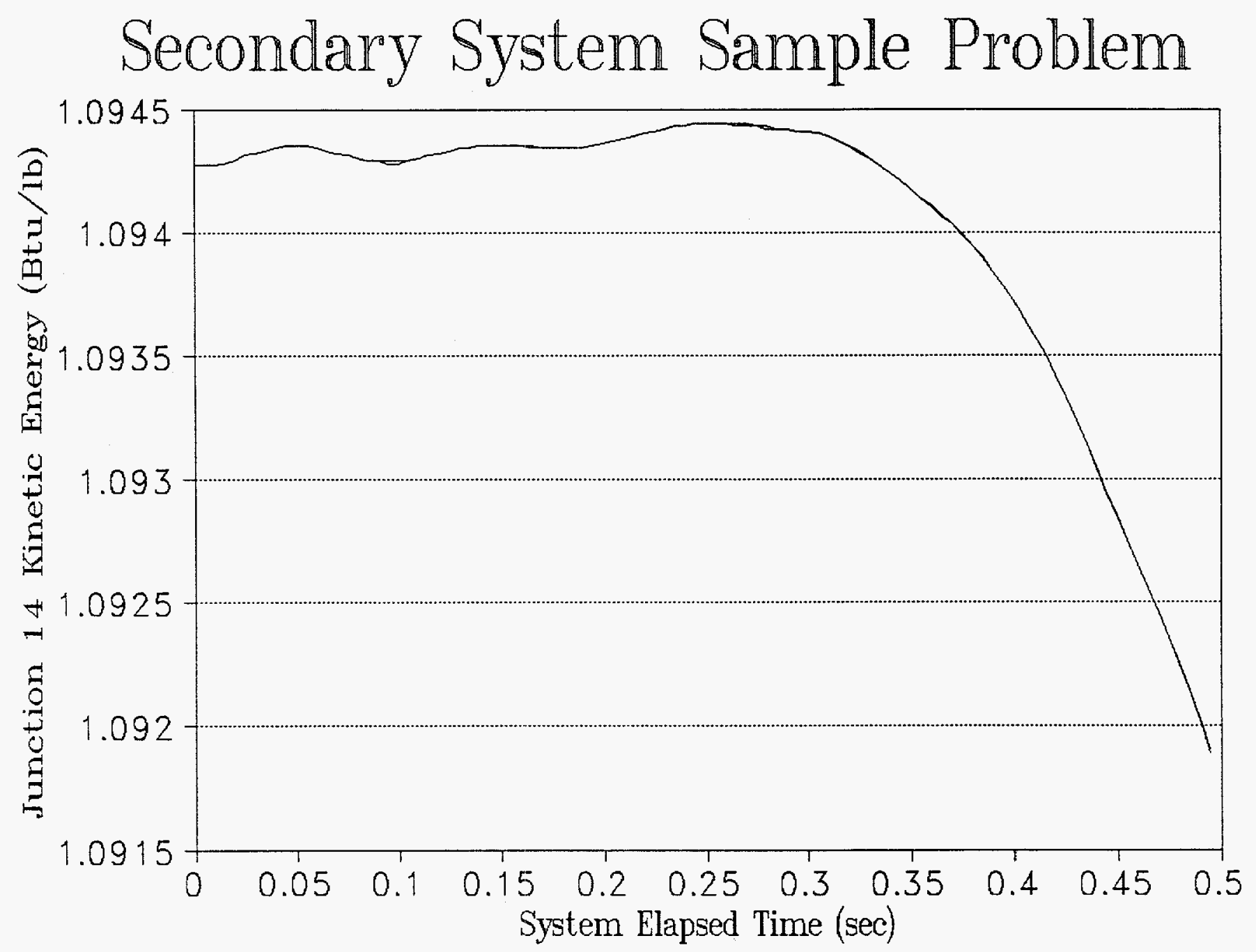


Figure 44

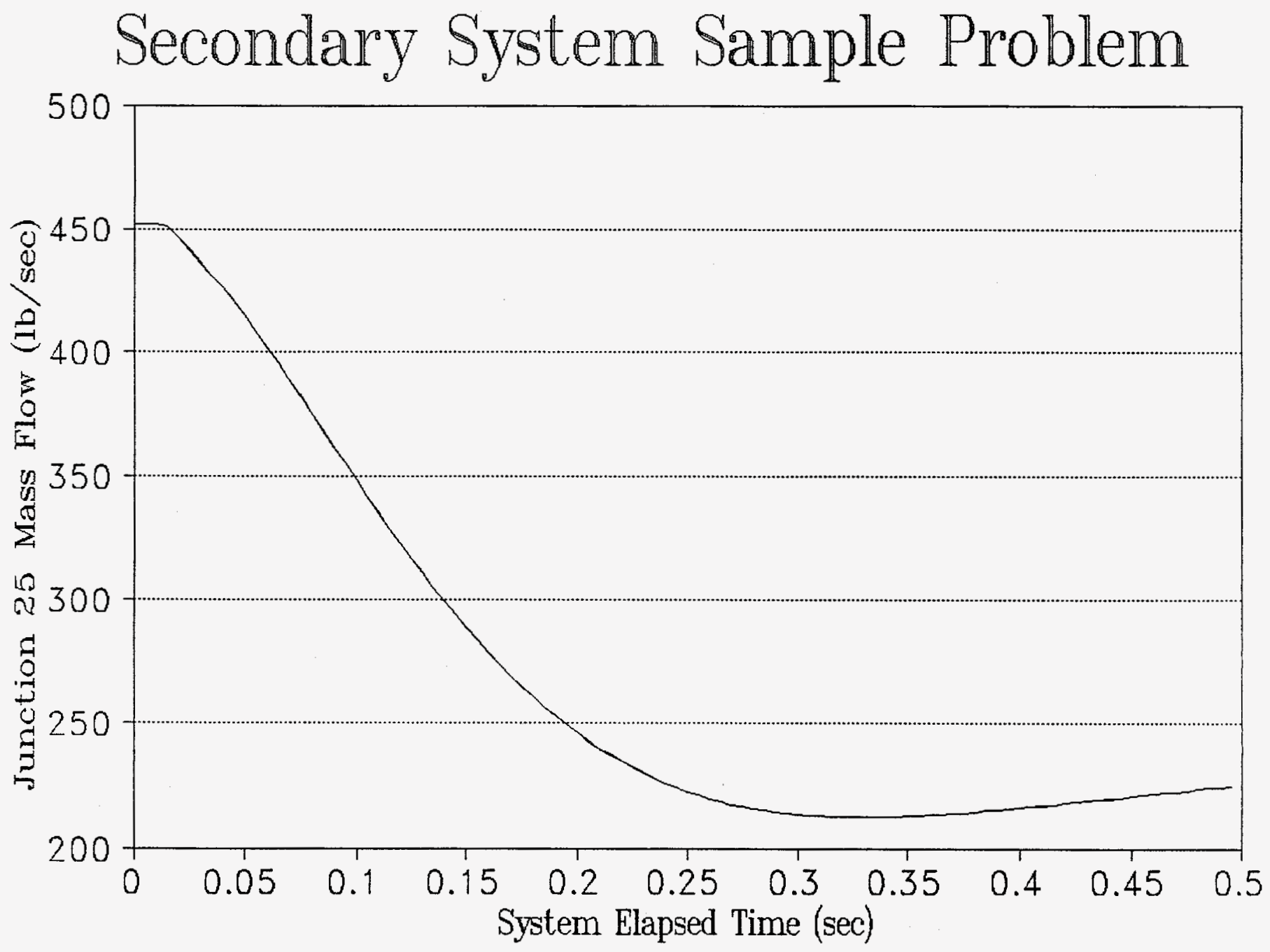


Figure 45

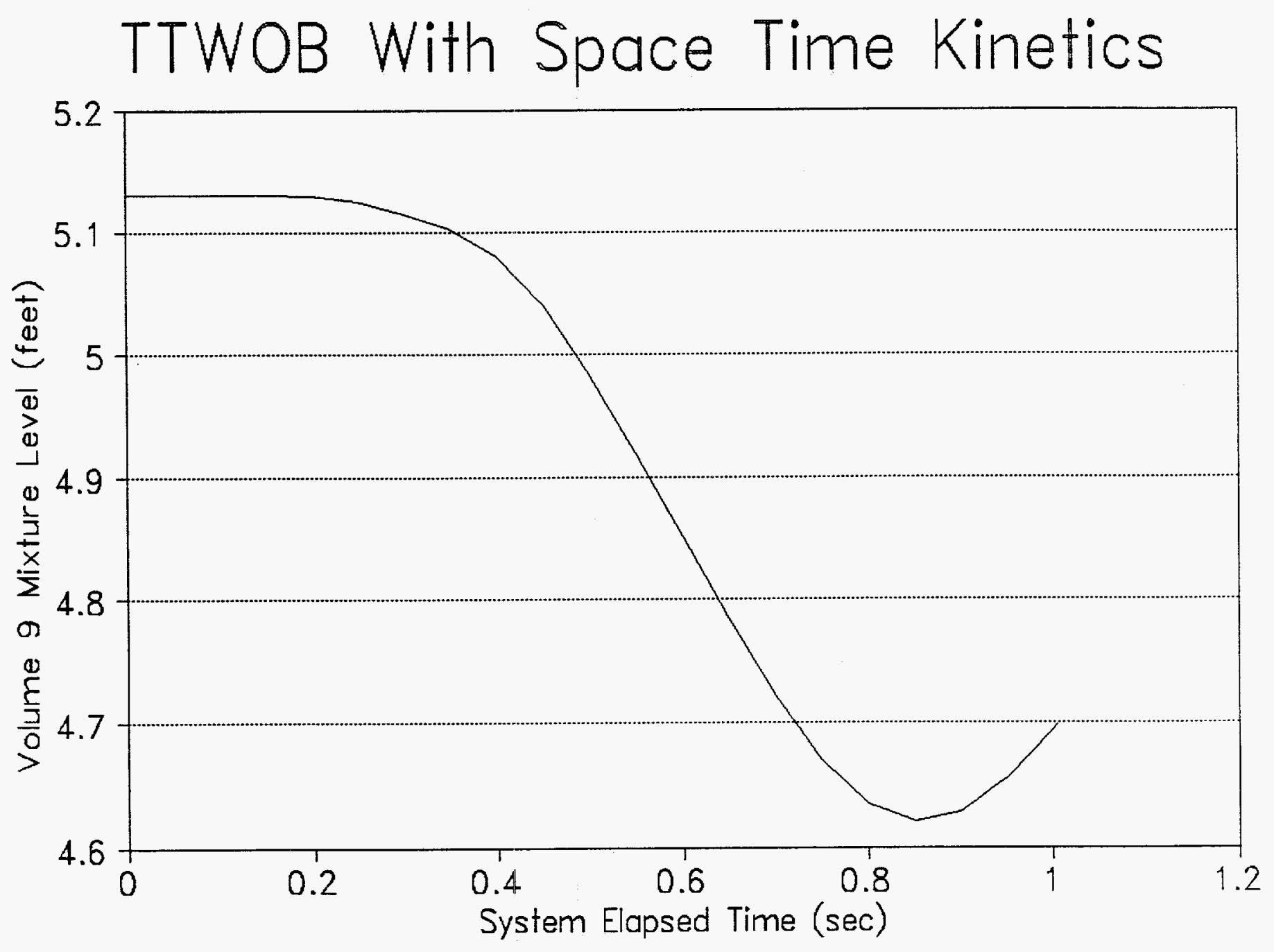


Figure 46

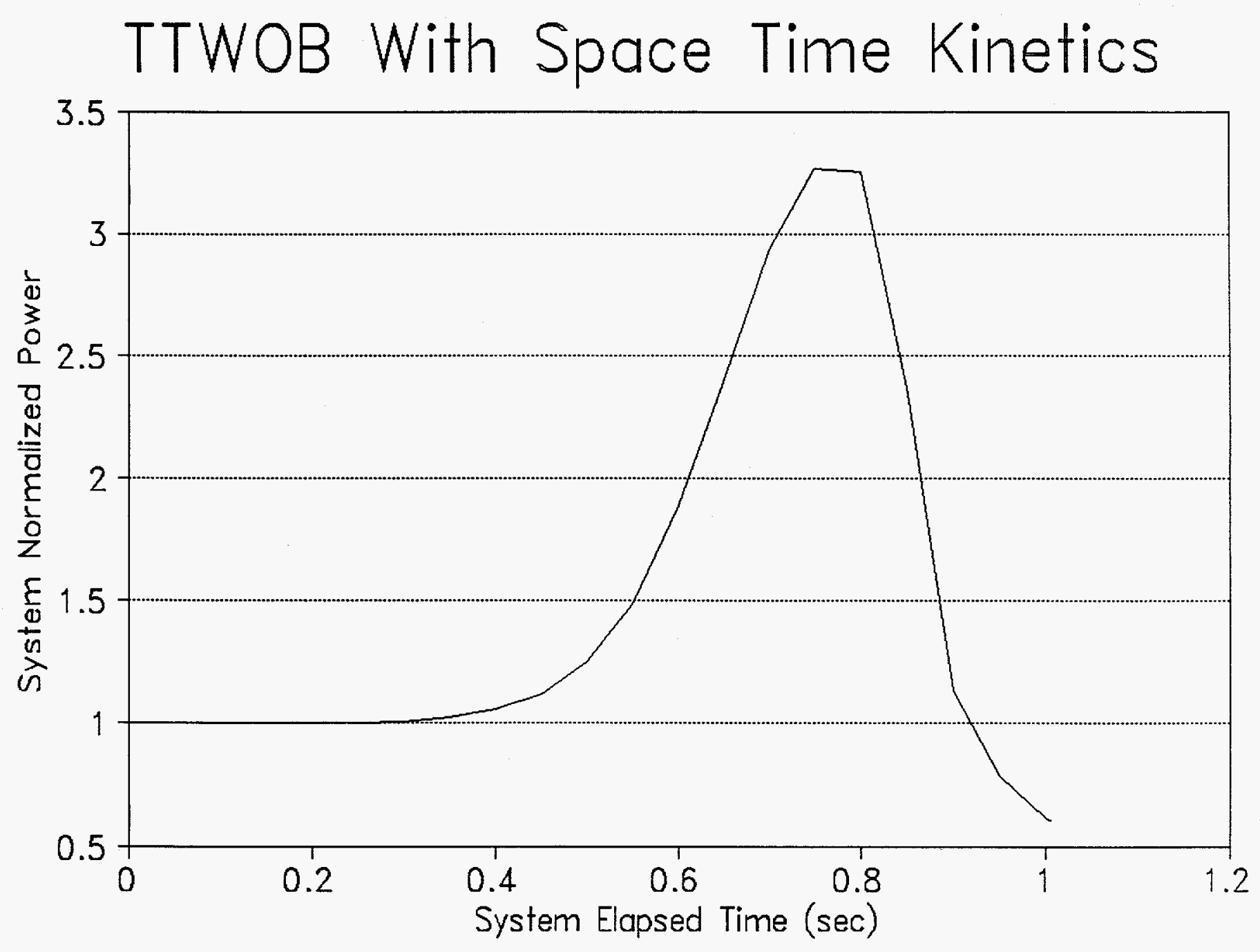


Figure 47

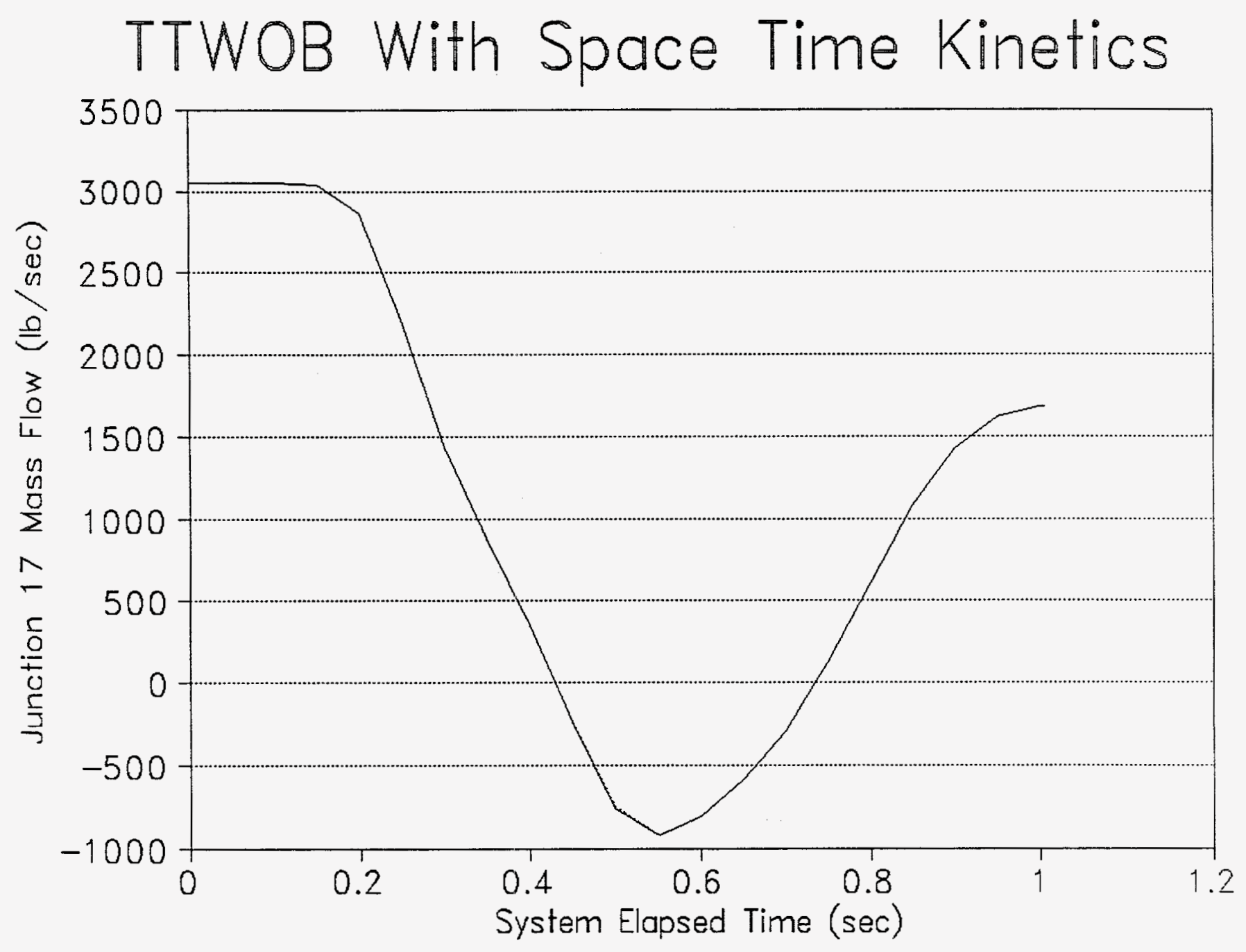


Figure 48

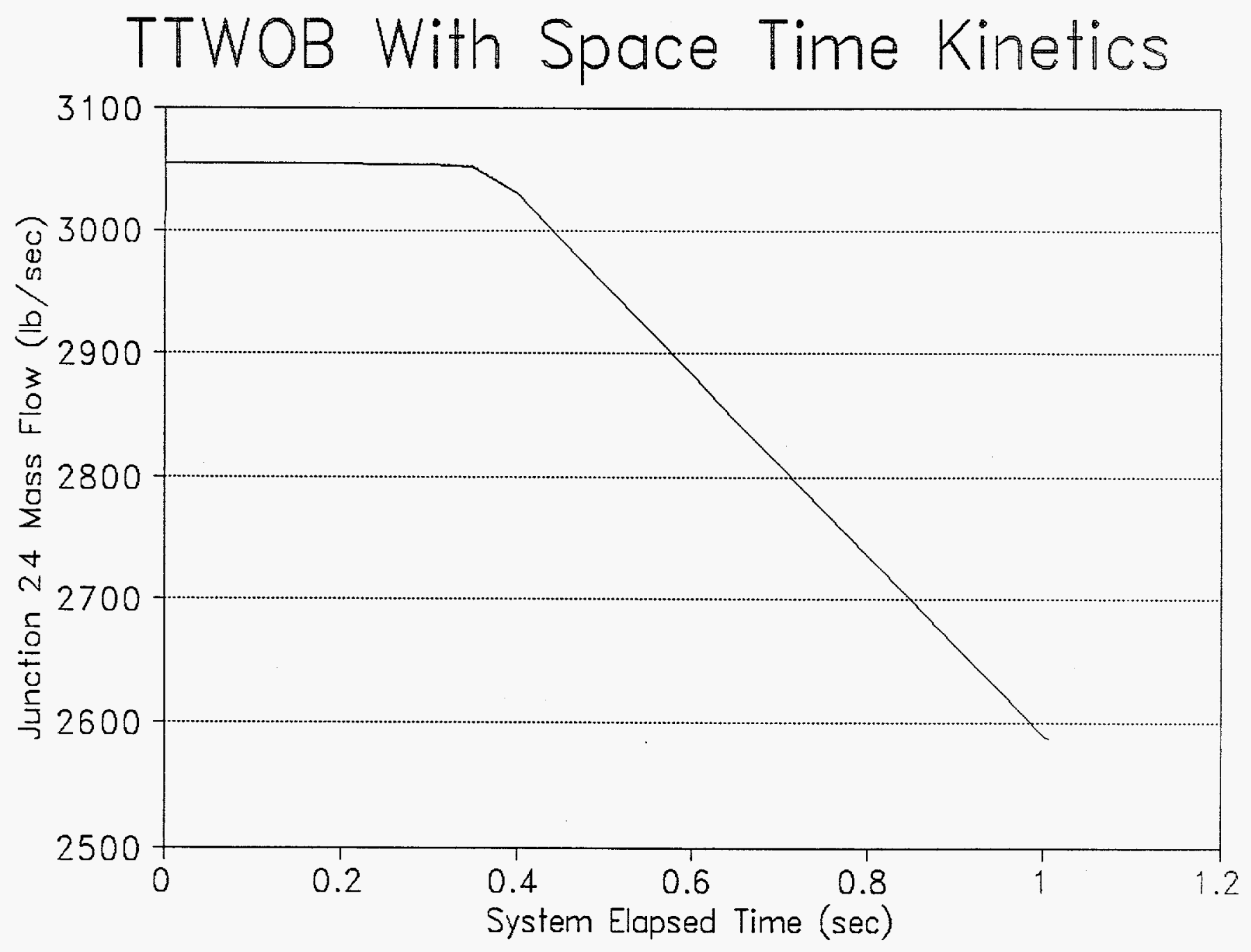


Figure 49

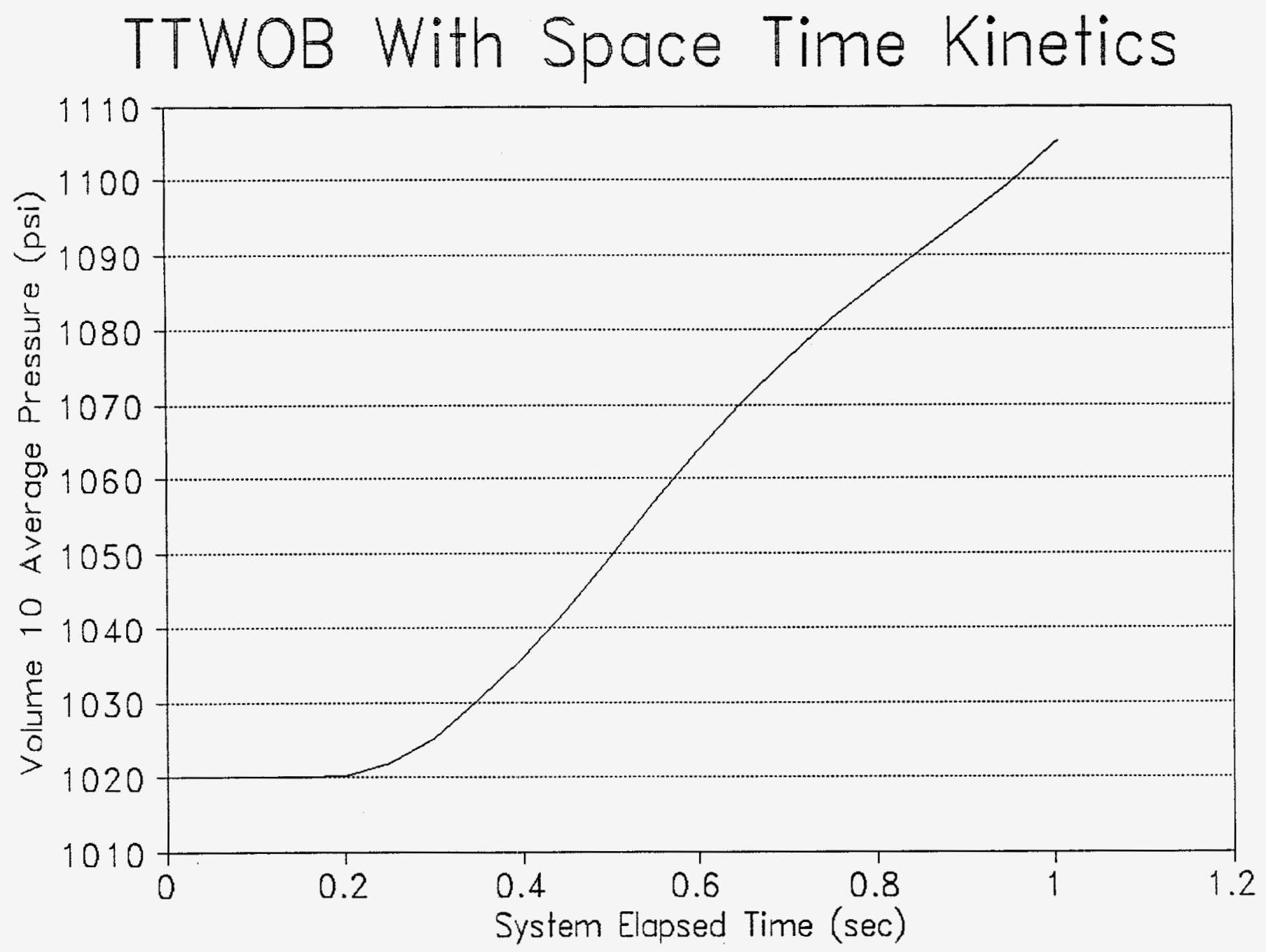


Figure 50

TTWOB With Space Time Kinetics

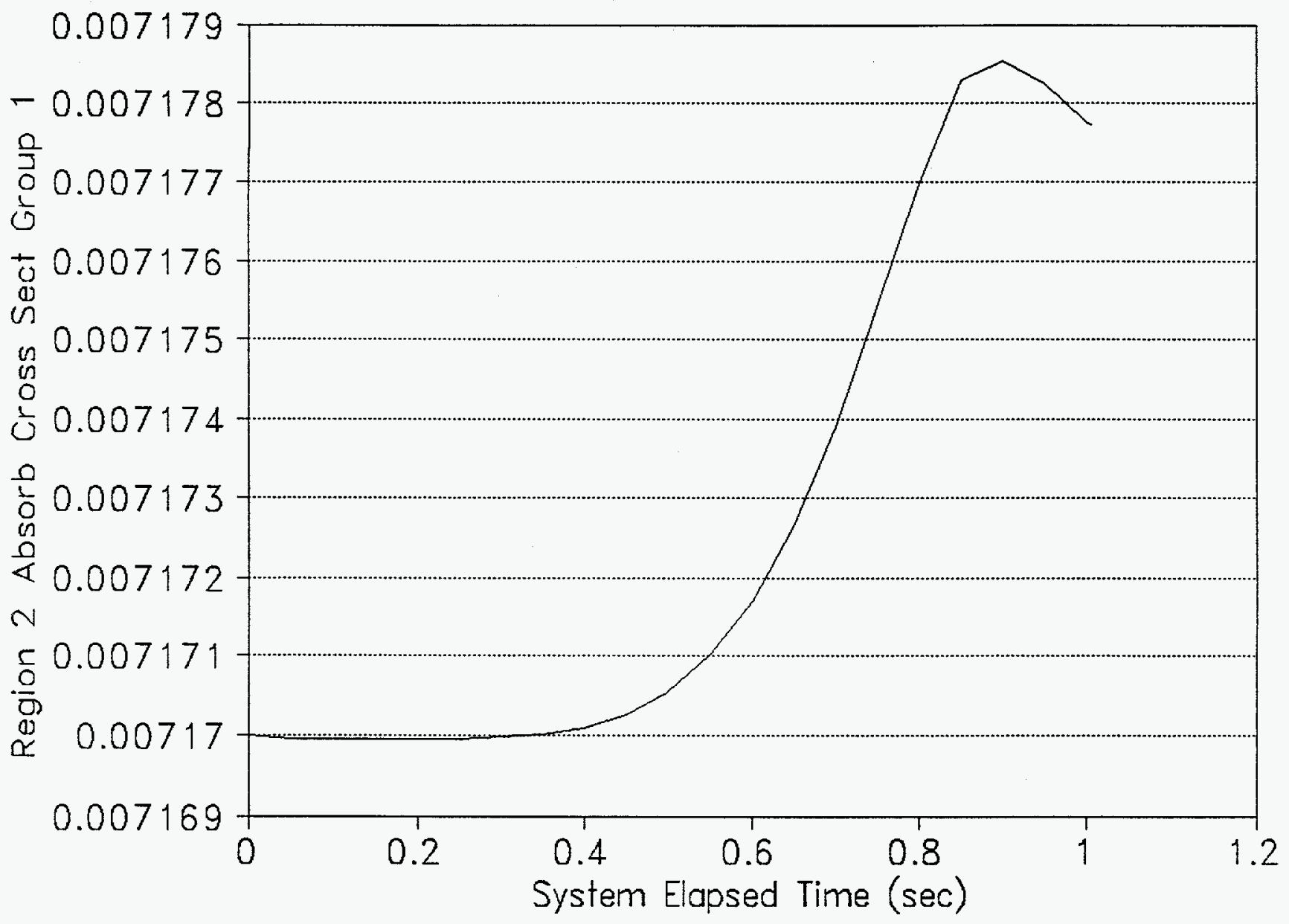


Figure 51

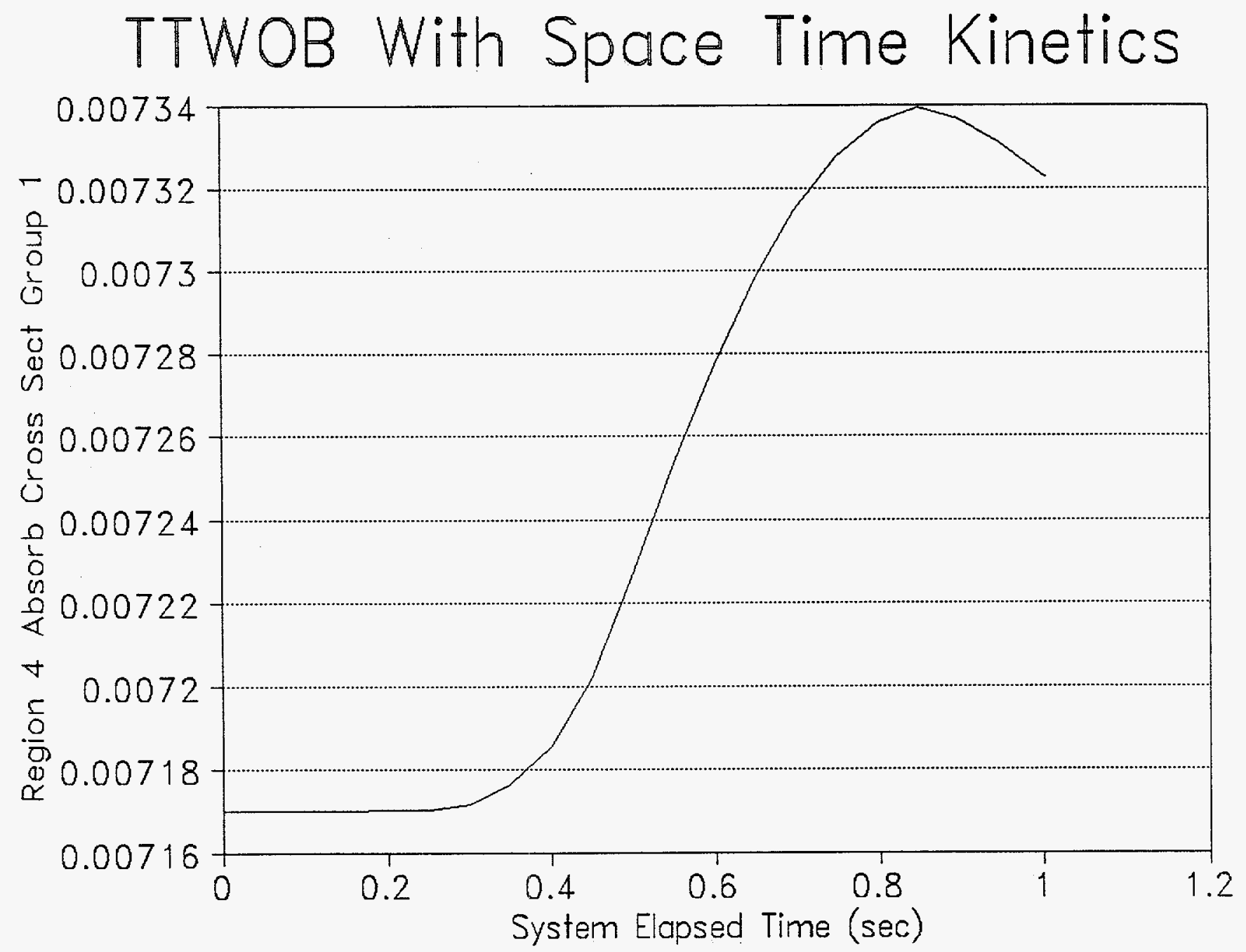


Figure 52

TTWOB With Space Time Kinetics

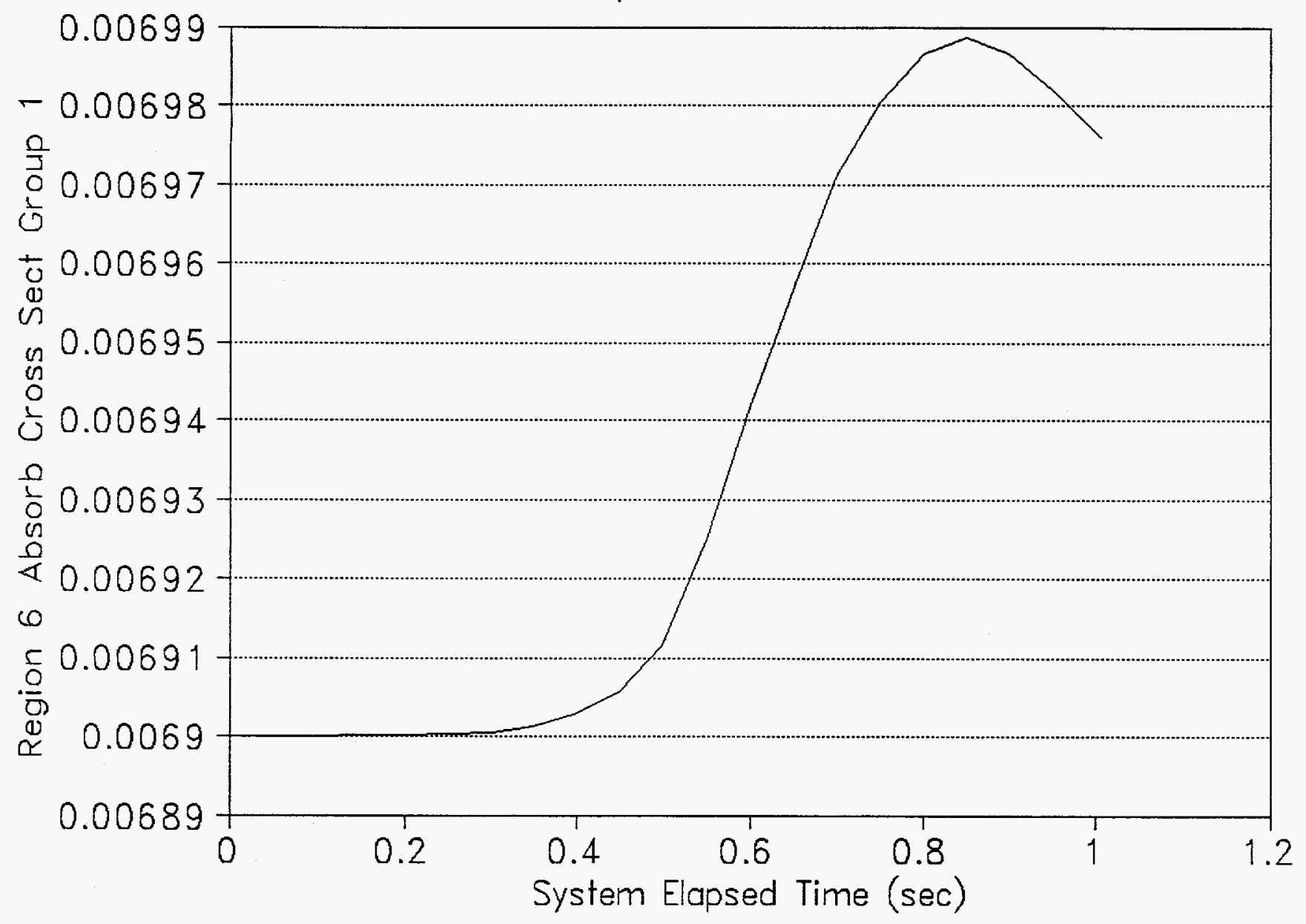


Figure 53

PWR ATWS

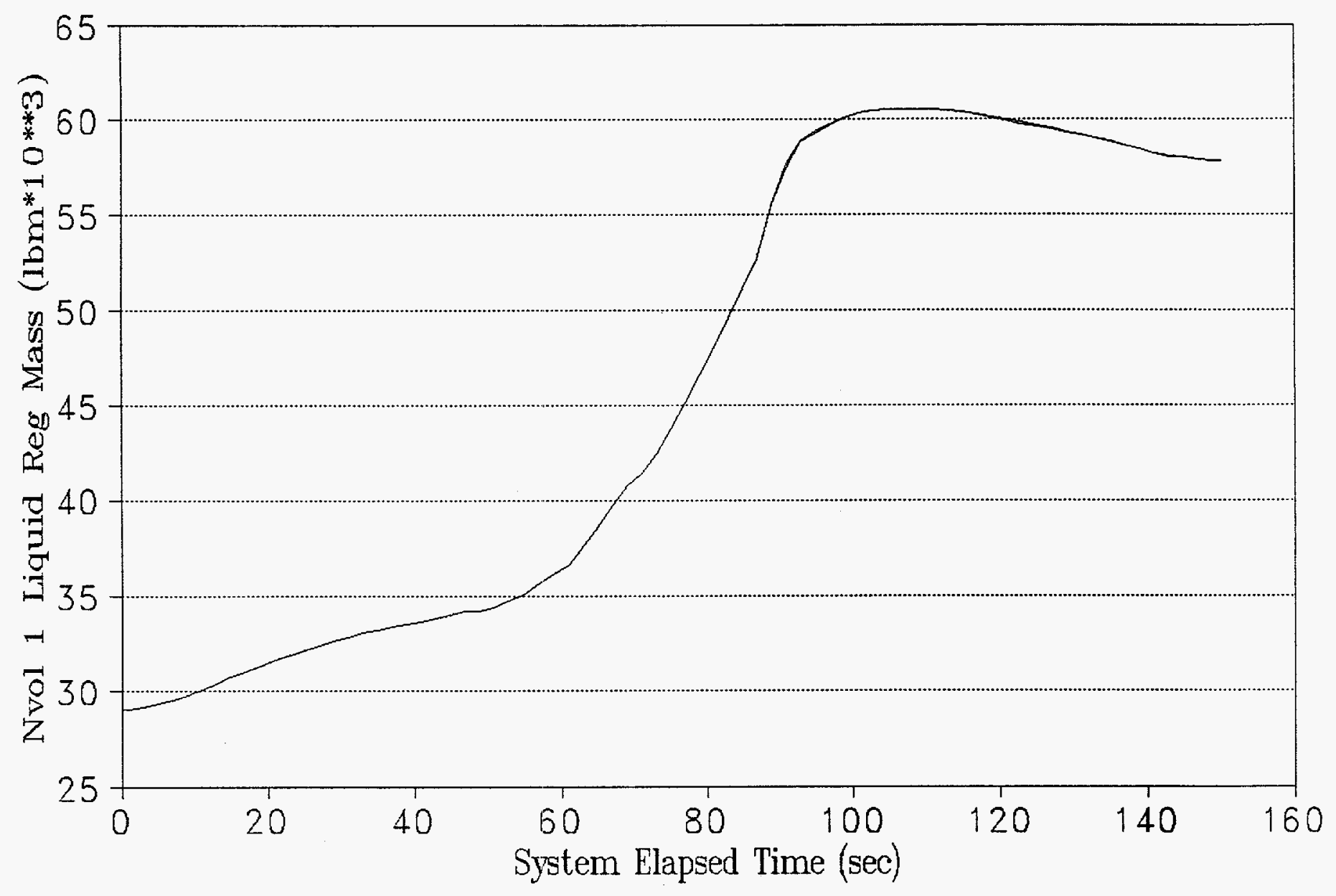


Figure 54

PWR ATWS

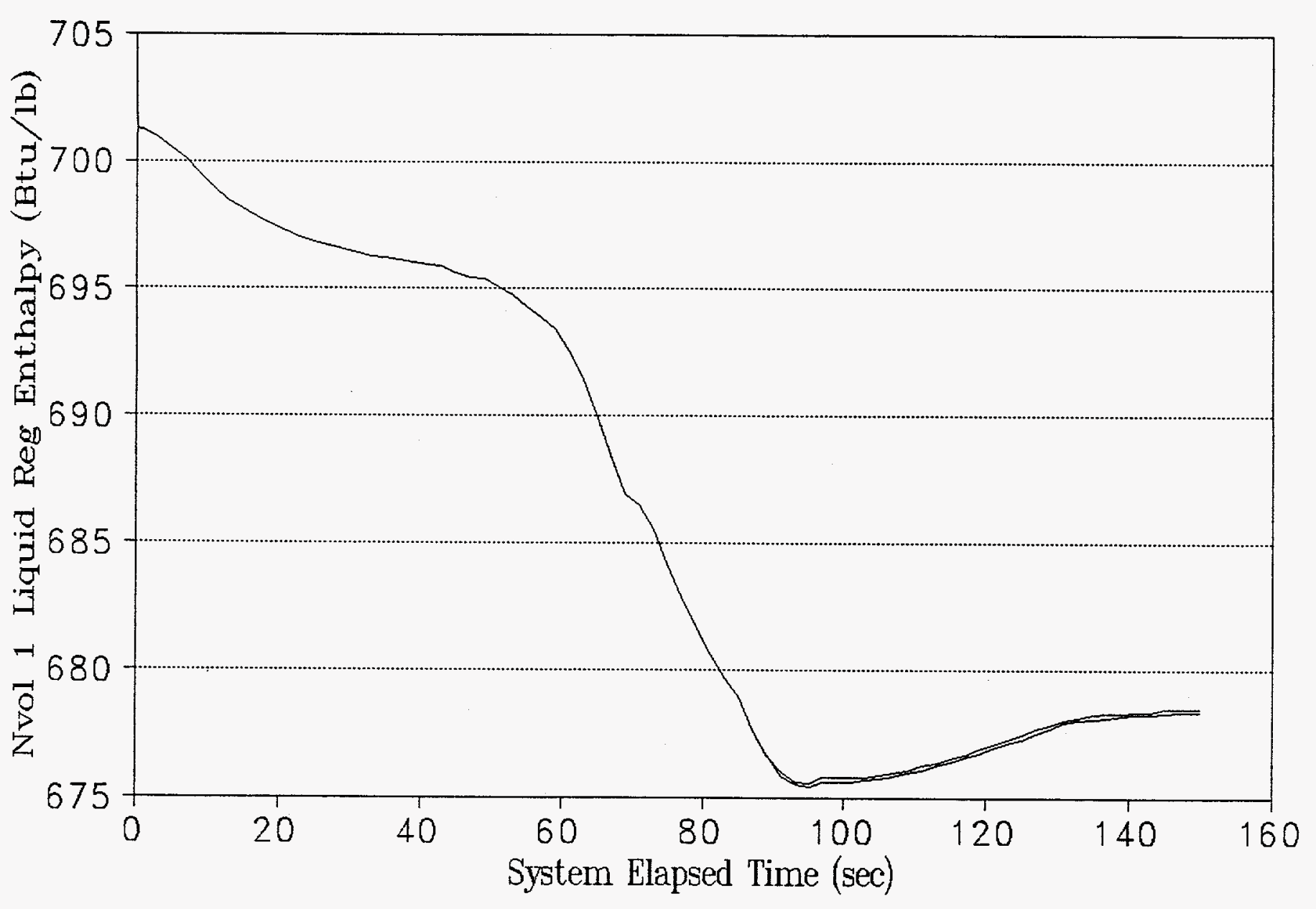


Figure 55

PWR ATWS

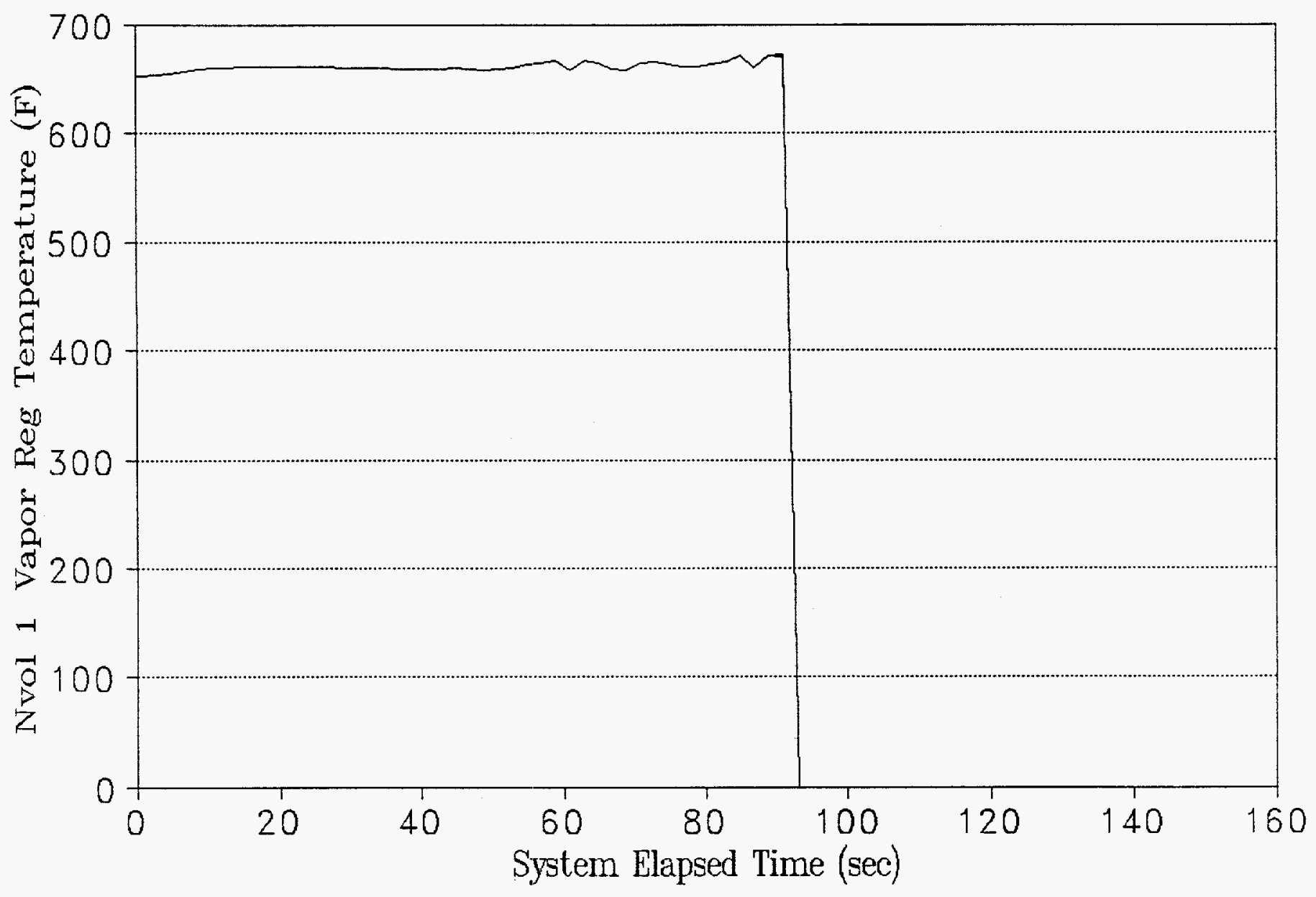


Figure 56

PWR ATWS

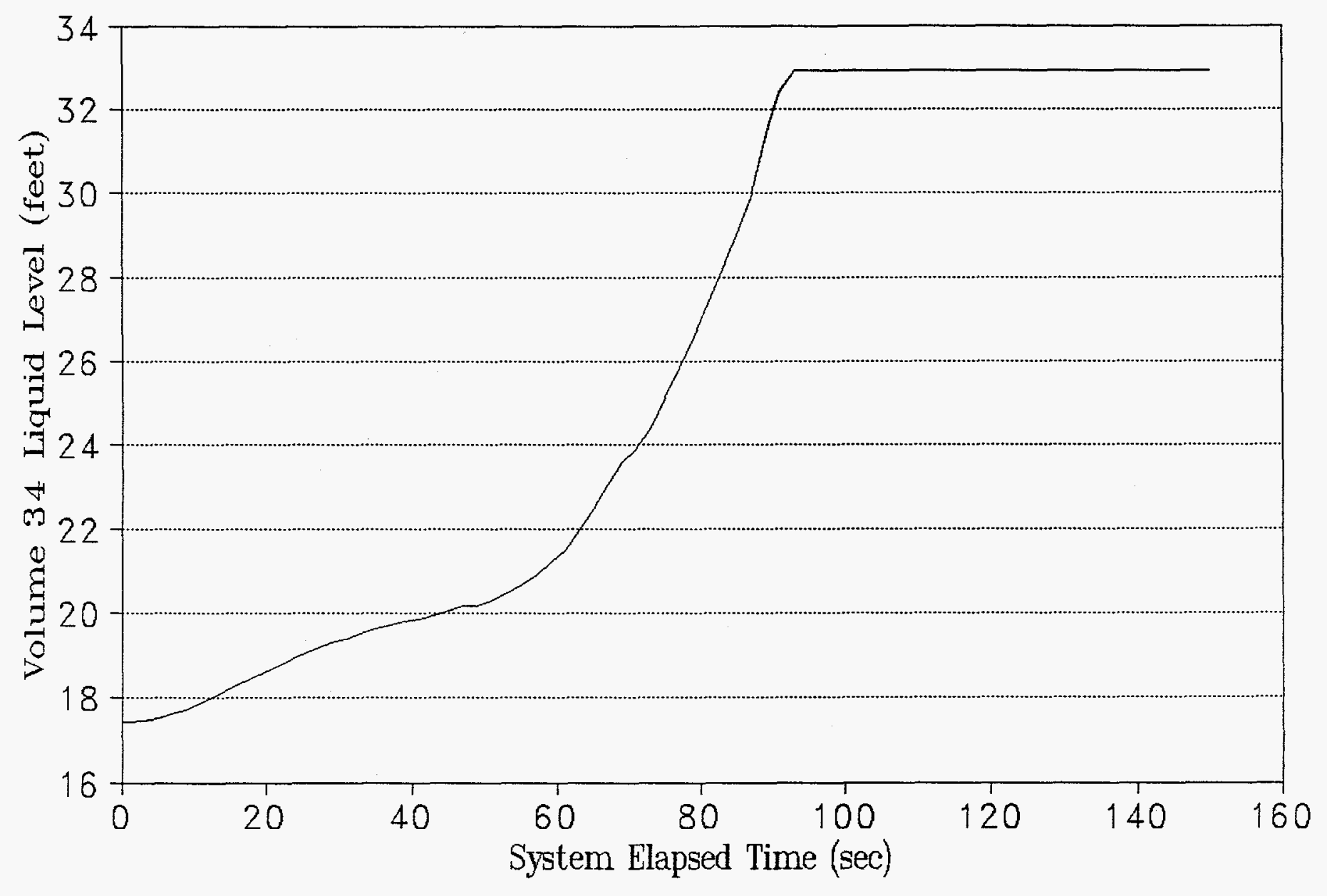


Figure 57

PWR ATWS

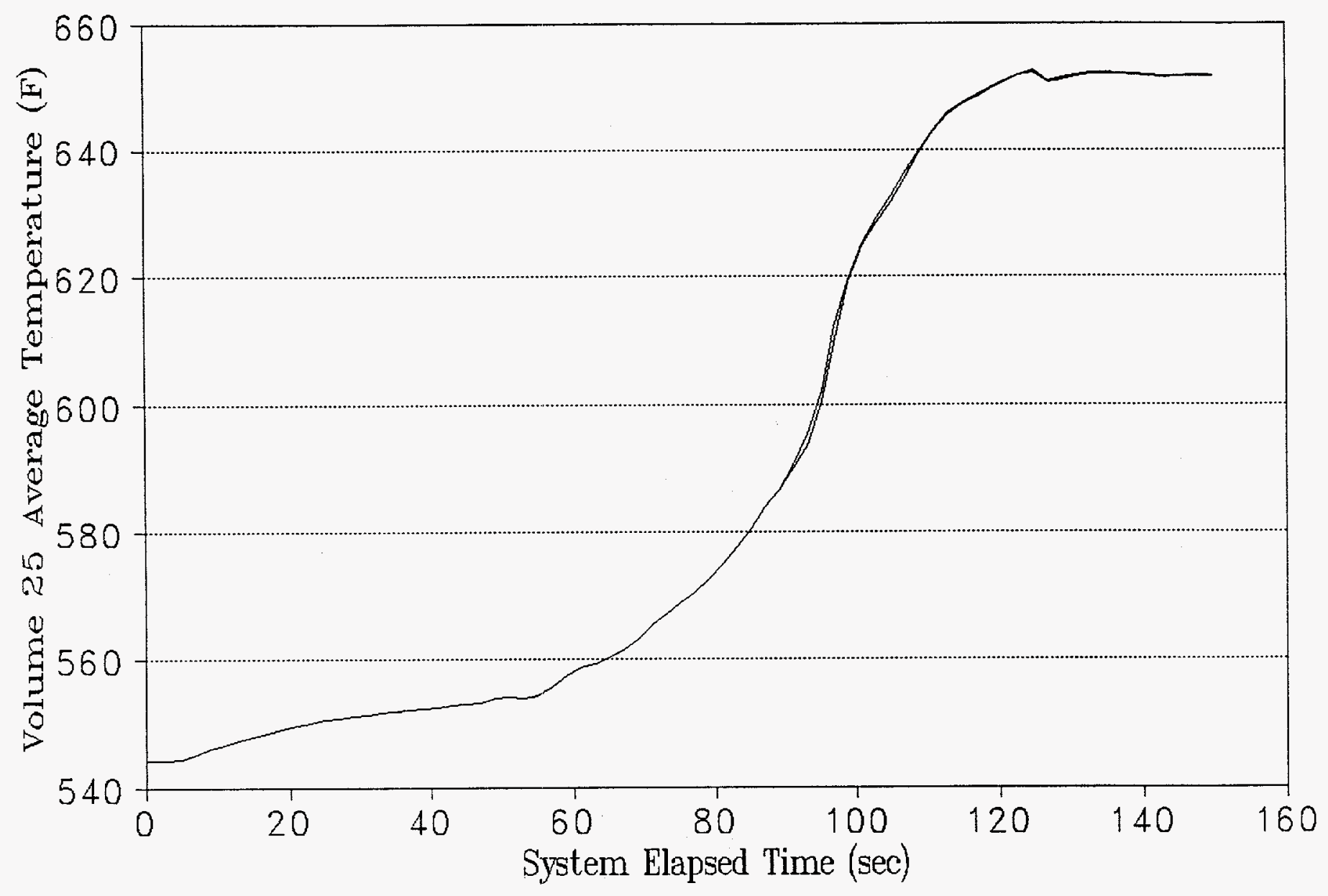


Figure 58

PWR ATWS

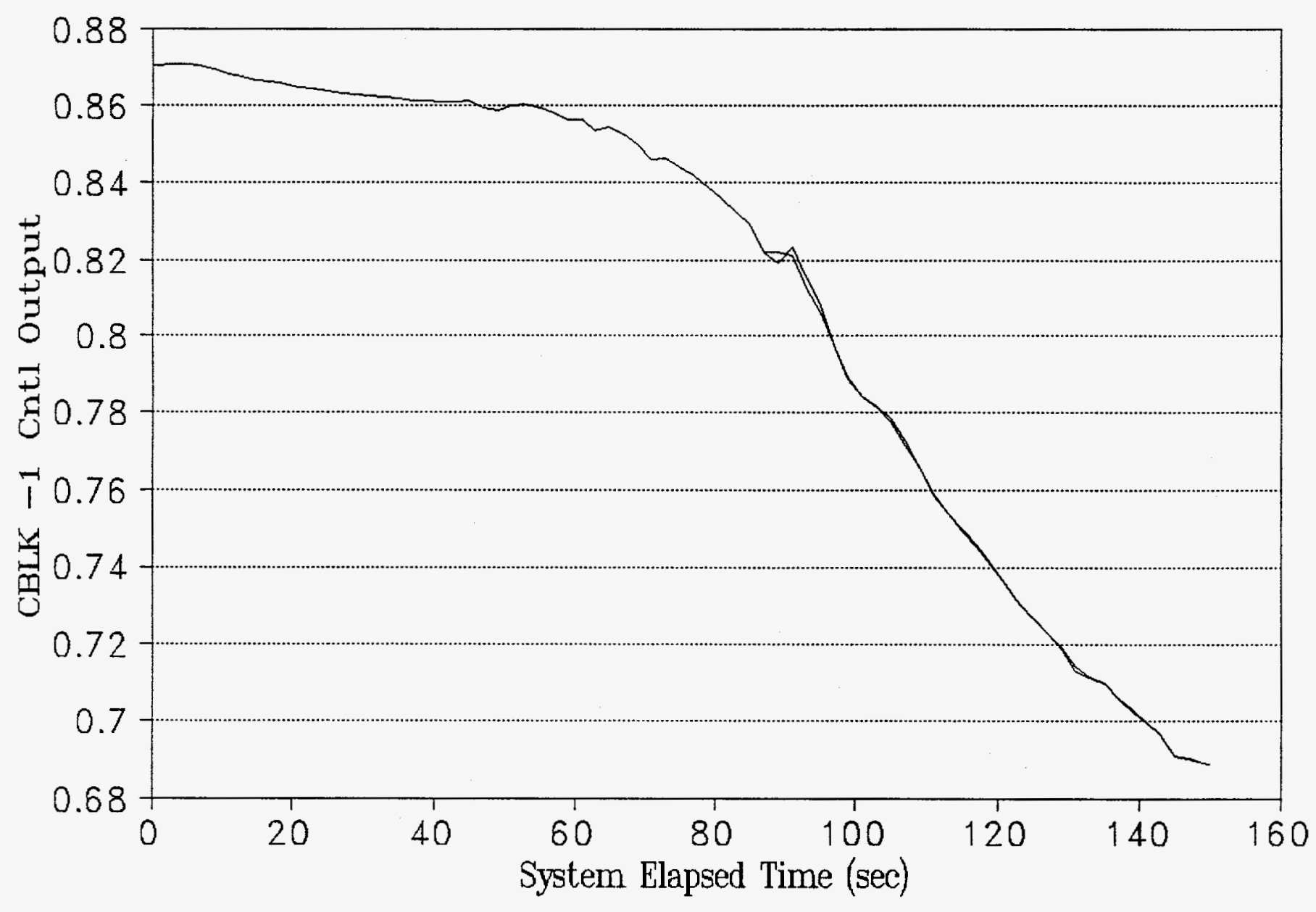


Figure 59

PWR ATWS

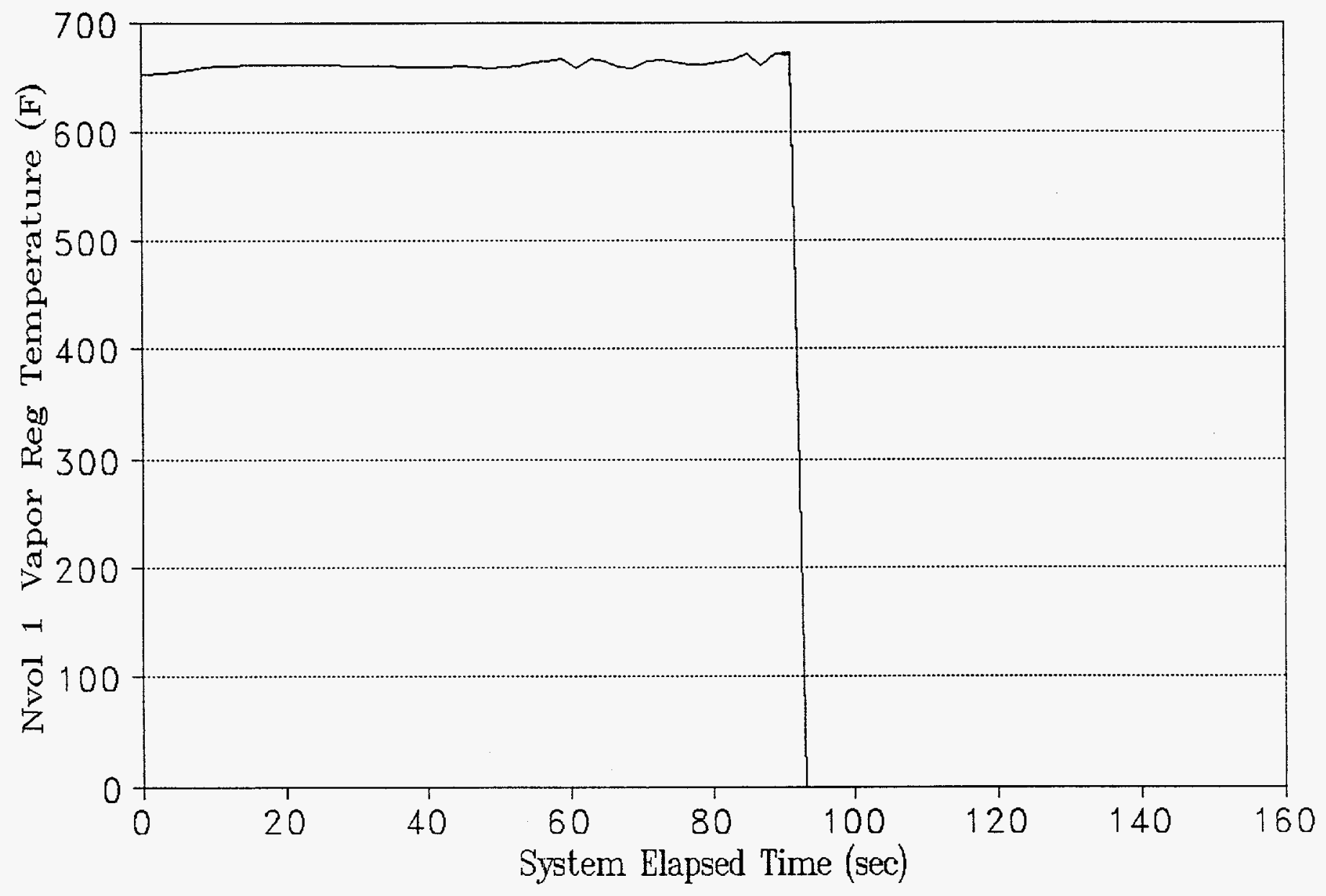


Figure 61

PWR ATWS

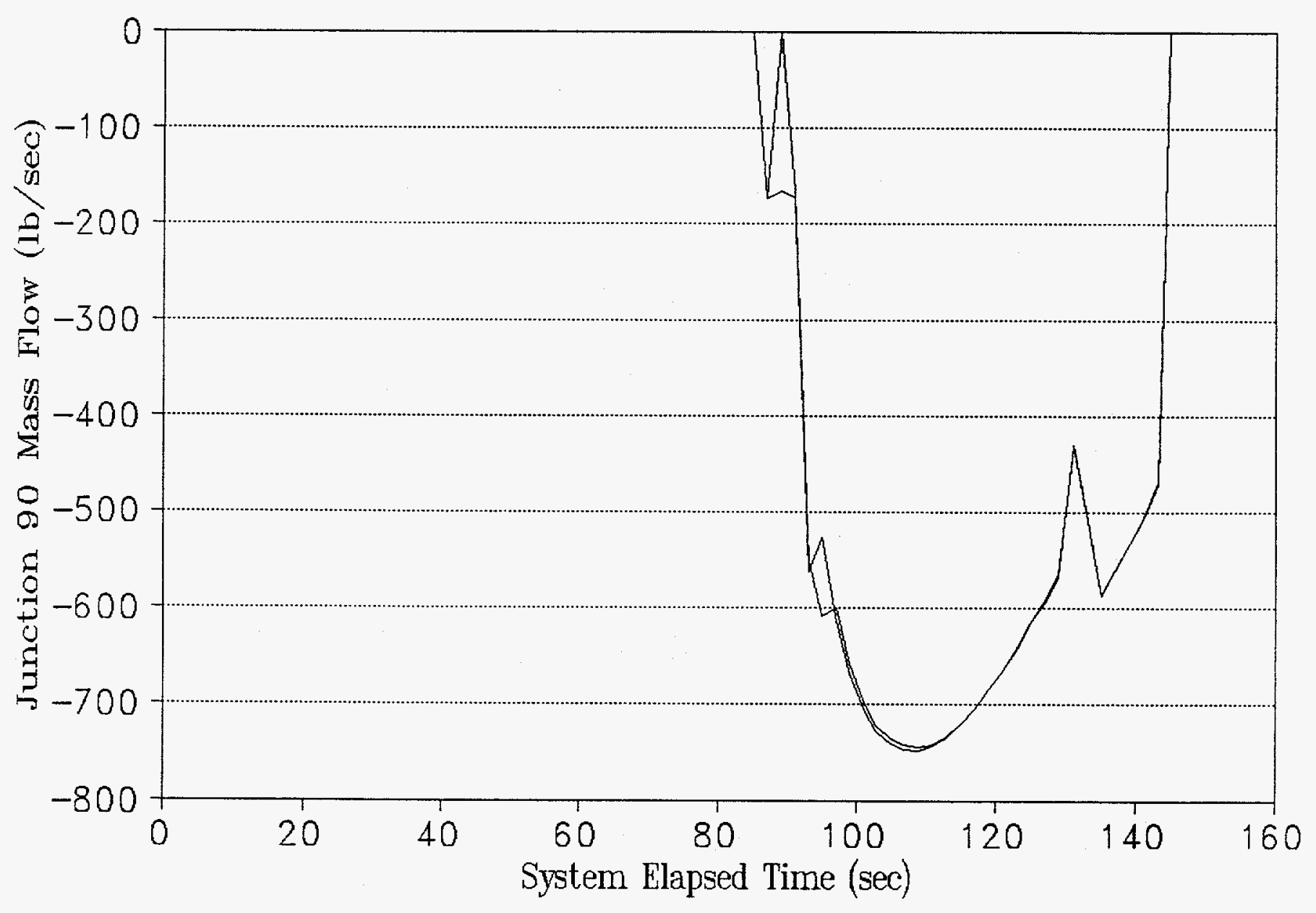


Figure 60

\section{PWR ATWS}

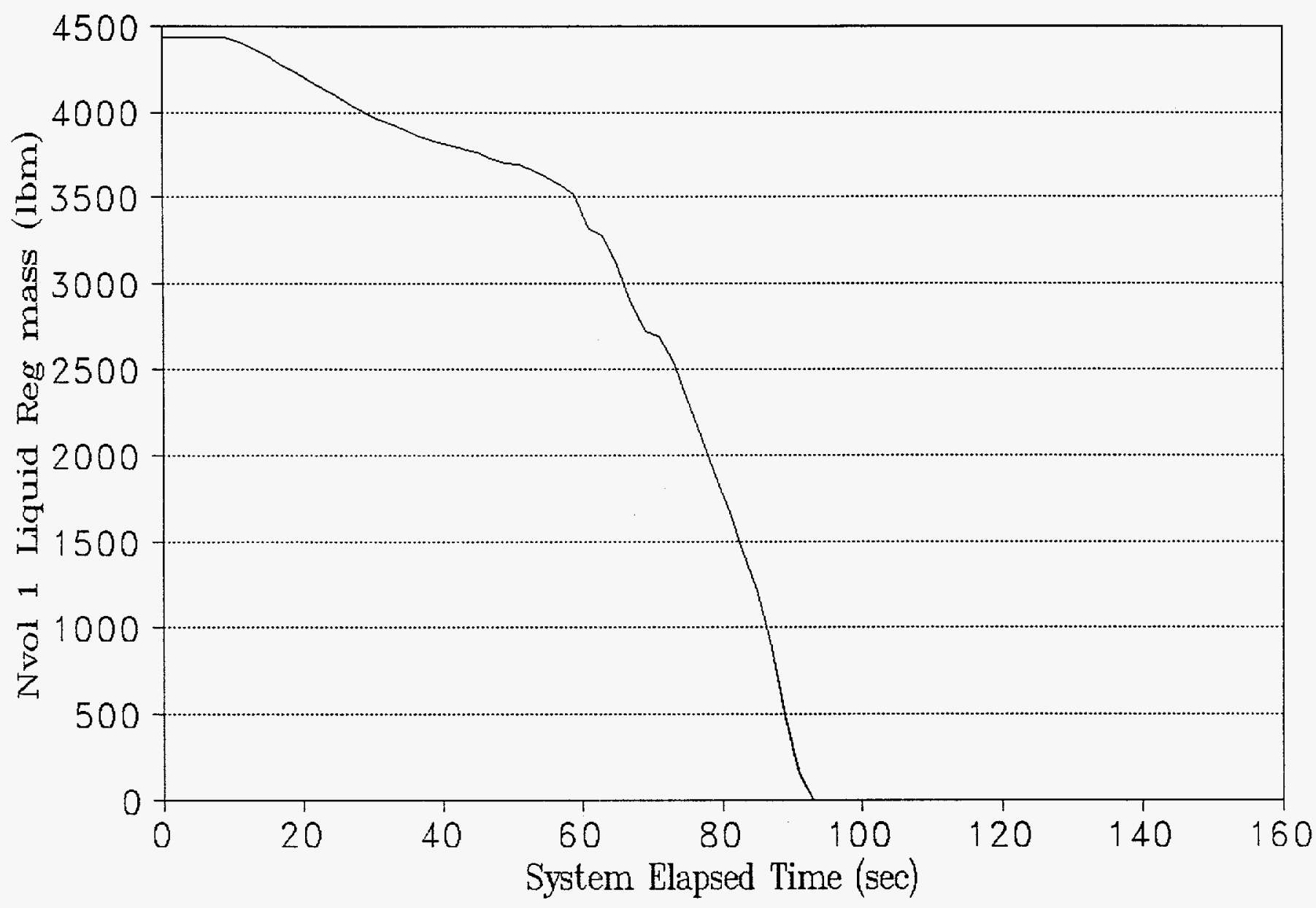


Figure 62

PWR ATWS

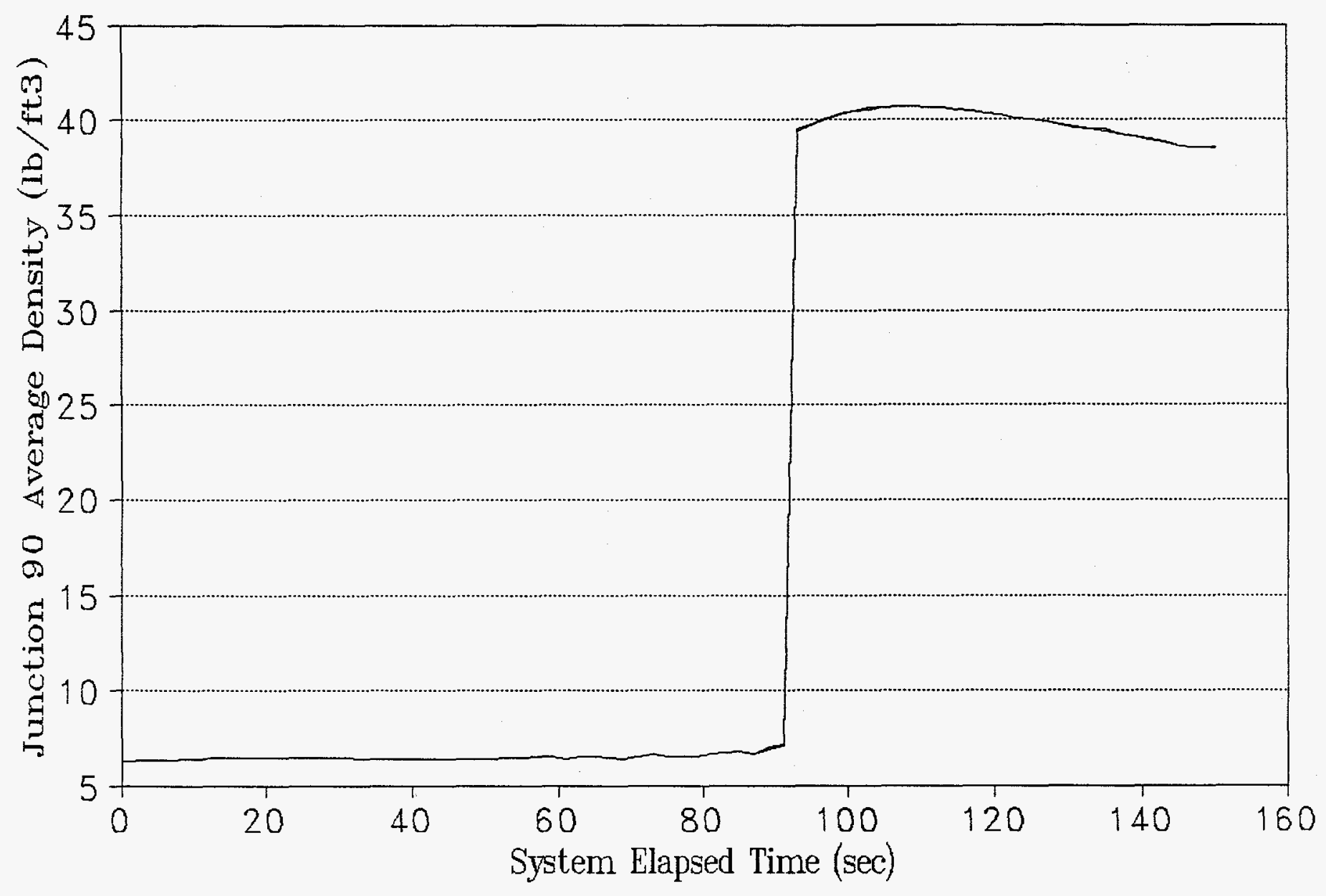


Figure 63

PWR ATWS

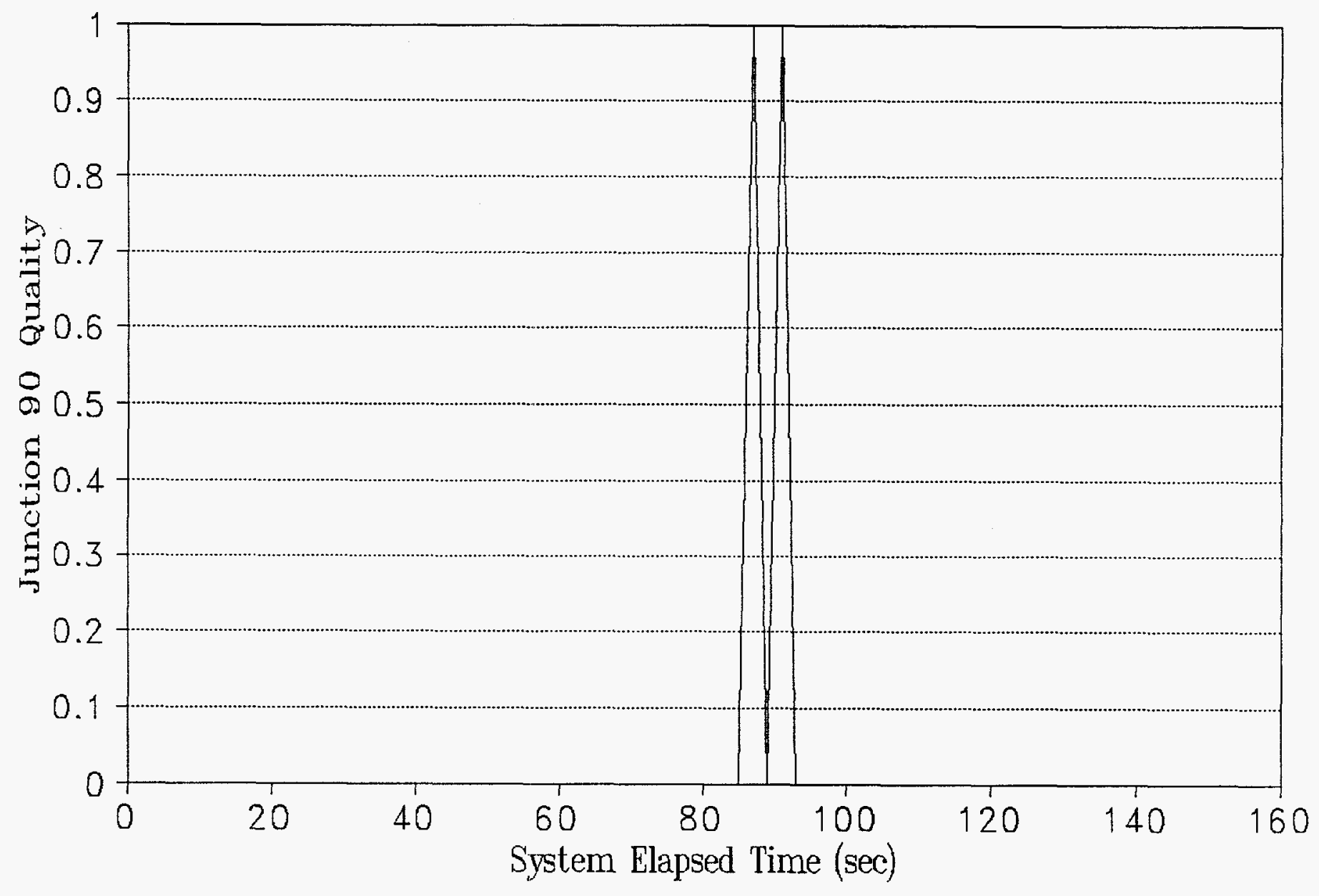


Figure 64

PWR ATWS

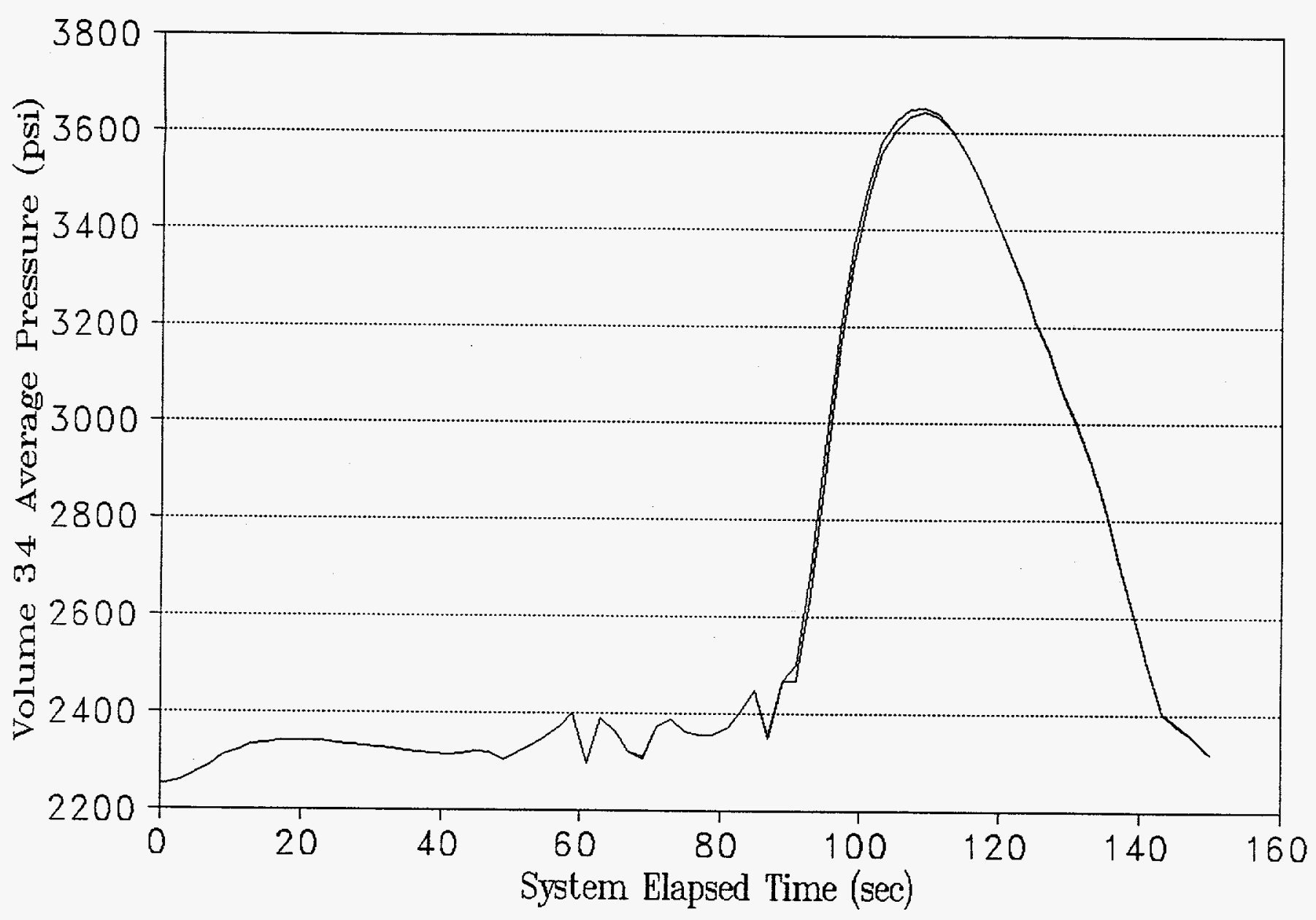


Figure 65

PWR ATWS

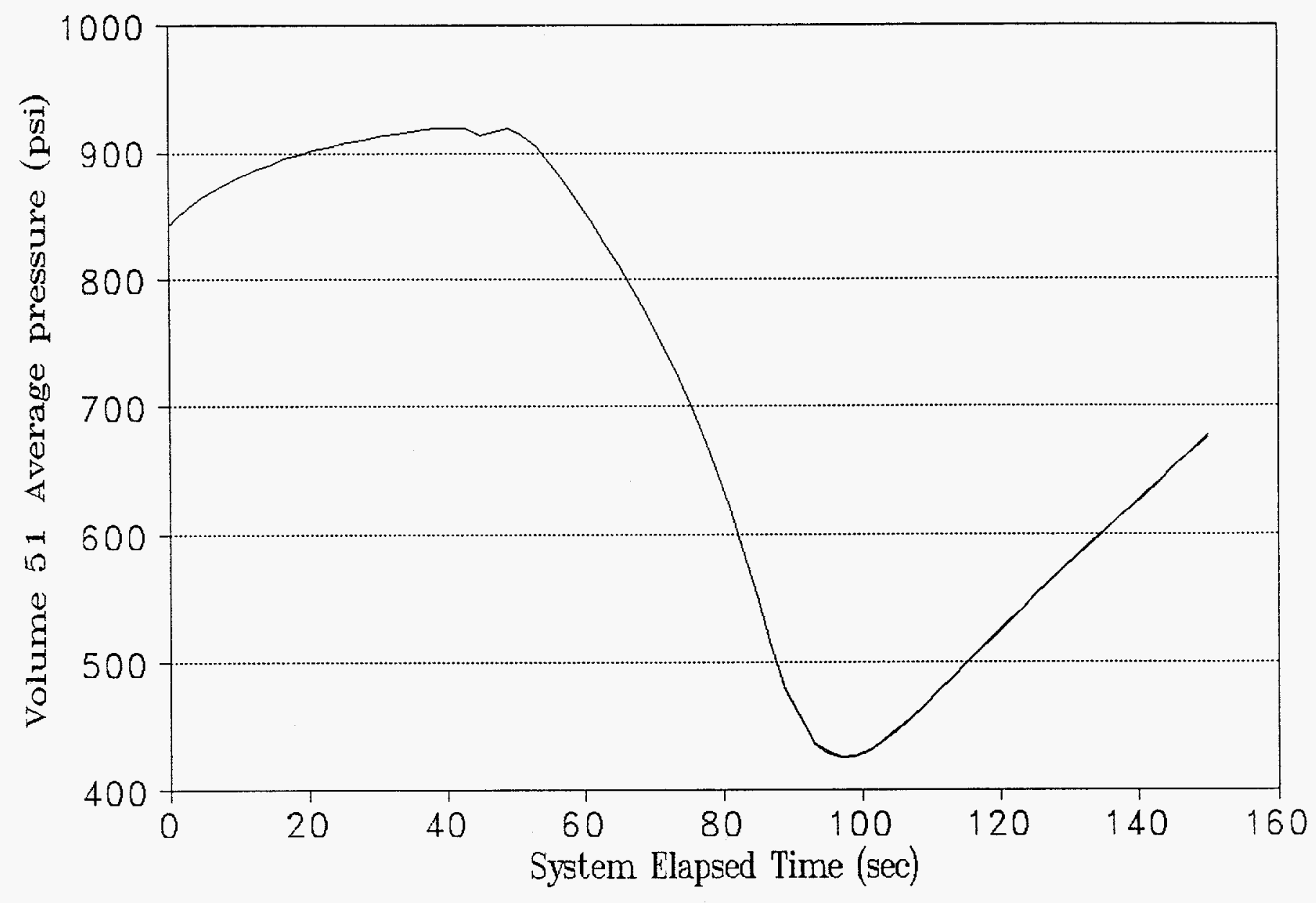


Figure 66

PWR ATWS

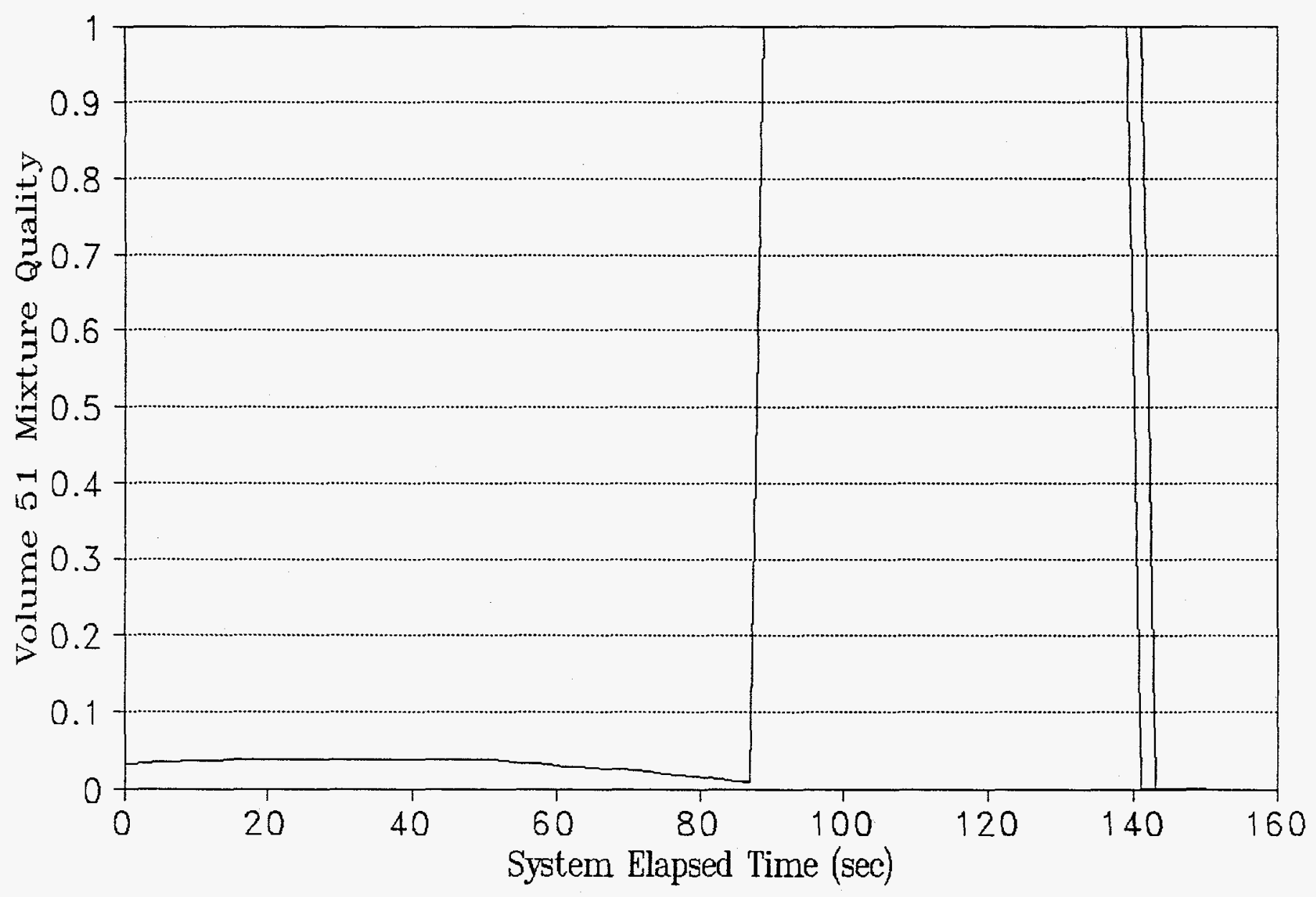




\section{APPENDIX B}

\section{ENVIRONMENTAL LIBRARY UPDATE DECK}

The environmental library for the CRAY RETRAN-02 code version was built from the CDC library source with a single update correction deck labeled with the identifier 'ident lan101'. The correction deck is listed in this Appendix. The CRAY source is written in lower case characters.

*ident lan101

*/comment purge assembly language and unnecessary decks

*/comment

*purgedk opsy $\$ c a$

*purgedk bufout

*purgedk cio

*purgedk cpar

*purgedk cvi

*purgedk dand

*purgedk dor

*purgedk ds 1

*purgedk dsr

*purgedk errm

*purgedk findep

*purgedk floatr

*purgedk frecor

*purgedk ftbio

* purgedk ftbset

*purgedk getcor

*purgedk ia

*purgedk iand

*purgedk icpsec

*purgedk inp

*purgedk ior

*purgedk is 1

*purgedk isr

*purgedk $1 \mathrm{cmbuf}$

*purgedk $1 \mathrm{cmdum}$

*purgedk link

* purgedk maskf

*purgedk move

*purgedk movei

*purgedk notim

*purgedk pack

*purgedk pckupk

* purgedk reduce

*purgedk symbol

*purgedk tims 


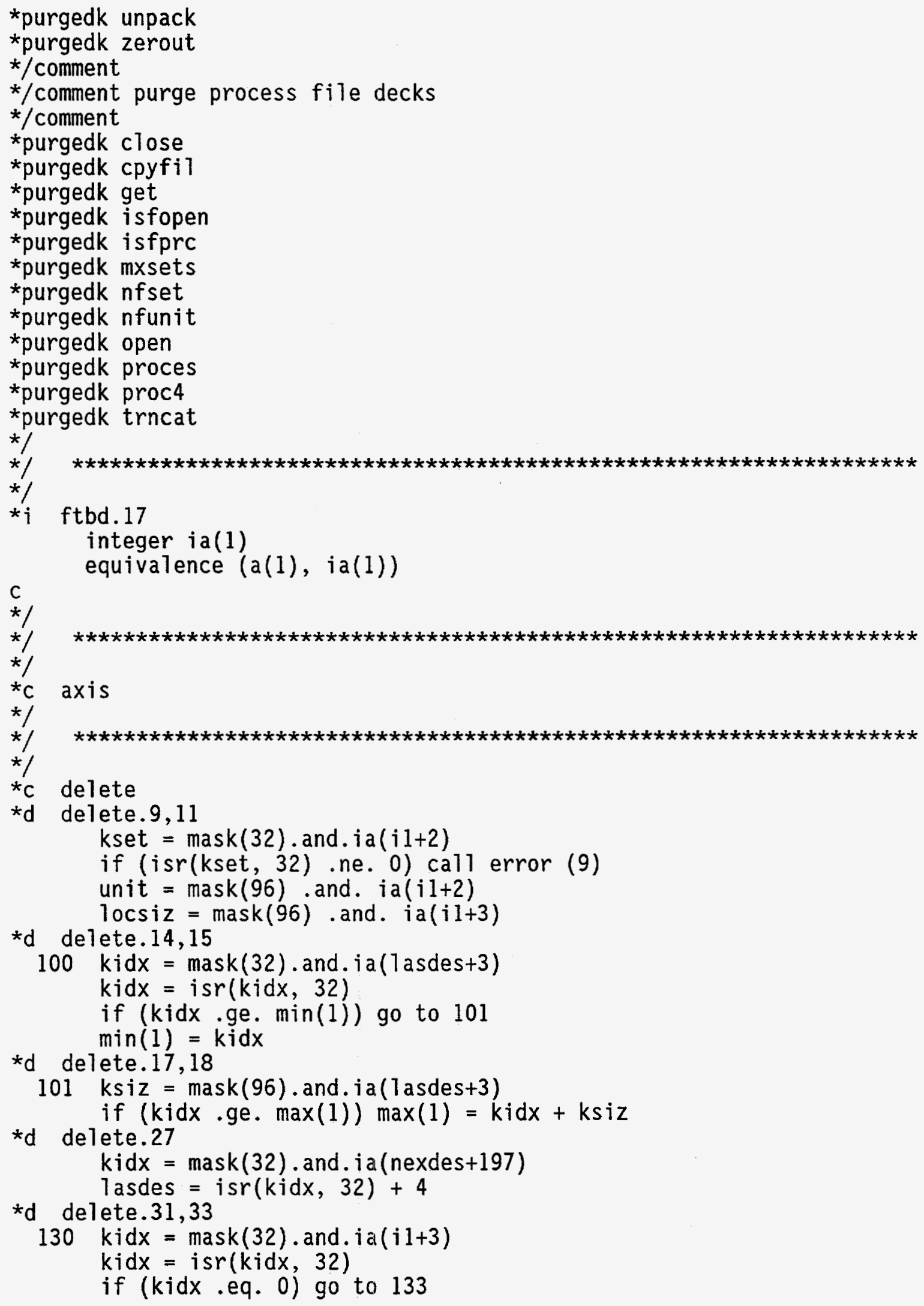


*d delete. 35

if (kidx .ge. min(unit)) go to 131

$\min ($ unit) $=k i d x$

131 if (kidx .ge. $\max ($ unit)) $\max ($ unit) $=k i d x+1$ ocsiz

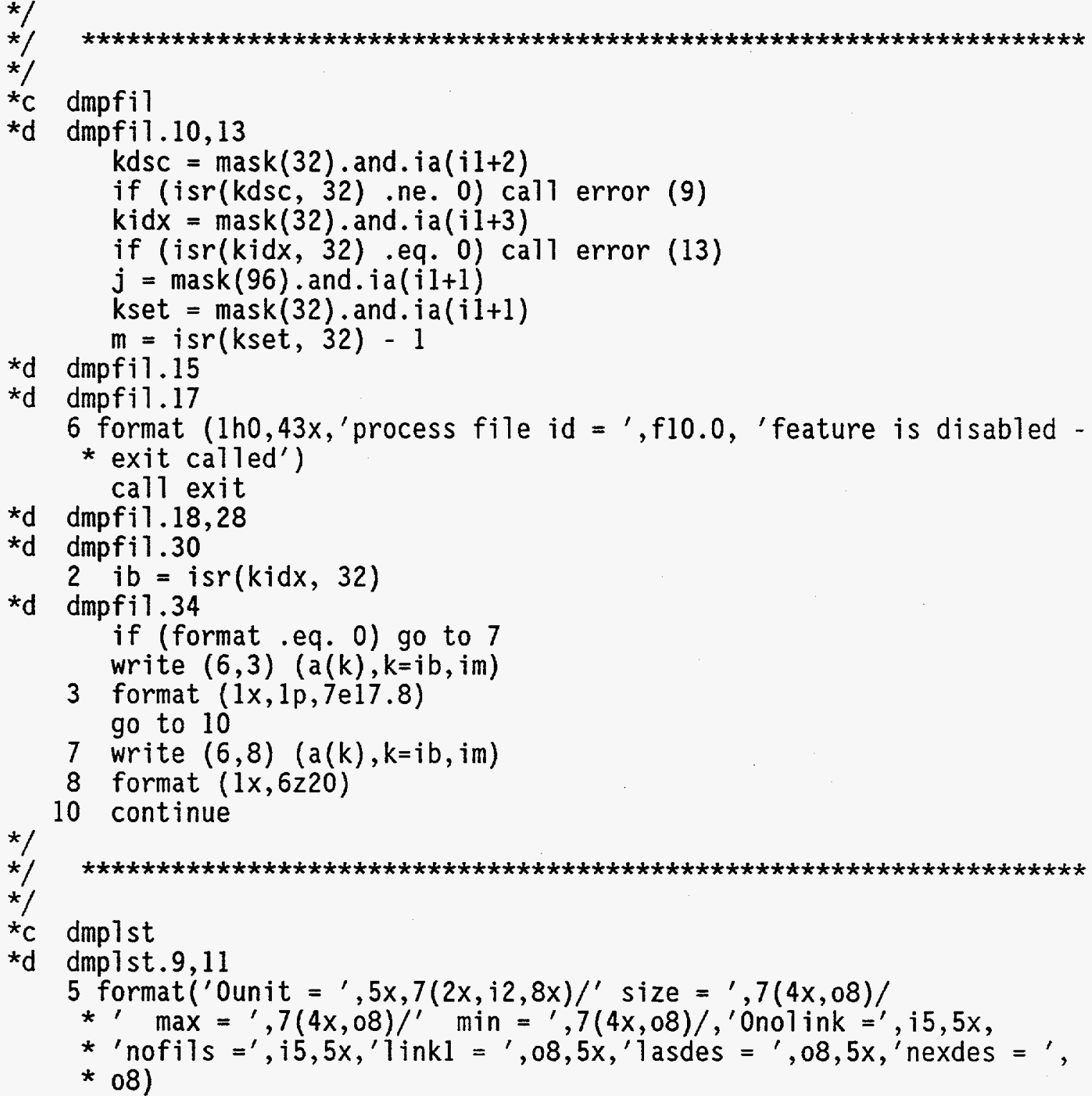

$* d$ dmplst.22,27

$m l=i \operatorname{sr}(\operatorname{mask}(32)$. and $. i a(j+1), 32)$

$m 2=\operatorname{mask}(96) \cdot$ and $\cdot i a(j+1)$

$m 3=i \operatorname{sr}(\operatorname{mask}(32) \cdot$ and. $i a(j+2), 32)$

$m 4=\operatorname{mask}(96) \cdot$ and $\cdot i a(j+2)$

$m 5=i \operatorname{sr}(\operatorname{mask}(32)$. and $. i a(j+3), 32)$

$m 6=\operatorname{mask}(96)$. and.$i a(j+3)$

*d dmp Tst.33,37

6 format $\left(/ / 65 x,{ }^{\prime} 1\right.$ ink' $^{\prime}, i 3 / 65 x,^{\prime * * * * * * * \prime} / / 2\left(8 x,^{\prime}\right.$ id setsiz nosets

* idsdsc unit filidx filsiz', 4x))

8 format ( $1 x, f 9.0,3 x, i 6,2 x, i 6,2 x, 08,3 x, i 2,3 x, 08,2 x, 08)$ 
9 format (' + ' $, 67 x, f 9.0,3 x, i 6,2 x, i 6,2 x, 08,3 x, i 2,3 x, 08,2 x, 08)$

*1

$1 \quad n 1=i \operatorname{sr}(\operatorname{mask}(32)$. and.ia(n2), 32)

*/

$* 1$

${ }^{*} \mathrm{c}$ dscrib

*d dscrib.16,17

$$
\mathrm{a}(\text { nexdes }+1)=0.0 \mathrm{e} 0
$$

$i a($ nexdes +1$)=$ shiftl $($ setsiz, 32) .or. nosets

*d dscrib. 19

$$
\text { ia }(\text { nexdes }+2)=\text { unit }
$$

*d dscrib.27

$$
i a(\text { nexdes }+1)=\operatorname{mask}(32) \cdot \text { and } \cdot i a(\text { nexdes }+1)
$$

*d dscrib. 29,31

$$
\begin{aligned}
i a(\text { nexdes }+2) & =1 \\
a(\text { nexdes }+3) & =0.0 \\
i a(\text { nexdest3) } & =\operatorname{shift}(i 1,32) \text {.or. } 200
\end{aligned}
$$

*d dscrib. 36

$$
\begin{aligned}
& i a(\text { nexdes }+197)= \\
& (\operatorname{mask}(96) . \text { and.ia }(\text { nexdes }+197)) \\
& \text {.or. shiftl }(1 \text { asdes }-4,32)
\end{aligned}
$$

$*$

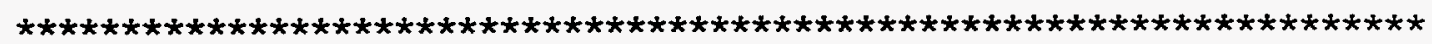

$* /$

${ }^{*} \mathrm{C}$ envid

*d envid.11

data usr / 8hlan13/90/

*1

*/

${ }^{*} c$ error

*d error. 9

call fabend

*/

$*$

$* 1$

*c fabend

$* j$ fabend. 3

integer unit

*d fabend.6,9

data unit /6htape6/

*d fabend.11,30

call trbk (unit)

${ }^{*} \mathrm{c}$ fort

${ }^{*} \mathrm{~d}$ fort.6,60

$* 1$

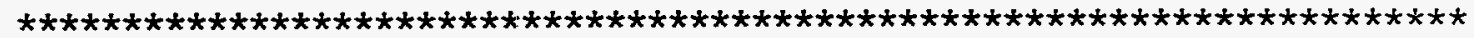

$* /$

${ }^{*} \mathrm{C}$ idfind

${ }^{*} \mathrm{~d}$ idfind. 20 


$$
i=i \operatorname{sr}(\operatorname{mask}(32) \cdot \text { and. } i a(i+197), 32)
$$

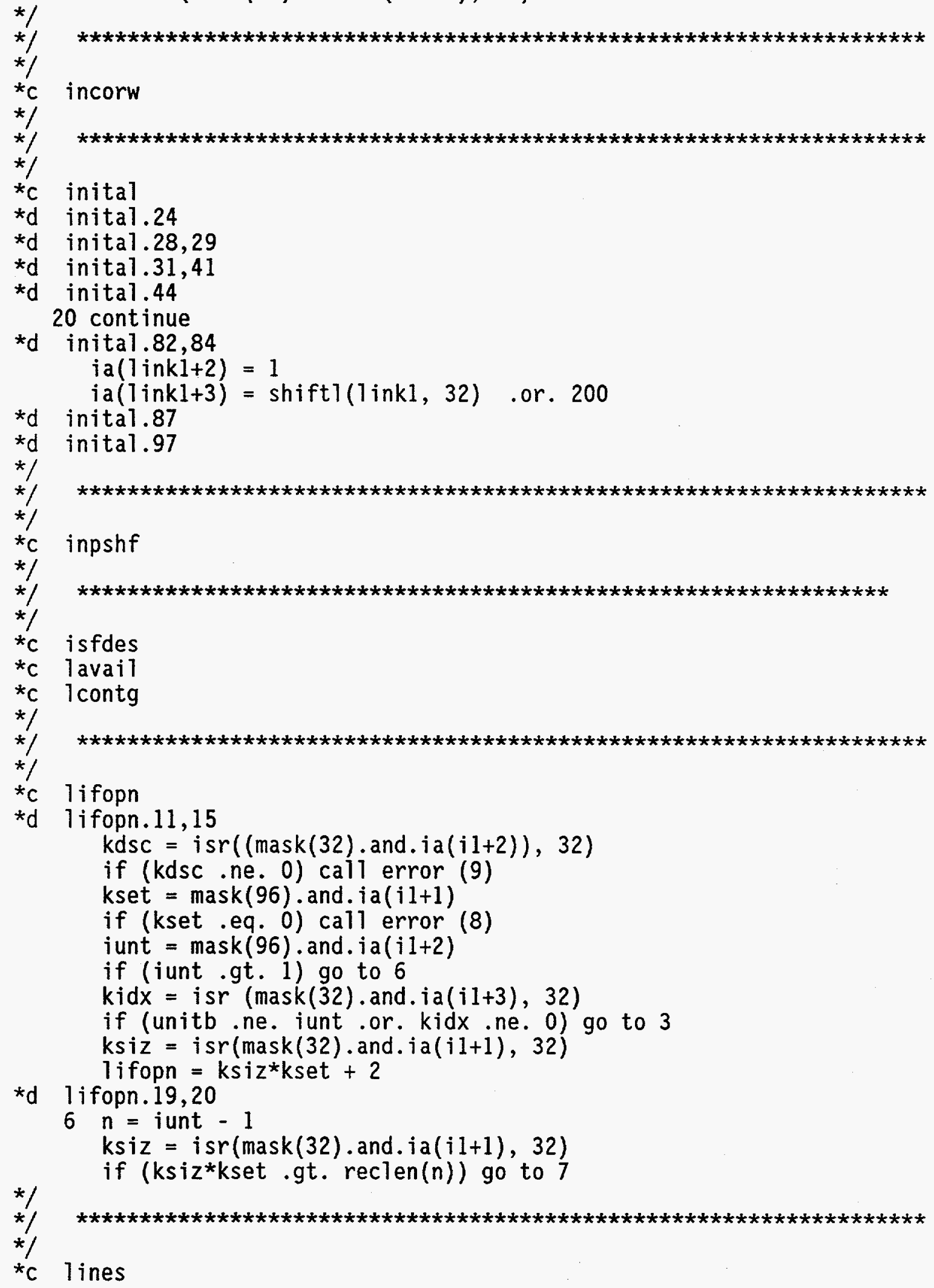




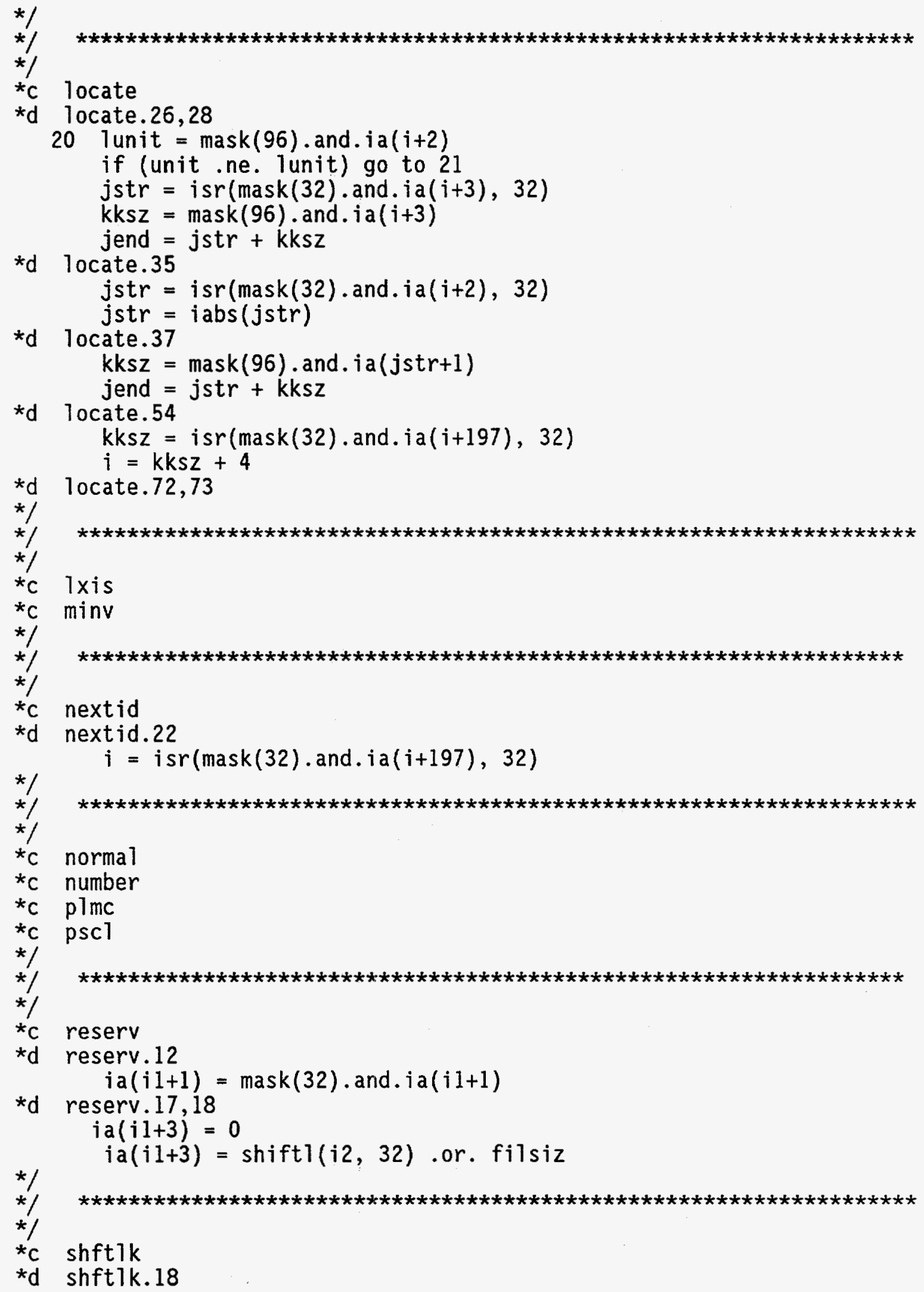


$j=i s r(\operatorname{mask}(32)$.and.ia $(1+3), 32)$

*d shftlk.29

$$
i a(1+2)=\operatorname{mask}(32) \text {.and } i a(1+2)
$$

*d shftlk.32

$$
i a(1+2)=(\operatorname{mask}(32) \cdot \text { and } . i a(1+2)) \text {.or. } 1
$$

$i a(1+3)=(\operatorname{mask}(96) \cdot$ and.ia( $(1+3))$.or. $\operatorname{shift} 1(i 2,32)$

*d shftlk. 48,49

kidx $=i \operatorname{sr}(\operatorname{mask}(32)$. and.ia(i2+199), 32)

$k=k i d x+196$
$i a(k+1)=(\operatorname{mask}(96) \cdot$ and.ia(k+1)) .or. $\operatorname{shift}(i 2+192,32)$

$\begin{array}{ll}* / 1 & \\ * & \\ * & \\ * & \end{array}$

$*_{d}$ shift.13

kset $=\operatorname{mask}(96)$. and.$i a(i 1+1)$

if (kset .ne. 0) call error (43)

*d shift.15

$$
1 \quad i a(i 1+2)=\operatorname{mask}(32) \text {.and. } i a(i 1+2)
$$

$*_{d}$ shift. 20,21

kidx $=i \operatorname{sr}(\operatorname{mask}(32)$.and.ia(i1+3), 32)

$k s i z=\operatorname{mask}(96)$. and.$i a(i 1+3)$

if $(k i d x .7 t \cdot \min (1)) \min (1)=k i d x$

$\star_{d}$ shift. 25

if (kidx.ge. $\max (1)) \max (1)=k i d x+k s i z$

*d shift. 27

if (i2 .eq. kidx) go to 140

$1=\operatorname{mask}(96)$. and. $i a(i 1+3)$

$\mathrm{ksiz}=1$

*d shift.29

$$
j=k i d x
$$

*d shift. 43,48

$130 i a(i 1+3)=\operatorname{mask}(96) \cdot$ and.$i a(i 1+3)$.or. $\operatorname{shiftl}(i 2,32)$

140 size $(1)=\operatorname{size}(1)-f l s z+k s i z$

$i a(i 1+3)=\operatorname{mask}(32)$. and.$i a(i 1+3)$.or. flsz

$i a(i 1+1)=\operatorname{mask}(96)$. and.$i a(i 1+1)$.or. $\operatorname{shift1}(f 1 s z, 32)$

$i a(i 1+2)=\operatorname{mask}(32)$. and.$i a(i 1+2)$. or .1

indx $=i \operatorname{sr}(\operatorname{mask}(32) \cdot$ and.ia(i1+3), 32)

$*_{\mathrm{d}}$ shift. 51,53

$150 \mathrm{kidx}=i \operatorname{sr}(\operatorname{mask}(32)$. and. $i a(i 1+3), 32)$

$k s i z=\operatorname{mask}(96)$. and.$i a(i 1+3)$

if (f1sz,gt. ksiz) go to 160

if $(\max (1) .1 e . k i d x) \max (1)=k i d x+k s i z$

$*_{d}$ shift. 55

if $(\min (1) \cdot g e . k i d x) \min (1)=k i d x+f 1 s z$

$160 \min (1)=k i d x$

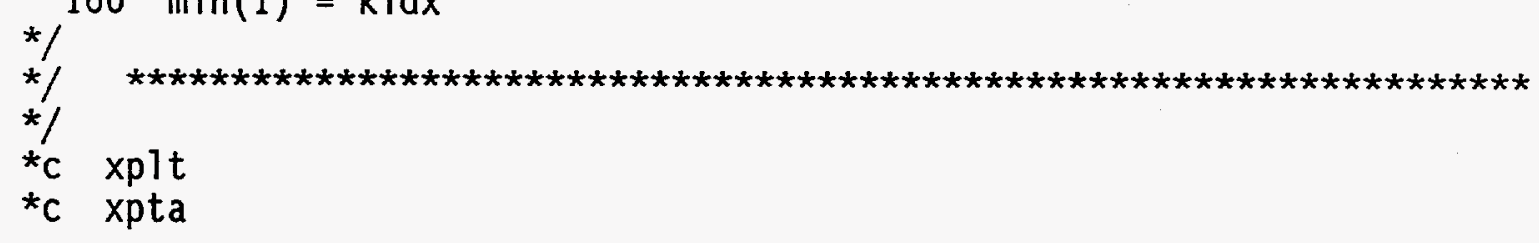




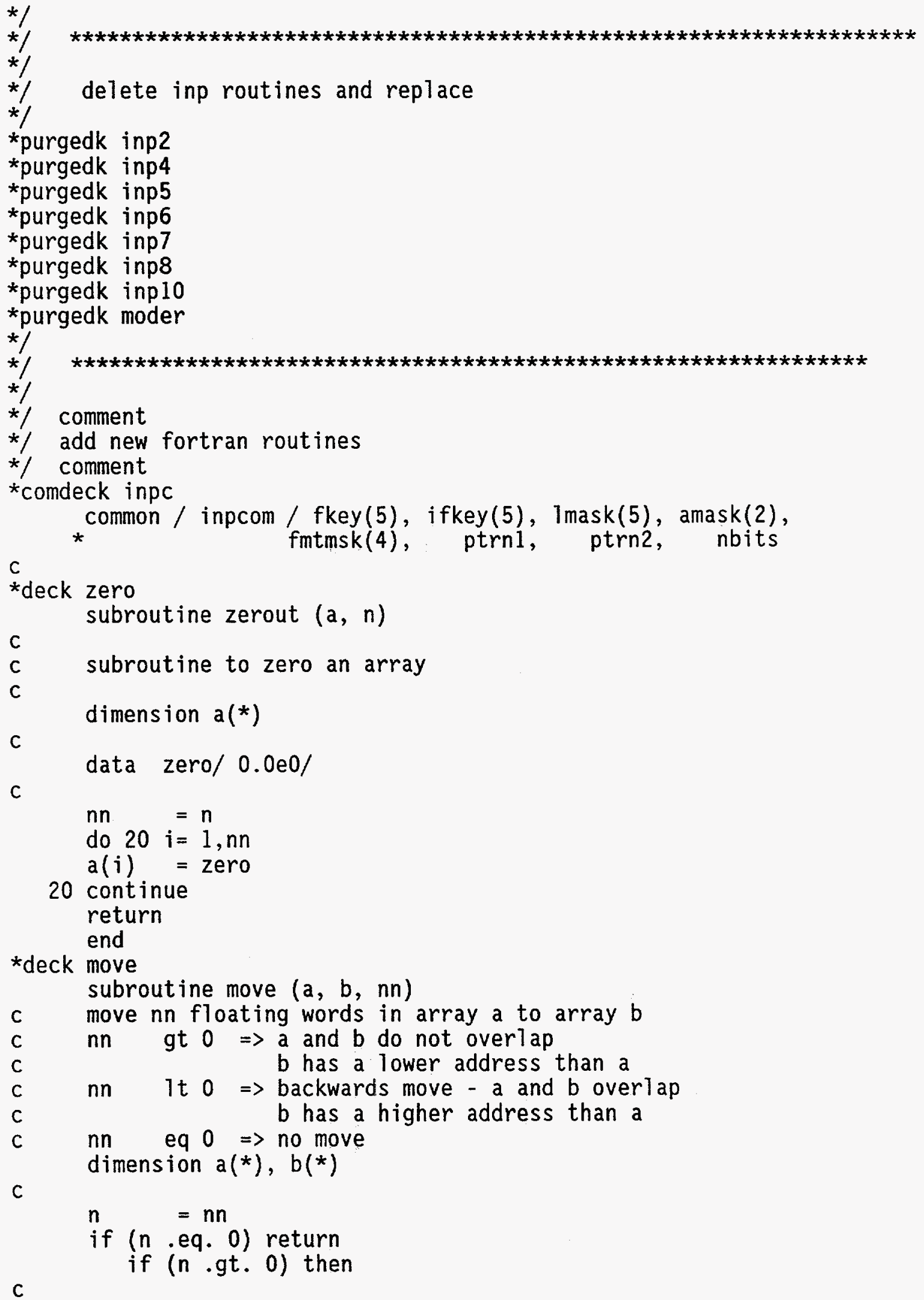


C

C

300

forward move

do $300 i=1, n$

$$
b(i)=a(i)
$$

else

continue

C

C

375

backward move

$k=-n$

$\mathrm{kk}=\mathrm{k}$

do $375 \mathrm{i}=1, \mathrm{k}$

$b(k k)=a(k k)$

$k k=k k-1$

continue

end if

return

end

*deck movi

subroutine movei $(a, b, n n)$

C

c move $n$ n integer words in array a to array b

$c \quad n n$ gt $0 \Rightarrow a$ and $b$ do not overlap

$c \quad b$ has a lower address than a

c nn 1t $0 \Rightarrow$ backwards move - a and b overlap

$c \quad b$ has a higher address than a

$c$ nn eq $0 \Rightarrow$ no move

$\mathrm{C}$

integer $a(*), b(*)$

C

$$
\begin{aligned}
& n=n n \\
& \text { if }(n . e q .0) \text { return } \\
& \text { if }(n . g t .0) \text { then }
\end{aligned}
$$

$\mathrm{C}$

forward move

c

300

$$
\begin{aligned}
& \text { do } 300 i=1, n \\
& b(i)=a(i)
\end{aligned}
$$

else

C

C

C

$$
\text { backward move }
$$

$\begin{array}{ll}\mathrm{k} & =-\mathrm{n} \\ \mathrm{kk} & =\mathrm{k}\end{array}$

do $375 i=1, k$

$b(k k)=a(k k)$

375

$$
\mathrm{kk}=\mathrm{kk}-1
$$

end if

return

end 
*deck ds 1

real function dsl ( $v v, n$ )

C

vv shifted left $n$ bits (arithmetic - circular)

ds $]=\operatorname{shift}(\mathrm{vv}, \mathrm{n})$

return

end

*deck dsr

real function dsr ( $v v, n$ )

c vv shifted right arithmetically $n$ bits

temp = shiftr ( $v v, n$ )

if ( $v v .1 t .0 .0 \mathrm{e} 0)$ then

temp $=$ temp .or. $\operatorname{mask}(n)$

end if

dsr $=$ temp

return

end

*deck dsr 1

real function dsrl ( $v v, n$ )

c vv shifted right logically $n$ bits

dsrl = shiftr (vv, n)

return

end

*deck dor

real function dor ( $v v$, ww)

$c \quad$ logical sum of $v v$ and $w W$

dor $=v v$.or.wW

return

end

*deck dand

real function dand ( $v v$, ww)

c logical product of $v v$ and $W W$

dand $=$ vv. and. $w w$

return

end

*deck isl

integer function isl (kv, n)

c kv shifted left $n$ bits (arithnetic - circular)

isl $=\operatorname{shift}(k v, n)$

return

end

*deck isr

integer function isr ( $k v, n)$

c kv shifted right $n$ bits (arithmetic)

ktem $=\operatorname{shiftr}(k v, n)$

if $(k v .1 t$. o) then

ktem $=$ ktem .or. $\operatorname{mask}(n)$

end if

isr $=$ ktem

return

end

*deck isr 1

integer function isrl (kv, n) 
c kv shifted right logically $n$ bits

isrl = shiftr $(k v, n)$

return

end

*deck i and

integer function iand (kv, $n)$

$c \quad l o g i c a l$ product of $k v$ and $n$

iand $=k v$. and. $n$

return

end

*deck ior

integer function ior ( $k v, n)$

c logical sum of $k v$ and $n$

ior $=k v$.or.n

return

end

*deck locf

integer function locfx(k)

C

C function to return address of variable $n$

c

locfx $=10 c(k)$

return

end

* deck mskf

real function maskf $(n)$

c build a mask of $n$ bits

$c \quad n>0$ mask in high order bits

c $n<0$ mask in low order bits

c

data zero $/ 0.0 \mathrm{e} 0 /$

C

maskf = zero

if $(n . g t .0)$ then

$\operatorname{maskf}=\operatorname{mask}(n)$

else

maskf $=\operatorname{mask}(128+n)$

end if

return

end

*deck cpar

subroutine compar ( $a, b$, nchar)

c compare nchar characters in strings a and b

c nchar $=0$ returned if a mismatch is found

C

character*1 $a(*), b(*)$

nn

$=$ nchar

nchar $=0$

do $20 i=1, n n$

if $(a(i)$.ne. $b(i))$ go to 30

20 continue

nchar $=\mathrm{nn}$ 
30 return

end

*deck pack

subroutine pack (word, noitms, nobits, items)

C

c routine to pack noitms into word from right to left

c nobits $=$ field width in bits for each item

$c$ items = positive integer list to pack

C

integer word

dimension nobits(*), items (*)

C

nn $\quad=$ noitms

isf $=0$

word $=0$

do $20 \mathrm{i}=1, \mathrm{nn}$

$\mathrm{k} \quad=\operatorname{nobits}(i)$

word = word.or.shiftl (items(i), isf)

isf $=$ isf $+k$

20 continue

return

end

*deck unpk

subroutine unpack (word, noitms, nobits, items)

C

c routine to unpack noitms from word from right to left

c nobits $=$ field width in bits for each item

$\mathrm{c} \quad$ items $=$ positive integer list of unpacked items

C

integer word, wordt

C

dimension nobits $\left({ }^{*}\right)$, items $\left({ }^{*}\right)$

C

nn $\quad=$ noitms

wordt $=$ word

do $20 i=1, n n$

$k=\operatorname{nobits}(i)$

items $(i)=$ wordt . and. mask $(128-k)$

20 cont inue

wordt $=\operatorname{shiftr}$ (wordt, $k$ )

return

end

*deck noti

logical function notim (acpu, aio)

C

c dummy function to determine if $\mathrm{cpu}$ or $\mathrm{i} / \mathrm{o}$ time limits are being

c approached.

C

C acpu = dummy

C aio = dummy

C

notim $=$. false. 
return

end

*deck tims

subroutine timset (time)

C

c subroutine to calculate elapsed cpu time

c $\quad$ time $=$ elapsed time - returned

C

C

entry points timel, timer

C

C

c

timel - inner measure of time, elapsed time since last call to either timset, timel, or timer

C

C

C

C

c

C

timer - outer measure of time, elapsed time since last call to timset or timer

timset - initializes timer and timel

atime $=$ second $(t)$

etime $=0.0$

rtime $=0.0$

return

C

entry timel (time)

$n=0$

go to 20

c

entry timer (time)

C

$n=1$

20 continue

etime $=$ second $(t)$

time = etime - atime

atime = etime

rtime = rtime + time

if ( $n$.eq. 1) then

time $=$ rtime

endif

rtime $=0.0$

c

return

end

*deck ftbs

subroutine ftbset (idolr, ndsks, nfils, idxlo, idxhi, length)

C

c initialize ftb common block

c

*call ftbd

integer ftb

data ftb / $3 \mathrm{hftb} /$

C

if (first .ne. ftb) first $=$ idolr 


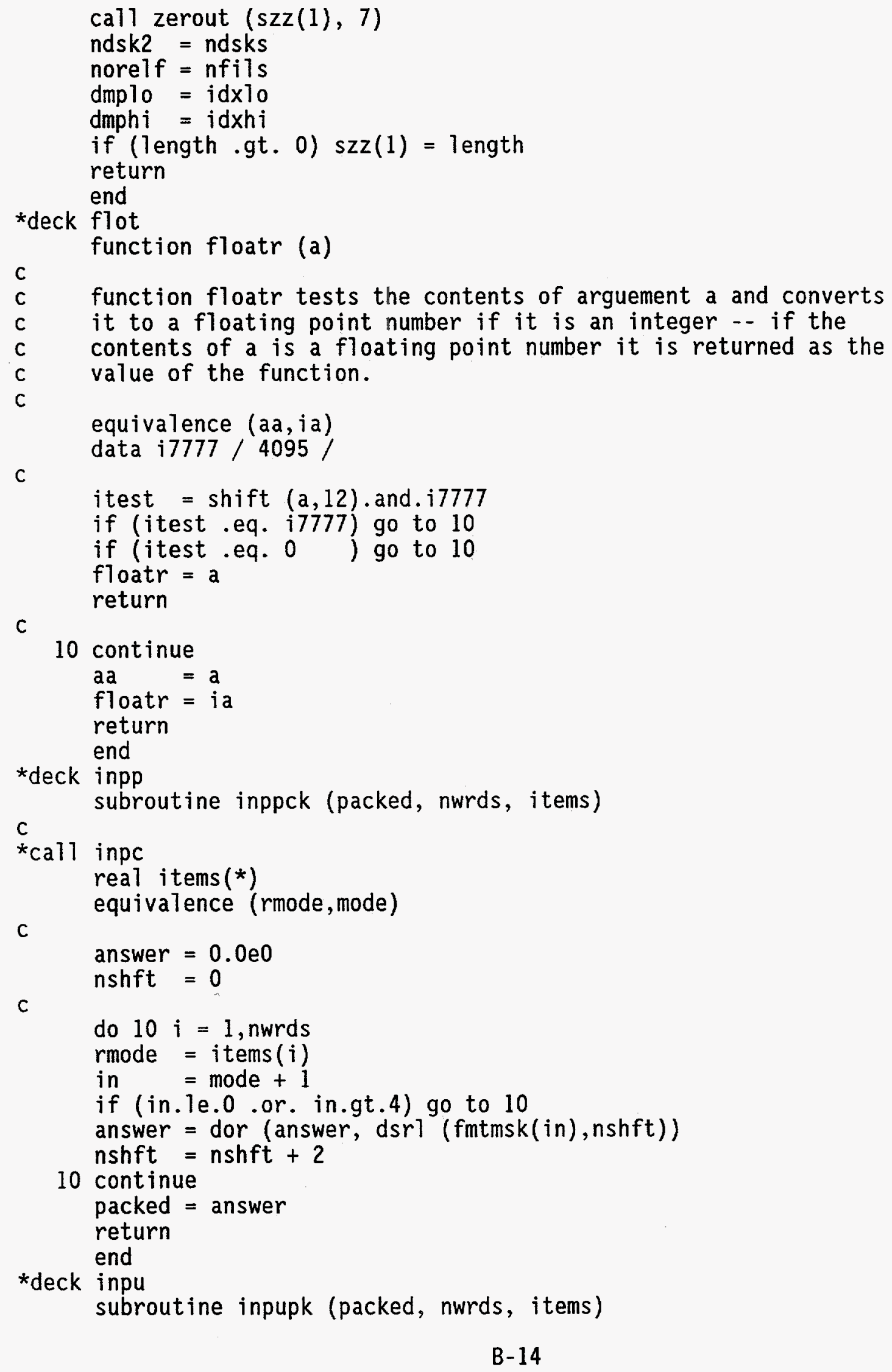




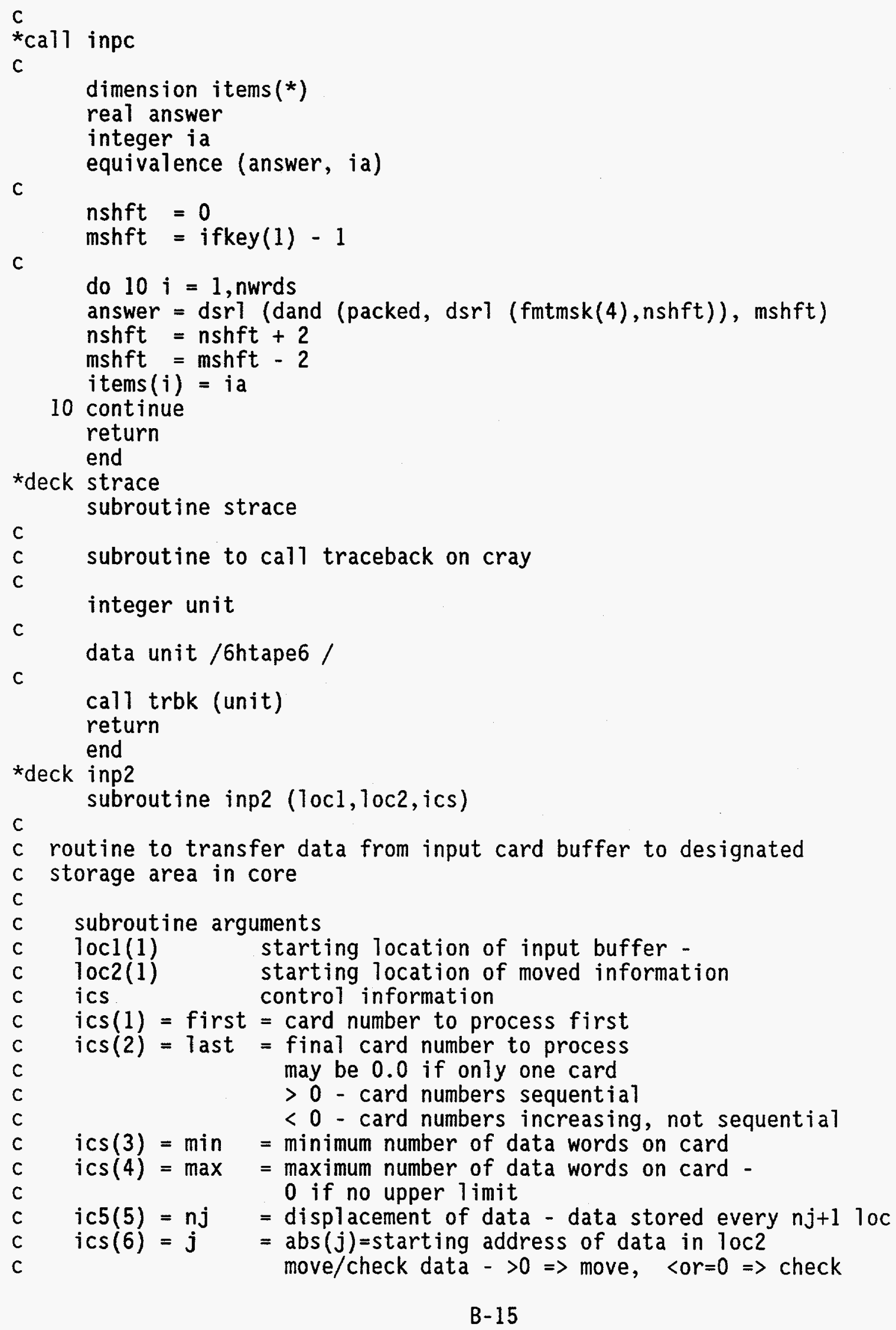




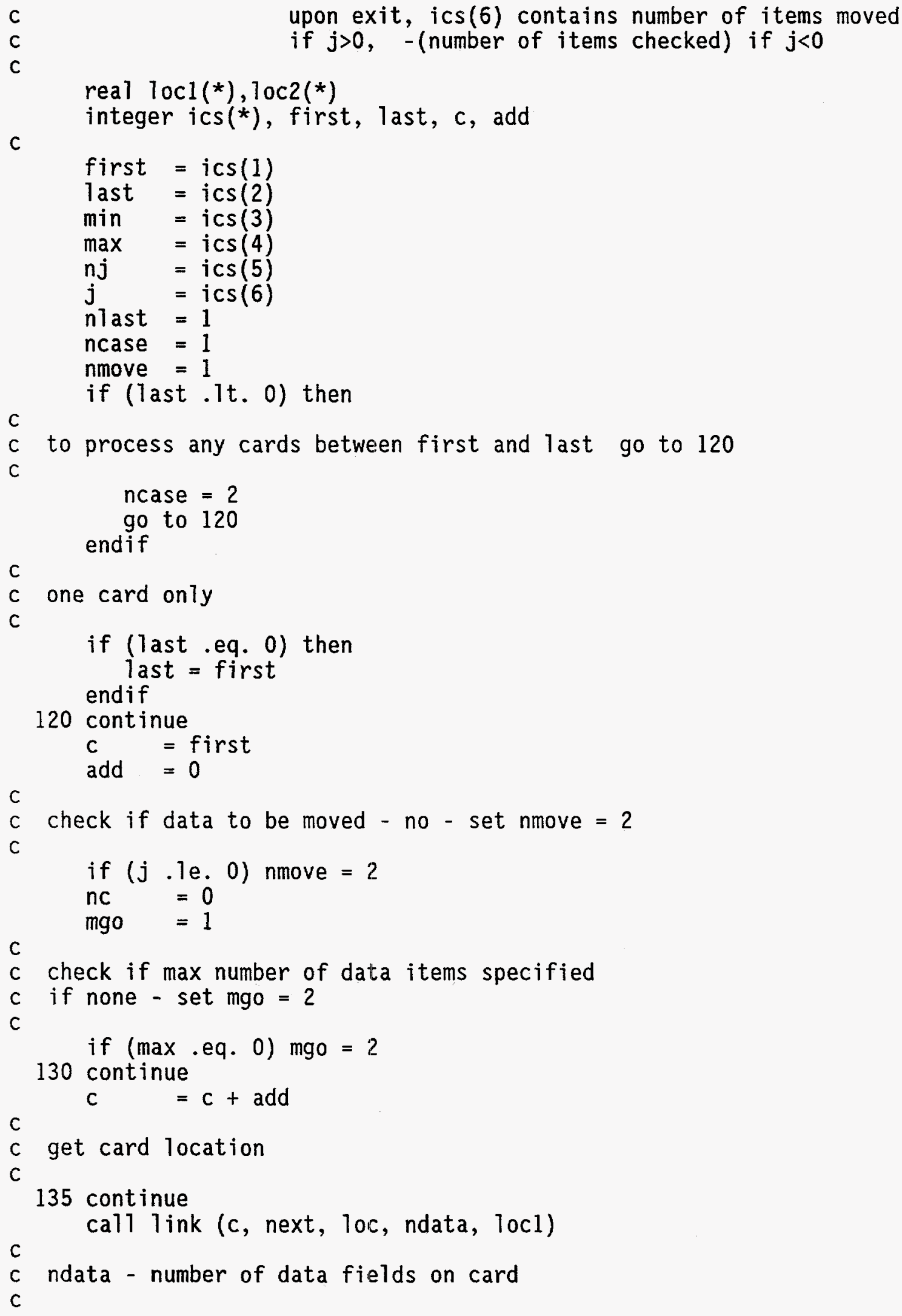




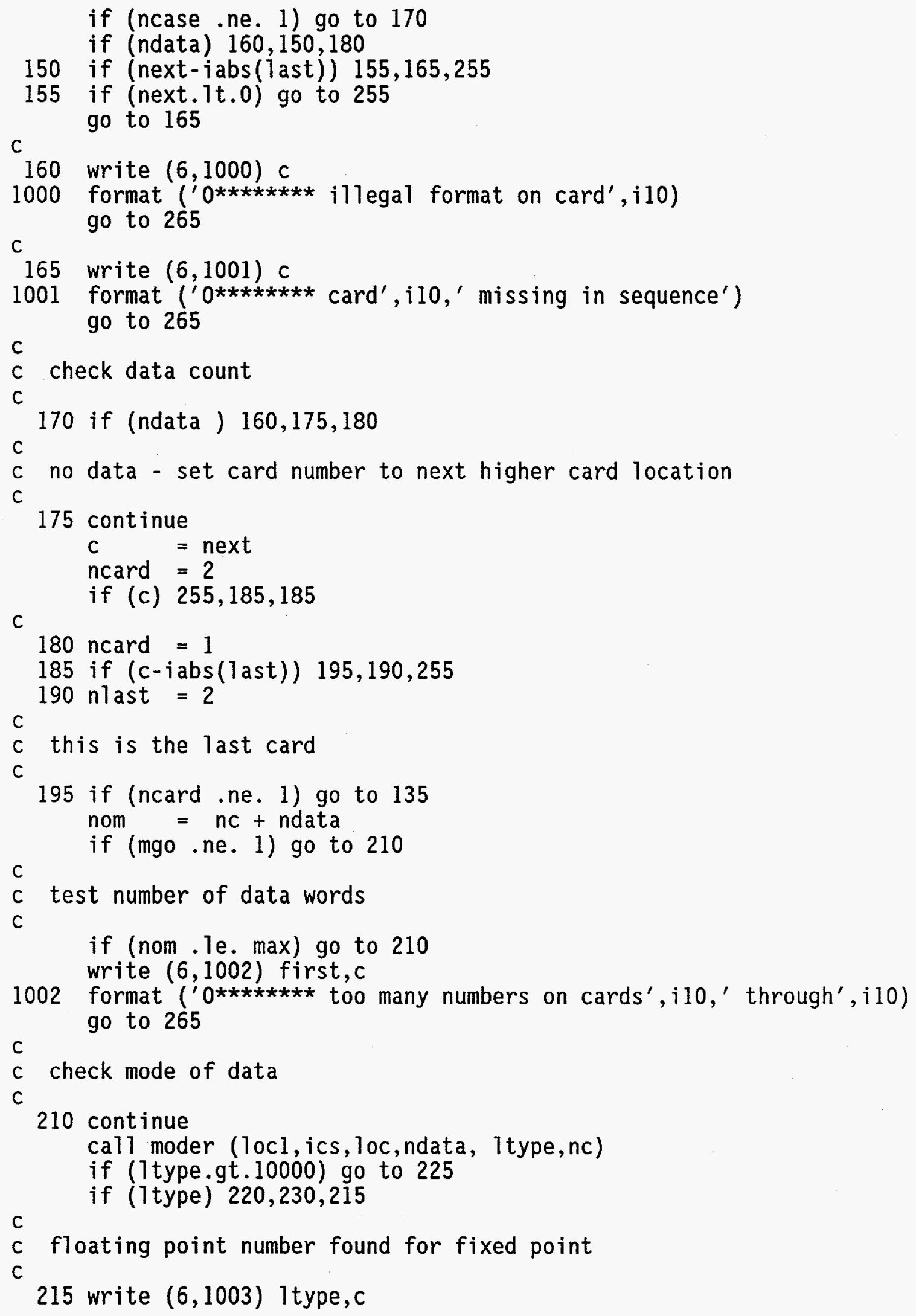


1003 format ('0******** word' $, i 3,^{\prime}$ on $\mathrm{card}^{\prime}, \mathrm{i} 10$, go to 265

C

$c$ fixed point number found for a floating point

c

2201 type $=-7$ type

write $(6,1004) 1$ type,c

1004 format (' $(0 * * * * * * * *$
$*$

go to 265

C

c alpha-numeric data expected - not found

C

225 1type $=1$ type -10000

write $(6,1005)$ 1type, $c$

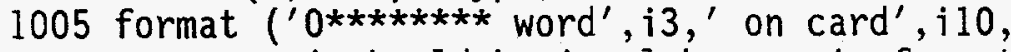

* 'should be in alphanumeric format')

go to 265

c

c check if data to be moved to new location

C

230 continue

if (nmove.ne. 1) go to 245

C

c move to new location

c

$1=10 \mathrm{c}$

maxnum $=1+$ ndata -1

$10 c b=j+(n j+1) \star n c$

C

do 240 loca $=1$, maxnum

$10 \mathrm{c} 2(10 \mathrm{cb})=10 \mathrm{cl}$ (10ca)

240 continue

$10 c b=10 c b+n j+1$

C

245 continue

$n c=n c+$ ndata

c

c check for last card

C

if (nTast .ne. 1) go to 255

c

c another card expected

c

add $=1$

go to 130

c

c last card

c

255 continue

if (nc .ge. min) go to 270 


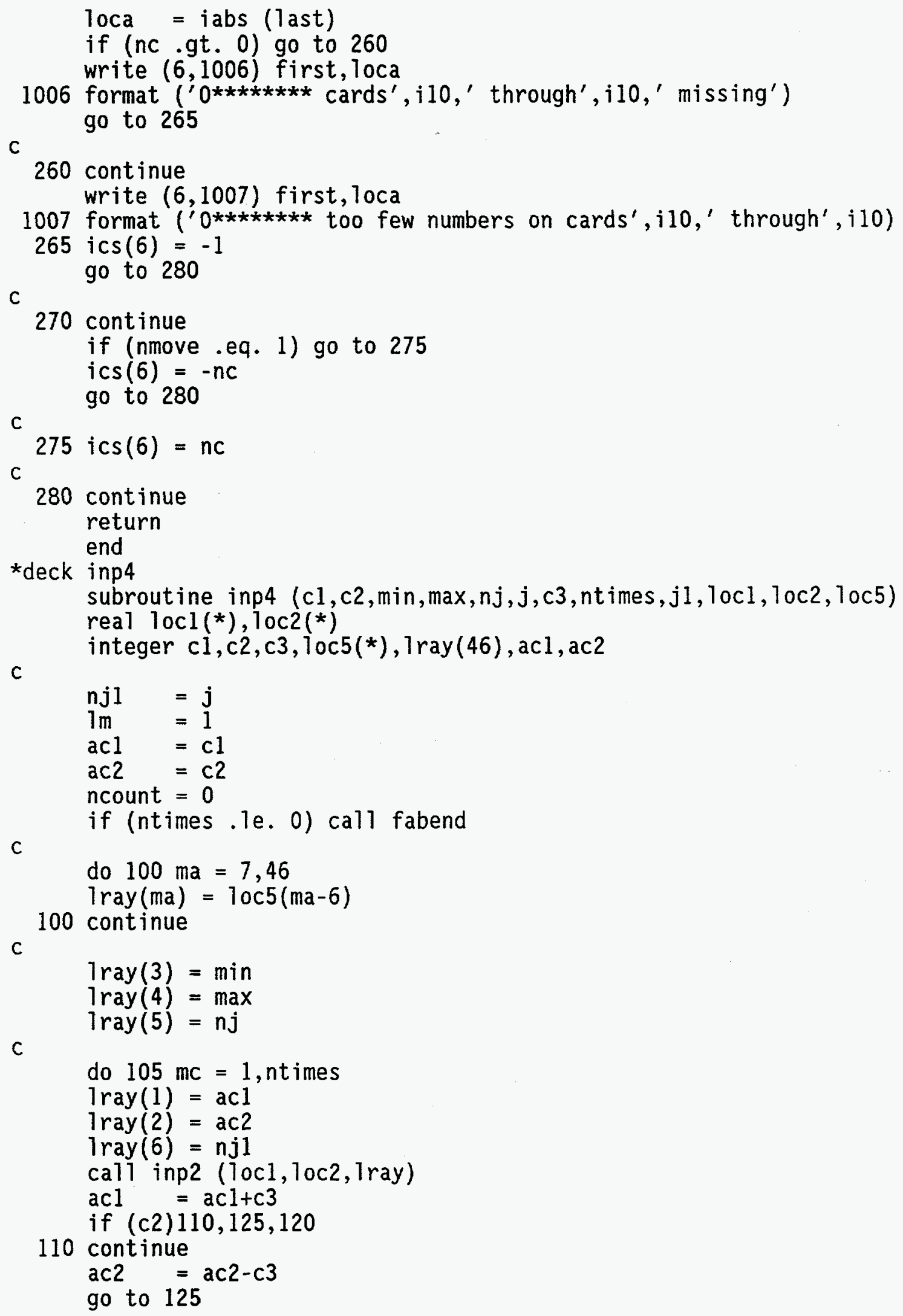




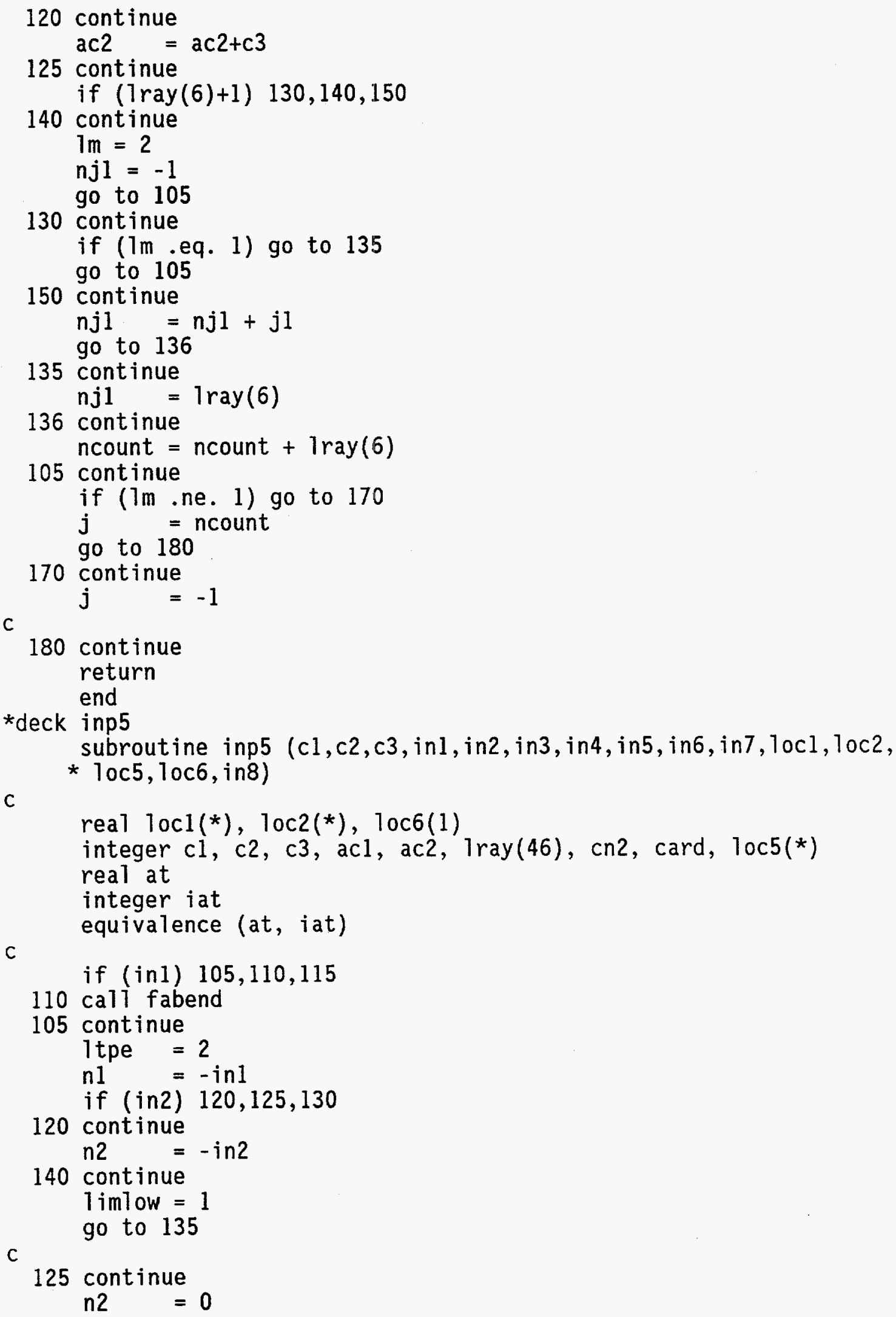




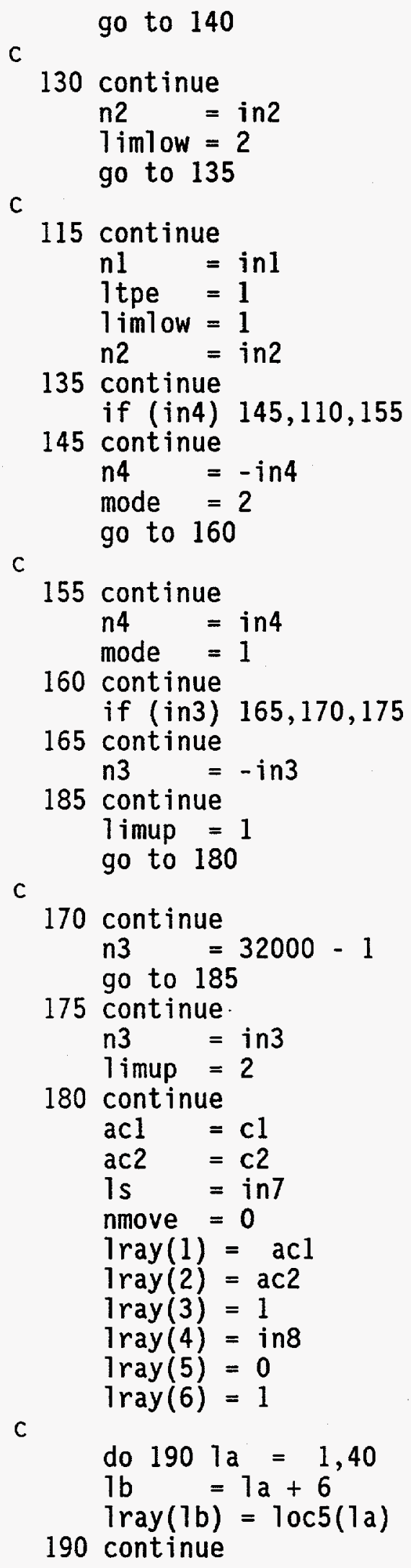


C

do $100 \mathrm{mad}=1$, in 5

cal1 inp2 (10c1,10c6, 1ray)

nmov $=1$ ray $(6)$

if (nmov) $205,500,210$

205 continue

in $7=-1$

go to 500

210 cont inue

1 up $1 \mathrm{im}=1$ imup

lowl im $=1 \mathrm{imlow}$

nmove $=$ nmove + nmov

$\mathrm{kl}=\mathrm{nl}+1 \mathrm{tpe}$

$\mathrm{k} 2=\mathrm{nmov} / \mathrm{k} 1$

last $=\mathrm{n} 2$

if (nmov .eq. $k 1 * k 2$ ) go to 220

$\mathrm{cn2}=\operatorname{iabs}(\mathrm{ac} 2)$

write $(6,1)$ nmov, acl, cn2, k1

1 format ('0******** ', $i 5$, ' numbers on cards', $i 10$,' through', i10,

* $\quad$ are not a multiple of ${ }^{\prime}$, i4)

in7 $=-1$

go to 500

C

220 continue

if (mode .ne. 1) go to 230

$k 4=1 s+n 4 * 1$ ast

$\mathrm{k} 5=1$

$\mathrm{k} 6=\mathrm{n} 4$

go to 235

c

230 continue

$\mathrm{k} 4=1 \mathrm{~s}+1 \mathrm{ast}$

$k 5=n 4$

$k 6=1$

235 continue

C

do $400 \mathrm{k} 3=1$, nmov, $\mathrm{k} 1$

if (1tpe .ne. 1) go to 245

nstart $=1$ ast +1

$\mathrm{k} 12=\mathrm{k} 3$

$k 13=k 3+n 1-1$

go to 250

245 continue

at $=10 \mathrm{c} 6(\mathrm{k} 3)$

nstart $=$ iat

$k 12=k 3+1$

$k 13=k 3+n 1$

if (nstart-n2) 255,260,265

$255 \mathrm{cn} 2=$ iabs (ac2)

cal1 inp6 (ac1,cn2, k3, card, item, 1oc1)

write $(6,2)$ item, card, $n 2$

2 format ('0******** item', $i 3$,' on card', $i 10$, ' is less than minimum' 


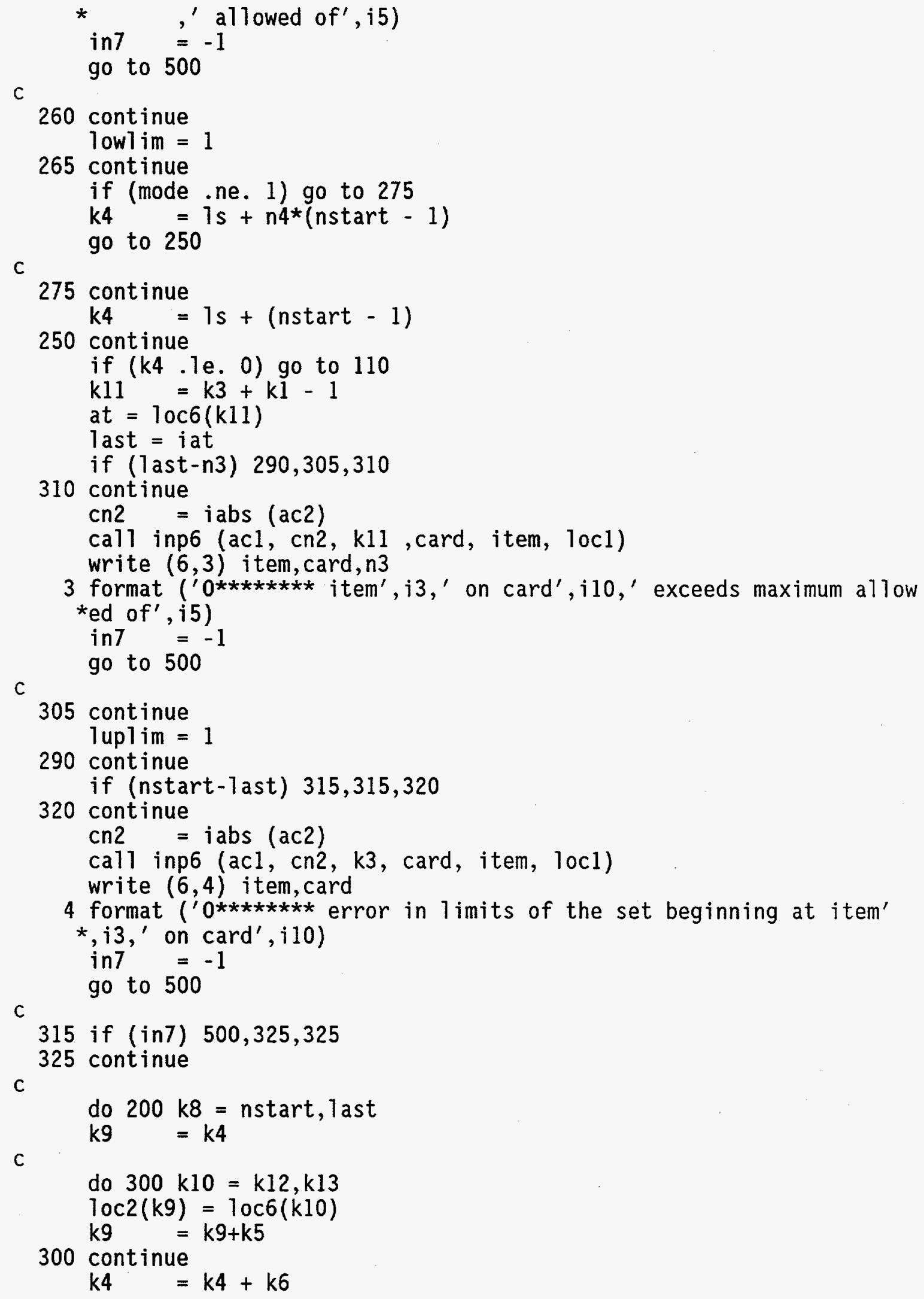




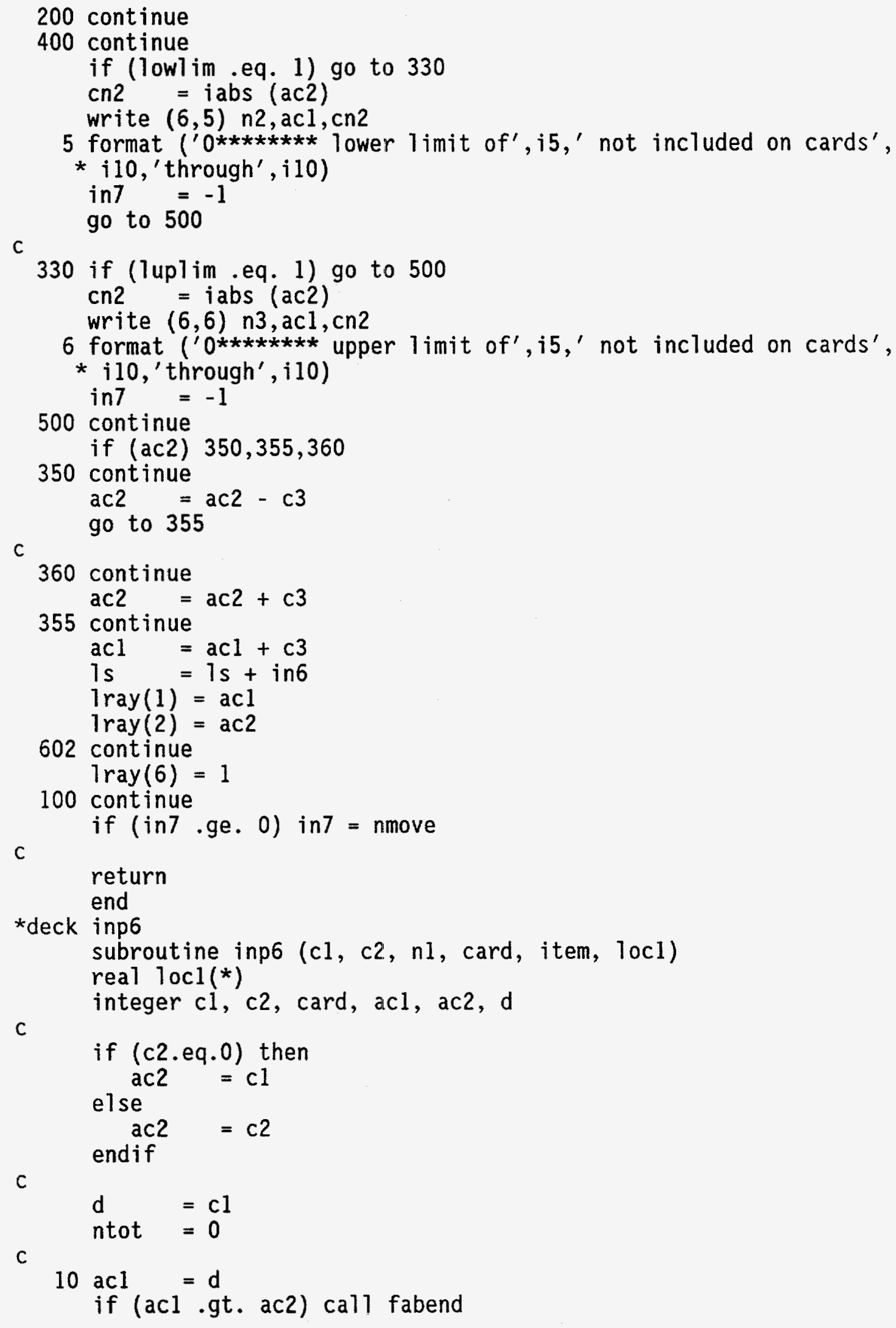

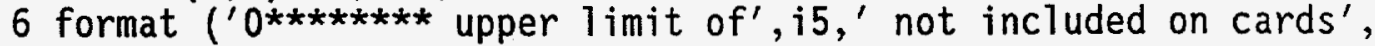

C

return

end

*deck inp6

subroutine inp6 (c1, c2, $n 1$, card, item, 1ocl)

real $10 \mathrm{cl}\left({ }^{*}\right)$

C

integer c1, c2, card, acl, ac2, d

if (c2.eq.0) then

else ac2 $=\mathrm{cl}$

$\mathrm{ac2}=\mathrm{c2}$

end if

c

$\mathrm{d}=\mathrm{cl}$

ntot $=0$

C

$10 \mathrm{acl}=\mathrm{d}$

if (ac1 .gt. ac2) call fabend 
C

call link (acl, d, m, n, locl)

ntot $=$ ntot $+n$

if (ntot .1t. nl) go to 10

card $=\mathrm{acl}$

c

item $=n 1-($ ntot $-n)$

return

end

*deck inp7

subroutine inp7 (card, item)

integer card

C

write $(6,1)$ item, card

1 format ('0******** item', $i 3$, ' on card', $i 10$, ' in error')

return

end

*deck inp8

function inp8 (1ist,a)

C

c inp8 on return is the number of extraneous cards found.

c here extraneous means no reference by link (hence, also inp2,4,5)

c

dimension $a(*)$

real $r l$

integer irl

equivalence $(r 1, i r l)$

C

icnt $=0$

$r 1=a(1)$

$i 1=i r 1+3$

$r 1=a(2)$

$i 2=i 1+i r 1-1$

if ( $i 2$. ge. il) then

c

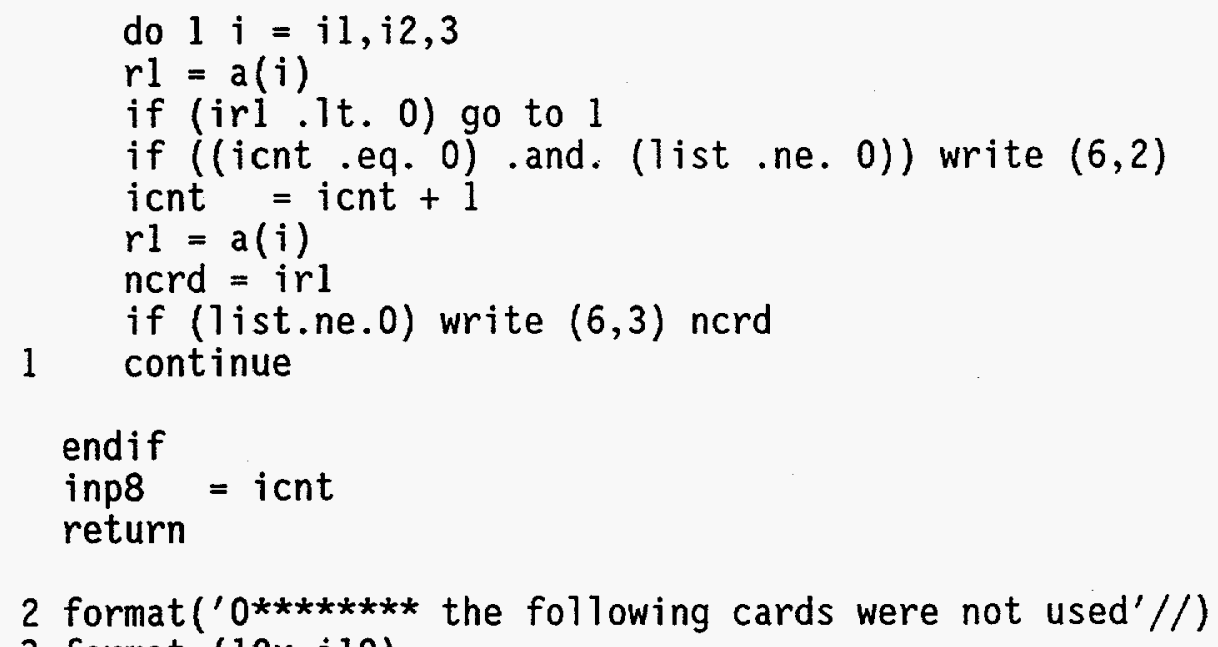

inp8 $=$ icnt

2 format (' $0 * * * * * * * *$ the following cards were not used'//)

C

endif return

c

3 format $(10 x, i 10)$

end 


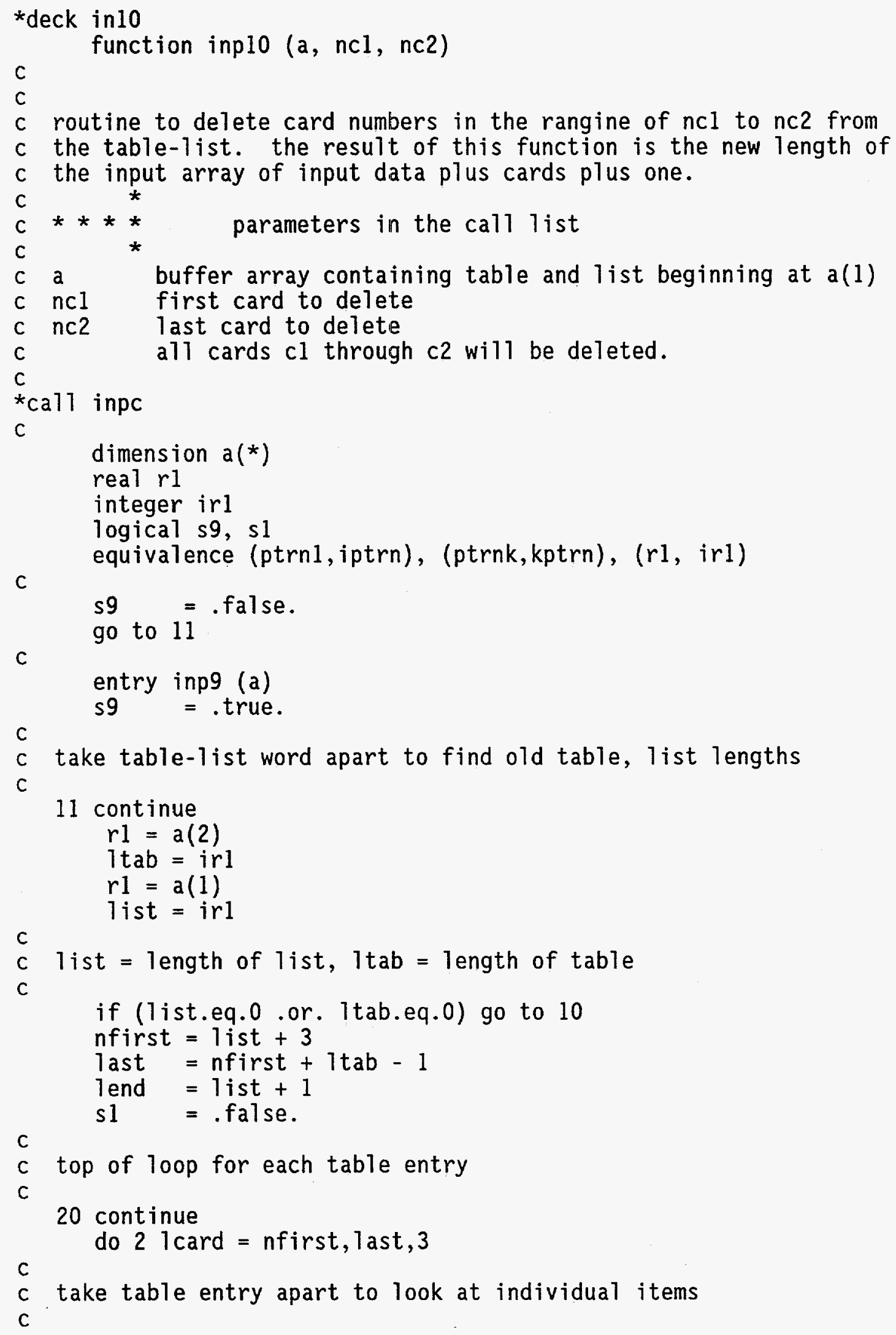




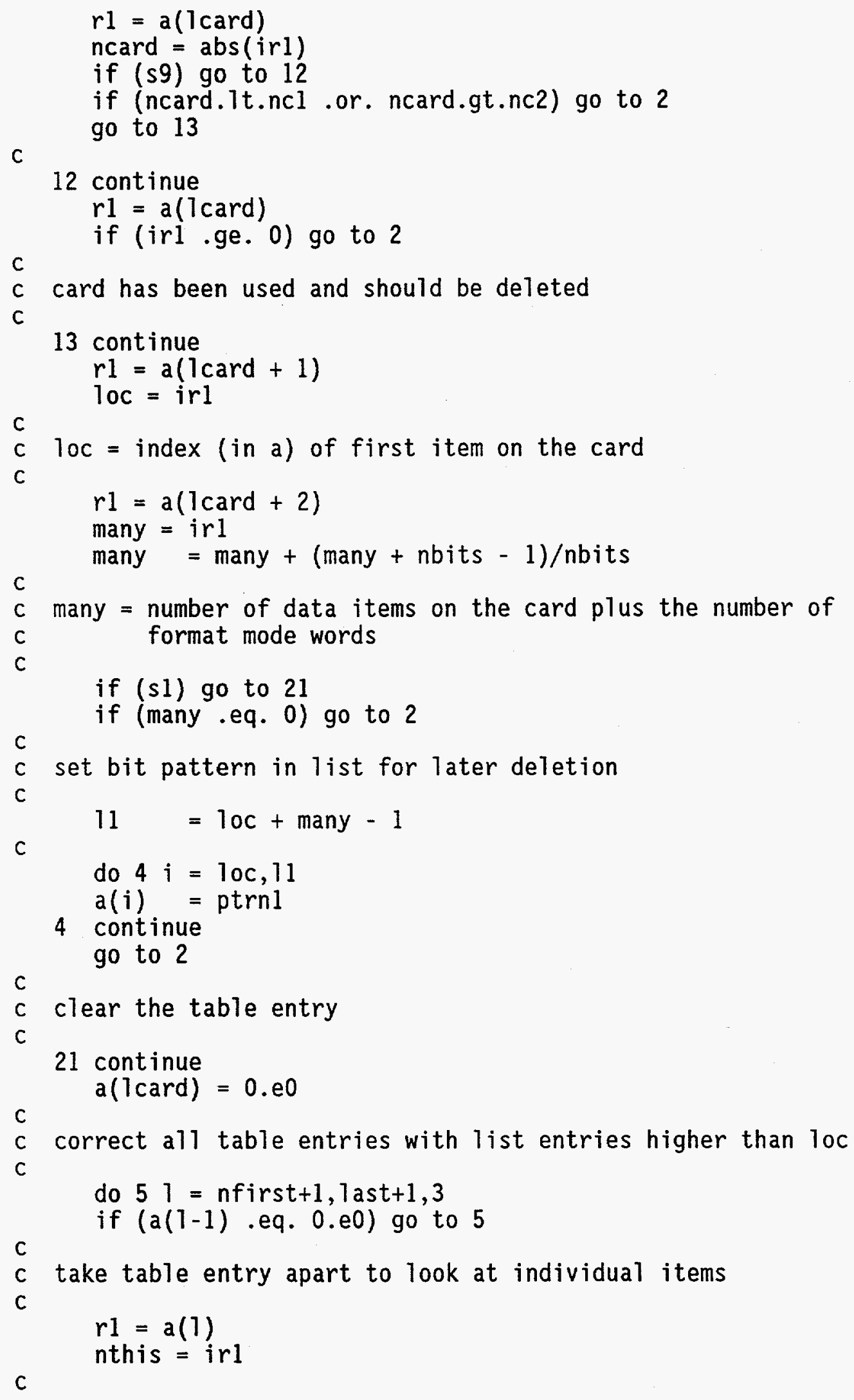




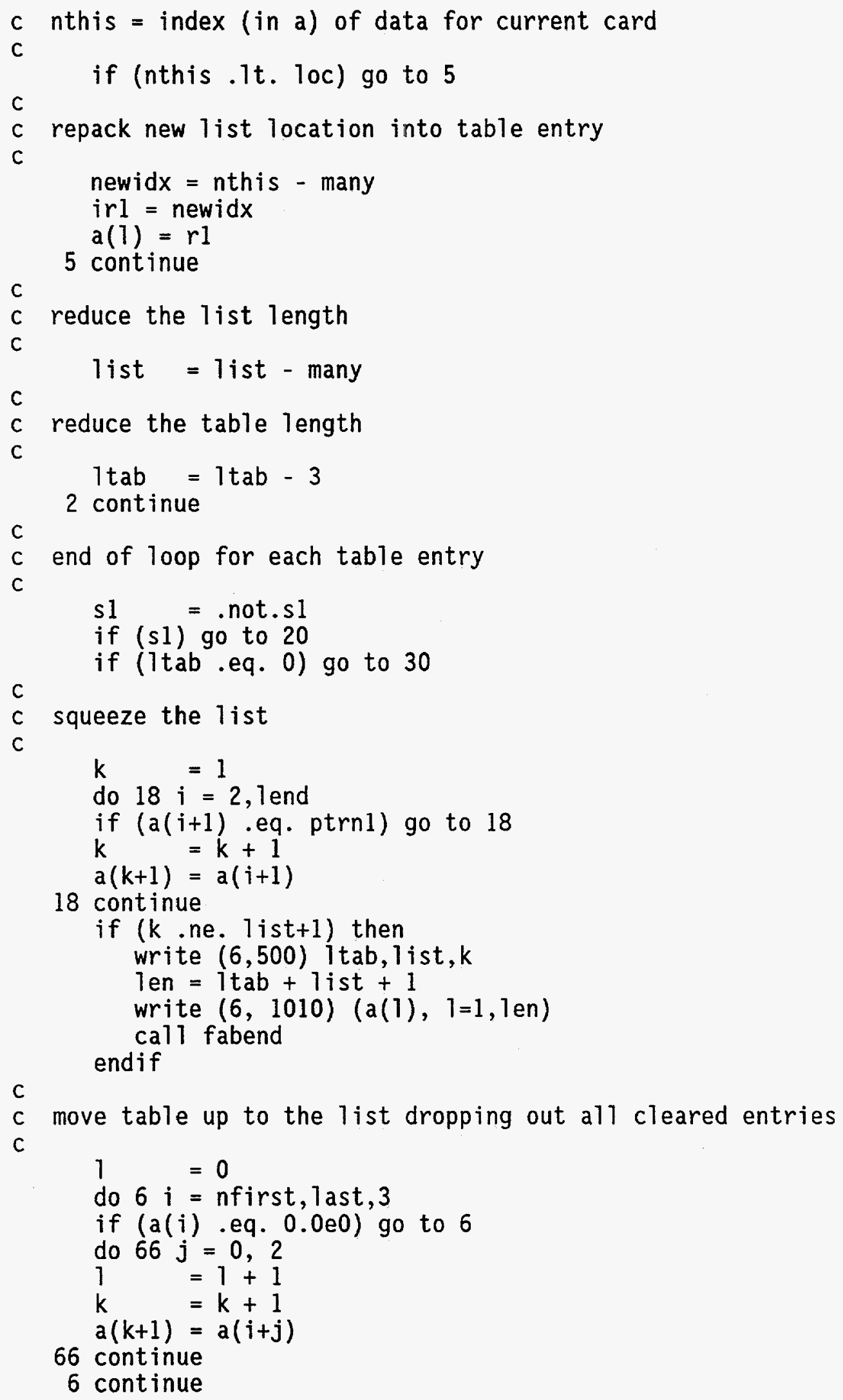




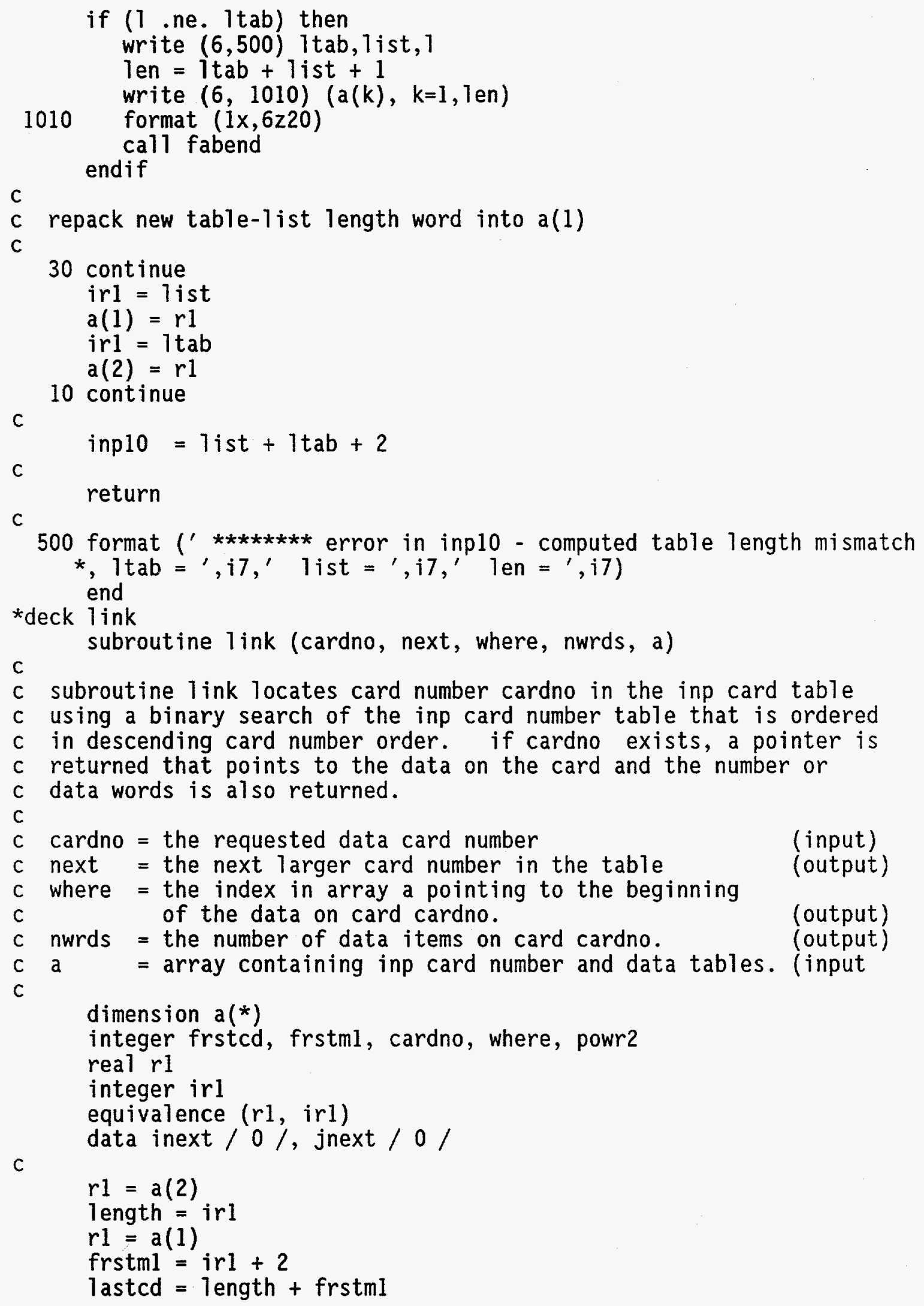




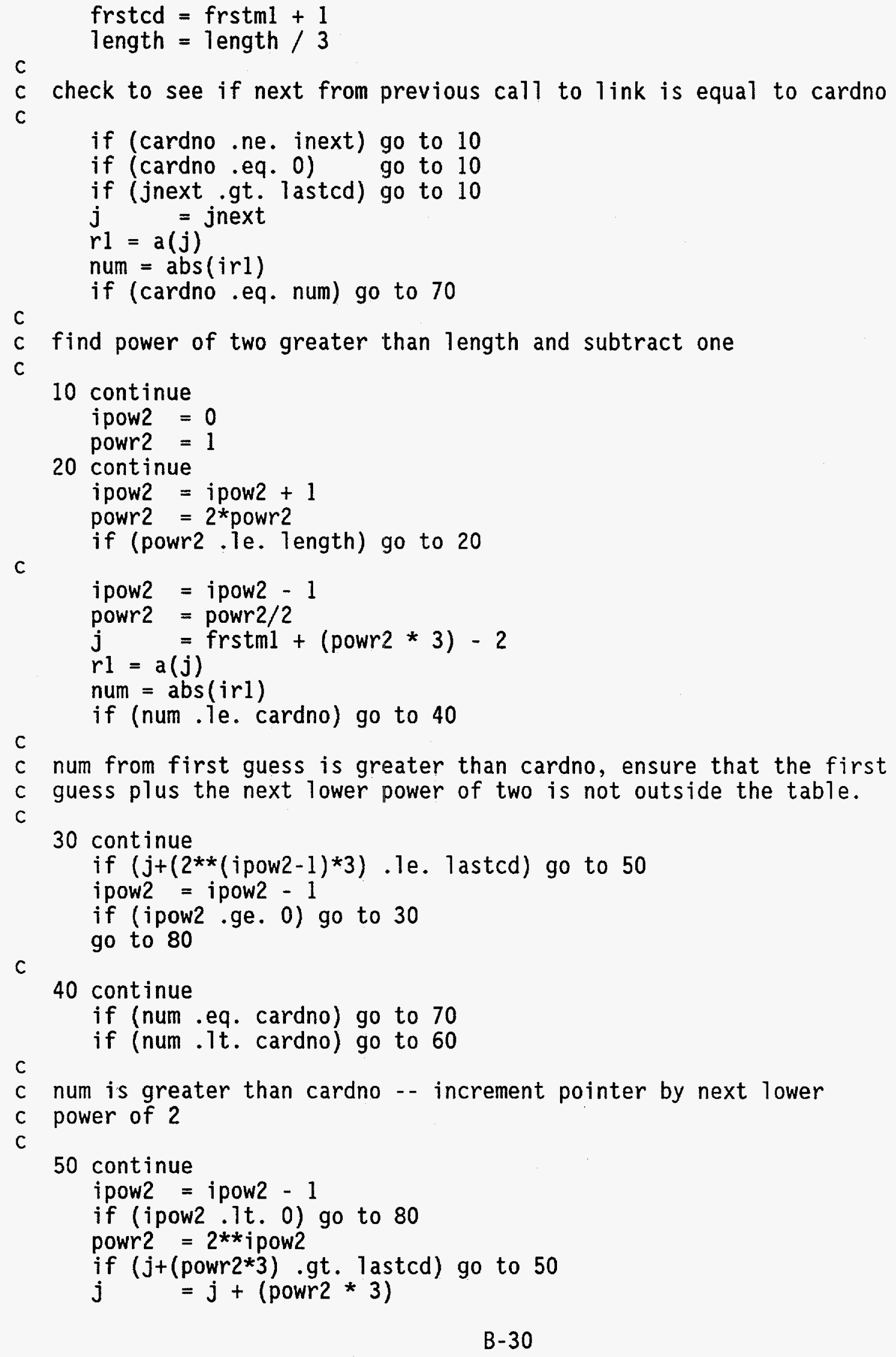




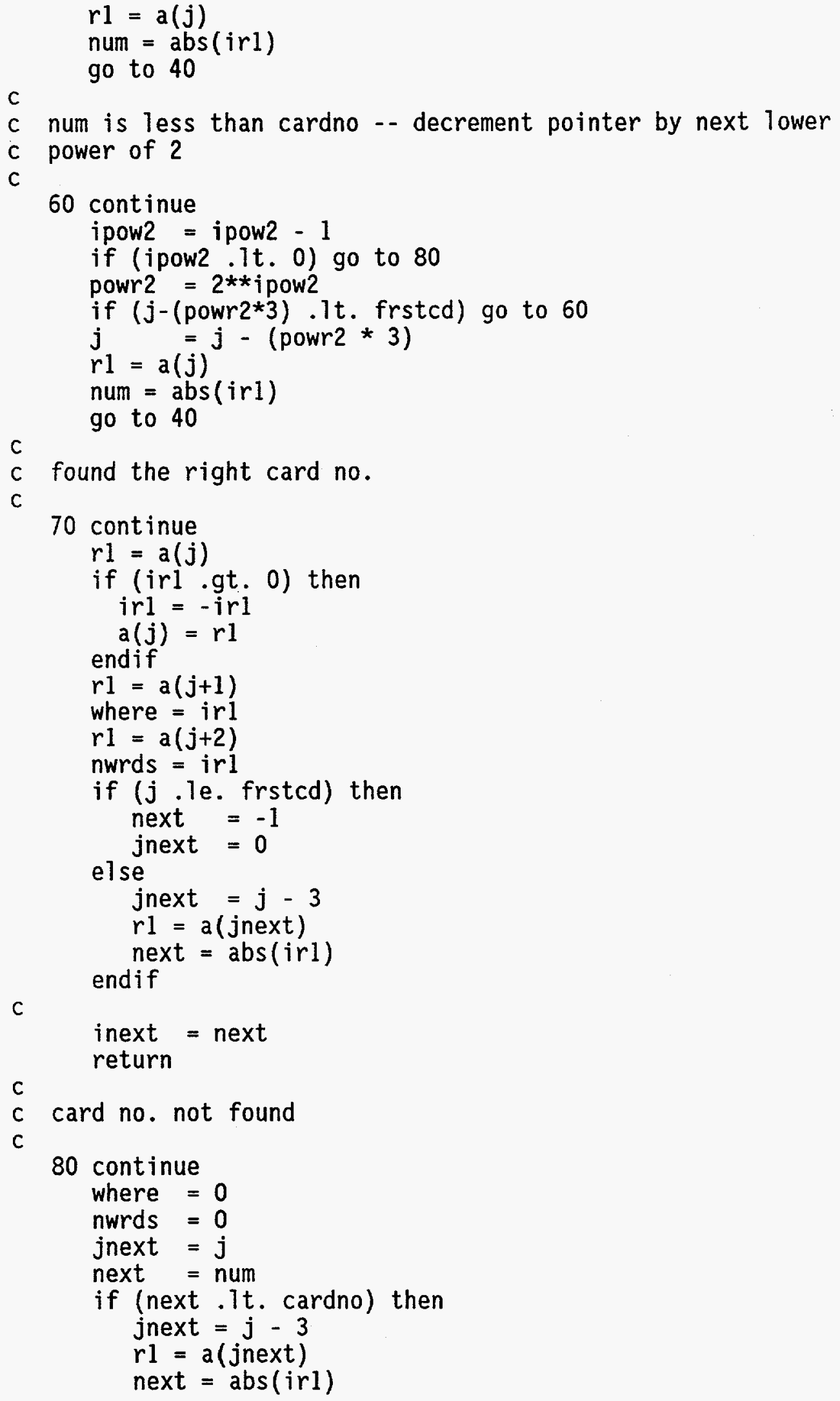




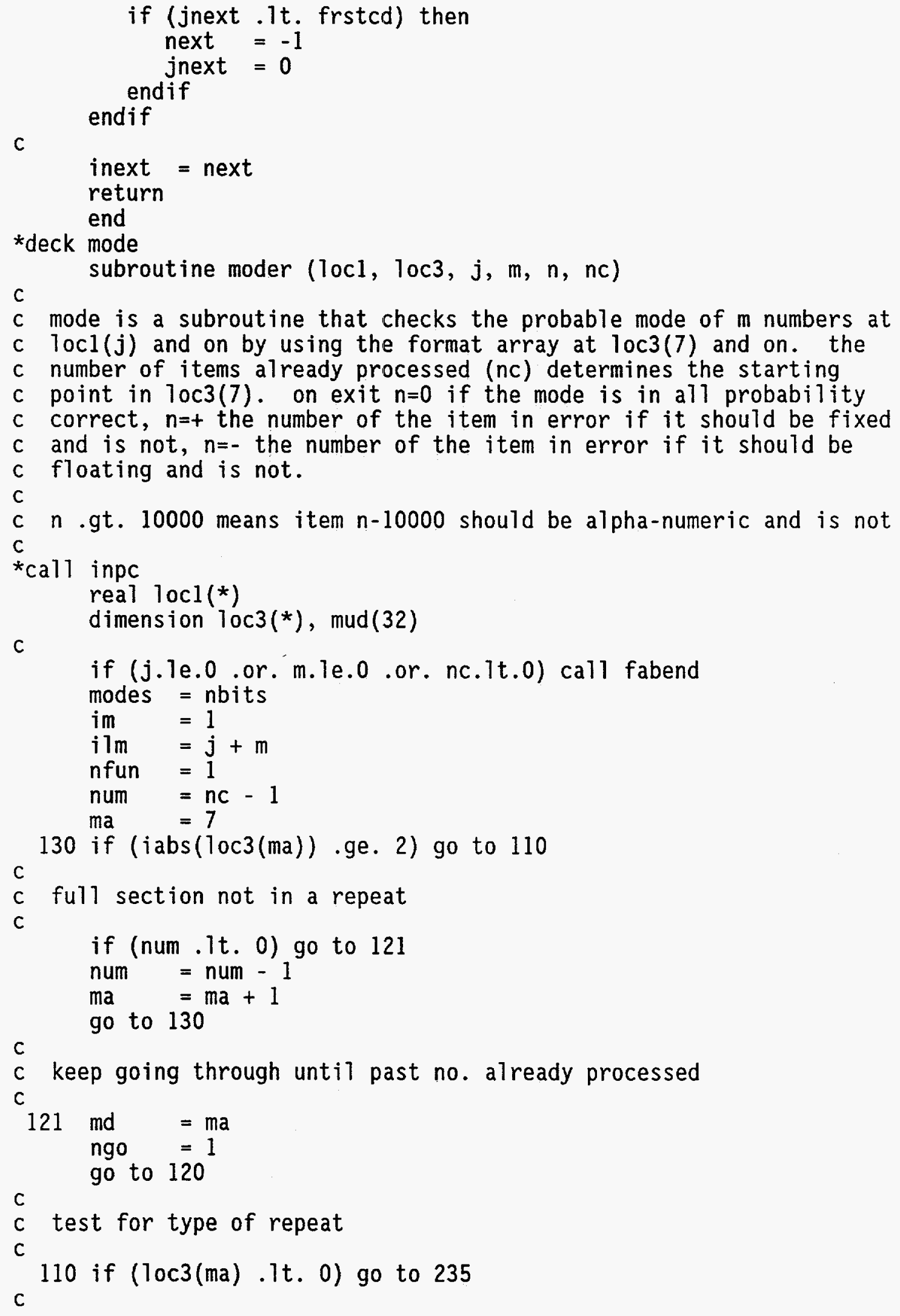




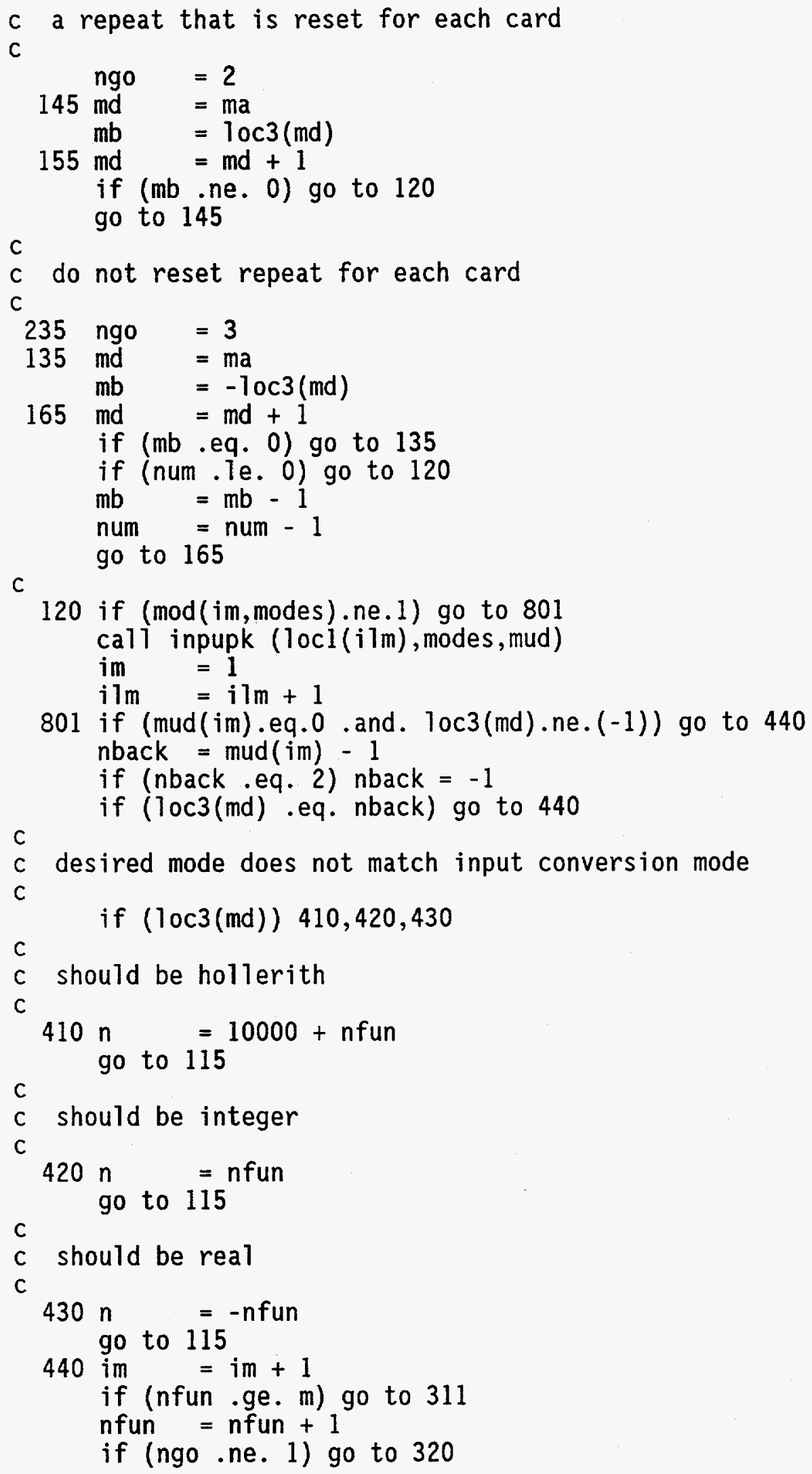




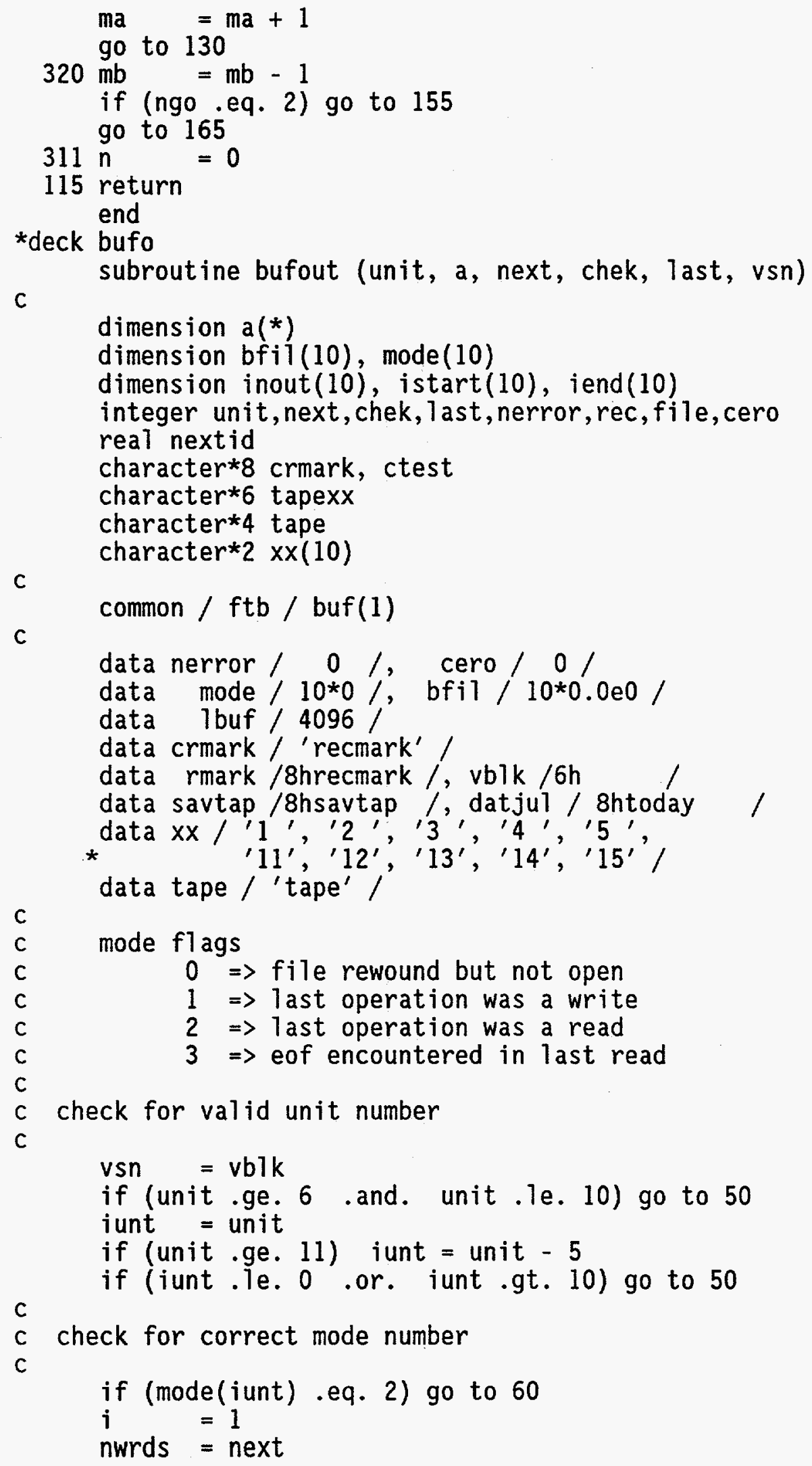


1ast $=$ next

lohi $=$ chek

chek $=1$

C

C open file buffers if mode is 0

C

if (mode(iunt) .eq. 0) then

C tapexx = tape $/ / x x$ (iunt) open (unit=unit, file=tapexx, status='new', access=' sequential',

* form $=$ ' unformatted', err=70, iostat=iostat)

C

bfil (iunt) $=$ nextid $(0)$

call reserv (bfil(iunt), lbuf, lohi, istart(iunt)) inout (iunt) $=$ istart (iunt) endif iend (iunt) = istart (iunt) + 1buf -1

C

mode $($ iunt $)=1$

c

20 continue

$\min =\operatorname{inout}$ (iunt)

$\max =\min +$ nwrds -1

if $(\max . g e$. iend(iunt)) go to 30

C

do $25 \mathrm{j}=\min , \max$

$\operatorname{buf}(j)=a(i)$

$i=i+1$

25 continue

inout (iunt) $=\max +1$

return

C

C write spans a buffer - fill rest of buffer then write

c

30 continue

$\max =$ iend (iunt)

C

do $35 \mathrm{j}=\min , \max$

$\operatorname{buf}(j)=a(i)$

$i=i+1$

35 continue

c

i10 $\quad$ istart (iunt)

ihi = iend (iunt)

write (unit,err $=40$, iostat=iostat) (buf(1), $1=i 10, \max$ )

nwrds $=$ nwrds + min $-\max -1$

inout (iunt) $=$ istart (iunt)

if (nwrds .gt. 0) go to 20 return

C

c write error has occurred

c 


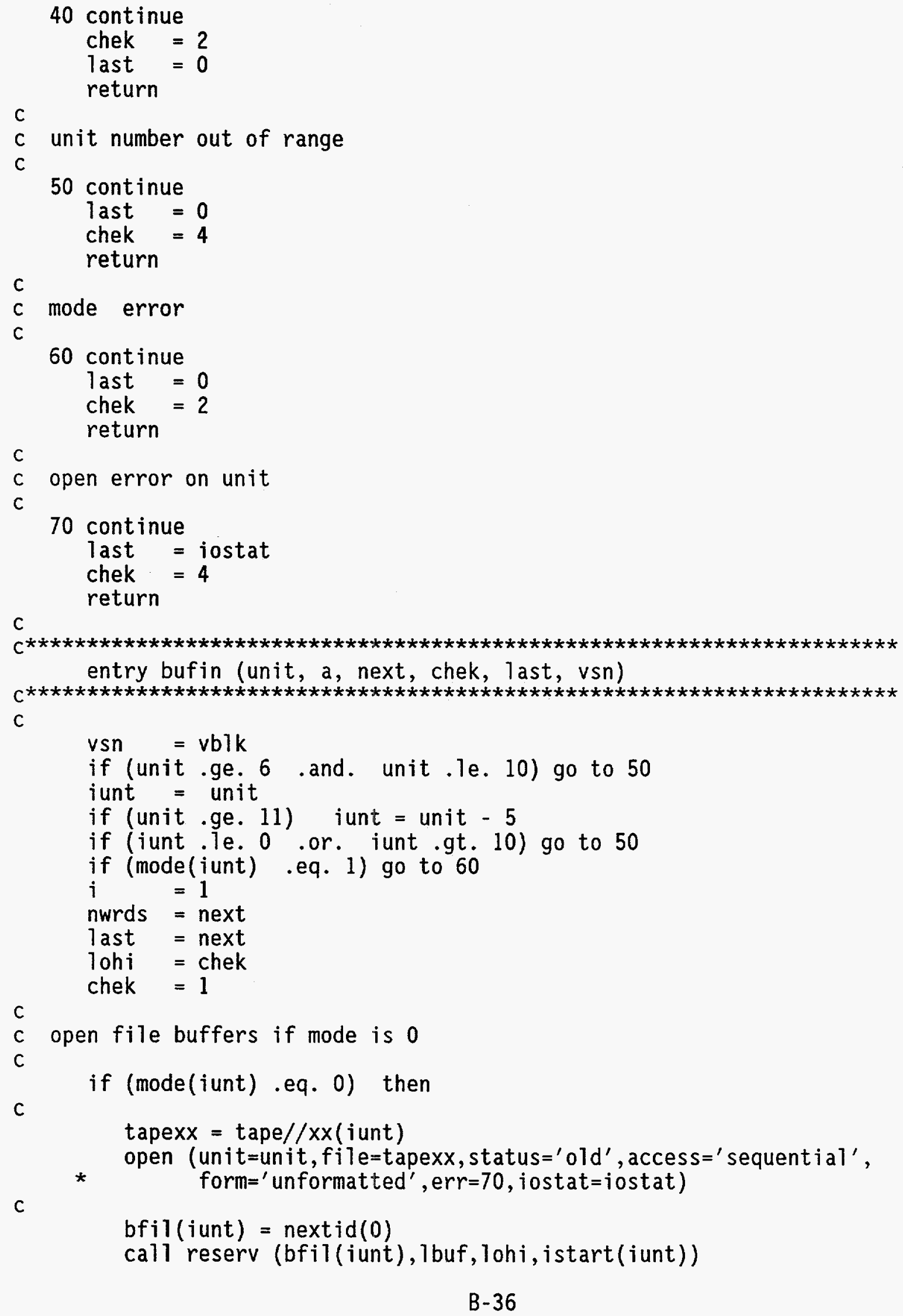




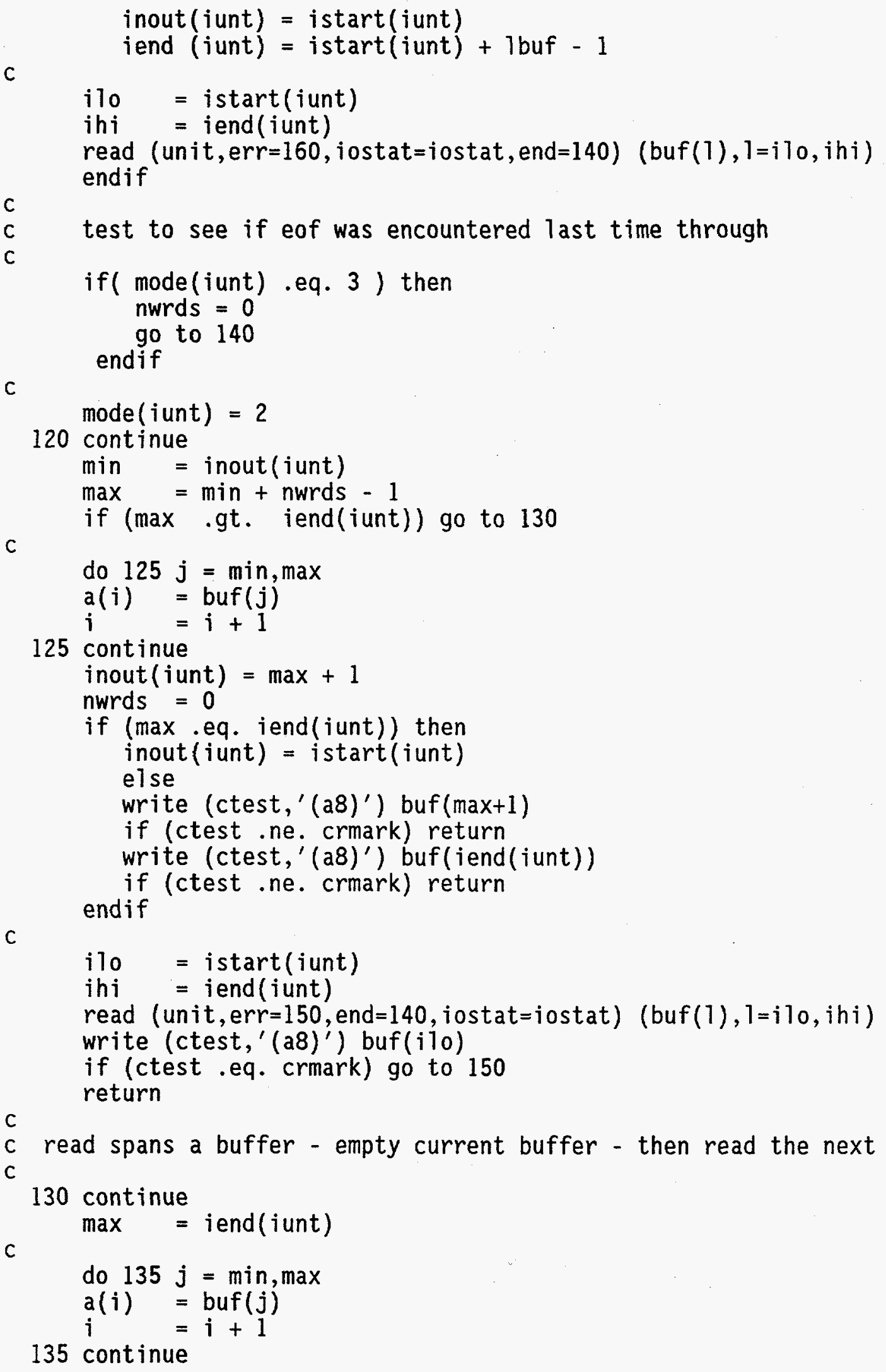


C

i10 = istart(iunt)

ihi = iend (iunt)

read (unit, err $=150$, end $=140$, iostat=iostat) (buf(1), $1=i 10$, ihi)

write (ctest,' (a8)') buf(i10)

if (ctest .eq. crmark) go to 150

nwrds $=$ nwrds $+\min -\max -1$

inout (iunt) $=$ istart (iunt)

if (nwrds .gt. 0) go to 120

return

C

C end of file encountered

C

140 continue

mode (iunt) $=3$

chek $=3$

last $=$ next - nwrds

return

c read error - if a record mark, read next buffer

C

150 continue

c check if read error was a short read

write (ctest,' (a8)') buf(istart(iunt))

if (ctest. ne. crmark) go to 40

write (ctest,' (a8)') buf(iend(iunt))

if (ctest.ne. crmark) go to 40

C

i10 = istart (iunt)

ihi = iend (iunt)

read (unit, err $=160$, end $=140$, iostat=iostat) (buf (1), $1=i 10$, ihi)

mode (iunt) $=2$

inout (iunt) $=$ istart (iunt)

if (nwrds.gt. 0) go to 120

return

C

c read error -

c

160 cont inue

chek $=2$

last $=0$

return

C

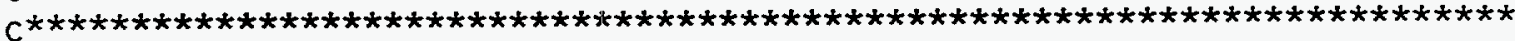

entry bufskp (unit, rec, file, chek)

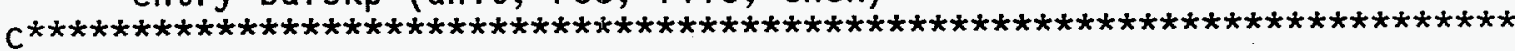

C

if (unit.ge. 6 .and. unit.1e. 10) go to 50

iunt = unit

if (unit.ge. 11) iunt = unit - 5

if (iunt . le. 0 .or. iunt.gt. 10) go to 50

if (file.1t. 0) go to 330 


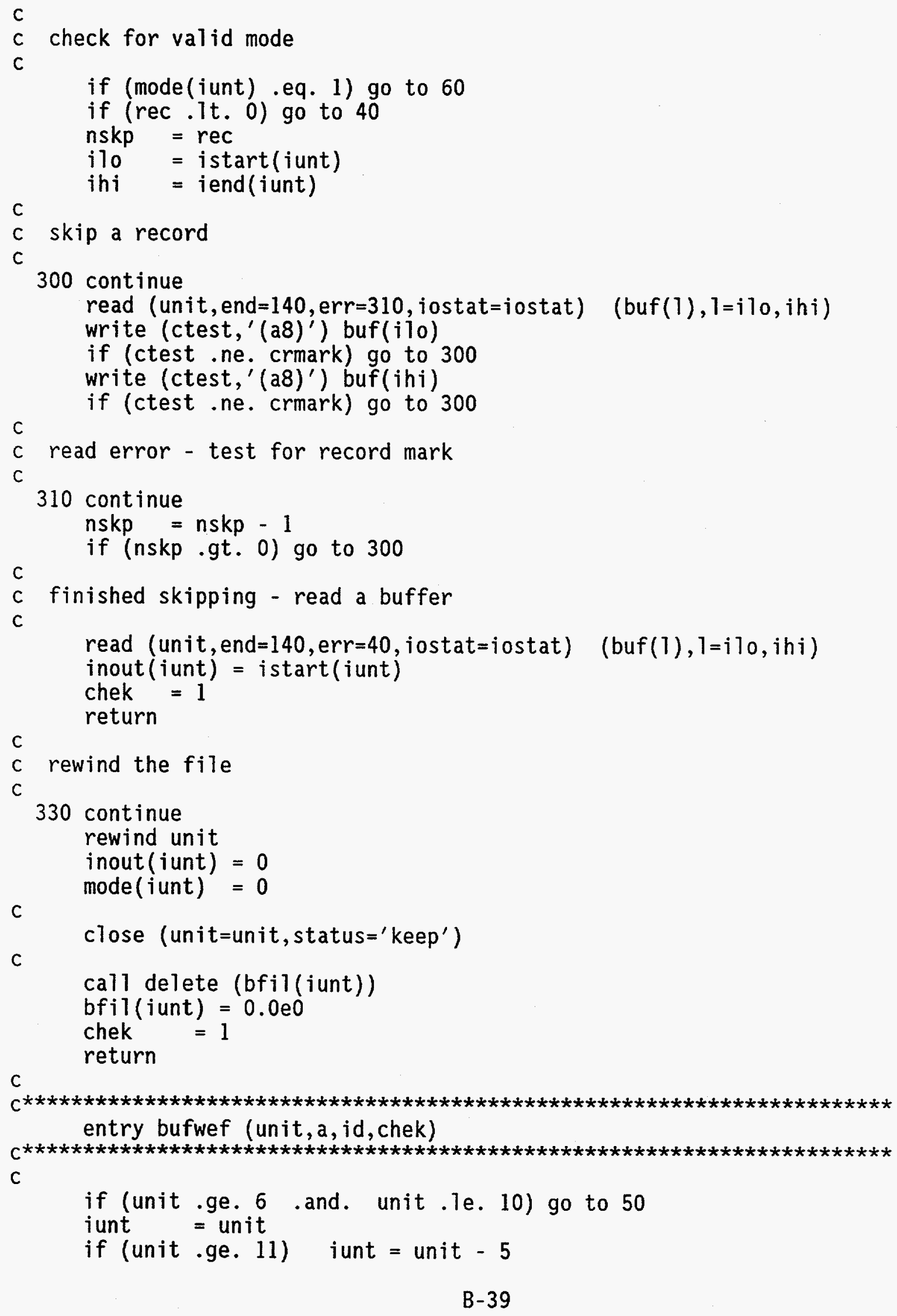


if (iunt.1e. 0 .or. iunt.gt. 10) go to 50

c

c check valid operation mode

c

i10 = istart (iunt)

ihi = iend (iunt)

if (mode(iunt) .eq. 2) go to 60

if (inout(iunt) .eq. istart(iunt)) go to 225

C

c pad buffer with record mark

C

$$
\min =\operatorname{inout}(\text { iunt) }
$$

C

$\operatorname{buf}(\min )=$ rmark

buf $($ ihi $)=$ rmark

C

write (unit,err $=40$, iostat=iostat) (buf(1), $1=i 10$, ihi)

c

c write a record mark

C

\section{5 cont inue}

buf $(i 10)=$ rmark

buf $($ ihi $)=$ rmark

c

write (unit,err $=40$, iostat=iostat) (buf $(1), 1=i 10$, ihi)

inout (iunt) $=$ istart (iunt)

C

if (id .ne. 15) go to 240

endfile unit

C

240 continue

chek $=3$

return

C

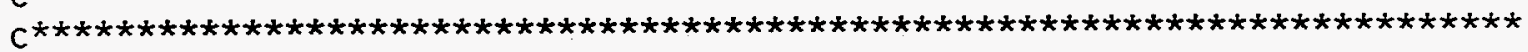

entry bufaev (unit, a, id, chek)

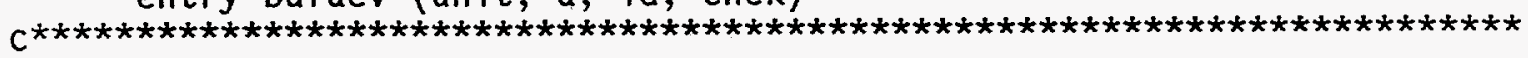

C

return

end

*deck hlfi

function hifinp $(n 1, n 2)$

c

c pack two integers into a real word (8-byte word for ibm)

C

c $n 1$ = integer to merge into top half of real word (input)

c n2 = integer to merge into bottom half of real word (input)

c

${ }^{*} \mathrm{cal} 1$ inpc

equivalence $(a 1, i a 1),(a 2, i a 2)$

C

ial $=n 1$ 


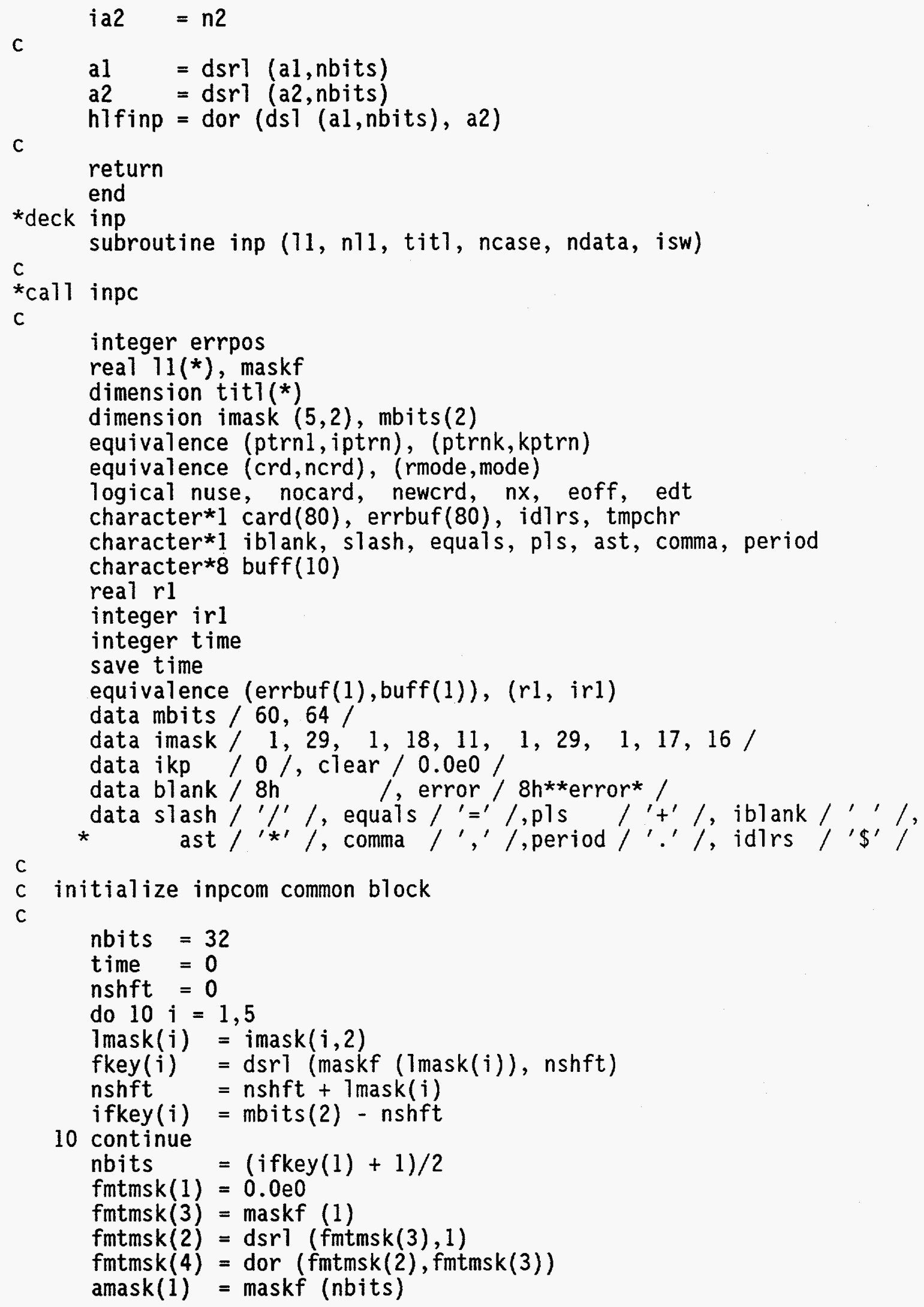




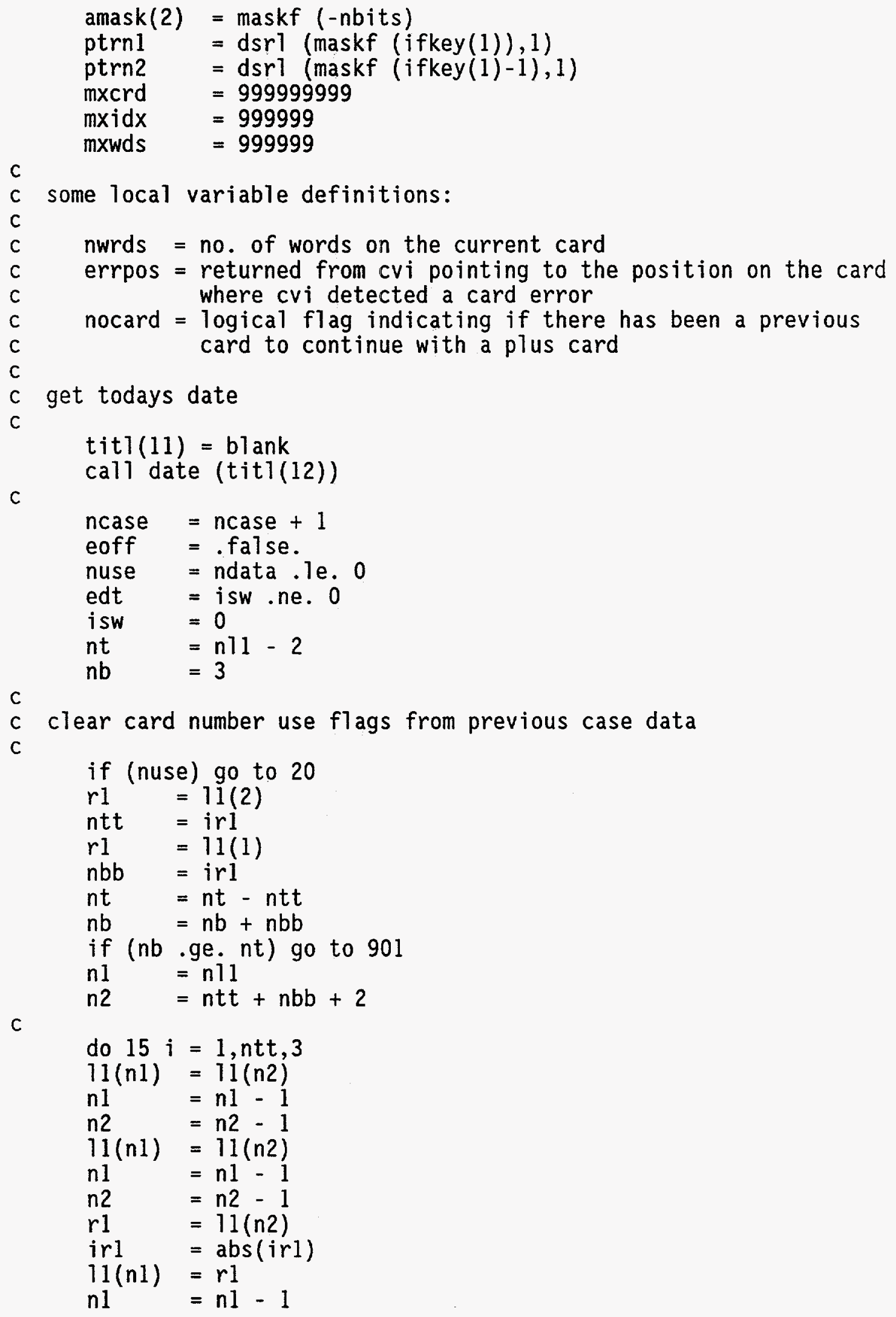




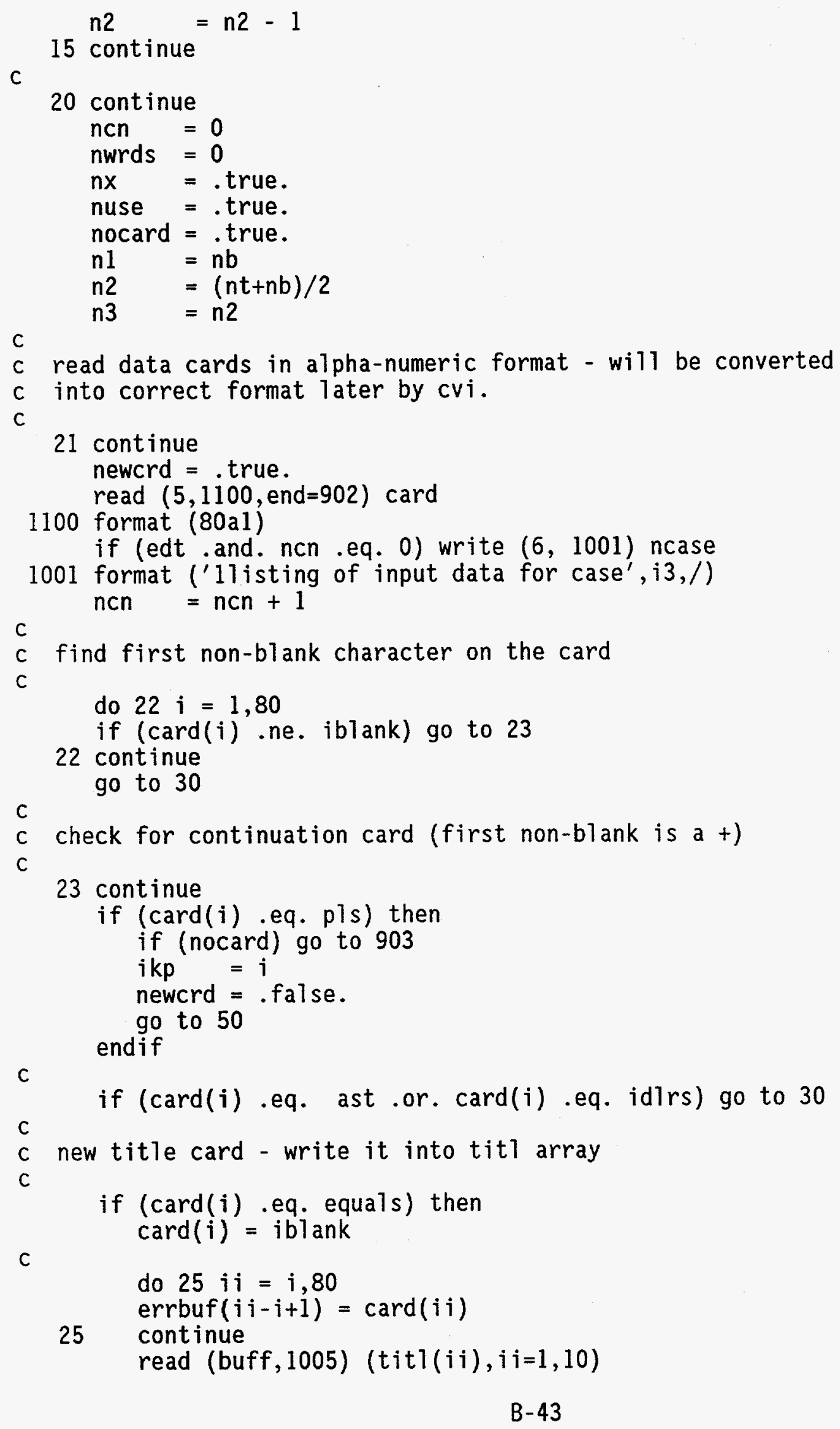




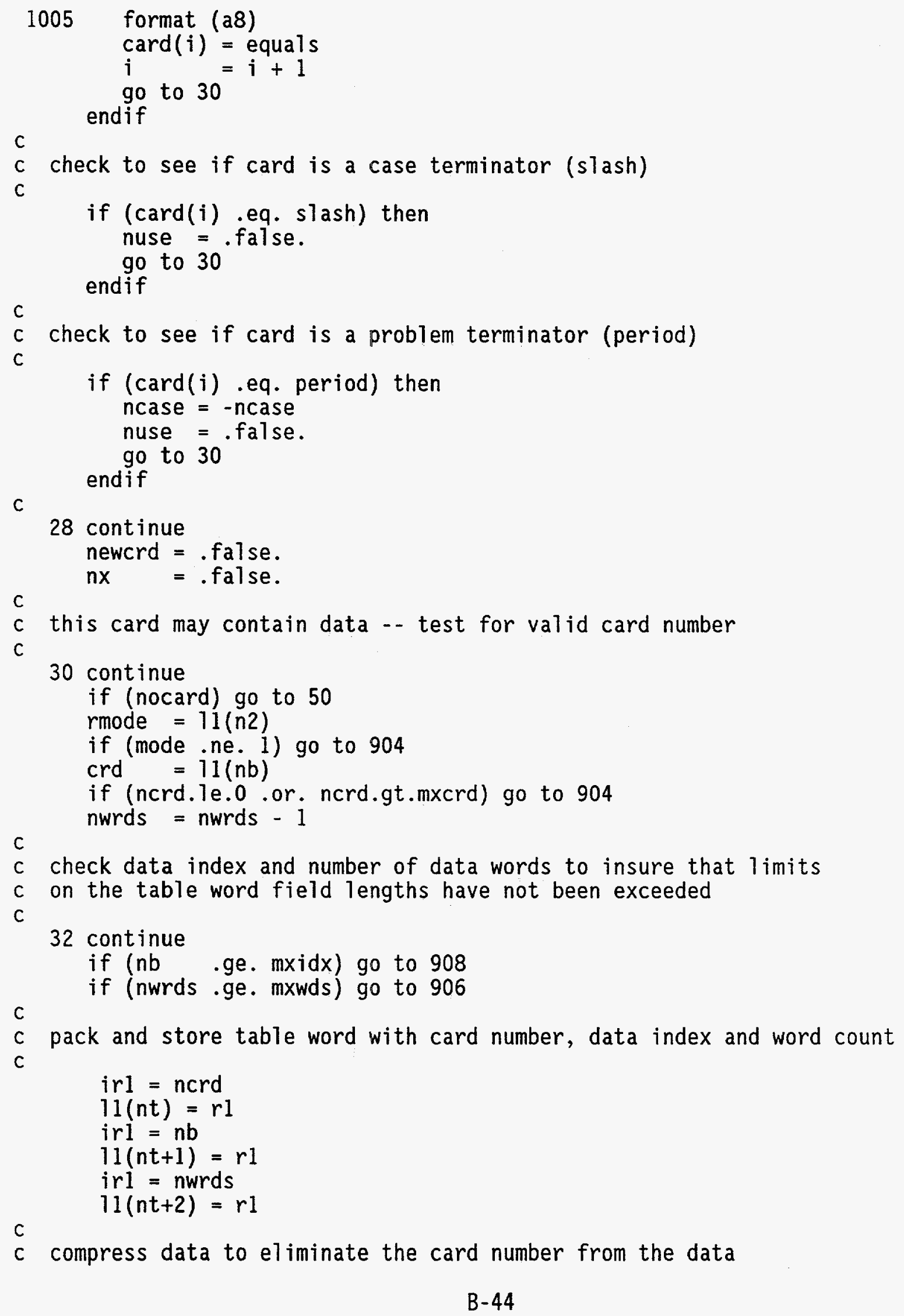

C

c compress data to eliminate the card number from the data 
c

if (nwrds .eq. 0) go to 45

do $35 i=1$, nwrds

$11(n b)=11(n b+1)$

$n b=n b+1$

35 continue

c

c pack data format flags into format mode word(s) and store after data

C

nmod $=$ nwrds

37 continue

nwpck = $\min 0$ (nmod, nbits)

cal1 inppck (11(nb), nwpck, $11(n 2+1))$

$\mathrm{nb}=\mathrm{nb}+1$

$n 2=n 2+$ nbits

$n 3=n 3$ - nbits

nmod = nmod - nbits

if (nmod.gt. 0) go to 37

c

c check if this is a replacement card

c

45 continue

if (ncrd .eq. 0) go to 49

if (nt eq. nl1-2) go to 46

c

$n 4=n t+3$

do $39 i=n 4, n 71,3$

$r 1=11(i)$

if (abs (irl) .eq. nerd) go to 38

39 continue

C

46 continue

if (nwrds .eq. 0) go to 40

49 continue

nt $=n t-3$

go to 40

C

c this is a replacement card

c

38 continue

if (edt) write $(6,3001)$

3001 format ( $1 \mathrm{~h}+, 90 \mathrm{x},{ }^{\prime} * * *$ this card is a replacement card. $\left.* * * \prime\right)$

$r 1=11(i+2)$

items $=i r 1$

$r 1=11(i+1)$

index $=$ irl

iwrds $=$ items $+($ items + nbits -1$) /$ nbits

$n 3=n b-1$

if (items .eq. nwrds) go to 42

do $100 \mathrm{j}=0,2$

100

$11(i+j)=11(n t+j)$

continue 


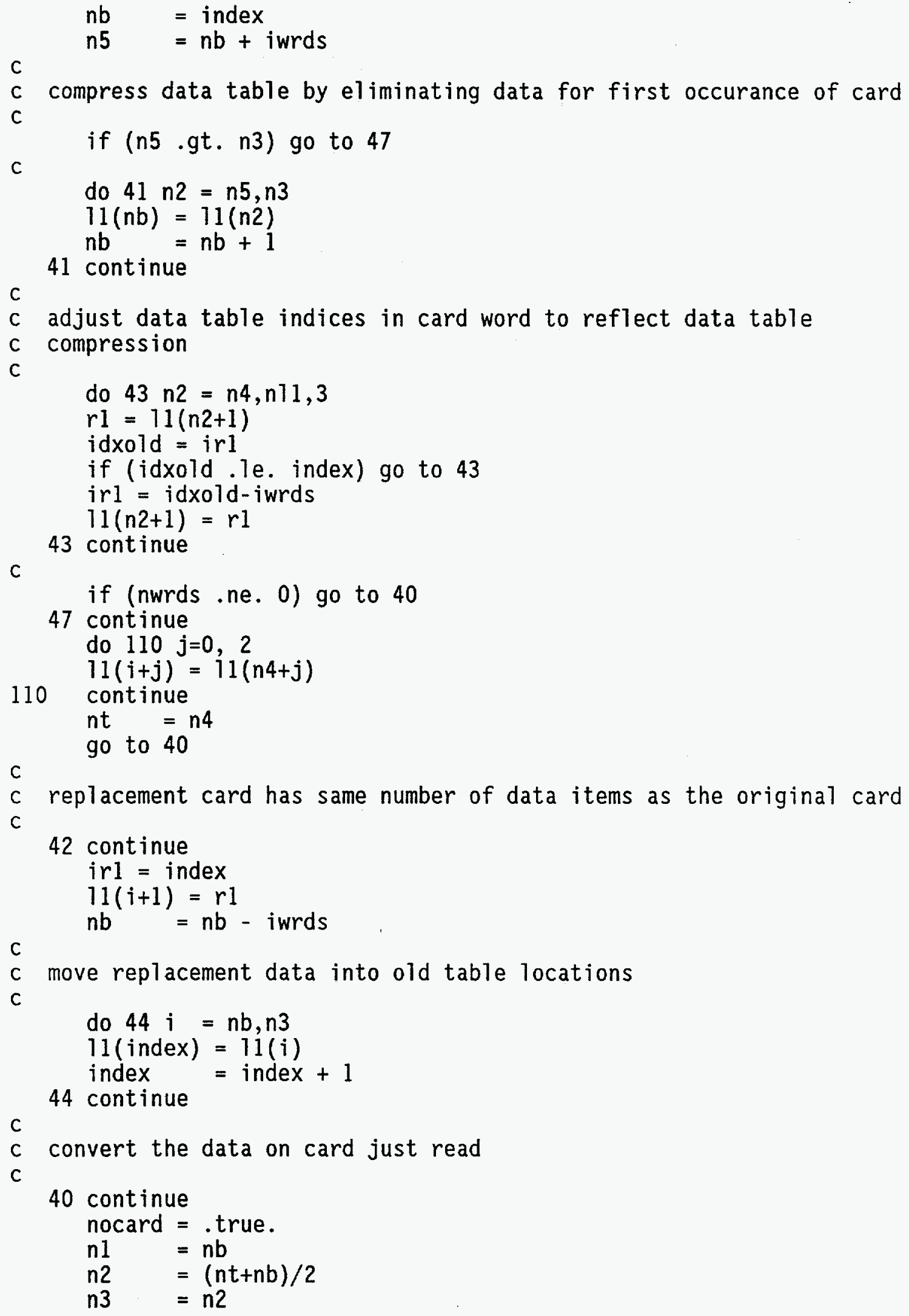




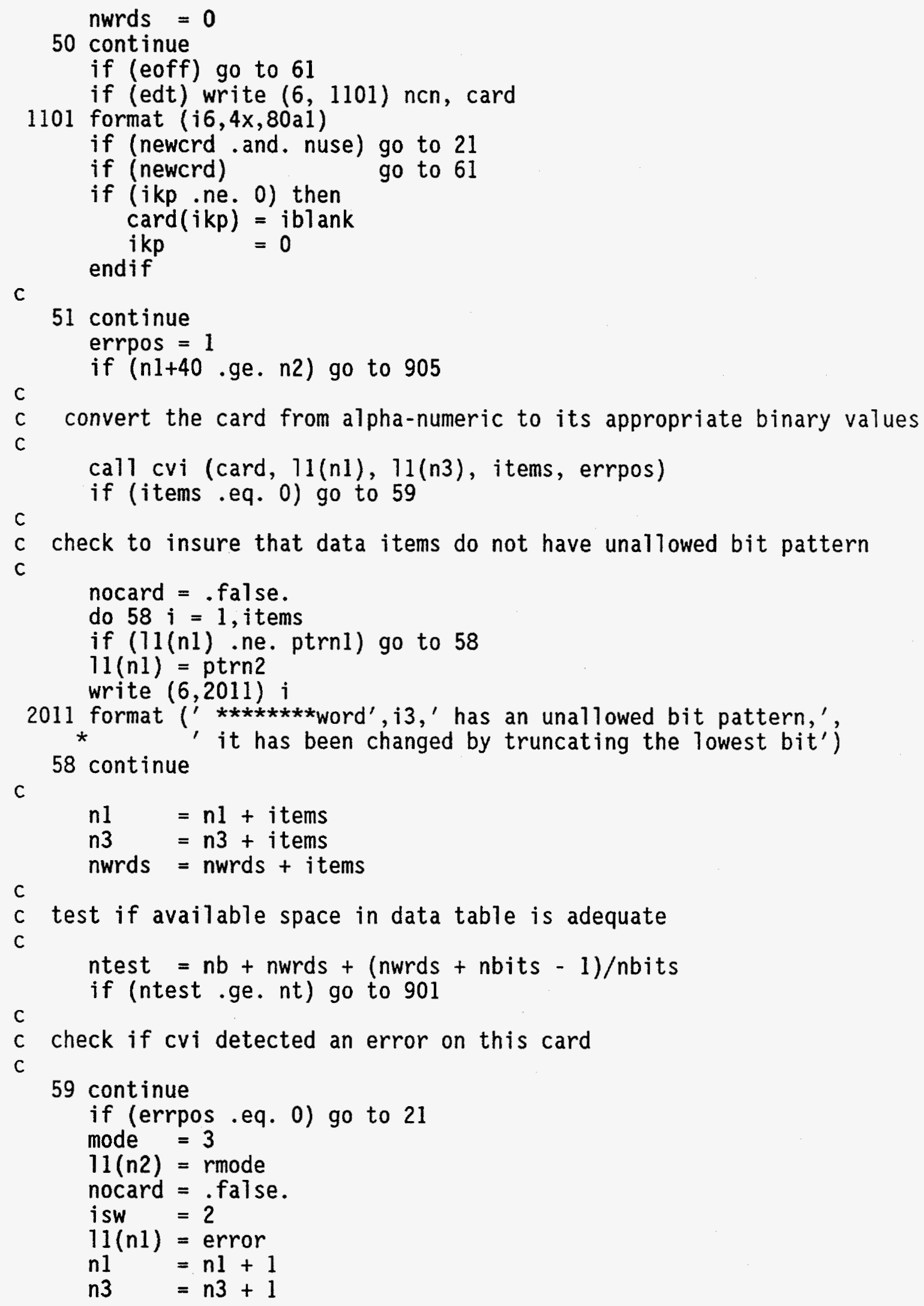

$c$

c check to insure that data items do not have unallowed bit pattern

c

nocard $=$. false.

do $58 i=1$, items

if $(11(n 1)$.ne. ptrn1) go to 58

$11(\mathrm{n} 1)=$ ptrn2

write $(6,2011) i$

2011 format $\left({ }^{\prime}, * * \star * \star * * * *\right.$ word',$i 3$, ' has an unallowed bit pattern,',

58 continue

c
$\mathrm{nl}=\mathrm{nl}+\mathrm{items}$
n3 $=n 3+$ items
nwrds $=$ nwrds + items

C

c test if available space in data table is adequate

c

ntest $=n b+n w r d s+($ nwrds + nbits -1$) /$ nbits

if (ntest.ge. nt) go to 901

c check if cvi detected an error on this card

c

59 continue

if (errpos .eq. 0) go to 21

mode $=3$

$11(\mathrm{n} 2)=$ rmode

nocard $=$.false.

isw $=2$

$11(n 1)=$ error

$n 1=n 1+1$

$n 3=n 3+1$ 
C

nwrds $=$ nwrds +1

do $52 i=1,80$

errbuf $(i)=$ iblank

c

52 continue

$\begin{array}{ll}\text { errpos } & =\min 0 \text { (errpos , 80) } \\ \text { errbuf }(\text { errpos }) & =\text { idirs } \\ \text { write }(6,1102) & \text { errbuf }\end{array}$

1102 format (10x,80al,4x,'\$ points to error on card above.')

$c$

c blank out the location where the error occurred, then

c go on to process the rest of the card by calling cvi again.

c

if (errpos.ge. 80 ) go to 21

do 53 i $=$ errpos, 80

tmpchr $=\operatorname{card}(i)$

$\operatorname{card}(i)=i b l a n k$

if (tmpchr. ne. iblank and. tmpchr. ne. comma) go to 53 go to 51

53 continue

go to 21

C

c finished reading cards -- finish bookkeeping and return

c

61 continue

$n t t=n 11-n t-2$

$n b b=n b-3$

ndata $=n t t+n b b+2$

irl $=\mathrm{nbb}$

$11(1)=r 1$

$\operatorname{irl}=n t t$

$11(2)=r 1$

if $(n x)$ ndata $=-$ ndata

c

c re-order card number table in descending order from top to bottom

c

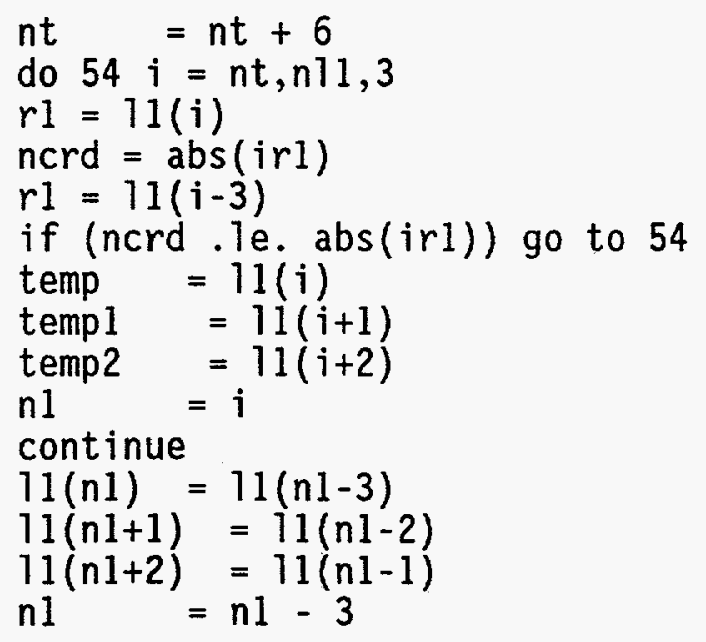




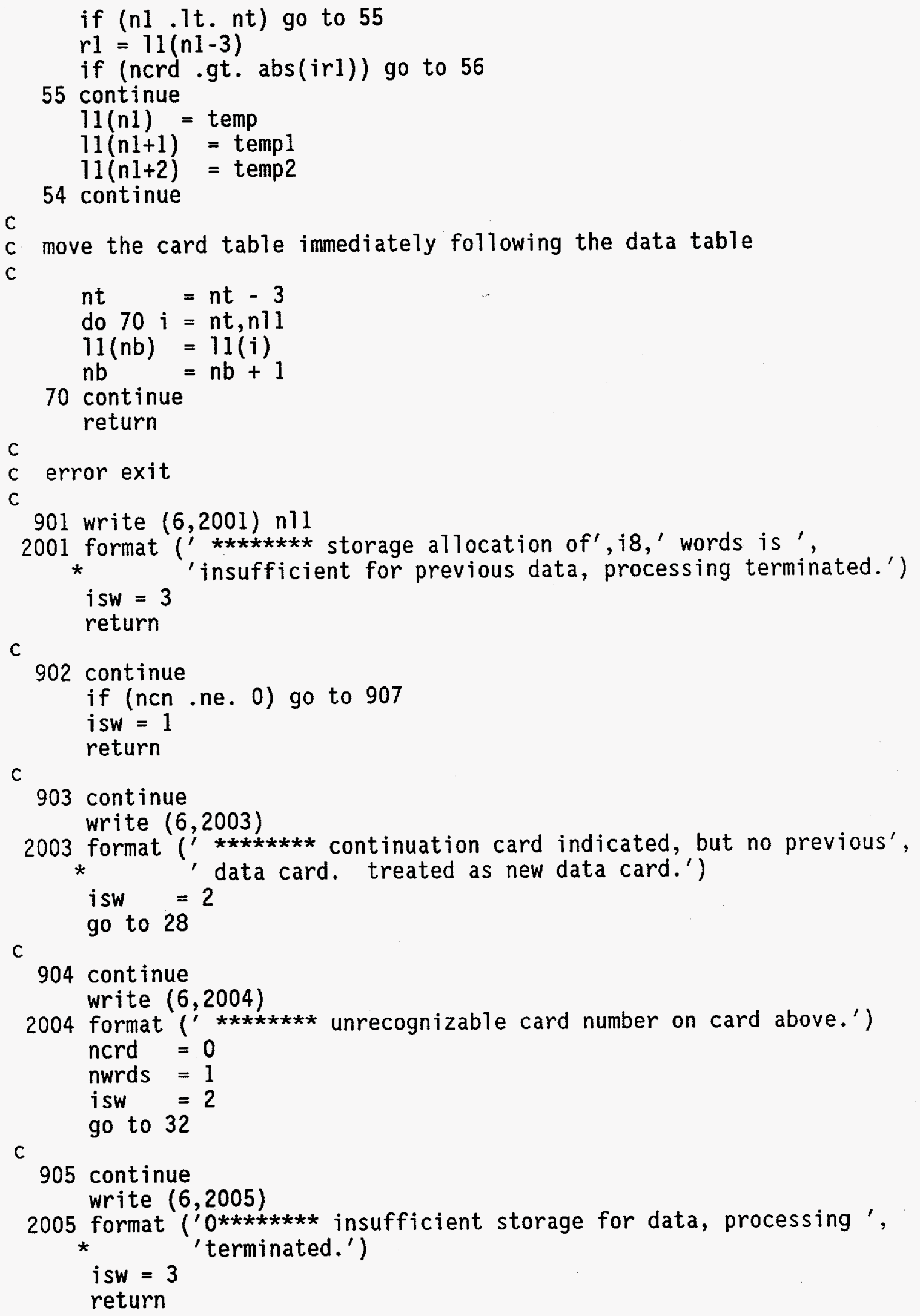


c

906 continue

write $(6,2006)$ mxwds

2006 format ('0******** number of words on card above exceeds the ', * $i s w=3$

return

c

907 continue

write $(6,2002)$

2002 format ('******** end of file encountered before end(.) card.') eoff $=$.true.

isw $=2$

ncase $=$-ncase

nuse $=$.false.

C

go to 30

908 continue

write $(6,2008) \mathrm{mxidx}$

2008 format (' $0 * * * \star * * * *$ number of data items on all cards processed',

* 'above exceeds the maximum of',i10,', execution terminated.') $i s w=3$

return

end

*deck conv

subroutine conv (a, xnum, type, 1start, lend, err)

C

C $\mathrm{a}=$ input array

c xnum = converted floating point or integer number

$c$ type = real variable containing integer format flag

c for xnum

$c=0$ integer or floating point zero

$\mathrm{c} \quad=1$ for integer

c $\quad=2$ floating point

c 1start = starting index in array a

$c$ lend $=$ ending index in array a

$c$ err $=$ error flag

C

character* $(*)$ a(1)

character*24 C

character* $1 \quad b(24)$

character*l decpt, blank, comma, ast, eee, dee, plus, minus

logical real, ldecpt, leee, err

integer num

equivalence $(c, b(1))$, (rnum, num)

C

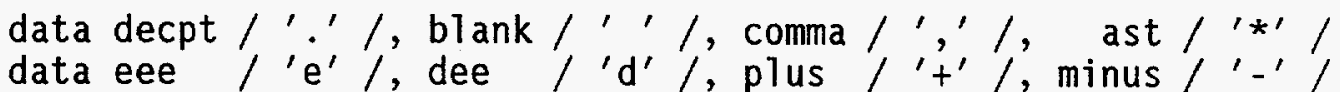

C

$c={ }^{\prime}$

err $=$.false.

ldecpt $=$.false. 


$$
\begin{aligned}
\text { real } & =\text { false } \\
\text { leee } & =\text { false } \\
\text { itype } & =0 \\
\text { lend } & =80
\end{aligned}
$$

$c$

c

check for integer of floating point format

do $10 i=1$ start, lend

if (a(i).eq.blank .or. a(i).eq.comma .or. a(i).eq.ast) go to 20

if $(a(i)$.eq. decpt) then

real $=$.true.

if (1decpt) then

err $=$.true.

lend $=i+1$

endif

return

endif

ldecpt $=$.true.

C

$\mathrm{c}$

$\mathrm{C}$

test for exponent with no preceeding decimal point

if ( $i . g t$. lstart) then

if (.not.1decpt) then

if $(a(i)$.eq.eee or. a(i).eq.dee or.

* a(i).eq.plus .or. a(i).eq.minus) then

leee $=$.true.

real $=$.true.

endif

$1 \mathrm{decpt}=$.true.

endif

10 continue

$i=81$

C

C convert to integer or floating point

C

20 continue

$$
\begin{aligned}
& \text { length }=\mathbf{i}-1 \text { start } \\
& \text { if (1ength } \cdot \text { gt. 24) go to } 70 \\
& \text { lend }=\mathbf{i}-1 \\
& \begin{array}{ll}
\mathrm{j} \quad=0 \\
\mathrm{k} \quad=0
\end{array}
\end{aligned}
$$

C

c move number into a character string -. then convert to a

c floating point number

C

if (.not.real) go to 40

if (leee) then

C

$c$ insert a leading decimal point before converting the number

c

$$
\text { length }=\text { length }+1
$$




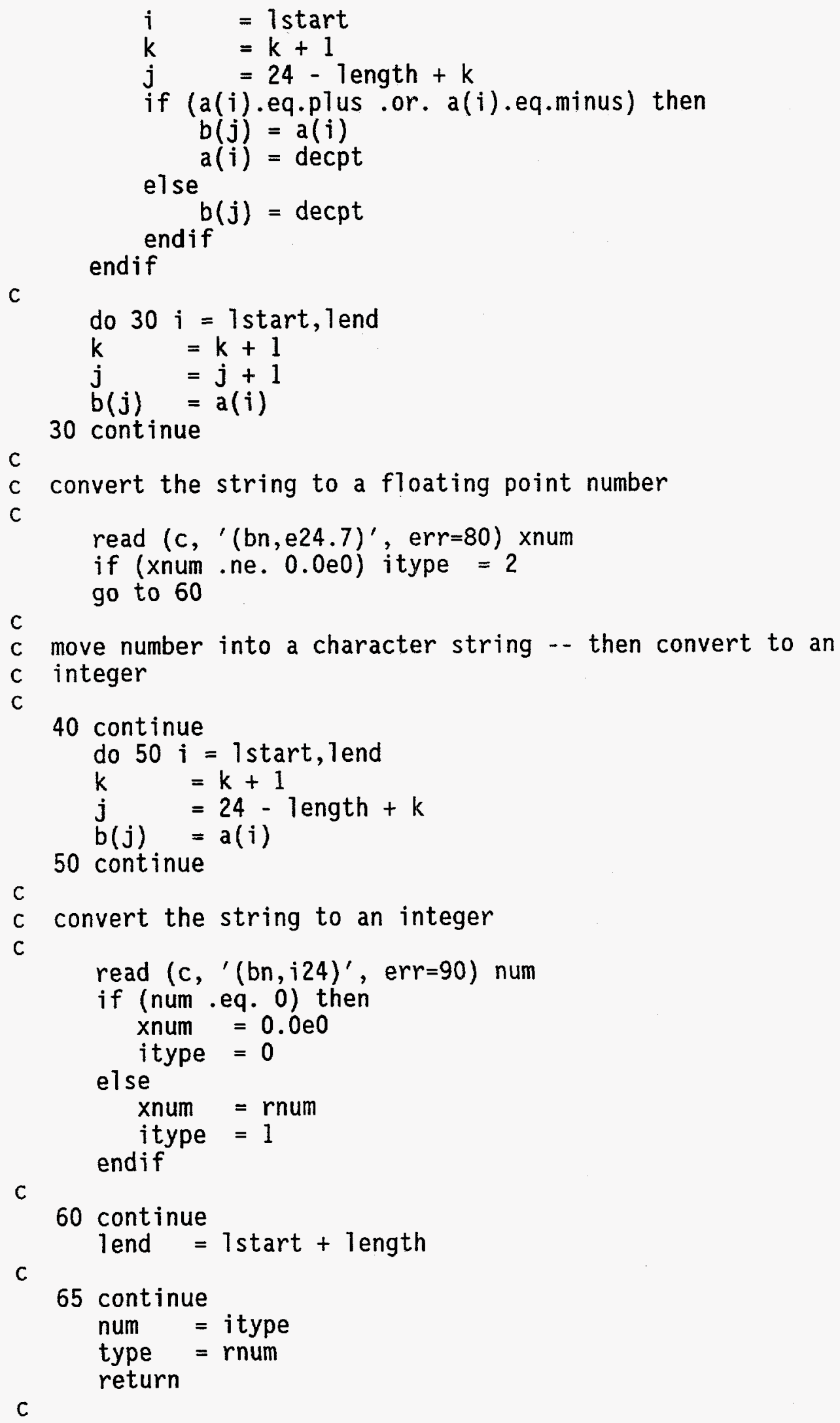


C

C

70 continue

write $(6,100) c$

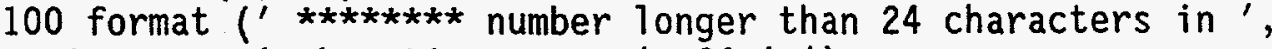

* err $=$.true.

itype $=0$

go to 65

80 continue

write $(6,110) \mathrm{c}$

110 format $(' * * * * * * * *$ floating point conversion error in ',

* 'subroutine conv ',a24,' '

err $=$.true.

go to 65

C

90 continue

write $(6,120)$

120 format $\left(l^{\prime}, * * * * * * *\right.$ integer conversion error in subroutine ', *

err $=$.true.

go to 65

end

*deck cvi

subroutine cvi (char, binary, cond, num, ipos)

C

character*1 char $\left.{ }^{*}\right)$

dimension binary $(*)$, cond $(*)$

logical err

character*1 blank, comma, astrsk, dollar, equal

character*1 d, e, slash, dp, apos, dblqt, number (13)

C

data number

$* / 0^{\prime},{ }^{\prime} 1^{\prime},{ }^{\prime} 2^{\prime}, 3^{\prime}, 4^{\prime},{ }^{\prime} 5^{\prime},{ }^{\prime} 6{ }^{\prime}$

data dblqt', "l';

C

c find first non-blank character.

C

ipos $=0$

num $=0$

do $1011=1,80$

if (char(11). ne. blank) go to 20

10 continue

return

C

c process data card by first determining whether it is numeric or hollerith

C

20 continue

1start $=11$ 


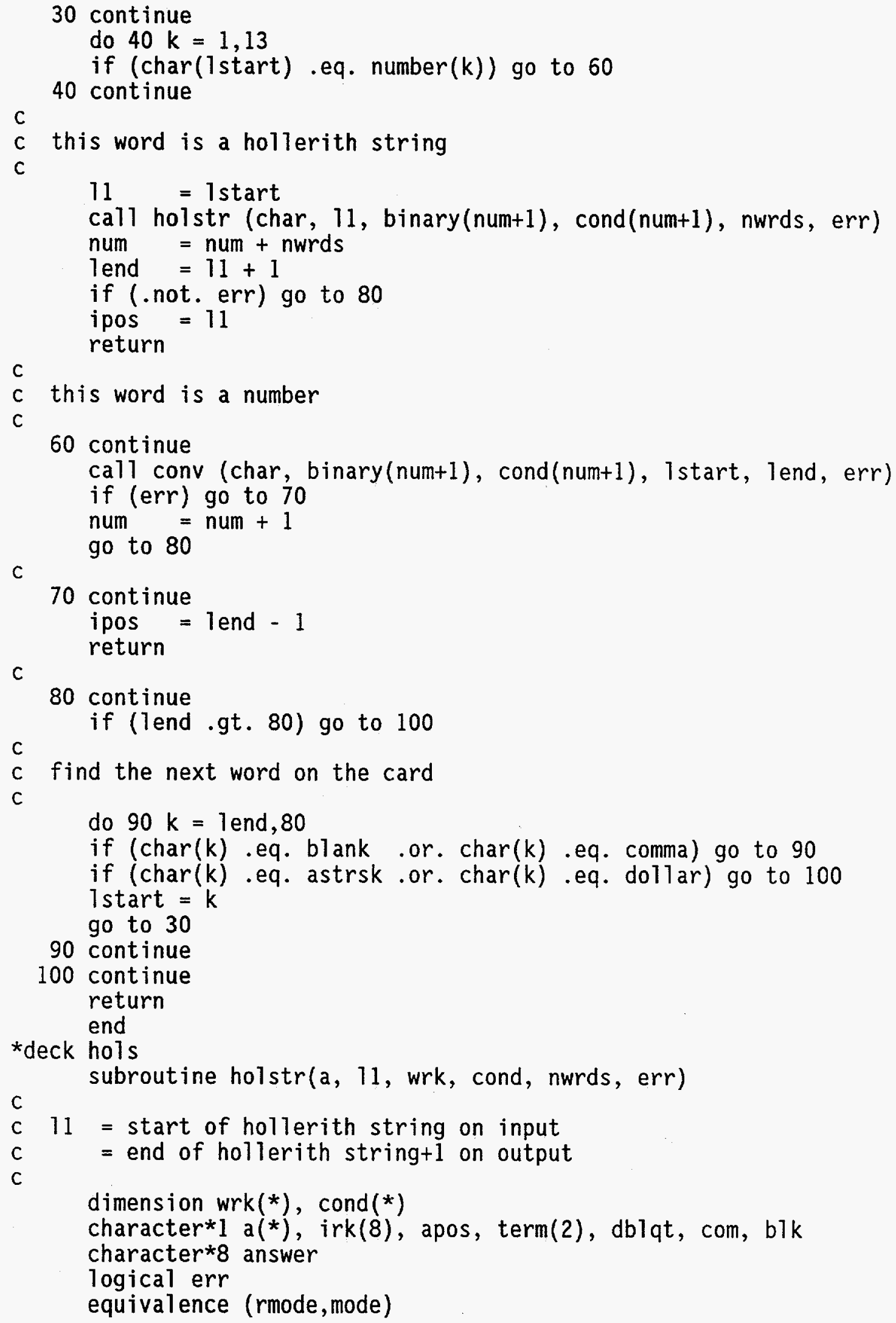




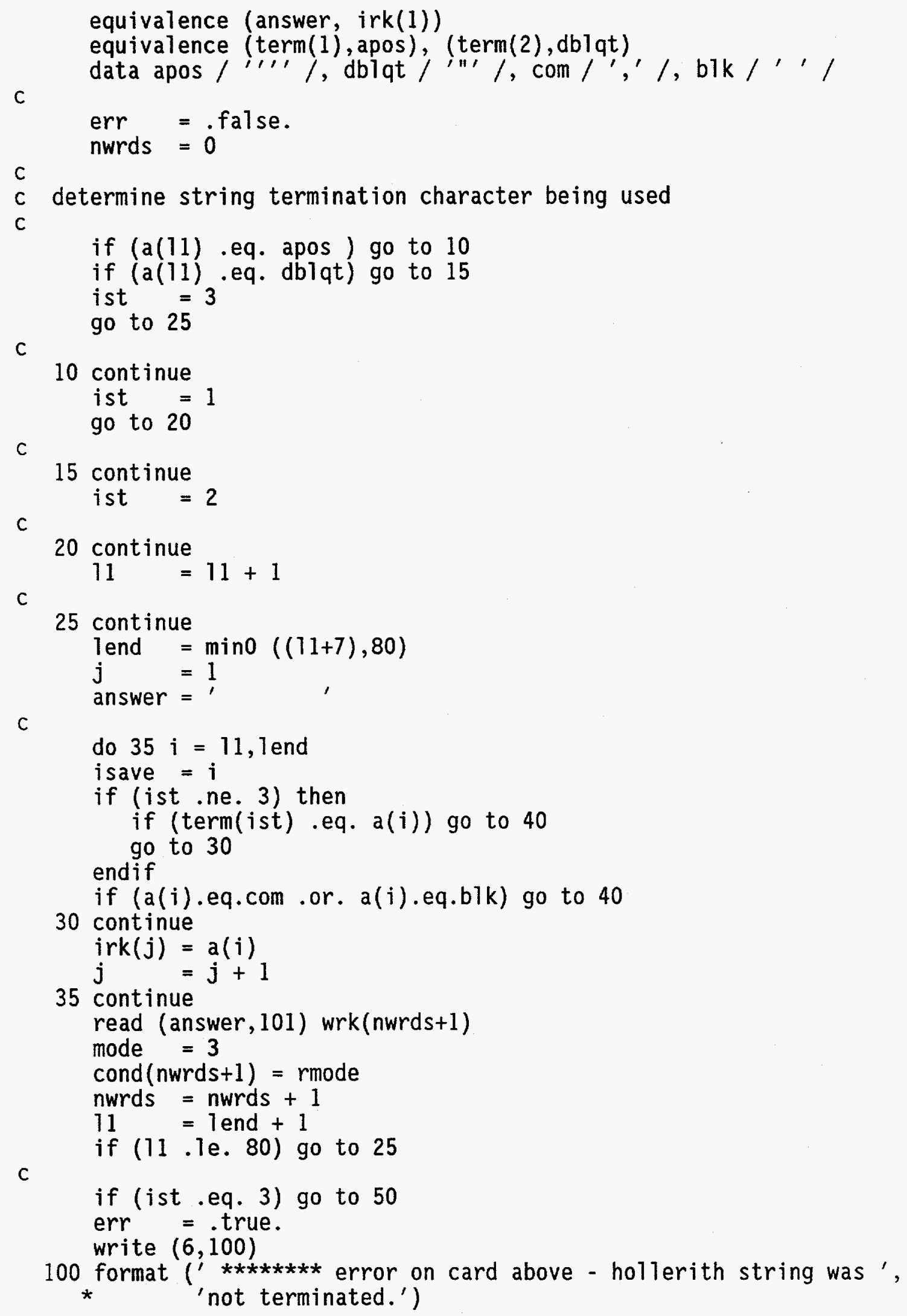




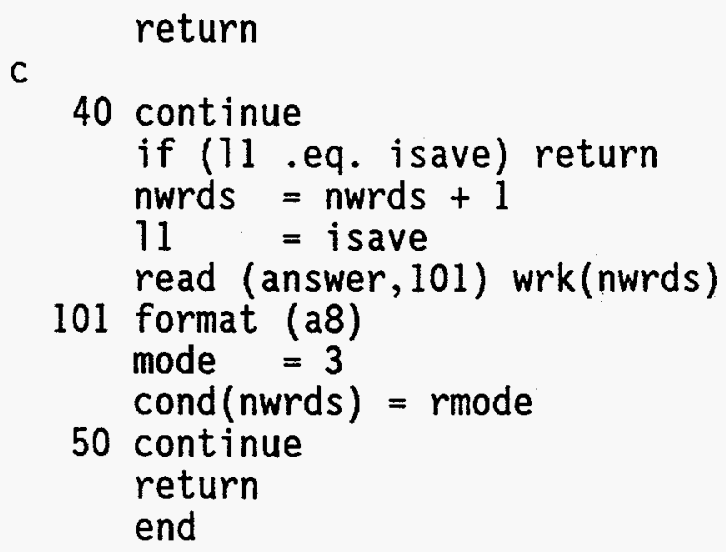




\section{APPENDIX C}

\section{RETRAN-02 UPDATE DECK}

The correction decks to build the CRAY version of RETRAN-02 from the CDC source file are in three update decks labeled with the identifiers 'ident lan101', 'ident 1an102', and 'ident 1an103'. These correction decks are not independent and must be processed sequentially to obtain a correct source. The RETRAN-02 source is written in lower case characters.

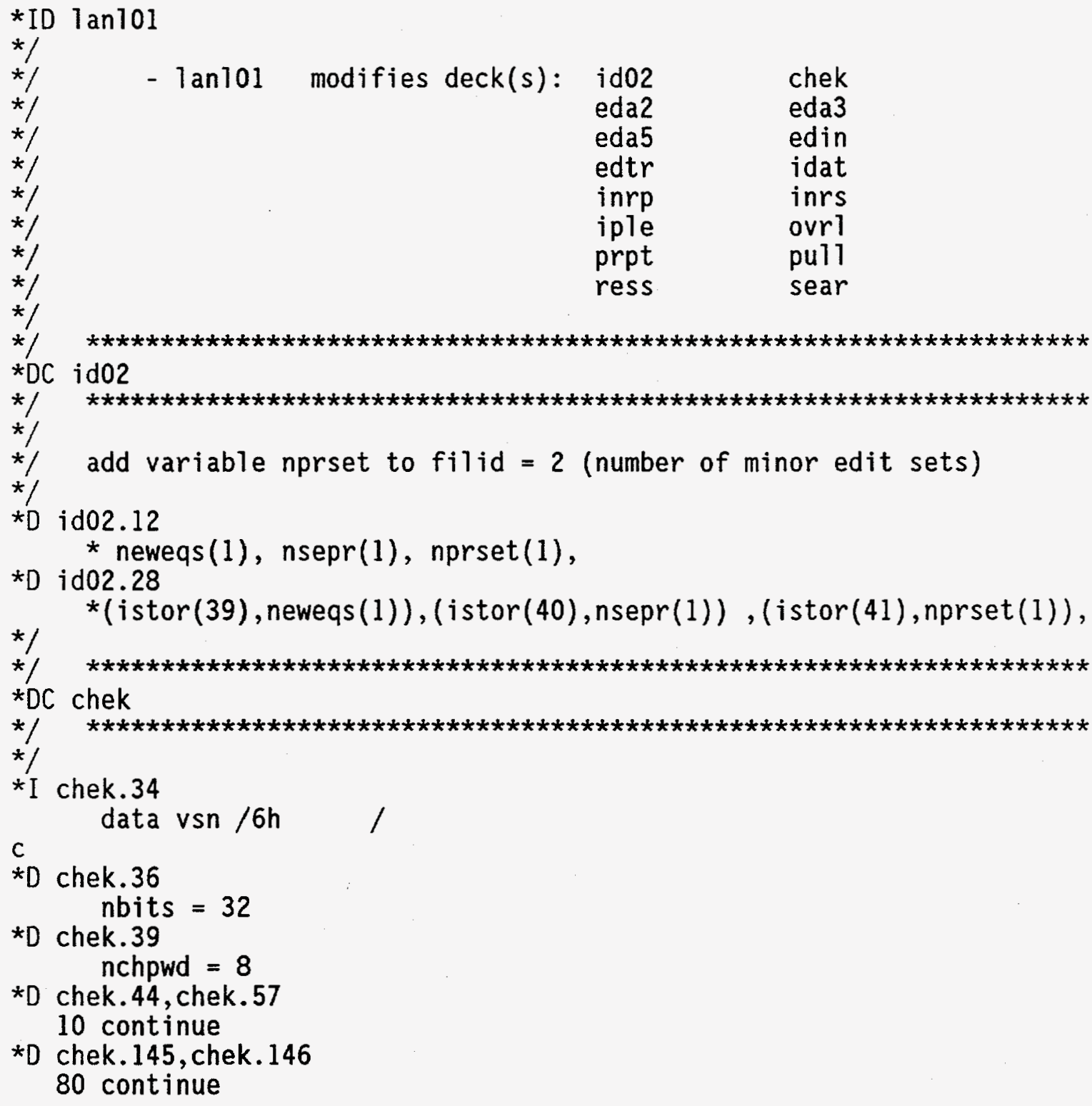


*D chek. 164

write $(6,700)$ (rstor $(i d x s c r+i-1), i=1,1$ en)

700 format $(1 x, 6 z 20)$

*/

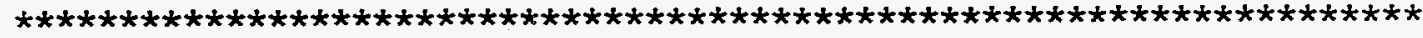

$\star D C$ edal

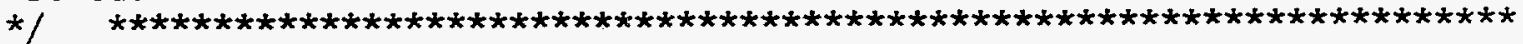

$* /$

*D edal.139

nbits $=32$

*D edal.141

nbyte $=8$

*D edal.146

Ten $=-3$

*/

*/

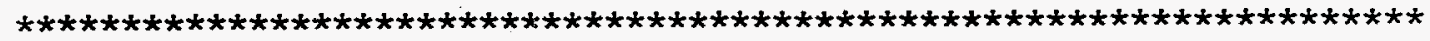

$*$ DC eda2

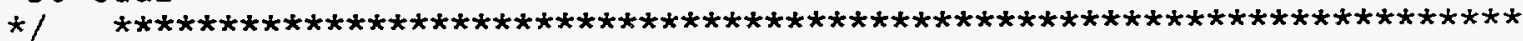

*

$* D$ eda2.181

nbits $=32$

*D eda2.183

nbyte $=8$

*D eda2.188

len $=-3$

*/

$\star 1$

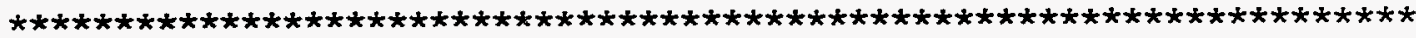

$\star D C$ eda3

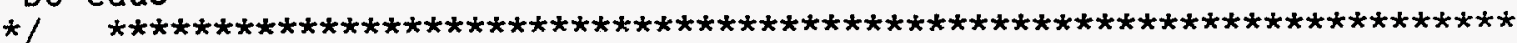

*

*D eda3.200

nbits $=32$

${ }^{*}$ D eda3.202

nbyte $=8$

${ }^{*} \mathrm{D}$ eda3.207

1en $=-3$

$* /$

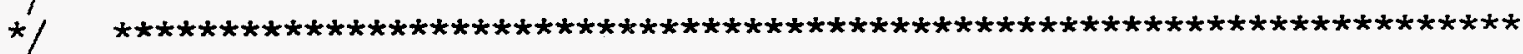

$\star D C$ eda 4

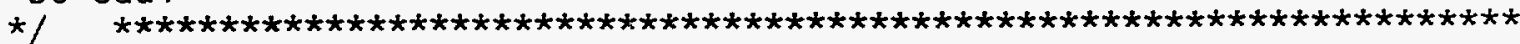

*/

$* \mathrm{D}$ eda4.167

nbits $=32$

*D eda4.169

nbyte $=8$

*D eda 4.174

Ten $=-3$

$\star /$

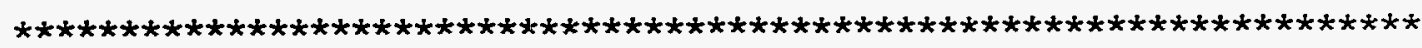

${ }^{*} D C$ eda5

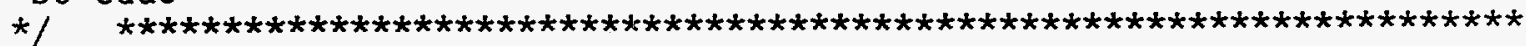
$* /$ 
*D eda5.201

nbits $=32$

*D eda5.203

nbyte $=8$

*D eda5.208

len $=-3$

$* /$

$* D C$ edin

*I

*1

${ }^{*}$ D edin. 68 , edin. 69

nbits $=32$

nbyte $=8$

*D edin. 280 , edin.283

*1

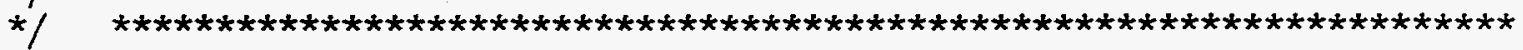

$*$ DC edit

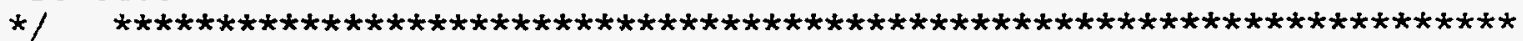

*/

*D edit. 267 , edit. 281

315 continue

*D edit. 304 , edit. 306

cal1 bufout $(3$, rstor (idxb), nedv, iok, last, dumy)

if (iok.ne.1 .or. nedv.ne. last) then

write $(6,801)$

801 format (' $0 * * * * * * * *$ bufout error writing printer plot records') nogo $=$.true.

call fail

end if

$* 1$

$* D C$ edtr

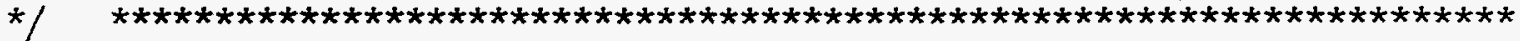

*/

$* D$ edtr. 23

$\star D$ edtr. 40

nbits $=32$

*D edtr. 47 , edtr.50

$*$ D edtr.79, edtr.80

${ }^{*} \mathrm{D}$ edtr.85, edtr.95

35 write $(6,700)$ ivol

700 format ('O $0 * * * * * * *$ end of volume processing disabled - fail calle $\left.\star d^{\prime}\right)$

call fail

go to 200

*D edtr. 134 , edtr .137

*D edtr. 142

write $(6,620)$ iplot

*D edtr.147

write $(6,630)$ kunit

$*$ D edtr. 160 
write $(6,660)$

*D edtr.167, edtr.174

620 format ("0 $0 * * * * * * *$ data record", i6, " can not be reached")

630 format (" $0 * * * * * * * *$ bufskp error on unit", $i 3$, " in subroutine editre *")

*D edtr. 177 , edtr. 178

*/

660 format ("0 tape is not a relap/e data tape"/" execution deleted")

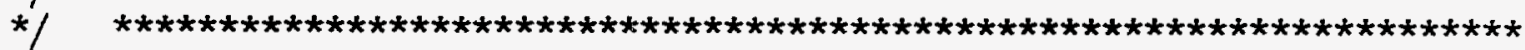

$* D C$ idat

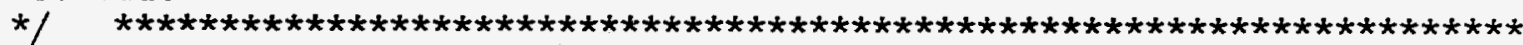

*1

*D idat.55, idat. 80

*1

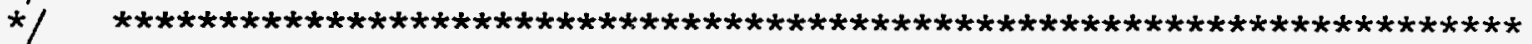

$* D C$ iede

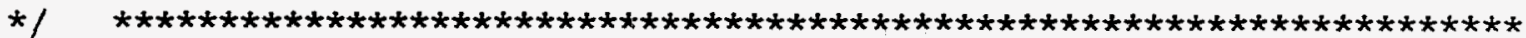

*/

$*$ D iede. 29

nbits $=32$

*D iede. 87, iede. 128

$$
\text { num }=1
$$

${ }^{*} \mathrm{D}$ iede. 130 , iede.133

*/

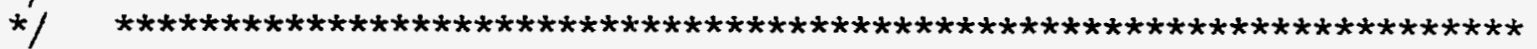

*DC inrp

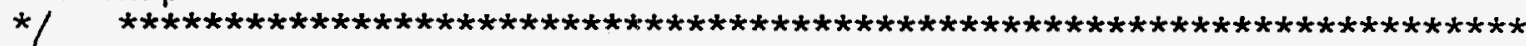

*1

*D inrp.34

nbits $=32$

*D inrp.146, inrp. 198

num $=1$

*D inrp.200, inrp.208

*/

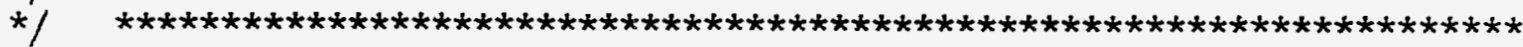

$*$ DC inrs

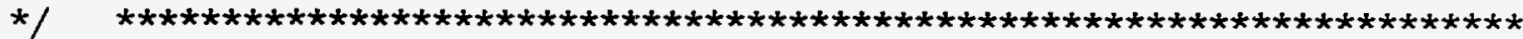

*1

*D inrs. 36

nbits $=32$

*D inrs.78, inrs. 125

num $=1$

*D inrs.140,inrs.199

${ }^{*}$ D inrs.232, inrs.240

write $(6,720)$

*D inrs.244, inrs.264

*D inrs.272, inrs.273

*D inrs.316

720 format ("0 restart from a retran data tape ")

*1

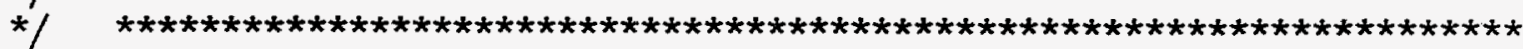

$* D C$ intv 
$\star D$ intv. 33

nbits $=32$

${ }^{\circ} \mathrm{D}$ intv. 150 , intv .170

*D intv. 172 , intv. 200

*/

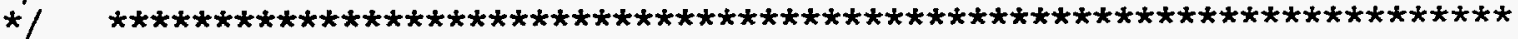

$\star D C$ iple

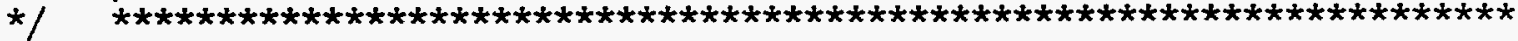

*/

*D iple.135

nbits $=32$

*D iple.157,iple.195

*D iple.197

$* 1$

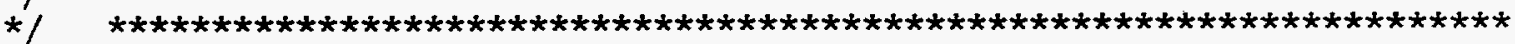

*DC ovrl

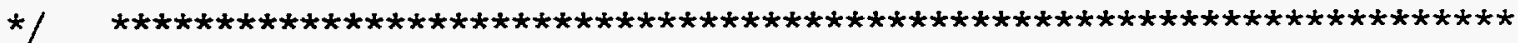

*

*D ovr 1.18

nbits $=32$

*D ovr1.109, ovr1.126

${ }^{*}$ D ovr1.134

$* 1$

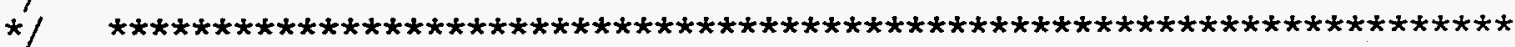

*DC plta

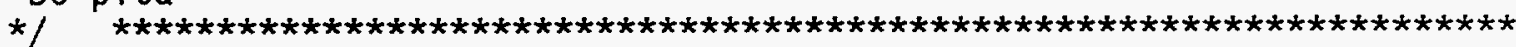

*1

*D plta.41

nbits $=32$

*D plta.59,plta.97

*D plta.106

*/

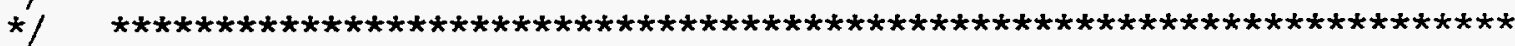

$* D C$ prpt

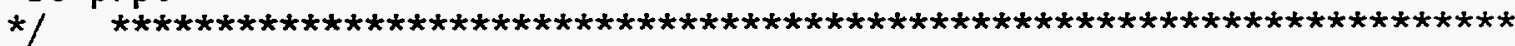

*1

*I prpt.12

real nextid

$C$

*D prpt.15,prpt.17

ndata $=\operatorname{nprset}(\mathrm{i2})$

*I prpt.41

prplot $=$ nextid $(0)$

lenplt $=$ nplts

call reserv (prplot, lenplt, 2 , index)

*D prpt. 43

*D prpt. 52

call bufin (3, rstor(index), lenplt, iok, last, dum)

if (iok.ne. 0 .or. last.ne.lenplt) then write $(6,1001)$ i 
1001 format (' $0 * * * * * * * *$ bufin error reading printer plot data file *for minor edit no. ', i5) end if

${ }^{*}$ D prpt. 58 call fail

ca 11 bufskp(3, 1, -1, iok)

if (iok.ne.0) then write $(6,1002)$

1001 format (' $0 * * \star \star \star * * *$ bufskp error reading printer plot data file * for minor edits') end if

*D prpt. 62 call fail

50 continue

*/

$*$ DC pul1

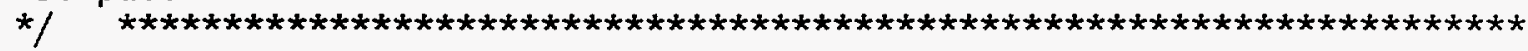

$* 1$

$\star D$ pul1.25

nbits $=-32$

*/

*DC rdtp

*D rdtp. 33, rdtp. 34

nbyte $=8$

nbits $=32$

*D rdtp. 37

$$
\text { nchars }=32
$$

*D rdtp.91

*D rdtp.103,rdtp. 104

*D rdtp. 147

*D rdtp.204,rdtp.207

call reserv (datid, isiz3, 2, index)

*I rdtp.208

c write all data from dataset i to disk file

call bufout ( 3 , rstor(index), isiz3, iok, next, dum)

if (iok.ne.0 .or. Tast.ne.isiz3) then irec $=1$

write $(6,1001)$ irec, $i$

1001 format (' $0 * * * * * * * *$ bufout error writing plot data file for rec *ord ', i4,' in data set ', i4) call fail

end if

*D rdtp. 227, rdtp. 248 write $(6,1002)$

1002 format (' $0 * * * * * * * *$ error in reading plot data file. Multiple tap *es requested, feature has been disabled')

call fail

*D rdtp. 257

go to 600

295 continue

*I rdtp. 260 
call bufout (3, rstor(index), isiz3, iok, next, dum)

if (iok.ne.0 or. last.ne.isiz3) then

irec $=$ iplot

write $(6,1003)$ irec, i

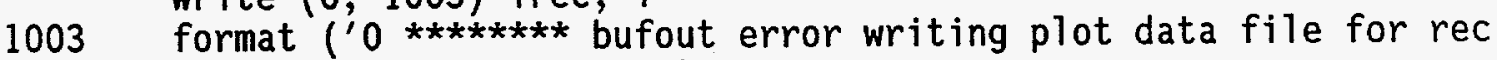
*ord ', i4,' in data set ', i4)

call fail

end if

*D rdtp. 269, rdtp .272

310 call delete(datid)

npsets (idxdat) = iplot

*D rdtp.333

470 continue

*D rdtp.339

480 continue

*D rdtp.341

530 write $(6,830)$ kunit

*D rdtp.348

write $(6,820)$

${ }^{*}$ D rdtp. 351, rdtp. 352

*D rdtp.354,rdtp.357

820 format ("0 $0 * \star \star \star * *$ tape is not a retran data tape"/

* " execution deleted")

830 format ("0******** bufskp error on unit", $i 3$, " in subroutine redtap *")

$* 1$

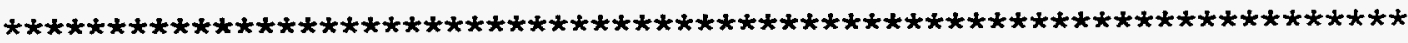

$* D C$ ress

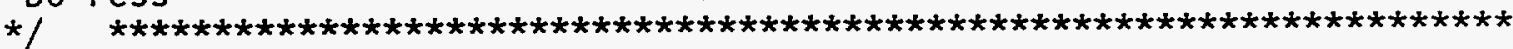

*/

*I ress. 25

nosemi $=$.true.

*/

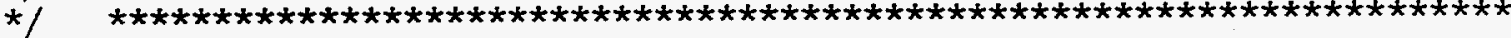

$* D C$ sear

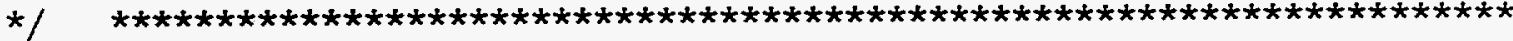

*/

*D sear. 42 , sear. 43

nbits $=32$

nbyte $=8$

$\star /$

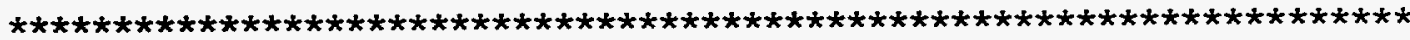

$\star D C$ tpbc

*I

$* 1$

$*$ D tpbc. 50

nbits $=32$

*D tpbc. 78, tpbc.79

*D tpbc. 248, tpbc. 269

write $(6,1002)$

1002 format ('O $0 * * * * * * * *$ error in reading time-dependent volume data fi *le. Multiple tapes requested, feature has been disabled') 


\section{call fail}

go to 251

*D tpbc. 320, tpbc. 322

315 format ("0******** error, data record", i6," cannot be found")

${ }^{*} \mathrm{D}$ tpbc. $331, \mathrm{tpbc} .342$

340 continue

*/

$*$

$* 1$

$\star 1$

$*$

$* 1$

*ident 1 an 102

- End of 1 an101

*)

$*$

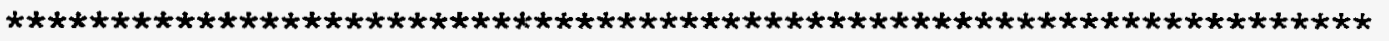

$* 1$

* corrections to $r 2 \bmod 4$

$*$

*/

$* /$

$* /$

$* /$

*/

$*$

$* /$

$*$

*/

${ }^{*} \mathrm{C}$ rman

$* d \quad r m a n .2,3$

program rmain

* $j$ rman.3510

open (unit $=5$, iostat $=$ ierr, err $=501$, file $={ }^{\prime}$ tape $5^{\prime}$, status $={ }^{\prime} 01 d^{\prime}$,

* access $=$ ' sequential', form='formatted')

open (unit=6, iostat $=$ ierr, err $=502$, fi $1 e={ }^{\prime}$ tape6', status $={ }^{\prime}$ new',

C

* access $=$ ' sequential' , form='formatted')

*d $\operatorname{rman} .3516$

$$
i=10 c x(r \operatorname{stor}(2))-10 c x(r \operatorname{rtor}(1))
$$

*d rman. 3553

*d rman.3558

$*_{i}$ rman.3561

C

501 write $(6,9001)$ ierr

9001 format ('0 $0 * \star \star \star \star \star * *$ error number ', i5,' returned attempting to ope $*_{n}$ tape $5^{\prime}$ )

c go to 110

502 write $(6,9002)$ ierr

9002 format ('O $0 * * * * * * *$ error number ', $i 5$, ' returned attempting to ope *n tape6')

*1

go to 110 


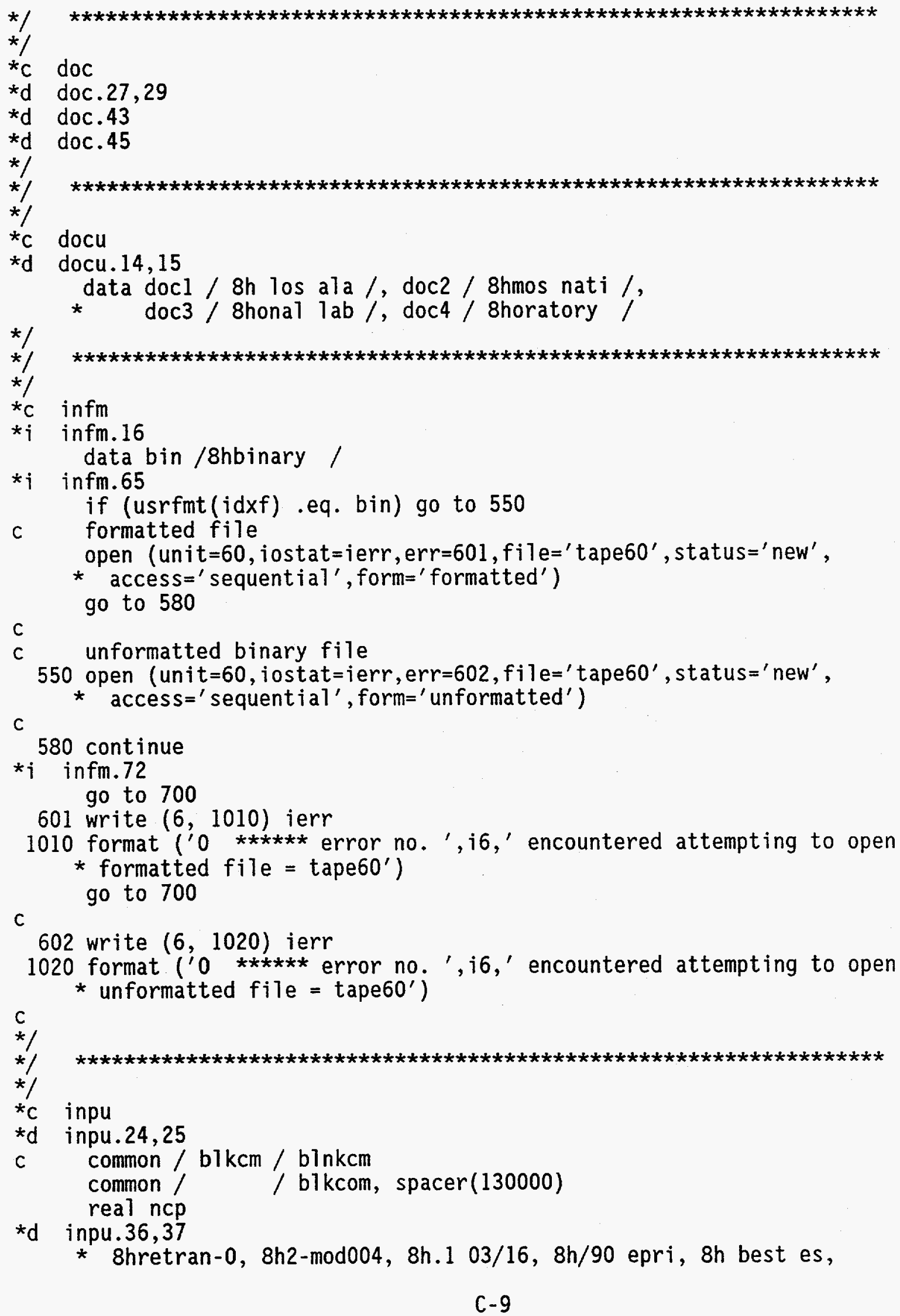


$*_{d}$ inpu. 51

* 8htimate f, 8hluid hyd, 8hraulics, 8hanalysis, 8h program,

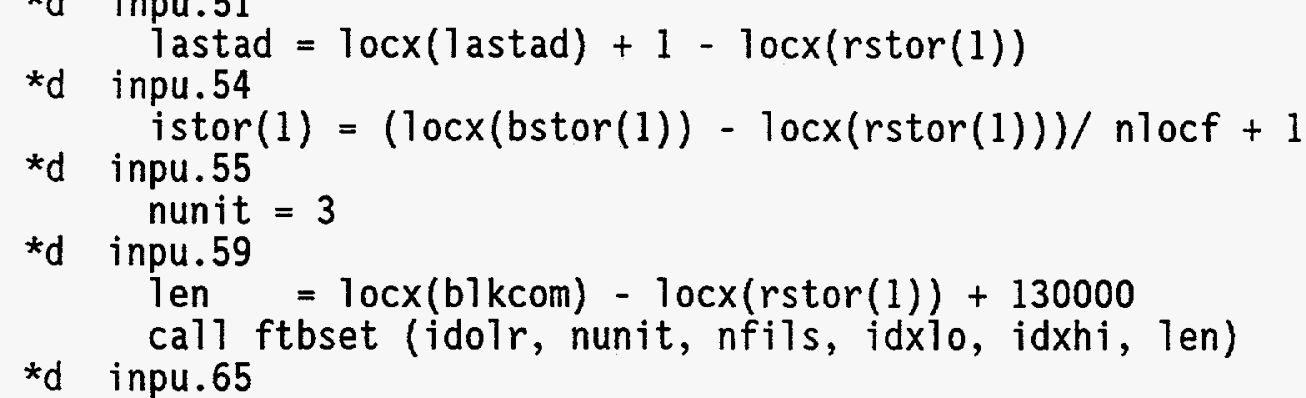

$*$ d inpu. 65

$\mathrm{nCp}=1000.0$

$* d$ inpu. 69,71

if (ibmrun) 1en $=10 \mathrm{cx}(\mathrm{b} 7 \mathrm{nkcm})$

if (.not.ibmrun) $1 \mathrm{en}=1 \mathrm{ocx}(\mathrm{b} 1 \mathrm{kcom})$

len $=($ len -1 ocx $(1$ astad $)+$ nlocf -1$) /$ nlocf

${ }^{2} \mathrm{~d}$ inpu.211

Ten $=10 c x(b 1 \mathrm{kcom})-10 \mathrm{cx}($ rstor $(1))+130000$

call ftbset (idolr, nunit, nfils, idxlo, idxhi, len)

$\begin{array}{ll}* / & \\ * & * * * \\ * / & \\ * c & i t r n\end{array}$

$*$ d itrn.25

nbits $=32$

*/

*/

${ }^{*} \mathrm{c}$ posi

${ }^{*}$ d posi.76,77

write $(6,650)$ (rstor (idxscr+k-1), $k=1$, len)

*/

650 format $(1 x, 6 z 20)$

*/

$*$

$* /$

${ }^{*} \mathrm{c}$ plta

$*_{r}$ plta.76

$* /$

*/

$*$

${ }^{*} \mathrm{C}$ prpt

*d 1 an101.77

1002 format (' $0 * * * * * * * *$ bufskp error reading printer plot data file

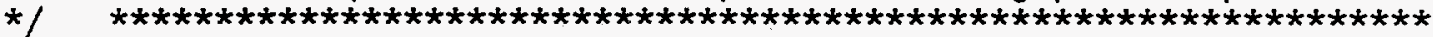

$*$

${ }^{*} \mathrm{c}$ reso

*d reso.51,322

*/

$* 1$

*)

${ }^{*} \mathrm{c}$ ress 


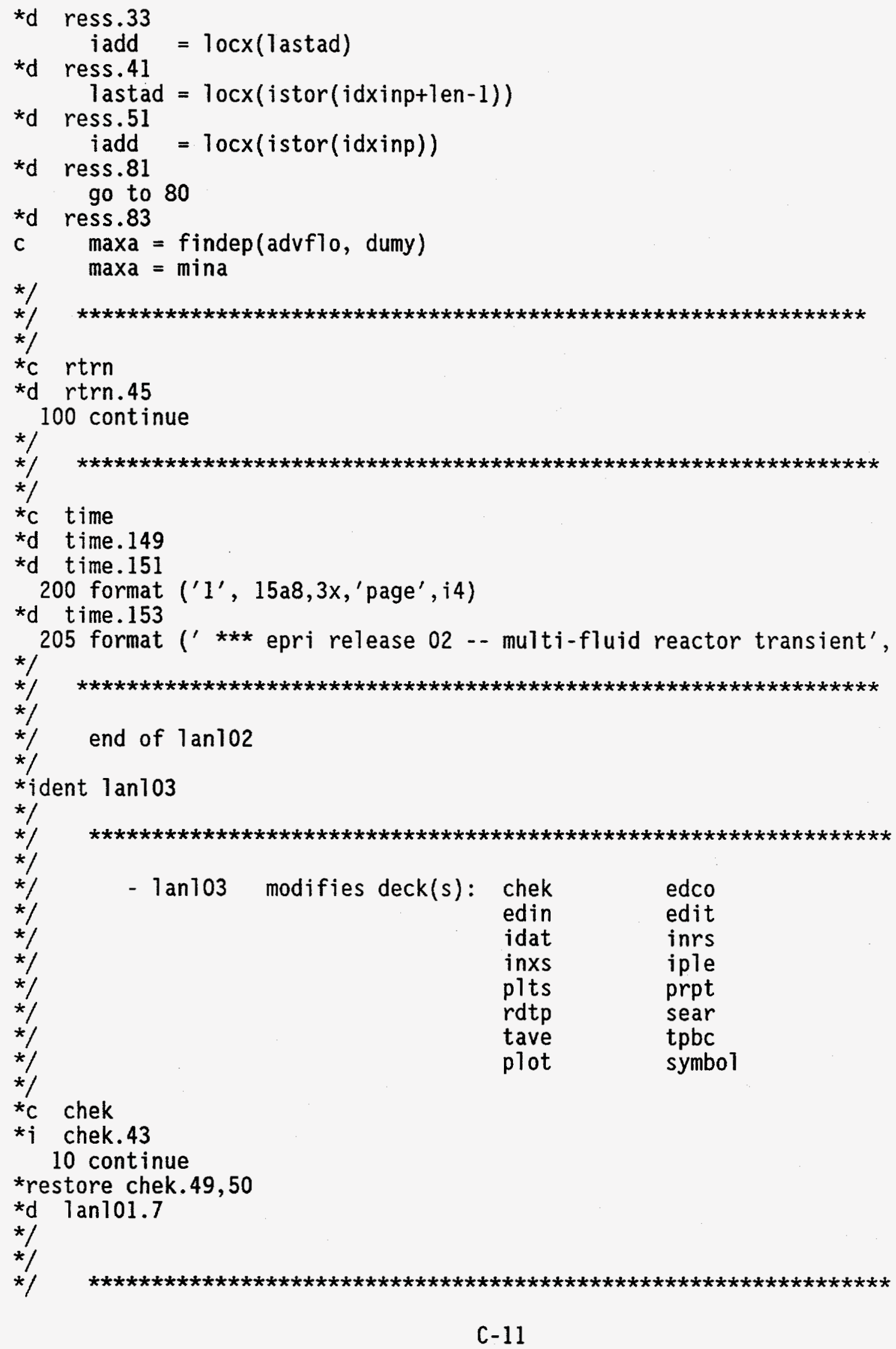




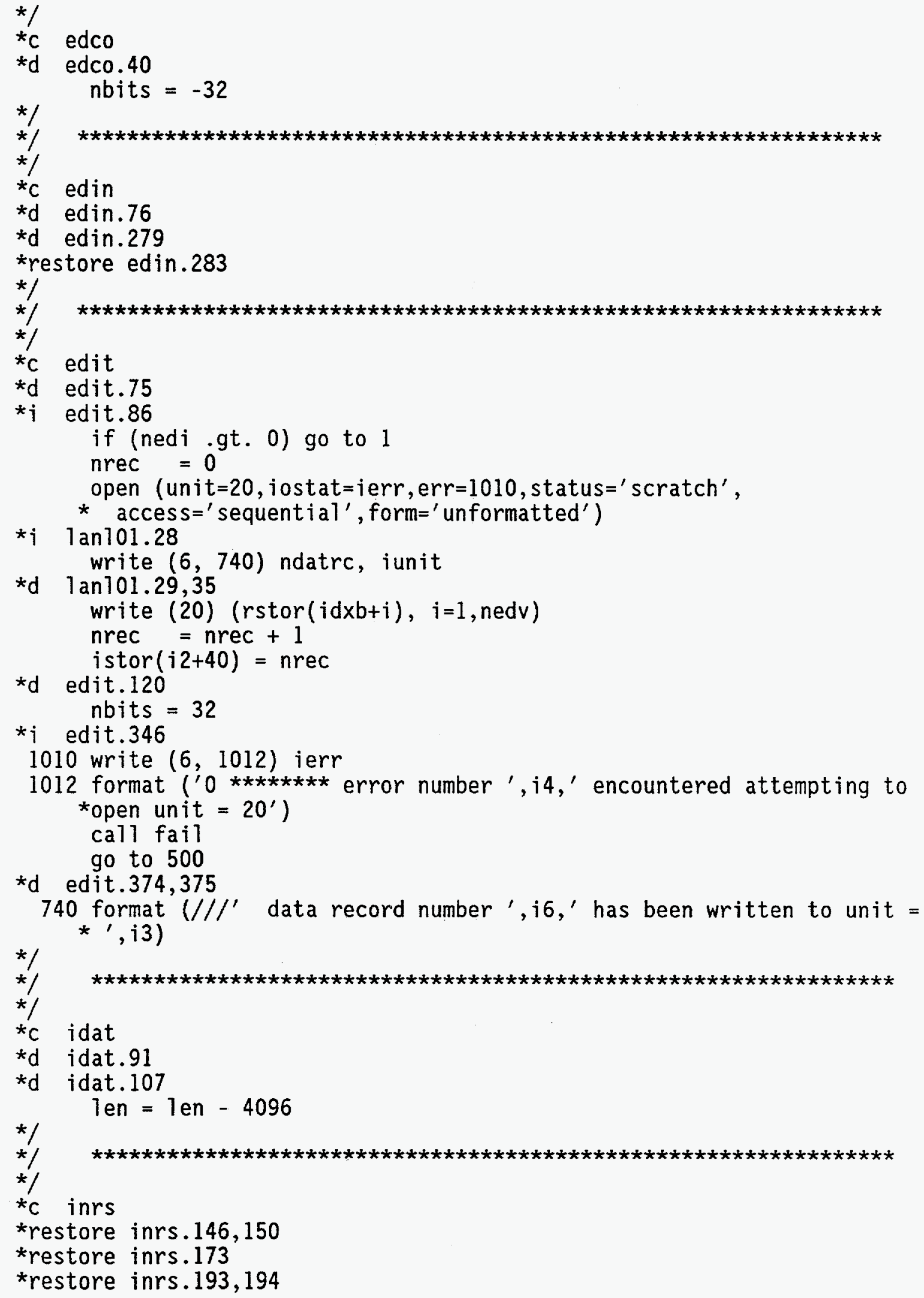


*restore inrs.232,233

*1

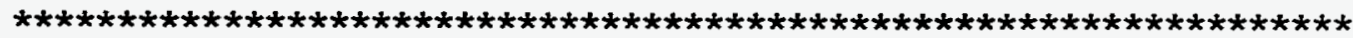

*/

${ }^{*} \mathrm{c}$ inxs

*i inxs.138

open (unit $=40$, iostat $=$ ierr, err $=1010$, status $=^{\prime} 01 d^{\prime}$, file $={ }^{\prime}$ tape $40^{\prime}$,

* access $=$ ' sequential', form='formatted')

$* i$ inxs. 320

1010 write $(6,1012)$ ierr

1012 format ('0******** error number ', $i 4$,' encountered attempting to *open file $=$ tape40') call fail return

$\begin{array}{ll}* & \\ * & * \\ * & \\ * & \end{array}$

*restore iple.166 jus $=3$

*restore iple.195

$\star /$

$\star /$

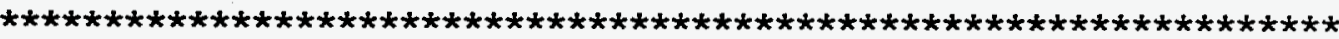

$* /$

${ }^{*} \mathrm{c}$ plts

$*_{i}$ plts.51

tfid $=$ bufy +1.0

*d plts. 57

*d plts.63

nbyte $=8$

*d p plts.65

nbits $=32$

* pits.171

c $\quad$ kset $=$ dataset number of curve

*d pits.178,180

ireel $=1$

*d plts.184

fid $=$ tfid + float (kset) -1.0

*d plts.185,186

iunit $=$ kset +20

*d plts.191

rewind junit

* $j$ plts.193

call reserv (fid, jsiz, 2, idxp)

read (iunit) (rstor (idxp+m-1), $m=1, j$ siz)

*d plts.238

rewind iunit

call delete (fid)

*d plts.323

read (iunit) (rstor (idxp+m-1), $m=1, j$ siz)

index $=\mathrm{idxp}$

*d pits.422 


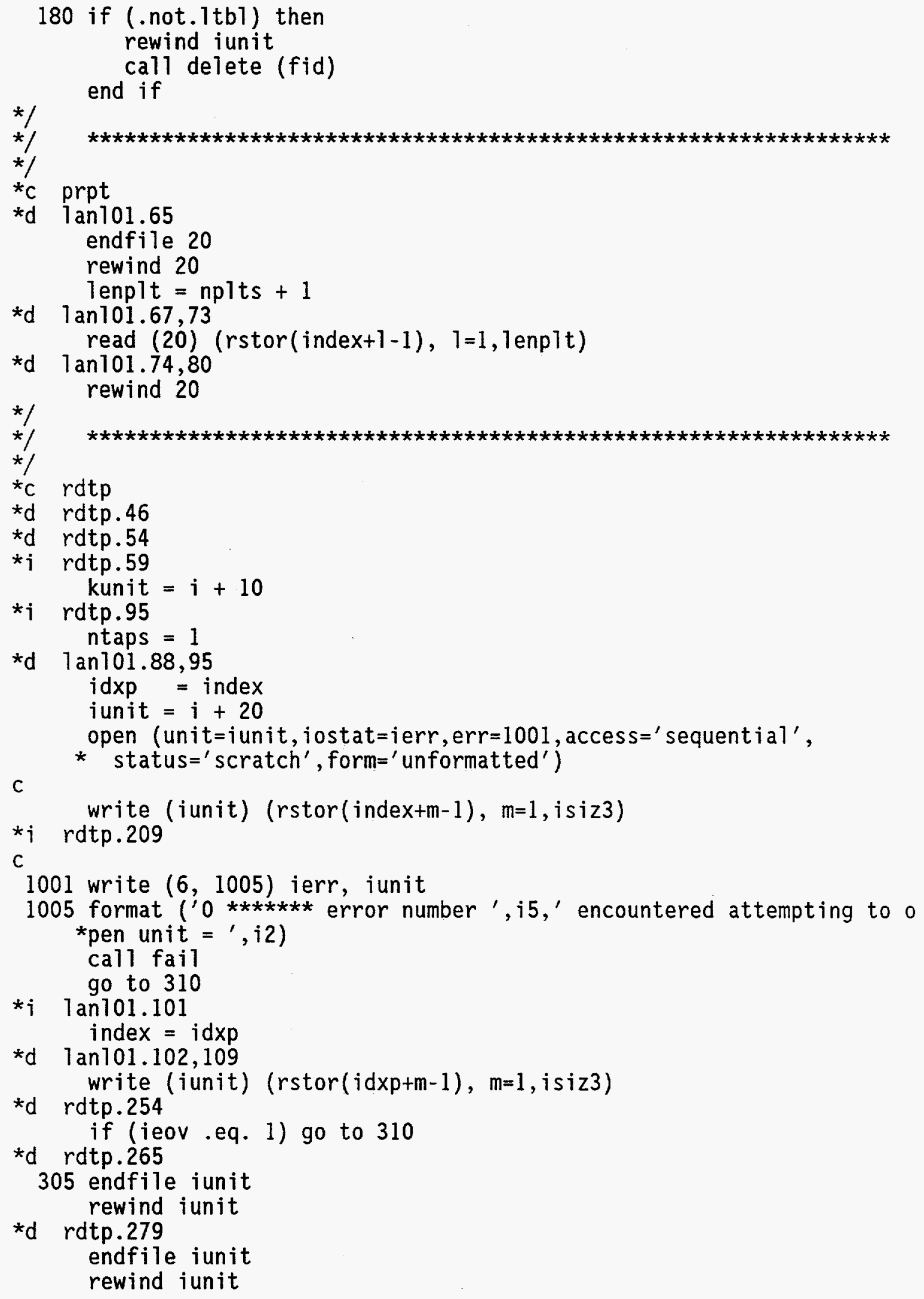




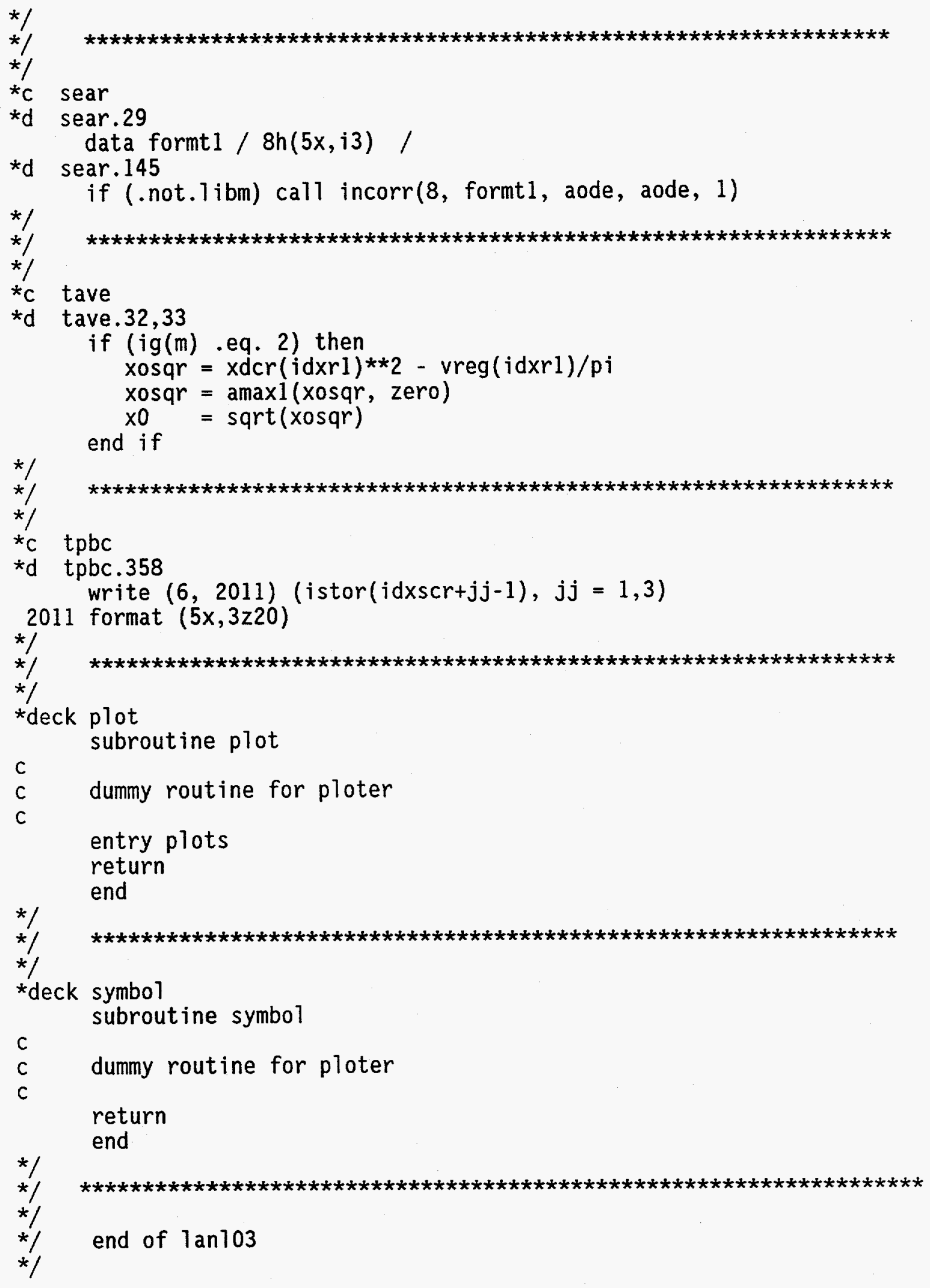

